\title{
NOTAS HISTÓRICAS E ÍNDICES GENERALES (1943-1994) DE LA REVISTA ZOOLÓGICA ESPAÑOLA "GRAELLSIA"
}

\author{
B. Sanchiz (*) y C. Martín (*)
}

\begin{abstract}
RESUMEN
Se presentan, junto a una breve introducción histórica, los índices temáticos de los primeros 50 volúmenes de la revista zoológica española "Graellsia", correspondientes al periodo 1943-1994. Los índices incluyen una relación exhaustiva de los autores, géneros, familias, nuevos taxones, provincias (españolas y portuguesas), islas y países mencionados en los 821 artículos publicados. Los más de 6.800 géneros indexados, incluyendo sinónimos, abarcan una parte considerable de la fauna española conocida.
\end{abstract}

Palabras clave: Zoología, Taxonomía, Faunística, Historia de la Ciencia, Índices.

\section{ABSTRACT \\ Historical notes and general index (1943-1994) of the Spanish zoological journal "Graellsia"}

A brief historical introduction of the Spanish zoological journal "Graellsia", and the general thematic indexes for its first 50 volumes (1943-1994) are presented. The indexes that have been compiled include exhaustive lists of the authors, genera, families, new taxa, Spanish and Portugese provinces, islands, and political countries, as mentioned in the 821 published articles. More than 6.800 genera, including synonyms, have been indexed, and represent a large fraction of the known Spanish fauna.

Key words: Zoology, Taxonomy, Faunistics, History of Science, Indexes.

\section{Introducción}

La revista "Graellsia" es una de las contadas revistas zoológicas españolas publicadas desde la inmediata postguerra. La revista ha reflejado en sus primeros 50 volúmenes los profundos cambios acaecidos en la comunidad científica de nuestro país, tanto respecto al incremento del número de investigadores (cuantificado en Martín Albaladejo, 1994), como en lo concerniente a los métodos, objetivos y técnicas aplicadas, acumulando así una ingente información que resulta ahora de difícil acceso. Parece por ello de interés dar a conocer, mediante índices exhaustivos, los muchos datos de índole esencialmente taxonómica y faunística que se encuentran en sus páginas, y que representan una fracción relativamente importante de la información zoológica acumulada en este periodo 19431994 en España.

Un estudio histórico detallado de la revista Graellsia no resulta aún posible, pues la documentación del desaparecido Instituto Español de Entomología, que fue su editor durante gran parte de su existencia, no se encuentra aún plenamente disponible, estando actualmente en proceso de catalogación e informatización en el archivo del Museo

* Museo Nacional de Ciencias Naturales. José Gutiérrez Abascal, 2. 28006 Madrid. Correo electrónico: mcnb105@fresno.csic.es 
Nacional de Ciencias Naturales. La breve introducción aquí realizada tiene como objetivo el situar en un contexto histórico las cambiantes fases por las que ha pasado la revista, que a lo largo de los años ha ido variando tanto su ámbito taxonómico como sus objetivos.

\section{Métodos}

Se realizó en primer lugar un índice base, numerado secuencialmente, con todos los artículos y notas publicados, un total de 821 , que se relacionan más adelante como Índice General. Todos estos trabajos fueron leídos entre cinco personas, quienes extractaron en fichas todos los términos a incluir en el índice. Posteriormente los autores leyeron todos los trabajos, corrigiendo errores y homogeneizando la información de los lectores iniciales, para pasar después ésta a la base de datos bibliográfica Procite 3 , desde la que se procesó la información y se confeccionaron los índices cruzados.

La fiabilidad de estos índices fue estimada mediante muestreo aleatorio estratificado, utilizando el índice de géneros, que se pretende sea exhaustivo. De 393 nombres de géneros localizados directamente en la revista, sólo ocho no aparecieron correctamente indexados, lo que supone exactitud aproximada del $98 \%$.

\section{Desarrollo histórico}

1943-1964: “GRAELLSIA. REVISTA DE ENTOMÓLOGOS ESPAÑOLES”. INSTITUTO ESPAÑOL DE ENTOMOLOGÍA

La revista se funda en 1943, a iniciativa del Instituto Español de Entomología, un centro entonces de reciente creación perteneciente al Consejo Superior de Investigaciones Científicas (CSIC). Debe entenderse que esa institución se subrroga en la edición de la prestigiosa revista Eos, posiblemente la mejor revista entomológica en lengua castellana, fundada ya en 1925 y publicada hasta entonces por el Museo Nacional de Ciencias Naturales, entidad de la que se segrega el Instituto Español de Entomología en 1941. Los fundadores de Graellsia fueron Ramón Agenjo Cecilia, Gonzalo Ceballos Fernández de Córdoba y Eduardo Zarco Segalerva, fide Agenjo [artículo 383]. La intención y justificación original fue iniciar una publicación periódica "Consagrada fundamentalmente .... a aquellos aficionados a la
Entomología que carecen tanto de medios bibliográficos como de relación que les permita dar a la publicidad sus estudios y observaciones...", y su ámbito temático el "versar sobre temas de biología, sistemática, ecología o cualquier otra rama de las Ciencias Naturales relacionadas con la clase de los insectos", según advierte la contraportada de los primeros volúmenes y elabora prolijamente la propia presentación de la revista [artículo 001]. Se publicó por ello inicialmente en formato bimensual con 6 números anuales. Esta dedicación al colectivo de los entomólogos no profesionales no debe entenderse como meramente "divulgadora", en su acepción lúdica actual de servir como distracción al lector, sino desde un planteamiento científico. En un contexto social anterior a la introducción de la fotocopia y con grandes dificultades para obtener bibliografía, por la pobreza y aislamiento español y la guerra mundial, se pretendía facilitar su labor al estudioso local y establecer algún tipo de red informal de intercambio de datos faunísticos entre entomólogos no profesionales, que pudieran servir para la elaboración futura de faunas nacionales. De hecho, el Instituto Español de Entomología ofertaba incluso la identificación de los ejemplares que le fueran remitidos.

Los dos pivotes fundacionales de Graellsia, ser una revista entomológica y estar orientada básicamente a principiantes, inmediatamente se adaptaron a la realidad existente. Entomología se interpretó de una manera laxa, pues ya desde el primer volumen se publicaron artículos sobre artrópodos no insectos. También desde el principio sus páginas se fueron llenando de trabajos de científicos profesionales, y resulta sintomático, por ejemplo, que el primer artículo de la nueva revista (después de una obligada biografía de M.P. Graells, origen del nombre de la cabecera editorial) se deba a F. Español, un entomólogo profesional, y se describa allí una especie nueva para la ciencia.

El colectivo de entomólogos de la época, un reducidísimo número de personas interesadas en los insectos, la orientación académica, y el coste económico, ciertamente no justificaba una publicación con distribución bimensual, y aconsejaron desde el volumen 6 pasar a una periodicidad generalmente anual, aunque ocasionalmente fue semestral. Durante toda esta etapa coexisten artículos de orientación muy académica con otros que utilizan un lenguaje más coloquial, e incluso ocasionalmente se publican artículos no inéditos. Sin embargo, es precisamente al final de la etapa cuando se incluyen verdaderos artículos de divulgación, como algunos 
curiosos guiones radiofónicos debidos a I. Docavo. Los artículos eran juzgados por un Comité de Redacción en el que intervenía la mayoría de la plantilla científica del Instituto Español de Entomología.

1965-1977: "GRAELLSIA. REVISTA DE ENTOMÓLOGOS IBÉRICOS”. INSTITUTO ESPAÑOL DE ENTOMOLOGÍA

A partir del tomo 21 (1965) la revista cambia de subtítulo y se convierte en "Revista de entomólogos ibéricos", acojiendo trabajos sobre la entomofauna peninsular que traten de "cuanto a ella se dedique y esté redactado en nuestras dos excepcionales lenguas". Se publican así estudios de colegas portugueses, en castellano o portugués, y se aceptarían en catalán [artículo 522]. Esta etapa está caracterizada por la dirección de R. Agenjo Cecilia y la secretaría ejecutiva de D. Selga Serra. Son curiosas las estériles polémicas surgidas entre lepidopterólogos acerca de prioridades taxonómicas a dilucidar en función de las fechas de publicación de Graellsia y Shilap, iniciada esta última en 1973. Temáticamente se publican sobre todo trabajos faunísticos, y en menor medida de índole aplicada, experimental y taxonómica. El tomo 25 incluye una historia de la génesis de Graellsia, y una lista de subscriptores, publicándose también casi un centenar de páginas con cartas de apoyo a la continuidad, entonces amenazada, de la revista.

1978-1984: “GRAELLSIA. REVISTA DE ENTOMÓLOGOS IBÉRICOS”. INSTITUTO ESPAÑOL DE ENTOMOLOGÍA

Los volúmenes 34 a 40 tienen como director a S.V. Peris Torres, siendo secretarios ejecutivos A. Compte Sart primero y M.P. Martín Mateo después. Los objetivos se hacen de nuevo explícitos: "La revista ... tiene por objeto la publicación de trabajos sobre morfología, fisiología y ecología de los artrópodos, así como sistemática preferentemente dedicada a la Península Ibérica. Complementariamente, atiende a la puesta al día de temas especiales entomológicos, lo mismo que a noticias y recensiones de publicaciones de interés para los investigadores españoles" [Contraportada]. Según testimonios coincidentes de personas integrantes del Consejo de Redacción de entonces, los manuscritos que se recibían para su publicación en Eos o Graellsia eran relativamente similares en calidad, y se prefería adjudicar a Graellsia los temas más experimentales, aplica- dos, fenológicos etc, así como faunística, reservando los artículos de índole más sistemática y taxonómica para Eos. Por esta causa, Graellsia adquiere un cierto protagonismo durante esta etapa en temas de Entomología aplicada, una vertiente que desaparece a partir de 1985. En esta época Graellsia es pionera en España en aplicar a la Entomología algunos instrumentos que luego se han desarrollado extraordinariamente, como pueden ser por ejemplo el uso de electroforesis [artículo 594], estadística por ordenador [604], análisis de distribución UTM [614], dendrogramas fenéticos [613], etc.

1985-1987: “GRAELLSIA. REVISTA DE ENTOMÓLOGOS iBÉRICOS". Museo Nacional de Ciencias NATURALES

La reunificación del Museo Nacional de Ciencias Naturales, con la incorporación al mismo del Instituto Español de Entomología, hace que Graellsia pase a ser publicada por el departamento de Entomología del Museo. Este cambio administrativo conlleva pocas variaciones reales en la temática de la revista, que tiene en esta etapa a M.P. Martín Mateo y E. Plaza Infante como editoras científicas ejecutivas, y la dirección nominal, entonces preceptiva, de los sucesivos directores del Museo, E. Aguirre Enríquez y C. Sáenz Laín.

1988-1993: "Graellsia. Revista de Zoología". Museo Nacional de Ciencias Naturales

El Museo Nacional de Ciencias Naturales carecía en 1988 de revista zoológica, publicando en cambio dos revistas entomológicas. Por ello, reservando Eos para Entomología, se decide cambiar el ámbito temático de Graellsia a partir del volumen 44, pasando a publicar "... trabajos de investigación original, preferentemente de tipo monográfico o que presenten un tratamiento en profundidad de un tema en cualquier área de la Zoología básica" [artículo 696]. Ejercen de editores científicos ejecutivos J.L. Nieves Aldrey, M.A. Ramos Sánchez y A. Salvador Milla, junto con un nutrido Comité Editorial formado en su totalidad por científicos no pertenecientes al Museo. El formato de la revista pasa a ser de dos columnas, y los manuscritos se revisan ya mediante doble evaluación externa. Aunque la temática abarca cualquier tema zoológico, y continúa publicándose activamente Eos, Graellsia mantiene una alta proporción de artículos sobre Entomología. 
1994 HASTA LA ACTUALIDAD: "GRAELLSIA". Sociedad DE AMigos Del Museo Nacional DE CiEnCIAS NATURAles

Un editorial del volumen 49 [artículo 774] explica clara y suscintamente las razones para un drástico cambio en Graellsia: “... El volumen 49 que tienen en sus manos, correspondiente a 1993, supone sin embargo el obligado fin de una etapa, ya que la Junta de Gobierno del C.S.I.C., siendo presidente del organismo el Dr. José $M^{a}$ Mato de la Paz, acordó en su reunión de 30 de octubre de 1992 suprimir a partir de primero de enero de 1993 la subvención que el C.S.I.C. destinaba a su edición. Esta decisión fue reprobada testimonialmente por el Claustro Científico del Museo Nacional de Ciencias Naturales con un único voto en contra. Tanto el notorio incremento de calidad científica que se venía observando en la revista, como el enriquecimiento que para las bibliotecas del C.S.I.C. suponían las 161 publicaciones (139 extranjeras) con las que se intercambiaba, no aconsejaban en opinión de los científicos del Museo su supresión. ..... El Departamento de Biodiversidad del Museo .... acordó continuar la edición de Graellsia en régimen de autofinanciación, restringiéndola temáticamente a estudios relacionados con la Biodiversidad". En esta nueva etapa la revista publica trabajos científicos sobre "Biodiversidad zoológica, incluyendo estudios taxonómicos, faunísticos, biogeográficos, corológicos, evolutivos y de conservación, con preferencia por el ámbito geográfico hispano". La cancelación de bastantes revistas del C.S.I.C., decidida entonces por los rectores del organismo aplicando criterios selectivos poco convincentes, desgraciadamente afecta también a Eos, que se venía publicando ininterrumpidamente desde 1925, incluyendo cada año de la Guerra Civil y la postguerra. A partir del volumen 50 la cabecera de Graellsia pasa a la Sociedad de Amigos del Museo Nacional de Ciencias Naturales, quien se encarga desde enton- ces de su edición en régimen de autofinanciación. El brusco cambio de este proceso, y la necesidad de autofinanciarse y a la vez mantener sus intercambios con otras revistas, provoca inevitables retrasos en la fecha de publicación, que finalmente pueden regularizarse, habiendo aparecido hasta la fecha 53 volúmenes. Actúan como editoras ejecutivas en esta crítica etapa A.I. Camacho Pérez y M.A. Ramos Sánchez.

\section{AGRADECIMIENTOS}

Agradecemos muy especialmente la eficaz colaboración de Jorge M. Lobo, Mercedes París, Arabia Sánchez Terrón y Celia Santos en la lectura inicial de los artículos, extractando su información en fichas. El personal y Departamento de Biodiversidad y Biología Evolutiva del Museo Nacional de Ciencias Naturales (CSIC), y en especial la Dra. Ana I. Camacho, jefa del Departamento durante el periodo de elaboración del trabajo, impulsaron y patrocinaron esta iniciativa de muy distintas maneras, y ciertamente el trabajo no hubiera podido finalizarse sin su ayuda.

\section{Referencias}

Martín Albaladejo, C. 1994. Bibliografía entomológica de autores españoles (1758-1990). In: M. Ramos (ed.), Documentos Fauna Ibérica 1. Museo Nacional de Ciencias Naturales, CSIC, Madrid. 823 págs.

Publicado, el 30-VI-1998 


\section{ÍNDICE DEL CONSEJO DE REDACCIÓN Y OTRO PERSONAL EDITORIAL}

A partir del volumen 23 ya figuran explícitamente los nombres de las personas involucradas en la confección de la revista, que aquí relacionamos junto con las funciones que realizaban y los volúmenes en que participaron. Esta información procede de las propias publicaciones, y no ha sido confrontada con la que pudiera existir en el archivo del Museo Nacional de Ciencias Naturales. La lista de evaluadores se viene publicando sólo desde el volumen 50.

Abreviaturas: C: Colaborador asesor. CR: Consejo de Redacción. D: Director. DP: Diseño de portada. S: Secretaría General (Editor científico). DA: Directora científica adjunta. SR: Secretaría de Redacción. E: Evaluador. Se indican entre paréntesis los volúmenes en que se participa.

Agenjo Cecilia, C.: C(23-33)

Agenjo Cecilia, R.: D (23-33)

Aguirre Enríquez, E.: D(41)

Alba Tercedor, J.: E(50)

Alfaro Arregui, P.: C(23-24)

Almeida Fernandes, J. de: CR(23-33)

Alvarez Sánchez, J.: C(23-33), CR(34-41)

Anduiza, R.: C(23-33)

Arias Delgado, M.: CR(34-40, 44-49)

Arozarena Doblado, R.: C(23-33)

Arroyo Varela, M.: C(23-33)

Azevedo Silva, F.: C(23-33)

Bach Piella, C.: CR(42-43)

Baeta Neves, C.M.: CR(23-33, 37-41)

Báguena Corella, L.: C(23-32)

Balcells Rocamora, E.: C(23-33)

Barrientos Alfageme, J.A.: CR(43)

Bellés Ros, X.: CR(44-49)

Bello Rojo, E.: E(50)

Benítez Morera, A.: C(23-26)

Bonnet, P.: C(27-33)

Bullón Ramírez, A.: C(23-32)

Cadahía Cicuéndez, D.: C(23-33)

Camacho Pérez, A.I.: S(50)

Cañizo Gómez, J. del: C(23-26)

Capilla Caballero, J.: C(23-32)

Cardoso Cabral, M.T.E.: C(23-33)

Carmona, M.M.: C(23-33)

Carrascal de la Puente, L.M. : E(50)

Castro Guillermín, L. de: C(23-33)

Ceballos Jiménez, P.: C(23-33)

Cei, J.M.: E(50)

Cobos Sánchez, A.: C(23-33)

Coca Abia, M.: SR(50)

Codina Padilla, F.: C(23-33)

Compte Sart, A.: C(23-33), S(34-37), CR(38-43)

Dicenta Ballester, A.: C(23-33)

Diniz, M. de Assunção: CR(23-33)

Docavo Alberti, I.: CR(23-33)

Espadaler Gelabert, X. : E(50)

Español Coll, F.: CR(23-33)

Esteban, L.: DP(1-43)

Feliú, F.: C(23-33)

Fernández, J.Mª : C(23-33)

Fernández López, J.: SR(50)

Fernández Porter, C.: C(23-33)

Flores Casas, H.: C(23-33)

Gamo García, J. : E(50)

García del Jalón, D.: CR(44-49)

García de Viedma, M.: C(23-26)

García París, M.: E(50)
García-Valdecasas Huelin, A.: CR(50), E(50)

Gómez Aizpurúa, C.: C(23-26)

Gómez Agüero, L.M.: SR(46-50)

Gómez-Menor Guerrero, J.M.: C(23-33)

Gómez-Menor Ortega, J.: CR(23-33)

Gómez Ruano, R.: C(23-32)

González López, F.: C(23-33)

Gosálbez Noguera, J.: CR(44-49)

Haro Vera, A. de: CR(23-33)

Ibáñez Genis, M. : E(50)

Ibarra Montis, M. de: C(23-33)

Isart Sabí, J.: C(23-33)

Jiménez Millán, F.: C(23-33)

Jordana Butticaz, R. : E(50)

Lagar Mascaró, A.: C(23-33)

Llopis Mínguez, B.: C(23-33)

Llorente del Moral, V.: C(23-33), CR(34-43), E(50)

López de la Cuadra, C.: E(50)

Manga González, Y.: E(50)

Marcos Gilaranz, I.: SR(31-33)

Marcos García, M.A.: E(50)

Margalef López , R.: C(23-33)

Martín Cano, J.: CR(44-49)

Martín Mateo, M.P.: C(23-33), CR(34-37), S(38-42), DA(43)

Martínez Ansemil, E.: E(50)

Mateu Sanpere, J.: C(23-33)

Michelena Saval, J.M.: E(50)

Mingo Pérez, E.: C(23-33), CR(34-43)

Monserrat Montoya, V.: CR(44-49)

Montada Brunet, J.: C(23-33)

Montero Cosano, M.: C(23-33)

Morales Agacino, E.: C(23-26)

Morillo Fernández, C.: CR(34-36)

Nájera Angulo, L.: C(23-32)

Nascimento Ferreira, R. do: C(23-33)

Nieves Aldrey, J.L.: S(44-49), CR(50), E(50)

Orejón, J.M.: C(23-25)

Palau Camps, J.M ${ }^{\mathrm{a}}$. C(23-33)

Pardo Alcaide, A.: C(23-32)

Pérez-Íñigo Quintana, C.: C(25-33), E(50)

Pérez Mellado, V.: CR(44-49)

Pérez Zaballos, J.M.: E(50)

Peris Torres, S.V.: CR(23-33, 42-43), D(34-40)

Plaza Infante, E.: DA(42), S(43)

Portillo Rubio, M.: E(50)

Prieto Sierra, C.: E(50)

Presa Asensio, J.J.: CR(42-43)

Pujade Villar, J.: E(50)

Purroy, F.J.: CR(44-49)

Ramos Sánchez, M.A.: S(44-50) 
Rey Arnáiz, J.Ma.: CR(34-40)

Robles Chillida, E.M.: C(25-32)

Rodríguez Alfaro, M $^{\mathrm{a}}$.C.: SR(23-33, 42-43)

Ruaix, A.: C(23-32)

Rupérez Cuellar, A.: CR(23-33)

Sáenz Laín, C.: D(42-43)

Sala de Castellarnau, I.: C(23-32)

Salvador Milla, A.: S(44-48)

Sanchiz Gil de Avalle, F.B.: CR(50), E(50)

Santoro, F.H.: C(23-32)

Selga Serra, D.: S(23-33), CR(42-43)

Serrano, J.M.: E(50)

Serrano Marino, J.: CR(44-49), E(50)
Serrão Nogueira, C.D.: C(23-33)

Subías Esteban, L.: CR(42-43), E(50)

Sousa Alvim, H. de: C(23-33)

Suárez Egea, F.J.: C(23-33)

Templado Castaño, J.: CR(23-42-43)

Templado González, J.: CR(50), E(50)

Uriz Lespe, M.J.: CR(44-49)

Valle de Lersundi, M.: SR(31-33)

Varea de Luque, A.: C(23-32)

Vergés Serra, F.: C(23-33)

Vieitez Martín, J.M.: CR(44-49)

Weidner Lucas, I.: SR(23-30)

Xostoa, A.: CR(44-49) 


\section{ÍNDICE GENERAL}

Se utilizan los nombres de autores y títulos tal cual fueron publicados. La numeración asignada a los artículos es la referencia utilizada en los índices temáticos de Autores, Nuevos taxones, Géneros, Familias, Geográfico, y Países e Islas.

001. Ceballos Fernández de Córdoba, Gonzalo. 1943. [Presentación]. 01(1): 003-006

002. Agenjo, R. 1943. Biografía de don Mariano de la Paz Graells Agüera. 01(1): 007-021.

003. Español Coll, Francisco. 1943. Datos para el conocimiento de los tenebriónidos del Mediterráneo occidental. 01(1): 023-028.

004. Nájera Angulo, L. 1943. Los aedinos españoles y el peligro de la Fiebre Amarilla. 01(1): 029-035.

005. Anónimo. 1943. Noticiario entomológico. $\mathrm{V}^{\mathrm{a}}$ Entrega de la "Faune Entomologique de l'Andalousie" (Lepidópteros). La exploración del Pirineo. Envío de material para recolección. Ha fallecido el Dr. Menozzi. Un posible foco de "fiebre de papataci" en España. Los médicos entomólogos. Faune de France. Vol. 38: "Coléoptères Scarabéides" par R. Paul Lechevalier et Fils. 12, Rue de Tournon (Vie). Paris 1941. La Biblioteca de la Sociedad Entomológica Italiana. 01(1): 037-039.

006. Cañizo, José del. 1943. Notas sobre la "palomilla gris" de la harina ("Ephestia kühniella" Zeller). 01(2): 003-009.

007. Palau, José $\mathrm{M}^{\mathrm{a}}$. 1943. Captura del "Cylindropsis balearica" Breit en el predio "Biniatzar" de Buñola (Mallorca) (Col. Staphylinidae). 01(2): 011-012.

008. Pujol, M. 1943. Catálogo de lepidópteros que se encuentran en la zona norte de los alrededores de Madrid. 01(2): 013-028, lámina III

009. Anónimo. 1943. Noticiario entomológico. La Medalla Fabricius". El Dr. Jakob Daublebsky von Sterneck. El Dr. Adolfo Lutz. "Fauna entomológica de la vid". La Dorífora en España. 01(2): 029032.

010. Alvarez, J. 1943. Ideas fundamentales para la cría de insectos en cautividad. 01(3): 003-006.

011. Margalef, Ramón. 1943. Sobre la ecología de las larvas de algunos culícidos (Dipt. Cul.). 01(3): 007-012.

012. Pujol, M. 1943. Catálogo de lepidópteros que se encuentran en la zona norte de los alrededores de Madrid. (Continuación). 01(3):013-028.

013. Anónimo. 1943. Noticiario entomológico. El Profesor Porter. Nueva edición de la "Entomología y Parasitología Agrícolas" de Génaux. El interés de la formación del catálogo de insectos parásitos en los futuros planes de lucha biológica. 01(3): 029-032.

014. Nájera, L. 1943. Nuevos datos sobre la distribución geográfica de los "Phlebotomus" en España. 01(4): 003-012.

015. Benítez Morera, A. 1943. Los medios de defensa de los insectos. 01(4): 013-015.

016. Pujol, M. 1943. Catálogo de Lepidópteros que se encuentran en la zona norte de los alrededores de Madrid. (Continuación). 01(4): 017-030.
017. Anónimo. 1943. Noticiario entomológico. El Prof. Paul Marchal. La importancia de las niguas de los ejércitos. Las "tsé-tsé" africanas. "Phlebotomus" del Congo belga. Culícidos españoles. 01(4): 031-032.

018. Ceballos, G. 1943. Las hormigas. 01(5): 003-008.

019. Pujol, M. 1943. Catálogo de Lepidópteros que se encuentran en la zona norte de los alrededores de Madrid. (Conclusión). 01(5): 9-29.

020. Anónimo. 1943. Noticiario entomológico. Una mariposa emigrante: "Celerio lineata livornica" Esp. 01(5): 031-032.

021. Nájera, L. 1943. Notas históricas sobre Entomología médica. I. El ácaro productor de la sarna. 01(6): 003-011.

022. Vidal López, Manuel. 1943. Catálogo provisional de neurópteros de la región levantina. 01(6): 013024.

023. Ibarra, Mariana de. 1943. Nota necrológica. Ignacio de Sagarra y de Castellarnau (1889-1939). 01(6): 025-032.

024. Margalef López, Ramón. 1944. Notas sobre quironómidos. I (Ins. Dipt.). 02 (1): 003-013.

025. Zariquiey Alvarez, Ricardo. 1944. Contribución al conocimiento de la distribución de los "Phlebotomus" en España. 02(1): 015-020.

026. Alvarez Sánchez, J. 1944. Curiosidades de las arañas. 02(1): 021-025.

027. Español Coll, Francisco. 1944. Nota necrológica. Ricardo Zariquiey Cenarro (1870-1943). 02(1): 027-031.

028. Pardo Alcaide, Anselmo. 1944. Analectas entomológicas. I. Las especies raras: su concepto, localización y captura. 02(1): 033-041.

029. Varea de Luque, A. 1944. En busca de la "Synchloë callidice" (Esper) por el Pirineo de Lérida. Impresiones de caza. 02(2): 043-051.

030. Agenjo, R. 1944. Nota necrológica. R. P. Candido Mendes D’Azevedo, S.J. (1874-1943). 02(2): 053-059.

031. Anónimo. 1944. Noticiario entomológico. La Real Sociedad Entomológica de Egipto. Los insectos en la literatura (de la obra "Recuerdos de niñez y de mocedad", de don Miguel de Unamuno). 02(2): 061-064.

032. Margalef López, Ramón. 1944. Notas sobre quironómidos. II (Ins. Dipt.). 02(3): 065-076.

033. Sala de Castellarnau, Ignacio. 1944. La invernación en los insectos. 02(3): 077-087, lámina I.

034. Orejón, J. M. 1944. La influencia de los rayos ultravioleta sobre los insectos. 02(3): 089-095.

035. Feliu Quadreny, Sebastián 1944. Leyendas y tradiciones sobre algunos insectos en Mallorca. 02(3): 097-099.

036. Español Coll, Francisco. 1944. Datos para el conocimiento de los tenebriónidos del Mediterráneo occidental. 02(4): 101-108. 
037. Varea de Luque, A. 1944. Una corta campaña lepidopterológica en Uña (Cuenca). 02(4): 109-117.

038. Fernández Porter, Carlos. 1944. Introducción al catálogo de los carábidos valencianos. 02(4): 119132.

039. Ceballos, Gonzalo. 1944. Las hormigas II. 02(5): 133-140.

040. Pardo Alcaide, Anselmo. 1944. Analectas entomológicas II. Las cajas de colección (normas para su construcción). 02(5): 141-154.

041. Benítez Morera, Antonio. 1944. Los medios de defensa de los insectos. 02(5): 155-163.

042. Margalef López, Ramón. 1944. Notas sobre quironómidos (Ins. Dipt.). III 02(6): 165-181.

043. Pardo Alcaide, Anselmo. 1944. Analectas entomológicas III. Las cajas de colección (normas para su construcción). 02(6): 183-188.

044. Anónimo. 1944. El nuevo insecticida DDT. 02(6): 189-191.

045. Sala de Castellarnau, Ignacio. 1945. La invernación en los insectos II. Lepidópteros. 03(1): 003011.

046. Margalef López, Ramón. 1945. Notas sobre quironómidos. IV (Ins. Dipt.). 03(1): 013-022.

047. Palau, José María. 1945. Notas para un catálogo de los Coleópteros de Mallorca. I. Fam. Tenebrionidae. 03(1): 023-036.

048. Lagar Mascaró, Angel. 1945. Observaciones del "Meladema coriaceum" en España y Marruecos. 03(1): 037-038.

049. Anónimo. 1945. Noticiario entomológico. Poco dura la alegría en casa del pobre (contra el DDT). Las ondas dirigidas. Una antigua y pintoresca cuestión entomológica tratada en serio. 03(1): 039-040.

050. Ceballos, G. 1945. Gente conocida. I. Las pulgas. 03(2): 041-048.

051. Varea de Luque, A. 1945. Dialogo entre Parnassius. 03(2): 049-053.

052. Docavo Alberti, Ignacio. 1945. Mis principios entomológicos. 03(2): 055-061.

053. Pardo Alcaide, Anselmo. 1945. Analectas entomológicas. IV. Los Brachycerus Ol. hispano-marroquíes. 03(2): 063-070.

054. Cañizo, José del. 1945. Investigaciones de Cajal sobre los ojos y las fibras musculares de algunos insectos. 03(3): 073-089.

055. Agenjo, Ramón. 1945. Sobre la inobservancia de algunas reglas y principios de nomenclatura. 03(3): 091-096.

056. Varea de Luque, A. 1945. Algunas mariposas viajeras. 03(3): 097-108

057. Ceballos, Gonzalo. 1945. Gente conocida. II. Los piojos. 03(4): 109-117.

058. Saz, Eugenio 1945. La puesta de huevos de Zonitis immaculata O1. 03(4): 119-123.

059. Benítez Morera, Antonio. 1945. Los medios de defensa de los insectos. III. 03(4): 124-127, lámina I.

060. Cañizo, José del. 1945. Un notable entomólogo español incógnito: D.P.R.N. 03(4): 129-132.
061. Flores, H. 1945. Contribución al conocimiento de los lepidópteros madrileños. Fáunula de la villa de Alcobendas 03(4): 133-153.

062. Mateu, Joaquín. 1945. Resultados de una excursión entomológica a la Sierra del Aralar (Col. Carábidos). 03(5): 155-164.

063. Palau, José María. 1945. Coleópteros nuevos o interesantes de Mallorca. 03(5): 165-168.

064. Varea de Luque, A. 1945. Una buena localidad para Melitaea aetherie (HB.). 03(5): 169-171.

065. Anónimo. 1945. Noticiario entomológico. Ferdinand Le Cerf. Insectos, hombres y radiaciones ultravioletas 03(5): 173-174.

066. Ceballos, G. 1945. Gente conocida. III. Las chinches. 03(6): 176-183.

067. Español Coll, F. 1945. Nota sobre la captura del Hypogeobium jordai Reitt., pterostíchido endógeo de la fauna balear. 03(6): 185-187.

068. Varea de Luque, A. 1945. Un día a arctíidos en los pinares de Guadarrama. 03(6): 189-197.

069. Anónimo. 1945. Noticiario entomológico. Protección de los cultivos contra los acrídidos por un extracto de Melia. Phthorimacea ocellatella en los remolachares del Alto Ebro. Algunas observaciones sobre polillas de lanas, pieles y plumas. El empleo de la naftalina contra las puestas de Melolontha. 03(6): 199-202.

070. Ceballos, G. 1946. Gente conocida. IV. Las polillas. 04(1): 001-010.

071. Sala de Castellarnau, Ignacio. 1946. La invernación en los insectos. III. Coleópteros. 04(1): 011017.

072. Fernández, José Ma . 1946. Anopheles del subgénero "Myzomyia", de Tenerife. 04(1): 019-023.

073. Benítez Morera, Antonio. 1946. Las orugas. Cómo se crían, preparan y conservan. 04(2): 025036.

074. Varea de Luque, A. 1946. Algunas capturas interesantes. 04(2): 037-046.

075. Español Coll, F. 1946. Don Jose María Mas de Xaxars y Palet (1881-1946). 04(2): 047-052.

076. Agenjo, R. 1946. Recuerdos. Los "Caramelos infantiles". 04(3): 053-066.

077. Lagar Mascaró, Angel. 1946. Introducción al catálogo de ditíscidos, girínidos, hidrofílidos y driópidos de Ceuta y sus alrededores. 04(3): 067-071.

078. Fernández, José María. 1946. Coleópteros viajeros. 04(3): 073-077

079. Castro, Luis de. 1946. Una nueva cita de "Allionix quadrmalatus" Shall (Col. Cler.) en Cataluña. 04(3): 078

080. Agenjo, Ramón. 1946. Catálogo ordenador de los lepidópteros de España. Prólogo. Lista de familias. Micropterigydae y Hepialidae. 04: sin paginar.

081. Sala, Ignacio. 1946. El naturalista D. José Giner Marí. 04(4): 081-087.

082. Español Coll, Francisco. 1946. La Zariquieya troglodytes Jeann. 04(4): 089-097.

083. Castro, Luis de. 1946. Emigración de Orthetrum? (Odonata) (Caballitos del diablo). 04(4): 099-100. 
084. Montero Cosano, Manuel. 1946. Un insecticida contra los pulgones. Los afídidos. 04(4): 101-103.

085. J. del C. 1946. La mariposa de la seda. 04(4): 105107.

086. Agenjo, R. 1946. Catálogo ordenador de los lepidópteros de España. Adelidae, Psychidae, Heterogynidae, Aegeriidae, Thyrididae, Cossidae, Limacodidae y Epipyropidae. 04: sin paginar.

087. Español Coll, F. 1946. Sobre el curioso hábitat de la Adistemia watsoni Woll. (Col. Lathridiidae). 04(5): 109-113.

088. Montada Brunet, Juan. 1946. Coleópteros del macizo de Montseny (Barcelona). 04(5): 115-117.

089. Varea de Luque, A. 1946. Algunos ropalóceros del Pirineo leridano. 04(5): 119-132.

090. Agenjo, Ramón. 1946. Catálogo ordenador de los lepidópteros de España. Thyrididae (ex errore), Anthroceridae, Drepanidae, Cymatophoridae, Axiidae, Notodontidae, Thaumetopoeidae y Syntomidae. 04(5): sin paginar.

091. Flores Casas, H. 1946. Cría "ex-ovo" del Charaxes jasius L. (Lep. Nymph.). 04(6): 133-137.

092. Palau Camps, José María. 1946. Los coleópteros de Torre d'en Pau (Mallorca). 04(6): 139-145.

093. Varea de Luque, A. 1946. Un domingo entre las mariposas de Aranjuez. 04(6): 147-158.

094. Castro, Luis de. 1946. Versión española del prólogo de la obra "Le farfalle diurne d'Italia" de Ruggero Verity, y comentario. 04(6): 159-162.

095. Agenjo, R. 1946. Catálogo ordenador de los lepidópteros de España. Nolidae, Westermanniidae, Arctiidae y Agrotidae (hasta hamifera). 04(6): sin paginar.

096. Mateu Sanpere, Joaquín. 1947. Los carábidos de la Delta del río Llobregat. 05(1): 001-019.

097. Fernández, José María. 1947. Entomología médica. 05(1): 021-030.

098. Agenjo, R. 1947. Catálogo ordenador de los lepidópteros de España. Agrotidae (desde platyptera) y Lymantriidae. 05(1): sin paginar.

099. Ceballos, Gonzalo. 1947. Los insectos en la obra de Cervantes. 05(2): 031-036.

100. Español Coll, Francisco. 1947. Un aspecto de la vida en el Sáhara español. Los coleópteros. 05(2): 037-045.

101. Agenjo, Ramón. 1947. Catálogo ordenador de los lepidópteros de España. Euplagiidae, Lasiocampidae, Syssphingidae, Attacidae (= Saturniidae), Bombycidae, Lemoniidae, Sphingidae y Hesperiidae. 05(2): sin paginar.

102. Varea de Luque, A. 1947. Algunas observaciones lepidopterológicas sobre mis campañas 1946 y 1947. 05(3): 047-053.

103. Español Coll, F. 1947. Los colidíidos ciegos del Mediterráneo occidental (Col.). 05(3): 055-061.

104. Agenjo, R. 1947. Catálogo ordenador de los lepidópteros de España. Lycaenidae, Riodinidae, Libytheidae, Satyridae, Nymphalidae, Pieridae y Papilionidae. 05(3): sin paginar.

105. Ceballos, Gonzalo. 1947. Las sociedades de los insectos y las humanas. 05(4): 063-068.
106. Benítez Morera, Antonio. 1947. El fiero "Scarites". 05(4): 069-071.

107. Fernández, José María. 1947. A la caza del Carabus faustus. 05(4): 073-075.

108. Montada Brunet, Juan. 1947. Coleópteros del macizo de Montseny (Barcelona). 05(4): 077-078.

109. Fernández, José María. 1947. La Acherontia atropos y su parásito específico. 05(4): 079-082.

110. Español Coll, Francisco. 1947. Coleópteros de Lanzarote. 05(5): 083-097.

111. Agenjo, Ramón. 1947. Acerca de la nomenclatura de varias especies del género Adscita Retzius, 1783 (Lep. Anthroc.). 05(6): 099-107.

112. Varea de Luque, A. 1947. Apuntes sobre mariposas del Guadarrama. 05(6): 109-112.

113. Ribbe, Carl. 1948. La fauna lepidopterológica andaluza. 06: 001-082.

114. Español C., Francisco. 1949. Datos para el conocimiento de los tenebriónidos del Mediterráneo occidental. VI. El género Misolampus Latr. en España y Baleares. 07: 001-007.

115. Cobos, Antonio. 1949. Nuevas especies del género Tetragonoschema Thoms. (Col. Buprestidae, Anthaxini). 07: 009-017.

116. Fernández, José $\mathrm{M}^{\mathrm{a}}$. 1949. La Spheniscomyia filiola Loew. y su mimetismo. 07: 019-021.

117. Lagar, Angel. 1949. Notas sobre Dytiscidae. Descripción de una nueva variación de Laccophilus hyalinus De Geer. 07: 023-024.

118. Compte Sart, Arturo. 1949. Hallazgo de la Cicindela melancholica F. en Mallorca. 07: 025026.

119. Español C., Francisco. 1949. Coleópteros no autóctonos observados en Barcelona y sus alrededores inmediatos. 07: 027-041.

120. Español C., Francisco. 1949. Datos para el conocimiento de los tenebriónidos del Mediterráneo occidental. 07: 043-048.

121. Palau, J. M $\mathbf{M}^{\mathrm{a}}$. 1949. Leptinotarsa decemlineata (Col. Chrysomelidae) en Palma de Mallorca. 07: 049-050.

122. Lagar, Angel. 1949. Los hidrocántaros de Vallvidriera (Barcelona). 07: 051-056.

123. Montero, M. 1949. La Saturnia pyri Schiff., y las ondas electromagnéticas. 07: 057-060.

124. Nájera, L. 1949. Sobre la alimentación de las larvas de phebotomos. 07: 061-069.

125. Palau, José $M^{\mathrm{a}}$. 1949. Algunos hemípteros heterópteros de Mallorca. 07: 071-075.

126. Anduiza, R. 1949. Ecos entomológicos desde Bilbao. I. Reblandecimiento de lepidópteros. 07; 077-079.

127. Sala de Castellarnau, Ignacio. 1949. Notas entomológicas en plena naturaleza. 07: 081-091.

128. Fernández, José $\mathrm{M}^{\mathrm{a}}$. 1949. Los Phoridae. Notas biológicas y sistemáticas. 07: 093-098.

129. Ceballos, G. 1949. Citas nuevas de himenópteros. 07: 099-103.

130. Agenjo, Ramón. 1949. Diagnosis de dos nuevos géneros de Larentiinae (Lep. Geom.). 07: 105106. 
131. Gómez-Menor Ortega, Juan. 1950. Hemípteros. 08: 001-014.

132. Agenjo, R. 1950. De re lepidopterológica. 08: 015-018.

133. Flores, Hilario. 1950. Acerca de la atración sexual a distancia en los lepidópteros. 08: 019-022.

134. Bullón, A. 1950. La oruga de Graëllsia isabelae (Graells) en Cercedilla (Madrid). 08: 023-026.

135. Anónimo. 1950. Consultas. Caza de Vanessa antiopa (L.) en la sierra de Guadarrama. Sobre Zegris eupheme. Sobre duración en extendedor de mariposas. Como cazar Plebejus. Zephyrus hesperica. 08: 027-031.

136. Agenjo, R. 1950. Reseña de capturas. (I). 08: 033034.

137. Anónimo. 1950. Noticiario lepidopterológico. 08: 035-037.

138. Pardo Alcaide, Anselmo. 1950. Los géneros de Meloidae de la fauna hespérica. 08: 039-079.

139. Español, Francisco. 1950. Coleópteros de las Pitiusas (Baleares occidentales) (Fam. Scarabaeidae). 08: 081-089.

140. Fernández, José Ma $\mathrm{M}^{\mathrm{a}}$ 1950. Coleópteros canarios. Fáunula de la isla de La Palma. 08: 091-103.

141. Zarco, E. 1950. Insectos y micosis. Ips acuminatus Gyll. (Col. Scoly.) en la pudrición azul de la madera. 08: 105-112.

142. Zarco, E. 1950. Karl Escherich (1871-1951). 08: 113-115.

143. Agenjo, Ramón (R.A.). 1950. Las rosquillas negras. 08: 117-119.

144. Anónimo. 1950. Sección bibliográfica. 08: 121-130.

145. Español, F. 1950. Reseña bibliográfica de: G Colas, Guide de l'Entomologie. 08: 131.

146. Español, F. 1950. Reseña bibliográfica de: J. Bechyne, Contribution à la connaissance du genre Timarcha Latr. 12: Etudes phyllogénétiques et zoogéographiques (col. Phytophaga, Chrysomelidae). 08: 131.

147. Español, F. 1950. Reseña bibliográfica de: L. E. Peña Guzmán, Insectos: caza, conservación, clasificación. 08: 132

148. Español, F. 1950. Reseña bibliográfica de: H Normand, La chasse aux coléoptères en Tunisie. 08: 132

149. Agenjo, R. 1950. Reseña bibliográfica de: N. Obraztsov, Versuch einer systematischen übersicht der europäischen Eucosmini-Gattungen (Lepidoptera, Tortricidae). 08: 132-134.

150. Agenjo, R. 1950. Reseña bibliográfica de: L. G. Higgins, Butterflies in Granada. 08: 134-135.

151. Gómez-Menor, Juan. 1951. Homópteros. 09: 001013.

152. Agenjo, Ramón. 1951. El IX Congreso internacional de Entomología. Amsterdam, 1951. 09: 015-030.

153. Alfaro Arregui, Pedro. 1951. Así capturé la "Aglia tau". 09: 031-033.

154. Agenjo, Casilda. 1951. La Argynnis paphia (L.) en Andalucía y algunos datos sobre los lepidópteros de Siles, en la Sierra de Segura, provincia de Jaén. 09: 035-040.
155. Agenjo, R. 1951. Algunos lepidópteros de Andújar (provincia de Jaén). 09: 041-045.

156. Varea de Luque, A. 1951. Consultas. No 4: Sobre variedades de Zerynthia rumina. $\mathrm{N}^{\mathrm{o}}$ 5: Sobre cría de Arctia hebe. $\mathrm{N}^{\mathrm{o}}$ 6: Sobre la recolección con lámparas de vapores de mercurio. $\mathrm{N}^{\mathrm{o}} 7$ : Sobre Plebejus (Lysandra) amandus. 09: 047-052.

157. Agenjo, R. 1951. Reseña de capturas. (II). 09: 053-057.

158. Agenjo, R. 1951. Noticiario lepidopterológico, II. 09: 059-061.

159. Español C., Francisco. 1951. Datos para el conocimiento de los Tenebriónidos del Mediterráneo occidental. IX: Comentario biogeográfico sobre los Crypticus ibéricos. 09: 063-070.

160. Español C., Francisco. 1951. Elaphrus lheritieri Ant., carábido nuevo para la fauna ibérica. 09: 071-073.

161. Lagar Mascaró, Angel. 1951. Los hidrocántaros de la delta del río Llobregat. 09: 075-080.

162. Fernández, José María. 1951. Anopheles (Myzomyia) hispaniola Theob. en la isla de La Palma. 09: 081-087.

163. Báguena Corella, Luis. 1951. Los insectos perjudiciales a la agricultura en los territorios españoles del Golfo de Guinea. 09: 089-124

164. Agenjo, R. 1951. Plaga de la garrofa almacenada, producida por dos especies de Ephestia Gn. (Lep. Phycitidae). 09: 125-129.

165. Ceballos, G. 1951. Lo inexplicable. 09: 131-135.

166. Rupérez Cuellar, Adolfo. 1951. El ultrasonido en la vida de los insectos. 09: 137-147.

167. Anónimo. 1951. Sección bibliográfica. 09: 149-157.

168. Agenjo, Ramón. 1951. Reseña bibliográfica de: E.M. Hering, Biology of the leaf Miners 09: 159160.

169. Agenjo, Ramón. 1951. Reseña bibliográfica de: F. J. Aubert (F.J.), Papillons d'Europe. 09: 160-162.

170. Agenjo, Ramón. 1951. Catálogo ordenador de los lepidópteros de España. Geometridae. 09: sin paginar.

171. Mateu, J. 1952. Las capturas del Pseudomasoreus canigoulensis Fairm. Laboulb. (Col. Carabidae) en España. 10: 001-004.

172. Español Coll, Francisco. 1952. Datos para el conocimiento de los tenebriónidos del Mediterráneo occidental. X: Los Gonocephalum de España. 10: 005-014.

173. Lagar, Angel. 1952. Notas sobre Haptoderus (Col. Carab.) de los Pirineos catalanes. 10: 015-018.

174. Español Coll, Francisco. 1952. Datos para el conocimiento de los tenebriónidos del Mediterráneo occidental. XI: Los Cossyphus ibéricos. 10: 019027.

175. Ceballos, Gonzalo. 1952. Himenópteros notables de la fauna española. 10: 029-039.

176. Gómez-Menor Ortega, Juan. 1952. Las especies de Rhinocoris y Sphedanolestes (Hem. Reduviidae) de España. 10: 041-045.

177. Fernández, José M. 1952. La Lampromyia canariensis Macq. 10: 047-055. 
178. Morales Agacino, E. 1952. El centro de investigaciones antiacridianas de Londres. 10: 057-066, láminas I-IV.

179. Báguena, Luis. 1952. Algunas notas sobre entomología médico-legal. 10: 067-101.

180. Zarco, E. 1952. Los insectos de la madera en el Africa occidental española. 10: 103-114, láminas V-IX.

181. Agenjo, R. 1953. Subespecies nuevas de lepidópteros santanderinos. 11: 001-006.

182. Agenjo, Casilda. 1953. La variabilidad de la isabelae (Graells.) (Lep. Syssph.). 11: 007-010.

183. Pardo Alcaide, Anselmo. 1953. Genera de Coleópteros de la fauna ibérica 2. Familia Cleridae. 11: 011-022.

184. Lagar Mascaró, Angel. 1953. Algunos Dytiscidae interesantes de la fauna ibero-balear. 11: 023-025.

185. Español, Francisco. 1953. Las Oberea de Cataluña (Col. Cerambycidae). 11: 027-032.

186. Lagar Mascaró, Angel. 1953. Los Scarabaeoidea (Col.) del Delta del río Llobregat. 11: 033-040.

187. Agenjo, R. 1953. Un microlepidóptero murciano género y especie nuevos de la familia Scythridae. 11: 041-047.

188. Anónimo. 1953. Noticiario entomológico. II Reunión Internacional para las investigaciones de la lucha biológica. 11: 049-052.

189. Agenjo, R. 1954. R. P. Ambrosio Fernández, O.S.A. (1882-1953). 12: 001-019.

190. Agenjo, R. 1954. D. Manuel Pujol y Fiol (18751953). 12: 021-028.

191. Agenjo, Ramón. 1954. Reseña de capturas. (III). 12: 029-036.

192. Smith, K. M. 1954. Noticiario entomológico. Enfermedades de virus en los insectos. 12: 037-042.

193. Docavo, Ignacio. 1955. Contribución al conocimiento de los Braconidae de España. 13: 001-034.

194. Reglat, Jean-François. 1955. Excursión a Pinares Llanos (3 de junio de 1950). 13: 035-041.

195. Ruaix, Angelita. 1955. En busca de la Nymphalis antiopa (Lep. Nymph.). 13: 043-046.

196. Varea de Luque, A. 1955. Consultas. No 8: Sobre las especies españolas de Satyrus. N ${ }^{\circ}$ 9: Sobre Chrysophanus dispar. $\mathrm{N}^{\mathrm{o}}$ 10: Sobre T. A. Chapman en España. 13: 047-051.

197. Agenjo, Ramón. 1955. Noticiario lepidopterológico. 13: 053-058.

198. Agenjo, Ramón. 1955. Catálogo ordenador de los lepidópteros de España. Carposinidae, Tortricidae y Phaloniidae. 13: sin paginar.

199. Español C., Francisco. 1956. Los erotílidos (Col.) del macizo del Montseny (Barcelona). 14: 001012 .

200. Pardo Alcaide, Anselmo. 1956. Analectas Entomológicas. V. Los Zonitis de la fauna ibérica (Col. Meloidae). 14: 013-019.

201. Pardo Alcaide, Anselmo. 1956. Analectas Entomológicas. VI. Los Cerocoma de la fauna ibérica (Col. Meloidae). 14: 021-027.

202. Báguena, Luis. 1956. Dos especies nuevas más de Scarabaeidae de la fauna española. 14: 029-030.
203. Morales Morales, E. 1956. Algunas curiosidades americanas sobre los saltamontes y las langostas. 14: 031-040.

204. Peris, Salvador V. 1956. Notas sobre Acemyiini (Dip. Tachinidae). 14: 041-047.

205. Benítez Morera, Antonio. 1956. Sobre una emigración de odonatos y de un ingenioso procedimiento para cazarlos. 14: 049-050.

206. Ceballos, G. 1956. Un Centenario. 14: 051-054.

207. Uvarov, B. P. 1956. La langosta del desierto y su ambiente. Extracto de la conferencia pronunciada en el Instituto Español de Entomología en el mes de Enero de 1955, realizado por E. Zarco. 14: 055-061.

208. Anónimo. 1956. Consultas. Lista de hemípteros de Palma de Mallorca enviados para su determinación por don Arturo Compte Sart, clasificados por J. G. Menor. 14: 063-071.

209. Agenjo, Ramón. 1956. Consideraciones sobre el estudio de las fórmulas cromosómicas en los lepidópteros y sobre todo en el complejo de formas del grupo Plebejus (Lysandra) coridon (Poda), con la descripción de tres nuevas subespecies. 14: 073-087.

210. Agenjo, Ramón. 1956. Reseña bibliográfica.de: R. Schwarz, Motyli (en checo) (Mariposas). 14: 089-090.

211. Agenjo, Ramón. 1956. Reseña bibliográfica.de: B. J. Lempke, Catalogus der Nederlandse Macrolepidoptera. 14: 090-092.

212. Instituto Español de Entomología. 1957. Introducción. 15: 003.

213. Agenjo, R. 1957. Monografía de las especies españolas de la familia Lymantriidae Hampson, 1892, con especial referencia a las de interés forestal (Lepidoptera). 15: 005-143, láminas I-XI.

214. Báguena Corella, Luis. 1957. Notas sobre ecología y etología de los Scarabaeoidea ibéricos de interés forestal. 15: 145-173.

215. Gómez-Menor Guerrero, Juan Miguel. 1957. Biocenosis en las agallas del chopo. 15: 175-186.

216. Gómez-Menor Guerrero, Juan Miguel. 1957. Datos biológicos del Ecacanthothrips nodicornis (Reut.). 15: 187-192.

217. Español Coll, F. 1958. Sobre algunos endomíquidos de Cataluña (Col. Cucujoidea). 16: 003-012.

218. Pardo Alcaide, A. 1958. Analectas Entomológicas. VII. Los Sitarini de la Península Ibérica (Col. Meloidae). 16: 013-022.

219. Cobos Sánchez, A. 1958. Datos para el catálogo de los Coleópteros de España. Especies de los alrededores de Málaga. (Adiciones y correcciones a la lista de 1949). 16: 023-028.

220. Lagar Mascaró, A. 1958. Coleópteros de la delta del río Llobregat. 16: 029-032.

221. Codina Padilla, F. 1958. Las Lachnaea Redt. ibero-marroquíes (Col. Chrysomelidae). 16: 033044.

222. Templado, Joaquín. 1958. Smicromyrme viduata Pall. parásito de Bembex mediterranea Handl. 16: 045-047.

223. Peris Torres, S. V. 1958. Sobre las formas canarias 
de Solva Walk. (Dipt., Xylophagidae). 16: 049-051.

224. Agenjo, R. 1958. Dos Laspeyresia dañadoras de pinos y abetos. 16: 053-058, lámina I.

225. Cadahía Cicuendez, D. 1958. Los Aegeridae y los chopos. 16: 059-074, láminas II-III.

226. Cañizo Gómez, J. del. 1958. Los insectos en el "folklore". 16: 075-078.

227. Docavo Alberti, I. 1958. Al habla con la mosca mediterránea. Guión radiofónico. 16: 079-084.

228. Agenjo, Ramón. 1958. Lepidópteros de Tortosa, provincia de Tarragona, recolectados por D. Eugenio Balaguer en los años 1932 y 1933. 16: 085-095.

229. Heath, J.\& Smith, C. C. 1958. Lepidópteros del Alto Aragón. Graellsia 16: 097-105.

230. Español Coll, Francisco. 1958. Datos para el conocimiento de los tenebriónidos del Mediterráneo occidental (Coleoptera). 16: 107-116.

231. Peris Torres, S. V. 1958. Una colección de Syrphidae de Santander (Diptera). 16: 117-119.

232. Gómez-Menor, J. 1958. Homópteros Sternorryncha que atacan a la encina. Primera parte. Serie Dimera. 16: 121-197.

233. Docavo Alberti, I. 1958. Al habla con la mosca mediterránea. Guión radiofónico (segunda parte). 16: 199-204.

234. Agenjo, R. 1959. Catascia dognini (ThierryMieg, 1910), especie nueva para la Península Ibérica (Lep. Geom.). 17: 003-005.

235. Agenjo, R. 1959. La polilla de las garrofas, plaga actual de las naranjas (Lep. Phycit.). 17: 007-017, láminas I-II.

236. Agenjo, R. 1959. Unas pocas mariposas valencianas. 17: 019-028

237. Báguena Corella, L. 1959. Notas sobre ecología y etología de los Scarabaeoidea ibéricos de interés en edafología (Coleoptera). 17: 029-044.

238. Cobos Sánchez, A. 1959. Notas sobre Chrysomelidae ibéricos (Coleoptera). 17: 045-051.

239. Español Coll, F. 1959. Algunos bostríquidos de la Guinea española (Col. Cucujoidea). 17: 053-057.

240. Español Coll, F. 1959. Datos para el conocimiento de los tenebriónidos del Mediterráneo occidental. XIV, los Melanimini de la Península Ibérica y Marruecos (Coleoptera). 17: 059-070.

241. Winkler, J. R. 1959. Trichodes crabroniformis hidalgo, nueva subespecie de clérido de España (Coleoptera). 17: 071-075.

242. Ebner, R. 1959. Sobre la presencia y variabilidad de Eulithinus analis (Ramb.) (Dermaptera). 17: 077-082.

243. Docavo Alberti, I. 1959. El grillo campestre. Guión radiofónico. 17: 083-088.

244. Anónimo. 1959. Reseña bibliográfica de: J. Aubert, Plecoptera. 17: 089-090.

245. Anónimo. 1959. Reseña bibliográfica de: L. Berland, Atlas des Hyménoptères de France, Belgique et Suisse. 17: 090.

246. Anónimo. 1959. Reseña bibliográfica de: $\mathrm{H}$ Lorenz \& M. Kraus, Die Larvalsystematik der Blattwespen (Tenthredinoidea und Megalodon- toidea). 17: 090-091.

247. Anónimo. 1959. Reseña bibliográfica de: V. B. Wigglesworth, Physiologie des insectes. 17: 091.

248. Báguena, Luis. 1959. Los Rhizotrogina ibéricos (Col. Scarab.). 17: 093-120.

249. Ceballos, Pedro. 1959. Las especies españolas del género Leucospis (Hym. Chalc.). 17: 121-127.

250. Compte Sart, Arturo. 1959. Sobre los esfécidos de Mallorca. 17: 129-140.

251. Gómez-Menor, J. 1959. Homoptera Sternorryncha que atacan a la encina. Segunda parte. Serie Monomera. 17: 141-201.

252. Docavo Alberti, I. 1959. La vida de la Graellsia isabelae. Guión radiofónico. 17: 203-209.

253. Anónimo. 1959. Reseña bibliográfica de: K. Werner, Die Larvalsystematik einiger kleinschmetterlingfamilien (Hyponomeutidae, Orthoteliidae, Acrolepiidae, Tineidae, Incurvariidae und Adelidae). 17: 211

254. Anónimo. 1959. Reseña bibliográfica de: B. Swatschek, Die Larvalsystematik der Wickler (Tortricidae und Carposinidae). 17: 211-212.

255. Anónimo. 1959. Reseña bibliográfica de: A. D. Imms, Outlines of Entomology. 17: 212-213.

256. Anónimo. 1959. Reseña bibliográfica de: M. I. Constantineanu, Fauna Republicii Populare Romîne: Insecta, vol. IX, fasc. 4; Familia Ichneumonidae, subfamilia Ichneumoninae, tribu Ichneumoninae Stenopneusticae. 17: 213.

257. Báguena, Luis. 1960. Los Donaciinae ibéricos de la colección del Instituto Español de Entomología (Col. Chrysomelidae). 18: 003-014.

258. Báguena, Luis. 1960. Notas críticas y descriptivas sobre algunos Clytrini (Col. Chrysomelidae). 18: 015-028.

259. Báguena, Luis. 1960. Sobre Scarabaeidae de España y Marruecos. 18: 029-035.

260. Codina Padilla, F. 1960. Apuntes sobre Chrysomelidae ibero-marroquíes. 18: 037-050.

261. Español Coll, F. 1960. Los Carabus de la provincia de Tarragona (Col. Carabidae). 18: 051-058.

262. Peris, S. V. 1960. Notas dipterológicas. 18: 59-63.

263. Ceballos, G. 1960. Himenópteros nuevos para la fauna española y citas de nuevas procedencias provinciales de especies. 18: 065-071.

264. Compte Sart, Arturo. 1960. Biografía de la Selysiothemis nigra V. d. L. (Odonatos). 18: 073115.

265. Docavo Alberti, I. 1960. El Alacrán cebollero. Guión radiofónico. 18: 117-122.

266. Báguena, Luis. 1960. Clave práctica para la determinación de los Clytrini ibéricos (Col. Chrysomelidae). 18: 123-145.

267. Báguena, Luis. 1960. Los Troginae ibéricos (Col. Scarabaeidae). 18: 147-151.

268. Español Coll, F. 1960. Los Trichodes ibéricos (Col. Cleridae). 18: 153-164.

269. Flores, H. 1960. "Sinoxylon sexdentatus" insecto perforador del plomo (Col. Bostrichidae). 18: 165-173.

270. Rupérez, Adolfo. 1960. Observaciones sobre el 
mecanismo de enrollamiento de hojas de frondosas por orugas de tortrícidos. 18: 175-178.

271. Docavo Alberti, Ignacio. 1960. La vida de la cigarra. Guión radiofónico. 18: 179-185.

272. Ceballos, Gonzalo. 1960. Reseña bibliográfica de: H. Townes \& M. Townes, Ichneumon-Flies of America North of Mexico: 2. Subfamilies Ephialtinae, Xoridinae, Acaenitinae. 18: 187-188.

273. Agenjo, R. 1961. Morfología y distribución geográfica en España de Hipparchia alcyone (Schiff. 1776) (Lep. Satyridae). 19: 003-010.

274. Ceballos, G. 1961. Especies de Ichneumonidae nuevas para la fauna española (Hymenoptera). 19: 011-018.

275. García de Viedma, Manuel. \& Suárez, Francisco J. 1961. Un nuevo Xeris de Marruecos (Hym. Siricidae). 19: 019-025, láminas I-II.

276. Cobos, A. 1961. Notas sobre Chrysomelidae ibéricos (Coleoptera). 19: 027-043.

277. Codina Padilla, Francisco. 1961. Apuntes sobre Chrysomelidae ibero-marroquíes (Coleoptera). 19: 045-057.

278. Codina Padilla, Francisco. 1961. Nota sobre las diversas formas del subgénero Barathraea Lacordaire (Col. Chrysomelidae). 19: 059-068.

279. Codina Padilla, Francisco. 1961. Notas sobre los Luperus marroquíes del subgénero Calomicrus Stephens (Col. Chrysomelidae). 19: 069-079.

280. Español, Francisco. 1961. El género Tarphius, nuevo para la fauna española (Col. Colydiidae). 19: 081-085

281. Lagar Mascaró, Angel. 1961. Notas sobre Dytiscidae VII. Especies críticas o interesantes de la fauna ibérica (Coleoptera). 19: 087-089.

282. Aubert, J. 1961. Los plecópteros del Instituto Español de Entomología. 19: 091-094.

283. Flores, H. 1961. La princesa Yama-Mai. 19: 095 099.

284. Docavo Alberti, Ignacio. 1961. La vida de la cigarra (continuación). Guión radiofónico. 19: 101-107.

285. Anónimo. 1961. Reseña bibliográfica de: H. Beck, Die Larvalsystematik der Eulen (Noctuidae). 19: 109.

286. Anónimo. 1961. Reseña bibliográfica de: I. Docavo Alberti, Los géneros de bracónidos de España. 19: 110

287. Anónimo. 1961. Reseña bibliográfica de: I. Hasenfuss, Die Larvalsystematik der Zünsler (Pyralidae). 19: 110-111.

288. Anónimo. 1961. Reseña bibliográfica de: R. B. Selander, Bionomics, Systematics and Phylogeny of Lytta, a Genus of Blister beetles (Coleoptera, Meloidae). 19: 111-112.

289. Diniz, Manuel de Assunçao. 1962. Claves para la identificación de los géneros de ápidos de la Península Ibérica. 19: 113-135, láminas III-XIV.

290. Báguena, Luis \& Breuning, Stephan. 1962. Catálogo de los Cerambycidae de las provincias españolas del Golfo de Guinea (Coleoptera). 19: 137-228.

291. Español Coll, F. 1962. Tenebriónidos del Médano
(Tenerife) (Coleoptera). 19: 229-235.

292. Capilla Caballero, Juan. 1962. Secretillos de laboratorio. 19: 237-243.

293. Docavo Alberti, I. 1962. La hormiga-león. Guión radiofónico. 19: 245-253.

294. Anónimo. 1962. Reseña bibliográfica de: A. S. Balachowsky, Entomologie appliquée à l'Agriculture. Tome I, Coléoptères (1er vol.) (Caraboidea, Staphylinoidea, Hidrophiloidea, Scarabaeoidea, Dascilloidea, Cantharoidea, Bostrychoidea, Cucujoidea et Phytophagoidea). 19: 255-256.

295. Anónimo. 1962. Reseña bibliográfica de: L. Berland, Atlas des Néuroptères de France. 19: 256-257.

296. Anónimo. 1962. Reseña bibliográfica de: P. Blüthgen, Die Faltenwespen Mitteleuropas (Hymenoptera, Diploptera). 19: 257-258.

297. Anónimo. 1962. Reseña bibliográfica de: Internacional Code of Zoological Nomenclature adopted by the XV International Congress of Zoology. 19: 259.

298. Agenjo, R. 1963. Algunos lepidopteros de Punta Umbría, provincia de Huelva. La canaria Eilema albicosta (Rghfr., 1894) nueva para España y Europa (Lepidoptera). 20: 003-020, lámina I.

299. Agenjo, R. 1963. El microlepidóptero etiópico Daraba laisalis (Wlk., 1859) plaga del pimiento y la berenjena en Somalia, hallado en Punta Umbría, provincia de Huelva, nuevo para la fauna paleártica (Lep. Pyraustidae). 20: 023-028.

300. Lajonquière, Yves. de. 1963. Captura en Almería de un Cossidae nuevo para la fauna europea (Lepidoptera). 20: 029-031.

301. Ruano, Ramiro. G. 1963. Nota técnica para la recolección de huevos de Ephestia kuhniella Zell. (Lepidoptera). 20: 033-035.

302. Ceballos, G. 1963. Los anomalinos de España (Himenoptera). 20: 037-054.

303. Ceballos Jiménez, Pedro. 1963. Sirícidos españoles (Himenoptera). 20: 055-067.

304. Codina Padilla, F. 1963. Apuntes sobre Chrysomelidae ibero-marroquíes (Coleoptera). 20: 069-077.

305. Codina Padilla, F. 1963. Un nuevo Phaedon Latr., marroquí (Col. Chrysomelidae). 20: 079-082.

306. Compte Sart, Arturo. 1963. Los Oedemeridae de las Islas Baleares (Coleoptera). 20: 083-109.

307. Dicenta, A. \& Balcells, E. 1963. Notas ecológicas: Chrysolina (= Chrysomela) banksi F. (Col. Chrysomelinae). 20: 111-117.

308. Español, F. 1963. Sobre algunos Cucujidae españoles (Coleoptera). 20: 119-124.

309. Leclercq, Marcel. 1963. Syrphidae de España. 20: 125-129.

310. Ceballos, Gonzalo. 1963. Un libro peligroso. 20: 131-140.

311. Docavo Alberti, I. 1963. Las cucarachas. Guión radiofónico. 20: 141-146.

312. Anónimo. 1963. Reseña bibliográfica de: A. S. Balachowsky, Entomologie appliquée à l'Agriculture. Tome I, Coleoptères (2.éme vol.) 
(Phytophagoidea suite et fin, Chrysomelidae, Curculionidae, Attelabidae, Scolytidae et Platypodidae). 20: 147-148.

313. Anónimo. 1963. Reseña bibliográfica de: E. J. Fittkau, Die Tanypodinae (Diptera: Chironomidae). 20: 148 .

314. Agenjo, Ramón. 1964. El XII Congreso Internacional de Entomología. Londres, 1964. 20: 149-162.

315. Agenjo, R. 1964. Los nombres vulgares de las mariposas españolas. 20: 163-190.

316. Agenjo, Ramón. 1964. Sección de capturas. (IV) 20: 191-202.

317. Arozarena Doblado, Rafael. 1964. Eumenidae de las islas Canarias (Hymenoptera). 20: 203-211.

318. Compte Sart, Arturo. 1964. Sobre un Polistes gallicus teratomorfo (Hymenoptera). 20: 213-224.

319. Llopis Minguez, Baltasar. 1964. Contribución al conocimiento de los icneumónidos de España. Especies nuevas para la fauna española y citas de nuevas localidades (Hymenoptera). 20: 225-229.

320. Vergés Serra, F. 1964. Algunos ápidos capturados en el término de Canet de Mar (Barcelona). Primera parte (Hymenoptera). 20: 231-244.

321. García de Viedma, Manuel. 1964. Larvas de coleópteros. 20: 245-275.

322. Español, F. 1964. Algunos carábidos interesantes del Levante español (Coleoptera). 20: 277-280.

323. Español, F. 1964. Las Lagria de España (Col. Heteromera). 20: 281-286.

324. Español, F. 1964. Notas sobre anóbidos (Coleoptera). 20: 287-299.

325. Lagar Mascaró, Angel. 1964. Algunos carábidos nuevos o interesantes de la provincia de Tarragona (Coleoptera). 20: 301-306.

326. Docavo Alberti, Ignacio. 1964. Un científico que sorprende: el entomólogo. Guión radiofónico. 20 307-313.

327. Anónimo. 1964. Reseña bibliográfica de: W. Derksen \& U. Scheiding, Index Literaturae Entomologicae; t. I (A-E). 20: 315.

328. Anónimo. 1964. Reseña bibliográfica de: C. F. Dos Passos, A synonymic list of the Nearctic Rhopalocera. 20: 315-316.

329. Anónimo. 1964. Reseña bibliográfica de: C. Jeuniaux, Chitine et chitinolyse. 20: 316.

330. Anónimo. 1964. Reseña bibliográfica de: J. Missonnier, Etude écologique du développement nymphal de deux Diptères Muscides phytophages: Pegomyia betae Curtis et Chortophila brassicae Bouché. 20: 316-317

331. Anónimo. 1964. Reseña bibliográfica de: S. H. Skife, Las hormigas. 20: 317.

332. Agenjo, R. 1964. Catálogo ordenador de los lepidópteros de España. Opostegidae, Nepticulidae, Tischeriidae, Crinopterygidae, Heliozelidae, Tineidae, Ochsenheimeriidae, Lyonetiidae. 20: $\sin$ paginar.

333. Agenjo, R. 1965. Caza de mariposas con reclamo. 21: 003-008, lámina I.

334. Agenjo, R. 1965. Un neotipo para la Graellsia isa- belae (Grlls. 1849) (Lep. Syssphingidae). 21: 009010 .

335. Ceballos, G. 1965. El género Helorus Latr. (Hym. Proctotrupoidea). 21: 011-016.

336. Ceballos, Gonzalo. 1965. Novedades en himenópteros de España y comentarios sobre algunas especies. 21: 017-023.

337. Docavo Alberti, Ignacio. 1965. Nuevas aportaciones al conocimiento de los Dacnusini de España (Hym. Braconidae). 21: 025-039.

338. Báguena, Luis. 1965. Los grandes Silphidae ibéricos (Coleoptera). 21: 041-054.

339. Cobos, A. 1965. Adiciones y correciones al "Ensayo sobre los Asida Latr., de Marruecos" (Col. Tenebrionidae). 21: 055-057.

340. Cobos, A. 1965. Una nueva especie de Helioctamenus de Canarias (Col. Colydiidae). 21: 059-063.

341. Español, F. 1965. Sobre la distribución de los tenebriónidos en la mitad norte del litoral levantino español (Coleoptera). 21: 065-077.

342. Lagar Mascaró, Angel. 1965. Los Carabus de las Islas Canarias (Col. Carabidae). 21: 079-084.

343. Santoro, F. H. \& G. de Viedma, M. 1965. Los líctidos del Instituto Español de Entomología (Coleoptera). 21: 085-087.

344. Fernandes, J. de A. 1965. Dermápteros de Portugal. 21: 089-094.

345. Ragge, D. R. 1965. Ortópteros y dermápteros colectados en la Península durante los años de 1962 y 1963 por misiones del British Museum (Natural History). 21: 095-119.

346. Carmona, María Manuela. 1965. O problema dos ácaros nas plantas cultivadas em Portugal. 21: 121-130.

347. Azevedo Silva, Francisco \& Serrão Nogueira, Carlos David. 1965. O género Periclista Konow na entomofauna do Sobreiro em Portugal (Hym. Tenthredinidae). 21: 131-141.

348. Arroyo Varela, M. 1965. La "Fondazione Filippo Silvestri”. 21: 143-145.

349. Docavo Alberti, Ignacio. 1965. El escarabajo pelotero. Guión radiofónico 21: 147-157.

350. Anónimo. 1965. Reseña bibliográfica de: V. M. Dirsh, The African genera of Acridoidea. 21: 159160.

351. Anónimo. 1965. Reseña bibliográfica de: C. Ferrière, Hymenoptera Aphelinidae díEurope et du Bassin Méditerranéen. 21: 160-161.

352. Anónimo. 1965. Reseña bibliográfica de: $M$. Beier, Ordnung Pseudoscorpionidae. 21: 161-162.

353. Anónimo. 1965. Reseña bibliográfica de: $\mathrm{H}$. Priesner, Ordnung Thysanoptera. 21: 162-163.

354. Anónimo. 1965. Reseña bibliográfica de: K. Princis, Ordnung Blattariae. 21: 163.

355. Anónimo. 1965. Reseña bibliográfica de: D. R Ragge, Grasshoppers, Crickets \& Cockroaches of the British Isles. 21: 163-164.

356. Anónimo. 1965. Reseña bibliográfica de: A. Vandel, Biospeleology: The Biology of cavernicolous animals. 21: 164-165.

357. Agenjo, Ramón. 1965. Catálogo ordenador de los lepi- 
dópteros de España. Micropterygidae, Eriocraniidae, Hepialidae, Adelidae, Lithocolletidae, Argyresthiidae, Plutellidae, Epermeniidae, Coleophoridae (= Eupistidae), Elachistidae, Douglasidae. 21: sin paginar.

358. González López, Francisco. 1966. La Argynnis paphia en Albacete y algunos datos para el conocimiento de los lepidópteros del nacimiento del río Mundo en la sierra de Segura, provincia de Albacete. 22: 003-006.

359. Diniz, M. de Assunção. 1966. A propósito da data de publicação do "Catalogue des insectes du Portugal" de Paulino d'Oliveira (Coleoptera). 22: 007-012.

360. Español, F. 1966. Dos años de actividades bioespeleológicas en el noreste de España (Coleoptera). 22: 013-023.

361. Ferreira, Raúl do Nascimento. 1966. Casos teratológicos atrofia do tarso posterior de um Oryctes nasicornis (Coleoptera). 22: 025-027.

362. Lagar Mascaró, Angel. 1966. Una nueva especie de Speophilus de la provincia de Barcelona (Col. Catopidae). 22: 029-032.

363. Pardo Alcaide, Anselmo. 1966. Notas sobre Malachiidae del Mediterráneo occidental (Coleoptera). 22: 033-045.

364. Arozarena Doblado, Rafael. 1966. Nuevas citas para Canarias de la subfamilia Encirtinae (Hymenoptera). 22: 047-056.

365. Mingo Pérez, Elvira. 1966. Los Oxybelini de la Península Ibérica (Hymenoptera). 22: 057-121.

366. Harz, Kurt. 1966. La chicharra Ephippiger ephippiger Fieb., y sus razas (Orth. Tettigoniidae). 22: 123-133.

367. Docavo Alberti, Ignacio. 1966. La oruga de las coles. Guión radiofónico. 22: 135-144.

368. Anónimo. 1966. Reseña bibliográfica de: M. Reitter, Coleópteros, la enigmática vida de los escarabajos. 22: 145-146.

369. Anónimo. 1966. Reseña bibliográfica de: B. P. Uvarov, Grasshoppers and Locusts. 22: 145-146.

370. Agenjo, R. 1966. Catálogo ordenador de los lepidópteros de España. Yponomeutidae, Ethmiidae, Scythrididae, Pterolonchidae, Chryptophasidae, Oecophoridae, Blastoblasidae, Momphidae (= Cosmopterygidae), Aegeriidae, Heliodinidae (= Schreckensteiniidae), Glyphipterygidae, Alucitidae (= Orneodidae). 22: sin paginar.

371. Agenjo, Ramón. 1966. "Mariposas", "Falenas" "Polillas" y "Micros". 22: 147-155.

372. Ceballos, G. 1966. Himenópteros nuevos para la fauna española y nuevas localidades de especies ya citadas. 22: 157-165.

373. Español, F. 1966. Datos para el conocimiento de los tenebriónidos del Mediterráneo occidental (Coleoptera). 22: 167-175.

374. Pardo Alcaide, Anselmo. 1966. Notas sobre Dasytidae ibero-mauritanos (Coleoptera). 22: 177-189.

375. Carmona, María Manuela \& Alvim, Henrique de Sousa. 1966. Nota sobre os parasitas do Prays oleaellus (F.) em Portugal. 22: 191-196.

376. Nogueira, C. D. Serrão \& Cabral, M. T. E.
Cardoso. 1966. Tortrix viridana L. nos montados de Sobro; Disminuição da população na fase de pupa. 22: 197-207.

377. Morales Agacino, E. 1966. El laboratorio de entomología de la Sociedad "Aranzadi" de Ciencias Naturales. 22: 209-212.

378. Docavo Alberti, Ignacio. 1966. El mosquito común. Guión radiofónico. 22: 213-223.

379. Anónimo. 1966. Reseña bibliográfica de: G. Domenichini, Hym. Eulophidae, Palearctic Tetrastichinae. 22: 225-226.

380. Anónimo. 1966. Reseña bibliográfica de: G. Guennelon, Contribution à l'étude de la diapause embryonnaire chez Archips rosana L. (Lepidoptera - Tortricidae). 22: 226.

381. Anónimo. 1966. Reseña bibliográfica de: W. Derksen \& U. Scheiding-Göllner, Index Literaturae Entomologicae, t. II (F-L). 22: 226.

382. Agenjo, Ramón. 1966. Catálogo ordenador de los lepidópteros de España. Psychidae, Symmocidae, Galleriidae, Crambidae, Phycitidae, Pyralidae, Pyraustidae, Pterophoridae, Endromididae. Epílogo. 22: sin paginar.

383. Agenjo, Ramón. 1967. Recordatorio y retrato del Excmo. Sr. Prof. Dr. D. Gonzalo Ceballos y Fernández de Córdoba, Director de Graellsia y del Instituto Español de Entomología, fallecido el 4 de marzo de 1967. 23: 001-003.

384. Agenjo, R. 1967. La gran invansión de lepidópteros, ocurrida en Madrid en la tercera semana de Mayo de 1966. 23: 005-008, lámina I.

385. Agenjo, R. 1967. Tentativa de reaclimatación en Madrid de la mariposa del madroño. 23: 009-013, láminas II-III.

386. Agenjo, R. 1967. Sección de capturas. (V). 23: 015-026, lámina IV.

387. Agenjo, R. 1967. In memoriam. El Prof. Dr. Erich Martin Hering (1893-1967). 23: 027-033.

388. Agenjo, R. 1967. In memoriam. Nicholas Sergeevich Obraztsov (1906-1966). 23: 035053 .

389. Baraud, Jacques. 1967. Contribución al estudio de las Hoplia de España (Col. Scarabaeoidea). 23: 055-063.

390. Español, F. 1967. Los Platycerus Fourcroy del Mediterráneo occidental (Col. Lucanidae). 23: 065-070.

391. Lagar Mascaró, Angel. 1967. Coleópteros del delta del río Llobregat. VI Nota. 23: 071-079.

392. Mingo, Elvira. 1967. Adición a los Psenini de España (Hym. Sphecidae). 23: 081-092.

393. Vergés Serra, F. 1967. Euménidos capturados en la comarca de Canet de Mar, provincia de Barcelona (Hym., Diploptera). 23: 093-110.

394. Van der Godt, V. S. \& Lucas, J. A. W. 1967. Recolección de sírfidos en Albarracín, provincia de Teruel, durante el verano de 1965 (Dipt. Syrphidae). 23: 111-119.

395. Agenjo, R. 1967. In memoriam. D. José María Andreu Rubio (1881-1967). 23: 121-125.

396. Compte Sart, Arturo. 1967. Resultados de una 
expedición zoológica a las islas Pitiusas. III Neurópteros y Odonatos. 23: 127-142.

397. Martín, M ${ }^{\mathrm{a}} \mathrm{Paz}$ \& Jiménez Millán, F. 1967. Contribución al conocimiento de las especies de malófagos existentes en España. 23: 143-158.

398. Agenjo, R. 1967. La verdadera identidad taxonómica de la falena conocida como "rosquilla negra", muy dañina plaga de nuestros cultivos hortícolas, a quien corresponde el nombre científico de Spodoptera littoralis (B., 1833) (Lep. Noctuidae). 23: 159-170, lámina V.

399. Isart, Juan. 1967. El lixus de las habas, Lixus algirus (L.) (Col., Curculionidae). 23: 171-204, láminas VI-VII.

400. Isart, Juan. 1967. Ichneumon sarcitorius L. parásito del lepidóptero Hydraecia xanthenes (Germ.) (Hym. Ichneumonidae). 23: 205-206.

401. Agenjo, R. 1967. Lycia hirtaria (Cl., 1759), geómetra no señalada todavía de los chopos españoles (Lep. Geometridae). 23: 207-214, lámina VIII.

402. Templado, Joaquín. 1967. La distribución geográfica de Dicranura iberica Templ. y Ort. (Lep. Notodontidae). 23: 215-219.

403. Gómez de Aizpúrua, Carlos \& Rupérez, Adolfo. 1967. Epizootia espontánea sobre Thaumetopoea pityocampa Schiff., causada por un virus citoplásmico, Smithiavirus pityocampae Vag. 23: 221-226.

404. Agenjo, R. 1967. La fábula de la cigarra y la hormiga. 23: 227-233, lámina IX

405. Agenjo, R. 1967. Catálogo ordenador de los lepidópteros de España. Gelechiidae, Holcopogonidae, Carposinidae, Tortricidae, Cochylidae, Acentropidae, Cossidae, Limacodidae, Epipyropidae, Zygaenidae, Thyrididae, Drepanidae, Thylatiridae (= Cymatophoridae). 23: sin paginar.

406. Agenjo, R. 1968. Los buenos caracteres definidores de la especie y de las otras categorías sistemáticas superiores en Lepidoptera. 24: 003-024, láminas I-IV.

407. Agenjo, R. 1968. Impresiones de un viaje a Rusia con motivo del XIII Congreso Internacional de Entomología de Moscova. 24: 025-044, lámina V.

408. Agenjo, R. 1968. Tres nuevas razas de la "superespecies" Plebejus (Lysandra) coridon (Poda, 1761) (Lep. Lycaenidae). 24: 045-048.

409. Agenjo, Ramón. 1968. Contribución al conocimiento de la fáunula lepidopterológica ibérica. Seccion de capturas (VI). 24: 049-060.

410. Gómez Bustillo, Miguel R. 1968. La península de la Magdalena, en Santander: paraíso lepidopterológico. 24: 061-066, lámina VI.

411. Cobos, A. 1968. Nuevos materiales para el estudio del género Gynandrophthalma Lac. (Col. Chrysomelidae). 24: 067-077.

412. Español, F. 1968. Tenebriónidos de la región SaxSalinas, provincia de Alicante (Coleoptera). 24: 079-086.

413. Español, F. 1968. Los Phaleriinae de la Península Ibérica e Islas Baleares (Col. Tenebrionidae). 24: 087-094

414. Lagar, Angel. 1968. El género Criocephalus
Mulsant en la Península Ibérica y Baleares (Col. Cerambycidae). 24: 095-100.

415. Pardo Alcaide, Anselmo. 1968. Analectas Entomologicas. VIII. Los Malachius Fabricius de la fauna ibérica (Col. Malachiidae). 24: 101-114.

416. Suárez, Francisco J. 1968. Trogaspidia castellana (Mercet, 1903): aclaracion de un error de procedencia y correcciones sinonímicas derivadas de la misma (Hym. Mutillidae). 24: 115-122.

417. Salom, Francisca. 1968. Sobre Haematopota crassicornis Wahlberg (Dipt. Tabanidae). 24: 123-135.

418. Ribes, J. 1968. Notas sobre arádidos ibéricos (Hem. Heteroptera). 24: 137-141.

419. Pérez-Iñigo, Carlos. 1968. Ácaros oribátidos de suelos de España peninsular e Islas Baleares $\left(1^{\mathrm{a}}\right.$ Parte) (Acari, Oribatei). 24: 143-237.

420. Isart, Juan. 1968. Las principales plagas de la remolacha en España y métodos de lucha. 24: 239-252.

421. Rey, José $\mathbf{M}^{\mathrm{a}}$. 1968. Desarrollo de una dieta larvaria para el cultivo de Dacus oleae Gmel. (Dipt. Tripetidae). 24: 253-259.

422. Rey, José $M^{\mathrm{a}}$. 1968. Tendencias actuales en la lucha contra los insectos. 24: 261-279.

423. Templado, J. \& Morillo, C. 1968. Observaciones preliminares sobre la invernación de Dacus oleae en zonas de clima mediterráneo continental (Dipt. Tripetidae). 24: 281-287.

424. Agenjo, Ramón. 1968. Semblanza de Don Juan Mieg, físico, químico y naturalista decimonónico, autor del primer manual de Entomología publicado en España. 24: 289-304, láminas VII-IX.

425. Agenjo, Ramón. 1969. El tomo XXV de "Graellsia,". 25: 003-104.

426. Agenjo, R. 1969. Distribución, bionomía y morfología del "pequeño pavón" Eudia pavonia (L., 1758) en España, con descripción de una nueva subespecie burgalesa, dedicada al Excmo. Sr. D. Alejandro Rodríguez de Valcárcel y Nebreda, presidente de las Cortes Españolas (Lep. Attacidae). 25: 105-118, lámina I.

427. Agenjo, R. 1969. Seis géneros y veinte especies de Noctuidae nuevos para España (Lep.). 25: 119140, láminas II-VI.

428. Agenjo, R. 1969. Sobre la presencia en España de la Colotis evagore nouna Lucas, 1849 (Lep. Pieridae). 25: 141-148, lámina VII.

429. Agenjo, R. 1969. Otra "autoridad" para la acepción de la voz "falena" en el sentido propugnado por mí el año 1966. 25: 149-152.

430. Agenjo, R. 1969. Contribución al conocimiento de la fáunula lepidopterológica ibérica. Sección de capturas (VII). 25: 153-170, lámina VIII.

431. Agenjo, R. 1969. In memoriam. Ilmo. Sr. Dr. D. Pedro Alfaro Arregui (1914-1969). 25: 171-174.

432. Galante Patiño, Eduardo; Galante Patiño, José Antonio \& Galante Patiño, Javier. 1969. Sobre una emigración de "cardera" Vanessa cardui (L., 1758) observada en Jaca, provincia de Huesca, durante el mes de mayo de 1970 (Lep. Nymphalidae). 25: 175-177.

433. Ganzo, J. 1969. Divulgación anticientífica. 25: 
179-182.

434. Cobos, A. 1969. Revisión de los bupréstidos de Canarias. Apéndice II (Coleoptera). 25: 183-186.

435. Español, F. 1969. Notas sobre anóbidos (Coleoptera). 25: 187-194.

436. Ferreira, Raul Nascimento. 1969. Contribuição para um catálogo da família Staphylinidae da fauna portuguesa. V. Os estafilinideos da Pateira de fermentelos. 25: 195-200.

437. Pardo Alcaide, Anselmo. 1969. Analectas entomológicas IX. Los Ebaeus Erichson de la fauna ibérica (Col. Malachiidae). 25: 201-212.

438. Plata, P. 1969. Sobre la presencia en España del género Amphicoma Latreille, 1807 (Col. Scarabaeidae). 25: 213-216.

439. Janvier, H. 1969. Apuntes sobre las Hylaeus F., 1793, de España (Hym. Apidae). 25: 217-227.

440. Mingo, Elvira. 1969. Sobre las Holopyga Dahlb., 1854, de España (Hym. Chrysididae). 25: 229239.

441. Mingo, Elvira. 1969. In Memoriam. Excmo. Sr. Dr. D. José Juan del Junco y Reyes (1890-1970). 25: 241-243

442. Suárez, Francisco J. 1969. Tres nuevos mutílidos españoles (Hym. Mutillidae). 25: 245-259.

443. Fernandes, J. de A. 1969. O "efeito de grupo" nos insectos ortópteros. 25: 261-278.

444. Bonnet, Pierre. 1969. Las arañas. 25: 279-296.

445. Pérez-Iñigo, Carlos. 1969. Los ácaros y la importancia de su estudio. 25: 297-315.

446. Isart, Juan. 1969. El "taladro de la alcachofa", Hydraecia xanthenes (Germ.) y medios para combatirlo (Lep. Noctuidae). 25: 317-324.

447. Robles-Chillida, E. M.; Muñiz, M. \& Blanco Marco, E. 1969. Influencia del ClNa sobre la ultraestructura del corion de Dacus oleae Gmel. (Dipt. Trypetidae). 25: 325-333.

448. Templado, Joaquín. 1969. La selectividad alimentaria de Dicranura iberica Templ. et Ort. (Lep. Notodontidae). 25: 335-338.

449. González Bernáldez, F. 1969. Ecología. 25: 339346.

450. Templado, Joaquín. 1969. Algunos aspectos de mimetismo en los insectos. 25: 347-358, lámina IX.

451. Anónimo. 1969. Anuncio del XIX Congreso Internacional de Entomología. 25: 359.

452. Anónimo. 1969. Lista de suscriptores. 25: 361375 .

453. Agenjo, R. 1970. Las Apatura F., 1807, de España (Lep. Nymphalidae). 26: 003-024, láminas I-II.

454. Agenjo, R. 1970. Nuevas subespecies de ropalóceros ibéricos. 26: 025-036.

455. Gómez Bustillo, Miguel-R. 1970. Hormigas y mariposas. 26: 037-042

456. Lagar Mascaró, Angel. 1970. Coleópteros del delta del río Llobregat. VII Nota. 26: 043-058.

457. Haro Vera, Andrés de. 1970. Los formícidos, grupo de gran interés zoológico (Hym. Formicidae). 26: 059-098.

458. Königsmann, Eberhard. 1970. Himenópteros recogidos en Palamós, provincia de Gerona, por el Profesor Dr. Hans Bischoft. 26: 099-113.

459. Leclercq, Marcel. 1970. Numerosos Tabanidae y Muscidae sobre un caballo en España y descripción de Haematopota enriquei nov. sp. (Dip. Tabanidae). 26: 115-125.

460. Nieto, Juan Manuel. 1970. Pulgón de la Sierra de Béjar, nuevo para la fauna de Europa occidental: Diphyllaphis mordvilkoi (Aizemberg, 1932) (Hem. Aphididae). 26: 127-131.

461. Selga, Dolores. 1970. Catálogo de los colémbolos de la Península Ibérica. 26: 133-283.

462. Sanz, Casilda. 1970. Observaciones sobre la actividad alimenticia de tres especies de isópodos (Isopoda). 26: 285-293.

463. Bonnet, Pierre. 1970. Las arañas $\left(1^{\text {a }}\right.$ Continuación). 26: 295-311.

464. Ramos, P. 1970. Ensayo preliminar de lucha contra el Prays del olivo en Granada (Lep. Yponomeutidae). 26: 313-321.

465. Robles-Chillida, E. M.; Muñiz, M. \& BlancoMarco, E. 1970. Organización submicroscópica del huevo de Dacus oleae Gmel. (Dipt. Trypetidae). 26: 323-330.

466. Templado, Joaquín \& Dorado, E. 1970. La secreción de la glándula yugular en la larva de Dicranura iberica Templ. et Ort. (Lep. Notodontidae). 26: 331-338.

467. Agenjo, R. 1971. Ocho géneros y veinte especies de Geometridae nuevos para España (Lep.). 27: 003-022, láminas I-IV.

468. Agenjo, R. 1971. Contribución al conocimiento de la fáunula lepidopterológica ibérica. Sección de capturas VIII. 27: 023-042, lámina V.

469. Compte, Arturo. 1971. Sobre la cita de Cryptocephalus curvilinea Ol., 1808, de Mallorca (Col. Chrysomelidae). 27: 043-053.

470. Pardo Alcaide, Anselmo. 1971. Analectas Entomológicas. X. Un valiosísimo instrumento para la caza de pequeños insectos: el insufladoraspirador. 27: 055-066.

471. Janvier, Hippolyte. 1971. Una colonia de Argogorytes hispanicus (Merc., 1906) en Málaga (Hym. Sphecidae). 27: 067-077.

472. Ribes, J. 1971. Sobre Piesma rotundata Horváth, 1906 y formas afines (Hem. Piesmidae). 27: 079089.

473. Martín, M. P. 1971. Algunos malófagos parásitos del urogallo en España. 27: 091-101, láminas VI-VII.

474. Simón, J. C. 1971. Estudio de los colémbolos muscícolas de un roquedo de la Sierra de Guadarrama. 27: 103-132.

475. Templado, J. \& Morillo, C. 1971. Focos permanentes de Dacus oleae (Gmel., 1788) en la cuenca del Jarama (Dip. Trypetidae). 27: 133-152.

476. Serrão Nogueira, C. D. 1971. Pragas do pinheiro Acantholyda hieroglyphica (Christ., 1791) (Hym. Pamphiliidae). 27: 153-160.

477. Pérez-Iñigo, Carlos. 1971. Dípteros y coleópteros pseudoparásitos del intestino humano. 27: 161176.

478. Agenjo, R. 1971. In memoriam. Excmo. Sr. Dr. D. 
José del Cañizo Gómez (1894-1972). 27: 177 195.

479. Agenjo, R. 1971. In memoriam. Sir Boris P. Uvarov, K.C.M.G., F.R.S. (1888-1970). 27: $197-$ 200.

480. Agenjo, R. 1972. Localización en Santander del aclimatado arbusto brasileño Araujoia sericifera Brot., asclepiadácea en cuyas fisuras fenestriformes florales quedan apresados los lepidópteros por la espiritrompa cuando acuden a libar (Lepidoptera). 28: 003-014, láminas I-III.

481. Agenjo, R. 1972. Acentropus niveus (Ol., 1791) especie, género y familia nuevos para la fauna portuguesa (Lep., Acentropidae). 28: 015-023.

482. Agenjo, R. 1972. Rectificación. 28: 025-030.

483. Bullón, M. M. 1972. Agdistis pinkeri Bigot, 1972 , de Gran Canaria, nueva sinonimia de Agdistis salsolae Walsingham, 1907, de Tenerife (Lep. Pterophoridae). 28: 031-035.

484. Español, F. 1972. Nuevos datos sobre los Bostrychidae de la fauna española (Col. Cucujoidea). 28: 037-044.

485. Nieto Nafría, Juan-Manuel. 1972. Recopilación de las citas de pulgones (Aphidinea) de España (Hem. Homoptera). 28: 045-102.

486. Bonnet, Pierre. 1972. Las arañas (2 Continuación). 28: 103-122.

487. Rambla, María. 1972. Los opiliones (Arachnida) ( $1^{\mathrm{a}}$ parte). 28: 123-145.

488. Acón, Margarita. 1972. Estudio de una comunidad zooedáfica de un prado de la Sierra de Guadarrama. 28: 147-175.

489. Isart, Juan. 1972. Observaciones bioecológicas sobre Cleonus mendicus (Gyllenhal, 1834) (Col. Curculionidae). 28: 177-201.

490. Selga, Dolores. 1972. El XIV Congreso Internacional de Entomología de Camberra, 1972. 28: 203-207.

491. Agenjo, R. 1973. Pediasia bulloni nova species, descubierta en Bailén (Lep., Crambidae). 29: 003007.

492. Agenjo, R. 1973. Contribución al conocimiento de la fáunula lepidopterológica ibérica. Sección de capturas. (IX). 29: 009-025.

493. Templado, J. \& Ortiz, E. 1973. Nuevos datos sobre Dicranura iberica Templ. et Ort., 1966 (Lep. Notodontidae). 29: 027-036.

494. Vera, R. 1973. Claves de determinación de familias y géneros del orden Trichoptera citados de España peninsular. 29: 037-078

495. Compte, Arturo. 1973. Notas sobre las especies españolas de Aphodius del subgénero Ammoecius (Col. Scarabeidae). 29: 079-094.

496. Español, F. 1973. A propósito de sendas notas del Dr. R. Dajoz sobre los géneros Helioctamenus Schauf. y Ancylopus Cost. (Col. Cucujoidea). 29: 095-098.

497. Plaza, Esperanza. 1973. Acerca de la especie Bulaea lichatschovi (Humm., 1872) (Col. Coccinellidae). 29: 099-110.

498. Yus, Rafael. 1973. Sobre la presencia en la
Península Ibérica del Rhizobius lophantae (Blaisdell, 1892) (Col. Coccinellidae). 29: 111115.

499. Janvier, Hippolyte. 1973. Nidificación de Psenulus concolor (Dahlbom, 1843) (Hymenoptera). 29: 117-142.

500. Leclercq, Marcel. 1973. Misión entomológica a la isla de Tenerife. Estudio de dípteros. 29: 143-152.

501. Fuente, J. A. de la. 1973. Notas sobre la nomenclatura genérica y supragenérica de los coréidos ibéricos. (Hem. Heteroptera). 29: 153-171.

502. Martín, $M^{a}$ Paz. 1973. Malófagos españoles de la familia Gyropidae. 29: 173-182.

503. Bonnet, Pierre. 1973. Las arañas ( $3^{\text {a }}$ Continuación). 29: 183-200.

504. Morillo, Cosme. 1973. El desarrollo de Saissetia oleae (Olivier, 1791) en condiciones controladas (Hem. Coccidae). 29: 201-210.

505. Robles-Chillida, E. M. 1973. Contribución al estudio micromorfológico del huevo y la larva de Ceratitis capitata (Wied., 1824). 29: 211-224, láminas I-III.

506. Abdullah, Mohammad. 1973. Recopilación de noticias sobre insectos comestibles con comentarios personales y recetas culinarias. 29: 225-238.

507. Agenjo, R. 1974. Las Melitaea (Mellicta) deione Gey., 1827-1832, attalia (Rott., 1775) y parthenoides Kef., 1851, en España. (Lep. Nymphalidae). 30: 003-061.

508. Agenjo, R. 1974. Emendata. I. 30: 063-075.

509. Templado, J. 1974. Modalidades de muda en larvas de lepidópteros. I. 30: 077-081.

510. Compte, A. 1974. Un molesto visitante doméstico, el Attagenus megatoma (Fabr., 1798) (Col. Dermestidae). 30: 083-091.

511. Pardo-Alcaide, A. \& Yus, R. 1974. Genera de coleópteros de la Península Ibérica. Familia Silphidae. 30: 093-111.

512. Plaza, E. 1974. Géneros de Nitidulidae de la Península Ibérica (Coleoptera). 30: 113-127.

513. Mingo, E. 1974. Sobre los Euchroreus Latr., 1809, de España (Hym., Chrysididae). 30: 129-142.

514. Isart, Juan. 1974. Distribución geográfica, ciclo biológico y otras observaciones sobre Pegomya hyoscyami (Panzer, 1809) en España (Dipt. Anthomyiidae). 30: 143-162, láminas I-II.

515. Martín, $M^{\text {a }}$ P. 1974. Notas sobre las especies del género Laemobothrion encontradas sobre aves españolas (Malloph. Laemobothriidae). 30: 163172, lámina III.

516. Acón, Margarita. 1974. Colémbolos de musgos corticícolas del Valle de Hecho, Pirineo aragonés. Nota 1. 30: 173-186.

517. Rambla, $M^{\mathrm{a}}$. 1972. Los opiliones (Arachnida) $\left(2^{\mathrm{a}}\right.$ parte). 30: 187-220

518. Morillo, C. 1974. Regulación de las poblaciones de Saissetia oleae (Olivier, 1791). Factores de mortalidad (Hem. Coccidae). 30: 221-231.

519. Isart, J. 1974. La investigación entomológica en la "Rothamsted Experimental Station". 30: 233-243.

520. Anónimo. 1974. Próxima celebración del XV 
Congreso Internacional de Entomología de Washington, U.S.A. (19 al 27 de agosto de 1976). 30: 245-246.

521. Agenjo, Ramón. 1974. Un libro interesante para los entomólogos: "Historia de las teorías evolucionistas". 30: 247-248.

522. Agenjo, R. 1975. Homenaje de Graellsia al Profesor D. Francisco Español, director del Museo de Zoología de Barcelona. 31: 003-031.

523. Agenjo, R. 1975. Tres nuevas subespecies españolas de Coscinia cribaria (L., 1758) (Lep. Arctiidae). 31: 033-048, lám 1.

524. Agenjo, R. 1975. Sobre la tesis doctoral de D. José Amador de la Calle: "Los Noctuidae españoles. Fenología de los Noctuidae del sur de Madrid y norte de Toledo". (Lepidoptera, Heterocera). 31: 049-059.

525. Palanca, Antonio. 1975. Lepidópteros y tiempo atmosférico. 31: 061-077.

526. Templado, J. 1975. Sobre la variación geográfica de los ropalóceros Ibéricos (Lepidoptera). 31: 079-092.

527. Bach, C. 1975. Contribución al conocimiento de las especies ibéricas del género Rhizotrogus Berthold, 1827. (Col. Scarabaeidae). 31: 093-117.

528. Bellés, X. 1975. Notas sobre Speocharis minos Jeannel, 1909 y otros catópidos recogidos en cuevas de la provincia de Burgos (Col. Catopidae). 31: 119-124.

529. Soler, A. G. \& Montes del Olmo, C. 1975. Datos sobre los coleópteros acuáticos de las Islas Baleares (Haliplidae, Dytiscidae, Gyrinidae). I. Ibiza, río de Santa Eulalia. 31: 125-135.

530. Vives Durán, J. \& Vives Noguera, E. 1975. Dos nuevos carábidos ibéricos (Col. Carabidae). 31: 137-141.

531. Kardás, S. J. \& Arozarena, R. 1975. Notas sobre el género Cerceris Latr., 1802 en Canarias (Hym. Sphecidae). 31: 143-156, láminas II-III.

532. Prat, N. 1975. Quironómidos de Cataluña (Diptera). 31: 157-185.

533. Monserrat, V. J. 1975. Una nueva variedad de Ascalaphus longicornis L. en el sureste de España (Neuropt. Ascalaphidae). 31: 187-192.

534. Martín, M. P. \& Gállego, J. 1975. Malófagos recogidos sobre aves en Cataluña (Mallophaga). 31: 193-211.

535. Simón, J. C. \& Selga, D. 1975. Colémbolos de suelo de sabinar en la provincia de Segovia. Nota I. 31: 213-230.

536. Mayol, J. 1975. Hallazgo de Triops cancriformis (Bosc., 1801) y Leptestheria dahalacensis (Rüppel, 1837) en la Isla de Mallorca (Crustacea, Notostraca, Conchostraca). 31; 231-233.

537. Armengol, J. 1975. Los crustáceos de las aguas del Valle de Bigas, Barcelona. 31: 235-246.

538. Bonnet, Pierre. 1975. Las arañas ( $4^{\mathrm{a}} \mathrm{y}$ última continuación). 31: 247-265

539. Rambla, M. 1975. Nota sobre dos laniatores de la Península Ibérica e Ibiza (Arach., Opiliones Laniatores, Phalangodidae). 31: 267-275.

540. Muñiz, M. 1975. Técnica para la evaluación de puesta en Ceratitis capitata (Wied., 1824) (Dipt. Trypetidae). 31: 277-292.

541. Argumosa, J. A. de. 1975. Etnolepidopterología. Método inédito de pintura-tatuaje mediante orugas de piéridos. 31: 293-298, lámina IV.

542. Gadea, E. 1975. Sobre el origen y relaciones filogenéticas de los insectos. 31: 299-319.

543. Haro, A. de. 1975. Caracteres etológicos de los insectos. 31: 321-339.

544. Margalef, R. 1975. Comunicación y engaño. Aspectos e implicaciones de la cripsis, advertencia y mimetismo. 31: 341-356.

545. Agenjo, R. 1976. Contribución al conocimiento de la fáunula lepidopterológica ibérica. Sección de capturas. (X). 32: 003-018, lámina I.

546. Agenjo, R. 1976. Emendata II. 32: 019-034, lámina II.

547. Agenjo, R. 1976. Rectificación. 32: 035-038.

548. Callejo, C. 1976. Apuntes para un catálogo lepidopterológico de la provincia de Cáceres. 32: 039-091.

549. Redondo, V. M. 1976. Lepidoptera caesaraugustana. (Capturas de lepidópteros en Zaragoza y su provincia). 32: 093-166.

550. Templado, J. 1976. Una migración otoñal de Colias crocea Geof. en Mandayona, Guadalajara (Lep. Pieridae). 32: 167-169.

551. Plaza, E. 1976. Los Carpophilus Stephens, 1830, de España (Col. Nitidulidae). 32: 171-192.

552. Janvier, H. 1976. Comportamiento de Xylocopa violacea Linneo, 1758 (Hymenoptera). 32: 193214, láminas III-IV.

553. Morillo, C. 1976. Sobre la distribución de Formica nigricans Em., 1909 en la Península Ibérica (Hym. Formicidae). 32: 215-217.

554. Leclercq, M. 1976. Tabánidos españoles de las provincias de Oviedo y Santander (Diptera). 32: 219-226.

555. Gómez-Menor, J. \& Nieto, J. M. 1976. Contribución al conocimiento de los pulgones de España (Hem. Homoptera Aphidoidea). 32: 227-260.

556. Martín, M P. 1976. Designación de lectotipo para Corizus rubricosus Bolivar, 1879 (Hem., Heteroptera, Coreidae). 32: 261-263.

557. Badolato, R. 1976. Caracteres comparativos entre Sesamia nonagrioides (Lef., 1827) y S. cretica Led., 1857, en sus distintas fases de desarrollo (Lep. Noctuidae). 32: 265- 277.

558. Rey, J. M. 1976. Gestión sobre plagas en Entomología. 32: 279-296.

559. Agenjo, R. 1976. El XI Congreso nacional de Entomología celebrado en Portici-Sorrento, 10-15 de mayo de 1976. 32: 297-307.

560. Agenjo, R. 1976. Catálogo ordenador de los lepidópteros de España. Ctenuchidae, Noilidae, Sarrothripidae, Chloephoridae, Arctiidae, Noctuidae, Dilobidae, Lymantriidae, Euplagiidae. 32: sin paginar.

561. Agenjo, R. 1977. Sobre la probable inexistencia en España de la Aegle vespertalis (Hb., 1803), donde estaría sustituida por Aegle verpertinalis Rbr., 1866 (Lep. Noctuidae). 33: 003-012.

562. Bland, K. P. 1977. Microlepidópteros de los 
Pirineos españoles. 33: 013-024.

563. Templado, J. \& Outerelo, R. 1977. Notodóntidos de Moscoso, Pazos de Borbén (Pontevedra) (Lepidoptera). 33: 025-029.

564. Leclercq, Marcel. 1977. Transporte y dispersión de insectos dañinos: Hermetia illucens (L.) (Dipt. Stratiomyidae). 33: 031-035.

565. Prat, N. 1977. Quironómidos de los embalses españoles ( $1^{\mathrm{a}}$ parte) (Diptera). 33: 037-096.

566. Monserrat, V. J. 1977. Estudio de la armadura genital de Chrysopa alarconi Navás, 1915, y su inclusión en el género Anisochrysa (Neuropt. Chrysopidae). 33: 097-101.

567. Monserrat, V. J. 1977. Coniopteryx arcuata Kis, 1965, nueva para la fauna española (Neuropt. Coniopterygidae). 33: 103-106.

568. Español, F. 1977. Nuevas localizaciones de carábidos cavernícolas ibéricos (Col. Adephaga). 33: 107-112.

569. Pérez-Iñigo Mora, C. 1977. Contribución al conocimiento de las especies españolas del género Phytoecia Muls., 1839 (Col. Cerambycidae). 33: 113-142.

570. Plaza, E. 1977. Los Nitidulini de la Península Ibérica (Col. Nitidulidae). 33: 143-169.

571. Plaza, E. 1977. Los Hippodamini de la Penísula Ibérica (Col. Coccinellidae). 33: 171-197.

572. Mingo, E. 1977. Sobre los Omalus Panz., 1804, de España (Hym. Chrysididae). 33: 199-219.

573. Martín-Mateo, M. P. 1977. Primera cita para España de Laemobothrion vulturis (F., 1775) encontrado sobre Gyps fulvus (Habl.) (Malloph. Laemobothriidae). 33: 221-226.

574. Compte Sart, Arturo. 1977. Nueva cita de Diplacodes lefebvrei (Ramb.) en España (Odonata, Libellulidae). 33: 227-236.

575. Bach de Roca, C. 1977. Ampliación de conocimientos sobre los Machilida, a través del estudio de una colección del Instituto Español de Entomología (Thysanura). 33: 237-246.

576. Acón, M. \& Simón, J. C. 1977. Contribución al conocimiento de los colémbolos muscícolas de la Sierra de Gredos. 33: 247-259.

577. Isart, Juan. 1977. Observaciones sobre Euleia heraclei (Linneo, 1758) en España (Dipt. Tephritidae). 33: 261-278, lámina I.

578. Muñiz, M. \& Rey, José $M^{\mathrm{a}} .1977$. Comportamiento de Ceratitis capitata (Wied.) ante el tratamiento con formaldehído en la dieta larvaria (Dipt. Trypetidae). 33: 279-308.

579. Muñiz, M. \& Robles-Chillida, E. M. 1977. Emendata. Consideraciones sobre dos trabajos de los Dres. Muñiz y Robles-Chillida. 33: 309-310.

580. Agenjo, Ramón. 1977. Catálogo ordenador de los lepidópteros de España. Arctiidae (tercera edición). 33: sin paginar.

581. González Rebollar, José Luis. 1978. Observaciones sobre Lithocolletis joviella Constant, minador de las hojas de la encina (Lep. Gracilariidae). 34: 003-015, láminas I-II.

582. Plaza, Esperanza. 1978. Los Coccinellidae del monte de El Pardo (Coleoptera). 34: 017-026.

583. Izquierdo Moya, $M^{\mathrm{a}}$ Isabel. 1978. Sobre la presencia en España de Barylypa pallida (Grav., 1829) (Hymenoptera, Ichneumonidae). 34: 027029.

584. Vergés Serra, F. 1978. Euménidos de Andalucía y Extremadura (Hymenoptera, Eumenidae). 34: 031-044.

585. Havelka, Peter. 1978. Agregaciones de machos de Forcipomyia bipunctata (L., 1766) y su relación con la presencia de hembras (Dipt. Ceratopogonidae). 34: 045-052.

586. Leclercq, Marcel. 1978. Tabanidae (Diptera) de España y Portugal. 34: 053-057.

587. Prat, Narcís. 1978. Quironómidos de los embalses españoles (Diptera) (2 ${ }^{\mathrm{a}}$ Parte). 34: 059-119.

588. Martín Mateo, M. P.; Albalá, F. \& Sánchez Acedo, C. 1978. Malófagos ectoparásitos de aves de la provincia de Zaragoza. 34: 121-145.

589. Acón Remacha, Margarita. 1978. Contribucion al conocimiento de los psocópteros de España peninsular. 34: 147-170.

590. Monserrat, Victor J. 1978. Sobre los neurópteros ibéricos (I) (Neuroptera, Planipennia). 34: 171176.

591. Monserrat, Victor J. 1978. Contribución al conocimiento de los neurópteros de Toledo (Neuroptera, Planipennia). 34: 177-193.

592. Herrera Mesa, L. 1978. Nota sobre los dermápteros de Navarra. 34: 195-203.

593. Subías, L. S. \& Iturrondobeitia, J. C. 1978. Contribución al conocimiento de los oribátidos (Acarida, Oribatida) del País Vasco, III. 34: 205209.

594. Munilla León, Tomás. 1978. Dimorfismo de dos especies de pignogónidos: Ammothella longipes (Hodge) y Achelia echinata Hodge (Ascorhynchomorpha, Ammotheidae). 34: 212-221.

595. Muñiz, Mariano. 1978. Acción del formaldehído sobre sucesivas generaciones de Ceratitis capitata (Wied.) (Diptera, Trypetidae). 34: 223-235.

596. Caamaño, Beatriz \& Muñiz, Mariano. 1978. Relación entre mortalidad y actividad media molal de electrólitos en huevos de Ceratitis capitata (Wied.) (Diptera, Trypetidae). 34: 237-248.

597. Agenjo, R. 1978. In memoriam. Norman Denbigh Riley, C.B.E. (1890-1979). 34: 250-252.

598. Compte, Arturo. 1978. El V Simposio Internacional de Odonatología celebrado en Sainte-Thérèse (Canadá), 5-11 de agosto de 1979. 34: 253-257.

599. Anónimo. 1978. Anuncio del próximo XVI Congreso Internacional de Entomología. 34: 259260.

600. Alonso Zarazaga, M. A. 1979-1980. Clave preliminar de las familias de coleópteros ibéricos. 3536: 003-062.

601. Serrano, J. 1979-1980. Sobre la presencia de Harpalus (Actephilus) albanicus y Cymindis (Menas) bedeli en la Península Ibérica (Col., Caraboidea). 35-36: 063-067.

602. Gamarra, Purificación. 1979-1980. Claves de 
géneros de la familia Reduviidae de la región Paleártica occidental (Hemiptera - Heteroptera). 35-36: 069-084.

603. Plaza Infante, Esperanza. 1979-1980. Morfología y nomenclatura de la genitalia de los Coccinellidae (coleópteros). 35-36: 085-101.

604. Muñiz, Mariano \& Gil, A. 1979-1980. Análisis de la respuesta cuantal: Acción conjunta de tóxicos en huevos de Ceratitis capitata Wied. (Dipt.: Trypetidae). 35-36: 103-127.

605. Muñiz, Mariano \& Mora, Rosa de. 1979-1980. Fecundidad y fertilidad de Ceratitis capitata (Wied.) bajo la acción de dosis subesterilizantes de radiación gamma. 35-36: 129-141.

606. G. de Jalón, Diego; G. del Tánago, Marta \& G. de Viedma, Manuel. 1979-1980. Importancia de los insectos en los métodos biológicos para el estudio de la calidad de las aguas: necesidad de su conocimiento taxonómicos. 35-36: 143-148.

607. Hernando Díaz, Blanca M M $^{\mathrm{a}}$. 1981. Claves para la determinación de las especies ibéricas de gérridos (Hemiptera-Heteroptera, Gerridae). 37: 003-006.

608. Gayubo, S. F. 1981. Himenópteros superiores de la Sierra de Béjar: Sphecidae. IV. Crabroninae, Myssoninae y Philanthinae. 37: 007-063.

609. Berzosa, Jacinto. 1981. Tisanópteros de la Sierra de Guadarrama. II. Thripidae Stephens, 1829. ( $1^{\text {a }}$ parte) (Ins. Thysanoptera). 37: 065-076.

610. Burgos, Roberto. 1981. Alteraciones morfológicas en Ceratitis capitata (Wied.) por tratamiento con 5-Fluoruracilo en la dieta larvaria (Dipt. Trypetidae). 37: 077-096.

611. Burgos, Roberto \& Muñiz, Mariano. 1981. Efectos del 5-Fluoruracilo sobre el desarrollo larvario de Ceratitis capitata (Wied.) (Dipt. Trypetidae). 37: 097-121.

612. Pinedo, Concha. 1981. Consideraciones sobre la terminología empleada en la descripción de los cantos de los insectos. 37: 123-137.

613. Pozo, Jesús. 1981. Datos sobre las taxocenosis de colémbolos de un prado litoral. 37: 139-156.

614. Alonso, M. L. \& Herrera, L. 1982. Nueva aportación para el conocimiento de los ortópteros de Navarra. 38: 003-019.

615. Berzosa, Jacinto. 1982. Tisanópteros de la Sierra de Guadarrama. III. Thripidae Stephens, $1829\left(2^{\mathrm{a}}\right.$ Parte) (Ins. Thysanoptera). 38: 021-033.

616. Nieves Aldrey, José Luis. 1982. Nuevos datos sobre las especies de los géneros Cyrtoptyx Delucchi, Caenacis Forster, Cecidostiba Thomson y Hobbya Delucchi asociadas con agallas de cinípidos de los robles (Hymenoptera, Pteromalidae). 38: 035-042.

617. Pérez-Iñigo Mora, Carlos. 1982. Los ápidos de la Sierra de Guadarrama. I. Familias Melittidae y Anthophoridae (Hymenoptera, Apoidea). 38: $043-$ 065 .

618. Monserrat, V. J. 1982. Contribución al conocimiento de los neurópteros de Cáceres (Neur., Planipennia). 38: 067-084

619. García Raso, José Enrique. 1982. Penaeidea y
Caridea de las costas de Málaga (región sur-mediterránea española). 38: 085-115.

620. Barace, J. \& Herrera, L. 1982. Contribución al conocimiento de los quilópodos de Navarra: relación de especies y localidades (Myriapoda, Chilopoda). 38: 117-120.

621. Gayubo, S. F. 1982. Sobre la biología de los esfécidos ibéricos. I. (Hym., Sphecidae). 38: 121-128.

622. Mora, Rosa de \& Muñiz, Mariano. 1982. Comportamiento reproductor de Ceratitis capitata (Wied.) ante el tratamiento con formaldehído y radiación gamma (Dipt. Trypetidae). 38: 129-143.

623. Ocete Rubio, Elvira. 1982. Estudio sobre la fecundidad de las hembras de Spodoptera littoralis (Boisd.) (Lep. Noctuidae) en diferentes condiciones de temperatura y alimentación. 38: 145-153.

624. Muñiz, Mariano \& Burgos, Roberto. 1982. Estudio del comportamiento reproductor de Ceratitis capitata (Wied.) ante el tratamiento con 5-Fluoruracilo y radiación gamma (Dipt. Trypetidae). 38: 155-165.

625. Acosta, F. J.; Morales, M. A.; Zorrilla, J. 1982. Polimorfismo y desgaste en la pieza mandibular de Messor barbarus (Hym., Formicidae). 38: 167-173.

626. Muñiz, Mariano. 1982. Inhibición de la fecundidad y fertilidad de Ceratitis capitata (Wied.) por tratamiento con 5-Fluoruracilo en generaciones sucesivas. 38: 175-182.

627. Rodríguez Rodríguez, J. A.; Sánchez-Covisa, A. \& Guillén Llera, J. L. 1982. Sensibilidad actual de culicinos larvarios. I. Insecticidas clorados. 38: 183-187.

628. Fernández Haeger, J. \& Jordano Barbudo, D. 1982. Observaciones sobre una migración de Cynthia cardui (L.) en la Provincia de Córdoba. 38: 189-192.

629. Viejo Montesinos, José Luis. 1982. Acerca de la influencia humana en las comunidades de mariposas. 38: 193-200.

630. Compte Sart, Arturo \& Caminero Bago, Mercedes. 1982. Las comunidades de coleópteros xilófagos de las encinas de los alrededores de Madrid. 38: 201-217.

631. Serrano, J. 1983. Estudio faunístico de los Caraboidea del Alto Tajo (Coleoptera, Adephaga). 39: 003-030.

632. Gil Collado, J. \& Sahuquillo Herráiz, C. 1983. Claves de identificación de Ceratopogonidae de España Peninsular. I. Subfamilias Forcipomyiinae y Dasyheleinae (Dip., Nematocera). 39: 031-045.

633. Martín Mateo, M. P. 1983. Inventario preliminar de los cóccidos de España. I. Diaspididae. 39: 047-071.

634. Baroni-Urbani, C. 1983. Clave para la determinación de los géneros de hormigas neotropicales. 39: 073-082.

635. Izquierdo, Isabel. 1983. Los géneros españoles de Tersilochinae (Hym., Ichn.). 39: 083-091.

636. Nieves Aldrey, J. L. 1983. Contribución al conocimiento de la fauna de himenópteros inquilinos y parásitos en las agallas de Diplolepis mayri 
(Schlechtendal) y Diplolepis eglanteriae (Hartig) (Hym., Cynipidae). 39: 093-102.

637. Pérez-Iñigo Mora, Carlos. 1983. Los ápidos de la Sierra de Guadarrama. II. Familias Andrenidae y Megachilidae (Hymenoptera, Apoidea). 39: 103126.

638. Berzosa, Jacinto. 1983. Tisanópteros de la Sierra de Guadarrama. IV. Phlaeothripidae Uzel, 1895 (Ins., Thysanoptera). 39: 127-137.

639. G.-Valdecasas, Antonio \& Camacho, Ana Isabel 1983. Arrenurus papillator (Müller, 1776) en la Península Ibérica (Acari, Arrenuridae). 39: 139141.

640. Acosta, F. J. \& Martín Zorrilla, J. V.; Álvarez Cobelas, M. 1983. Estudio experimental del polietismo en hormigas a partir del desgaste dentario. I. Cualitativo. 39: 143-155.

641. Acosta, F. J. \& Martín Zorrilla, J. V. 1983. Estudio experimental del polietismo en hormigas a partir del desgaste dentario. II. Cuantitativo. 39: 157-164.

642. Muñiz, Mariano \& Andrés, Presentación. 1983. Investigaciones básicas para la inclusión de Hansenula anomala como aporte proteico en la dieta larvaria de Ceratitis capitata (Wied.) (Dipt. Trypetidae). 39: 165-174.

643. Templado, Joaquín. 1983. La selectividad alimentaria de los notodóntidos europeos (Lep. Notodontidae). 39: 175-179.

644. Fernández Haeger, J. \& Jordano Barbudo, D. 1983. Datos sobre la presencia de Zizeeria knysna (Triemen, 1862) en la provincia de Córdoba. 39: 181-183.

645. Reyes López, J. L. \& Fernández Haeger, J. 1983. Modificación de rastros químicos en Messor barbarus (Linneo, 1767). 39: 185-192.

646. Martín, J. 1983. Confirmación de la cría de D. plexippus en la Península Ibérica (Lep., Danaidae). 39: 193-194.

647. Bonet Betoret, C. 1984. Siete citas valencianas de Trithemis annulata (Palisot de Beauvais, 1805) (Anisoptera, Libellulidae). 40: 003-006.

648. Lagar, Angel. 1984. Palpicornia del curso superior del río Algars (Tarragona -Teruel) (Coleoptera). 40: 007-017.

649. Plaza, Esperanza. 1984. Contribución al conocimiento de los Coccinellidae españoles. Tribus Coccinellini y Psylloborini. 40: 019-061.

650. Martín Mateo, M. P. 1984. Inventario preliminar de los cóccidos de España. II. Asterolecanidae, Kermococcidae, Coccidae y Aclerdidae. 40: 063079 .

651. Gayubo, S. F. 1984. Contribución al conocimiento de los esfécidos de la provincia de Salamanca. III. Astatinae, Larrinae y Crabroninae (Hym. Sphecidae). 40: 081-097.

652. Mingo, E. \& Gayubo, S. F. 1984. Sphecidae de España. II. Pemphredoninae (Hymenoptera). 40: 099-117.

653. Nieves Aldrey, J. L. 1984. Primeros datos sobre los representantes de la familia Ormyridae en España, con descripción de una nueva especie
(Hym., Chalcidoidea). 40: 119-127.

654. Pérez-Iñigo Mora, Carlos 1984. Los ápidos de la Sierra de Guadarrama. III. Familias Colletidae y Haliciidae (Hymenoptera, Apoidea). 40: 129-157.

655. Agenjo, R. 1984. Paso a la sinonimia de Pseudophilotes abencerragus mattonica GómezBustillo, 1982, y comentarios adicionales (Lep., Lycaenidae). 40: 159-162.

656. Martín Cano, J. 1984. Biología comparada de Lampides boeticus (L.), Syntarucus pirithous (L.) y Polyommatus icarus (Rot.) (Lep., Lycaenidae). 40: 163-193

657. Ocete Rubio, E. 1984. Estudio del ciclo biológico de la Spodoptera littoralis (Boisduval) a diferentes temperaturas (Lep., Noctuidae). 40: 195-206.

658. Acosta, F. J.; Martín Zorrilla, J. \& Serrano Talavera, J. M. 1984. Polietismo temporal latente en Messor barbarus (L.). 40: 207-213.

659. Templado, Joaquín. 1984. Piéridos ibéricos y crucíferas. 40: 215-217.

660. Viejo Montesinos, José Luis. 1984. Los lepidópteros ropalóceros de las agrupaciones de Quercus faginea de la subregión de Madrid. 40: 219-227.

661. Galante, Eduardo 1985. Nuevos datos sobre Anthypna iberica Drioli, 1980, y descripción de la hembra (Col., Scarabaeoidea, Glaphyridae). 41: 003-006.

662. Martín Piera, Fermín. 1985. Los géneros de Melolonthini y las especies ibero-baleares de Amphimallon Berthold, 1827, y Monotropus Erichson, 1848. 41: 007-030.

663. Rahola Fabra, P. 1985. Diferenciación entre Onthophagus similis Scriba y Onthophagus opacicollis Reitter (Col., Scarabaeidae). 41: 031-042.

664. Gil Collado, J. \& Sahuquillo Herráiz, C. 1985. Claves de identificación de Ceratopogonidae de España Peninsular. II. Subfamilia Ceratopogoninae (Dip., Nematocera). 41: 043-063.

665. Prat, N. 1985. Variabilidad morfológica de las poblaciones de Cladotanytarsus mancus (Walker, 1856) de los embalses españoles (Diptera, Chironomidae). 41: 065-088.

666. Martín Mateo, M. P. 1985. Inventario preliminar de los cóccidos de España. III. Pseudococcidae, Ortheziidae y Margarodidae. 41: 089-104.

667. Izquierdo, Isabel \& Rey, Carmen. 1985. Ichneumonidae nuevos para Canarias y nuevas localidades (Hymenoptera, Ichn.). 41: 105-111.

668. Nieves Aldrey, J. L. 1985. La colección de cinípidos gallícolas del Instituto Español de Entomología (Hym., Cynipidae, Cynipinae). 41: 113-124.

669. Pujade Villar, J. \& Nieves Aldrey, J. L. 1985. Una nueva especie de Saphonecrus Dalla Torre y Kieffer del Suroeste de Europa: Saphonecrus barbotini n. sp. (Hym., Cypinidae, Cypininae). 41: 125-129.

670. Stary, P.; Michelena, J. M. \& Meliá, A. 1985. Lysiphlebus testaceipes (Cresson, 1880), un parásito exótico de áfidos y agente de control biológico en España (Hym., Aphidiidae). 41: 131-135.

671. Vergés Serra, F. 1985. Estudio monográfico de los 
Eumenes (Latreille, 1802) de España (Hymenoptera, Eumenidae). 41: 137-152.

672. Clemente Espinosa, $\mathbf{M}^{\mathrm{a}}$. Eulalia; García García, $\mathrm{M}^{\mathrm{a}}$. Dolores \& Presa Asensio, Juan José. 1985. Acridofauna de las dunas de Guardamar del Segura (Alicante) (Orthoptera, Acridoidea). 41: 153-166.

673. Pinedo, $\mathrm{M}^{\mathrm{a}}$. Concepción. 1985. Los Tettigoniidae de la Península Ibérica, España insular y norte de Africa. IV. Subfamilia Saginae Stal., 1874 (Orthoptera). 41: 167-172.

674. Pollo Zorita, Ana María. 1985. Oníscidos de la cuenca alta del río Tajo (Isopoda, Crustacea). 41: 173-189.

675. Pollo Zorita, A. María. 1985. Oníscidos en suelos de bosques quemados de la zona del Alto Tiétar (Avila) (Isopoda, Crustacea). 41: 191-196.

676. Templado, J. \& Viejo Montesinos, J. L. 1985. Ninfálidos y paisaje vegetal en la región de Madrid. 41: 197-202.

677. Bhatnagar, B. S. \& Agarwal, G. P. 1985. Estudios sobre la mariposa Euproctis xanthorrhoea (Kollar), especialmente en lo referente a la relación insecto-planta. I. Manera de alimentarse las larvas sobre Phaseolus lunatus. 41: 203-208.

678. Anónimo. 1986. Aviso a los colaboradores de Graellsia. 42: 002.

679. Martín Piera, F. 1986. Los Rhizotrogus Berthold, 1827, ibero-baleares I. Claves de identificación actualizadas (Col., Scarabaeoidea, Melolonthini). 42: 003-018.

680. Plaza Infante, E. 1986. Clave para la identificación de los géneros y catálogo de las especies españolas peninsulares y baleáricas de Coccinellidae (Coleoptera). 42: 019-045.

681. Tallón, Inmaculada \& Bach, Carmen. 1986. Algunos coleópteros de la Sierra de Cabra (Córdoba). 42: 047-060.

682. Baena, M. \& Vázquez, M. A. 1986. Catálogo preliminar de los heterópteros acuáticos ibéricos (Heteroptera: Nepomorpha, Gerromorpha). 42: 061-089.

683. Docavo, I.; Jiménez, R. \& Tormos, J. 1986. La entomofauna del monte de Porta-Coeli. I Vespidae, Eumenidae (Hym., Diploptera). 42: 091-102.

684. Gayubo, S. F. 1986. Fauna esfecidológica de la provincia de Ciudad Real I. Sphecinae, Pemphredoninae, Astatinae y Larrinae (Hymenoptera, Sphecidae). 42: 103-119.

685. Tormos, J. \& Jiménez Peydró, R. 1986. Esfécidos de la provincia de Valencia. (Hym., Sphecoidea). 42: 121-130.

686. Monserrat, V. J. 1986. Contribución al conocimiento de los neurópteros de Almería (Neur., Planipennia). 42: 131-147.

687. G.-Valdecasas, Antonio \& Camacho, Ana Isabel. 1986. Las hidracnelas leníticas de la Sierra de Guadarrama (Acari, Parasitengona, Hydrachnellae). 42: 149-160.

688. Morano, Eduardo \& Fernández, Miguel Angel.
1986. Contribución al conocimiento de la familia Araneidae Latreille, 1806, de la provincia de Madrid (Arachnida: Araneae). 42: 161-174.

689. García Barros, E. 1986. Descripción de las fases preimaginales y biología de Paidia murina (Hb., 1822). (Lepidoptera, Arctiidae). 42: 175-187.

690. Ocete Rubio, M. E. \& López Sánchez, S. 1986. Datos sobre apareamiento y puesta de Spodoptera littoralis (Boisd.) (Lep. Noctuidae). 42: 189-203.

691. Zunino, Mario \& Palestrini, Claudia. 1986. El comportamiento telefágico de Trypocopris pyrenaeus (Charp.) adulto (Coleoptera, Scarabaeoidea: Geotrupidae). 42: 205-216.

692. Acosta, F. J.; Oñate, M. \& Zorrilla, J. M. 1986. El riesgo como principio conservador del reparto funcional en sociedades de Camponotus cruentatus (Latreille, 1802). 42: 217-232.

693. Fernández Haeger, J. 1986. Capturas de Colotis evagore (Klug, 1829) en el sur de España. 42: 233-235.

694. Viejo Montesinos, J. L. \& Templado, Joaquín. 1986. Los piéridos, satíridos y ninfálidos (Lep.) de la region de Madrid en relacion con las formaciones vegetales. 42: 237-265.

695. Reyes López, Joaquín L. 1986. Variabilidad alar en Messor bouvieri (Bondroit, 1918) (Himenoptera). 42: 267-276.

696. Consejo editorial. 1987. Editorial. 43: 001.

697. Cobos, Antonio. 1987. La coleopterofauna endémica almeriense. 43: 003-017.

698. Ferreras Romero, M. \& Morillo Ortiz, R. 1987. Coleópteros hidrocántaros (Adephaga aquatica) de la cuenca del embalse del río Bembezar (Sierra Morena, Córdoba). 43: 019-028.

699. Lagar, Angel. 1987. Palpicornia del Alto Valle del río Brugent (Gerona) (Coleoptera). 43: 029-037.

700. Serrano, J.; Santos, A. de los \& Mañez, M. 1987. Los Caraboidea de Doñana y zonas adyacentes (Coleoptera). 43: 039-048.

701. Yélamos, Tomás. 1987. Nuevos datos sobre Histeridae de la fauna ibérica. (Coleoptera). 43: 049-052.

702. Martínez Ortega, E. \& Conesa Gallego, E. 1987. Estadíos preimaginales de los flebotomos ibéricos. I. Revisión de la quetotaxia numérica (Dip., Psychod.). 43: 053-060.

703. Vilchez Quero, A.; Casas Jiménez, J. \& Luque Castillo, $\mathrm{M}^{\mathrm{a}}$. 1987. Contribución al conocimiento de los quironómidos (Diptera, Chironomidae) del río Guadalquivir (Sierra de Cazorla). 43: 061-078.

704. Rey del Castillo, Carmen. 1987. Las especies españolas de Banchus Fabricius y Branchopsis Rudow (Hym., Ichneumonidae). 43: 079-086.

705. Martín Mateo, M. P. 1987. Notas sobre malófagos parásitos de aves. Otilipeurus turmalis (Denny, 1842) género y especies nuevos para la fauna ibérica. 43: 087-092.

706. Pinedo, M. C. \& Llorente, V. 1987. Orthopteroidea de la provincia de Castellón, con especial referencia a la marisma de Oropesa. 43: 093-109.

707. Baltanás, A.; Rodríguez, J. \& Valdecasas, A. G. 
1987. Clave preliminar para las ninfas de Plecoptera (Insecta) de la Península Ibérica. 43: 111-125.

708. Pérez-Iñigo, Carlos. 1987. Contribución al conocimiento de los oribátidos (Acari, Oribatei) que viven sobre cítricos (I). 43: 127-137.

709. Urones, Carmen. 1987. Las especies de Chiracanthium C.L. Koch, 1939 (Araneae: Clubionidae) en la Península Ibérica. 43: 139152.

710. García-Raso, J. E. 1987. Contribución al conocimiento de los crustáceos decápodos de los fondos blandos del sur de España. 43: 153-169.

711. Cabello, T. \& Vargas, P. 1987. Influencia de la temperatura en la biología de Trichogramma pintoi Voegele (Hym.: Trichogrammatidae). 43: 169177.

712. Baz Ramos, A. 1987. Abundancia y riqueza de las comunidades forestales de mariposas (Lepidoptera: Rhopalocera) y su relación con la altitud en el Sistema Ibérico meridional. 43: 179-192.

713. Gayubo, S. F.; Torres, F. \& Mingo, E. 1987. Efecto de la presion urbana sobre abejas y avispas (Hymenoptera, Aculeata) en Salamanca. II.: Mutillidae y Chrysididae. 43: 193-204.

714. Peris, S. V. 1987. La invasión de las especies de Chrysomya en América (Dipt. Calliphoridae). 43: 205-210.

715. Serrano, J. M.; Acosta, F. J. \& Alvarez, M. 1987. Estructura de las comunidades de hormigas en eriales mediterraneos según criterios funcionales. 43: 211-223.

716. Aguirre, A.; Arcos, M.; Moyano, F. J. \& Pascual, F. 1987. Tipos adaptativos de morfología mandibular en algunas especies de ortópteros ibéricos. 43: 225-235.

717. Vilchez Quero, A. \& Casas Jiménez, J. J. 1987. Variabilidad morfológica estacional de los adultos machos de Pentapedilum nubens Edwards, 1929 (Diptera, Chironomidae). 43: 237-250.

718. Camacho, A. I. 1988. Comunidades acuáticas subterráneas del norte de la Península Ibérica. 44: 001-017.

719. Gomá, J.; Acosta, F. J.; Fernández Larios, P.; Serrano, J. M. \& Zorrilla, J. M. 1988. La colonización de eriales cerealistas como inferencia sucesional. 44: 019-030.

720. Pérez-Iñigo, C. 1988. Catálogo de los oribátidos (Acari, Oribatei) de la Sierra de Guadarrama. 44: 031-064

721. Pérez-Mellado, V.; Gil, M. J.; Guerrero, F.; Pollo, C.; Rodríguez-Merino, E.; Marco, A.; Lizana, M. 1988. Uso del espacio y del tiempo en Lacerta monticola de la Sierra de Gredos. 44: 065-080.

722. Suárez, F. J. 1988. Mirmósidos de la Península Ibérica (Hymenoptera, Myrmosidae). 44: 081158.

723. Yélamos, T. \& Ferrer, J. de. 1988. Catálogo preliminar de los histéridos de la fauna ibero-balear (Coleoptera, Histeridae). 44: 159-203.

724. Giachino, P. M. \& Salgado, J. Mª 1989. Revisión del género Notidocharis Jeannel, 1956 (Col. Bathysciinae). 45: 001-018.

725. Tinaut, A. 1989. Contribución al estudio de los formícidos de la región del estrecho de Gibraltar y su interés biogeográfico (Hym., Formicidae). 45: 019-029.

726. Vigal, C. R. \& Fandos, P. 1989. Estimación de la edad de los fetos y de los períodos de celo y parto de la cabra montés de Gredos. 45: 031-034.

727. Clemente, M. E.; García, M. D. \& Presa, J. J. 1989. Los Gomphocerinae de la Península Ibérica: I. Stenobothrus Fischer, 1853 y Myrmeleotettix Bolívar, 1914. 45: 035-074.

728. Jordana, R. \& Arbea, J. I. 1989. Redescripción de Pseudachorudina angelieri Cassagnau, 1959 (Collembola: Pseudachorutinae). 45: 075-077.

729. Altonaga, K. 1989. El género Vitrea Fitzinger, 1833 (Pulmonata: Stylommatophora: Zonitidae) en la Península Ibérica. 45: 079-112.

730. Ortea, J. \& Rodríguez, G. 1989. Descripción de una nueva especie de Doto Oken, 1815 (Mollusca: Nudibranchia) de las costas de Málaga, sur de España. 45: 113-116.

731. Jiménez-Guirado, D. 1990. Descripción de Vanderlindia hispanica sp. n. (Nematoda: Tylencholaimidae). 46: 003-005.

732. Mariluis, J. C.; Schnack, J. A.; Muzón, J. \& Spinelli, G. R. 1990. Moscas Calliphoridae y Mesembrinellidae de Puerto Iguazú. Composición específica y ecología (Insecta, Diptera). 46: 007-018.

733. Alonso Zarazaga, M. A. 1990. Revision of the supraspecific taxa in the Palaeartic Apionidae Schoenherr, 1823 (Coleoptera, Curculionoidea). 2. Subfamily Apioninae Schoenherr, 1823. Introduction, keys and descriptions. 46: 019-156.

734. Simon Benito, J. C.; Luciañez Sánchez, M. J. 1990. Scaphaphorura corpesiensis nov. sp. del Macizo de Ayllón, Guadalajara (Collembola: Tullbergiinae). 46: 157-160.

735. Jordana, R. \& Arbea, J. I. 1990. Especies ibéricas de Hypogastrura (Ceratophysella) de seis ojos con descripción de tres nuevas especies (Collembola, Hypogastruridae). 46: 161-173.

736. Monserrat, V. J. 1990. Revisión de la obra de L. Navás, II: El género Micromus Rambur, 1842 (Neuropteroidea, Planipennia: Hemerobiidae). 46: 175-190.

737. Clemente, M. E.; García, M. D. \& Presa, J. J. 1990. Los Gomphocerinae de la Península Ibérica: II. Omocestus Bolívar, 1878 (Insecta, Orthoptera, Caelifera). 46: 191-246.

738. Argüello, J. A. 1990. Dieta de una población cantábrica de Lacerta monticola (Sauria: Lacertidae). 46: 247-252.

739. Argüello, J. A. 1990. Biología reproductiva de Lacerta monticola en una población de la Cordillera Cantábrica (Sauria: Lacertidae). 46: 253-261.

740. Arbea, J. I. \& Luciáñez, M. J. 1991. Una nueva especie de Odontella (Superodontella) Stach, 1949 de la Península Ibérica (Collembola, 
Odontellidae). 47: 001-005.

741. Pérez-Iñigo, C. 1991. Contribución al conocimiento de las especies españolas del género Ceratozetes Berlese, 1908 (Acari, Oribatei). 47: 007-016.

742. San Martín Peral, G. 1991. A new species of Pionosyllis Malmgren, 1867 (Polychaeta: Syllidae: Eusyllinae), from Cuba. 47: 017-020.

743. Luciáñez Sánchez, M. J.; Ruiz Ortega, M. \& Simón Benito, J. C. 1991. Consideraciones acerca del género Paratullbergia Womersley, 1930 (Collembola, Tullbergiinae) con la descripción de P. caroli nov. sp. 47: 021-028.

744. Bikuña, B. G. de; Asensio, R. 1991. Variaciones estacionales en la distribución y abundancia de dos especies de anfípodos (Echinogammarus tarraconensis Pinkster, 1973 y Echinogammarus berilloni Catta, 1978 (Cl. Crustacea) en la cuenca del río Mercadillo (País Vasco): zonación y competencia. 47: 029-041.

745. Tizado Morales, E. J.; Nieto Nafría, J. M. 1991. Aportaciones a la afidiofauna leonesa: novedades para la fauna española de la tribu Macrosiphini (Homoptera, Aphididae). 47: 043-048.

746. Hernández, J. M. 1991. Estudio de los caracteres del huevo en diversos Cerambycidae ibéricos y su interés taxonómico (Coleoptera). 47: 049-059.

747. Montserrat, V. J. 1991. Nuevos datos sobre algunas especies del género Hemerobius L., 1758. (Insecta, Neuroptera: Hemerobiidae). 47: 061070

748. Bellés, X. 1991. Los géneros Kedirinus nov. Sundaptinus nov. y Hanumanus nov. en el archipiélago Indo-australiano y sureste asiático, y nuevos datos sobre el género Maheoptinus Pic (Coleoptera, Ptinidae). 47: 071-096.

749. Arbea, J. I. \& Jordana, R. 1991. Tres nuevas especies de neanúridos en la Península Ibérica (Collembola, Poduromorpha, Neanuridae). 47: 097-103.

750. Rolán, E. 1991. La familia Omalogyridae G.O Sars, 1878 (Mollusca, Gastropoda) en el archipiélago de Cabo Verde. 47: 105-116.

751. Sanz Benito, M. J. \& Gurrea Sanz, P. 1991. Inventario y análisis biogeográfico preliminar de las especies de Curculionoidea (Coleoptera) de Genisteae en las sierras del Sistema Central (Península Ibérica). 47: 117-127.

752. Masaferro, J.; Paggi, A. C. \& Rodrigues Capítulo, A. 1991. Estudio poblacional de los quironómidos (Insecta, Diptera) de la Laguna de Lobos, provincia de Buenos Aires, Argentina. 47: 129-138.

753. Pujade Villar, J. 1991. Nuevas aportaciones al conocimiento de Aulogymnus Förster, 1851 (Hym., Chal., Eulophidae) para la Península Ibérica, con la descripción de una especie nueva, Aulogymnus balani sp. n. 47: 139-154.

754. Ronderos, R. A.; Schnack, J. A. \& Macia, A. 1992. Composición y variación estacional de una taxocenosis de Culicidae del ecotono subtropical pampásico (Insecta, Diptera). 48: 003-008

755. González, A. \& Poncio, G. 1992. Análisis del comportamiento sexual de Achaearanea jequirituba Levi, 1963 (Araneae, Theridiidae). 48: 009018.

756. Carles-Tolrá, M. 1992. New and interesting records of Diptera Acalyptrata from Spain. Part II: Heleomyzidae, Trixoscelididae, Chyromyidae, Curtonotidae, Camillidae, Diastatidae and Campichoetidae. 48: 019-024.

757. Agüera, L.; Parejo, C. \& Muñoz, B. 1992. Fauna malacológica terrestre de la vega de Aranjuez y laguna de Ontígola. 48: 025-033.

758. Ortiz-Sánchez, F. J. \& Aguirre-Segura, A. 1992. Comparación de la eficacia de diferentes alturas en la captura de abejas mediante el empleo de trampas de "Moericke" (Hymenoptera, Apoidea). 48: 035-043.

759. García-Barros, E. 1992. Evidence for geographical variation of egg size and fecundity in a satyrine butterfly, Hipparchia semele (L.) (Lepidoptera, Nymphalidae - Satyrinae). 48: 045-052.

760. Gil-Martín, J.; Subías, L. S. \& Arillo, A. 1992. Oribátidos de Marruecos y Sahara Occidental I: O. inferiores (Acari, Oribatida, Macropylina). 48: 053-063.

761. Parejo, C. \& Muñoz, B. 1992. Suboestophora simplicula, nueva denominación para Gasulliella simplicula (Morelet, 1845) y Suboestophora gasulli (Ortiz de Zárate Rocandio y Ortiz de Zárate López, 1961) (Mollusca: Gastropoda: Pulmonata). 48: 065-069.

762. Lobo, J. M. 1992. Microsucesión de insectos en heces de vacuno: influencia de las condiciones ambientales y relación entre grupos tróficos. 48: 071-085.

763. Luciáñez, M. J. \& Simón, J. C. 1992. Proisotoma juaniae nov. sp., nueva especie de colémbolo (Collembola, Isotomidae) de suelos del centro de España. 48: 087-089.

764. Hernández, J. M.; Ortuño, V. M. 1992. Estudio de la genitalia femenina en Iberodorcadion (Breuning, 1943) y comentarios sobre su valor taxonómico (Coleoptera, Cerambycidae). 48: 091097.

765. Arillo, A. \& Sanz de Bremond, C. 1992. La entomofauna fósil del Mioceno superior de la depresión Ceretana. Himenópteros. 48: 099-107.

766. Ortuño, V. M.; Arribas, O. 1992. El complejo poblacional de Abax pyrenaeus Dejean, 1828 (Col. Caraboidea, Pterostichidae). 48: 109-120.

767. Tinaut, A.; Ruano, F. 1992. Braquipterismo y apterismo en formícidos. Morfología y biometría en las hembras de especies ibéricas de vida libre (Hymenoptera: Formicidae). 48: 121-131.

768. Puente, A. I. \& Prieto, C. E. 1992. La superfamilia Helicoidea (Pulmonata: Stylommatophora) en el norte de la Península Ibérica: corología y sectorización malacogeográfica. 48: 133-169.

769. Nieves Aldrey, J. L. 1992. Revisión de las especies europeas del género Callirhytis Förster (Hymenoptera, Cynipidae). 48: 171-183.

770. Ortea, J. \& Martínez, E. 1992. Descripción de 
una nueva especie del género Carminodoris Bergh, 1889 (Mollusca: Opisthobranchia: Nudibranchia) del piso batial del norte de España. 48: 185-188.

771. Torres-Vila, L. M.; Meco, R. \& Bernao, A. 1992. Detección y distribución de Frankliniella occidentalis Pergande, 1985 (Thysanoptera: Thripidae) en Castilla - La Mancha. 48: 189-191.

772. Alonso Zarazaga, M. A. 1992. Rhadinocybini trib. n. in the Apioninae Schoenherr, 1823 (Coleoptera Apionidae). 48: 193.

773. Iglesias Fuente, D. J.; Martín Piera, Fermín \& Lobo, Jorge M. 1992. Distribución geográfica de Heptaulacus algarbiensis (Branco \& Baraud, 1984) en la Península Ibérica (Col. Scarabaeoidea). 48: 194.

774. Nieves, José Luis \& Ramos, $\mathrm{M}^{\mathrm{a}}$ Ángeles (eds.). 1993. Editorial. 49: 003.

775. Castillejo, J. \& Rodríguez, T. 1993. Reseñas históricas sobre el género Arion Ferussac, 1819 en Portugal (Gastropoda, Pulmonata, Arionidae). 49: 005-016.

776. Castillejo, J. \& Rodríguez, T. 1993. Las especies del género Arion Ferussac, 1819 en Portugal (Gastropoda, Pulmonata: Arionidae). 49: 017-037.

777. Spinelli, G. R. \& Duret, J. P. 1993. Las especies neotropicales de Clinohelea (Diptera: Ceratopogonidae). 49: 039-050.

778. Luciáñez, M. J. \& Simón, J. C. 1993. Vicarianza y distribución geográfica de Isotomodes bisetosus e I. gredensis (Collembola, Isotomidae) en la Sierra de Gredos (España). 49: 051-056.

779. Aguirre-Segura, A.; Pascual, F. 1993. Observaciones sobre algunas especies de Anuraphidina (Homoptera, Aphididae) halladas en Almería. 49: 057-062.

780. Ortiz-Sánchez, F. J. \& Aguirre-Segura, A. 1993. Efecto del color sobre las capturas de abejas mediante trampas "Moericke" en el sur de España (Hymenoptera, Apoidea). 49: 063-071.

781. Briones, M. J. I. \& Díaz Cosín, D. J. 1993. Eophila cyanea n. sp. (Lumbricidae, Oligochaeta), a new earthworm species from the Iberian Peninsula. 49: 073-076.

782. Parejo, C.; Refoyo, P.; Almodovar, A. \& Muñoz, B. 1993. Contribución al conocimiento de la malacofauna terrestre entre los rios Henares, Jarama y Tajuña en la Comunidad Autónoma de Madrid. 49: 077-085.

783. Mateos, E. 1993. Pseudosinella subilliciens n. sp., una nueva especie de Pseudosinella (Collembola, Entomobryidae) edáfica del noreste ibérico (Catalunya, España). 49: 087-090.

784. Carles-Tolrá, M. 1993. A new species of Chamaepsila Hendel from the Czech Republic, with a key to the bicolor-group species (Diptera, Psilidae). 49: 091-095.

785. Soriano, Oscar; Ramil, J. \& Cobo, F. 1993. Adiciones a la fauna ibérica de quironómidos (Diptera: Chironomidae). 49: 097-103.

786. Araujo, R. 1993. Pisidium henslowanum
(Bivalvia: Sphaeriidae) en la Península Ibérica. 49: 105-113.

787. Sanchiz, B. \& De la Riva, I. 1993. Remarks on the tarsus of Centrolenid frogs (Amphibia, Anura). 49: 115-117.

788. Sánchez-Ortega, A. \& Ropero-Montero, J. M. 1993. Leuctra franzi paenibaetica $\mathrm{n}$. ssp. del sur de la Península Ibérica (Insecta: Plecoptera). 49: 119-121.

789. Alonso Zarazaga, M. A. 1993. Diagnosis preliminar de Ceratapion (Echinostroma) wanati sp. $\mathrm{n}$. (Coleoptera, Apionidae). 49: 123.

790. Ros, Joandomènec. 1993. Reseña bibliográfica de: A. Guerra, Mollusca, Cephalopoda. 49: 125126.

791. Blas, Marina. 1993. Reseña bibliográfica de: F. Español, Coleoptera, Anobiidae. 49: 126-127.

792. Iturrondobeitia, Juan Carlos. 1993. Reseña bibliográfica de: C. Pérez-Iñigo, Acari, Poronota. 49: 127-128.

793. Baixeras, Joaquín \& Domínguez, Martí. 1993. Reseña bibliográfica de: J. M. Vargas, R. Real \& A. Antúnez, Objetivos y métodos biogeográficos. Aplicaciones en Herpetología. 49: 128.

794. Pleguezuelos, Juan M. 1993. Reseña bibliográfica de: S. Bruno \& S. Maugueri, Guía de las serpientes de Europa. 49: 129-130.

795. Pérez-Iñigo, C. 1994. Dos nuevas especies de damaeidos (Acari: Oribatei: Damaeidae) de la Península Ibérica. 50: 003-007.

796. González, M. A. \& Botosaneanu, L. 1994. Revisión de las especies ibéricas y pirenaicas del género Synagapetus (Trichoptera: Glossosomatidae) con la descripción de una nueva especie. 50: 009-019.

797. Vujic, A. 1994. Description of male of species Triglyphus escalerai Gil Collado, 1929 (Diptera: Syrphidae). 50: 021-024.

798. Tizado, E. J. \& Núñez-Pérez, E. 1994. Aphidius (Tremblayia) artemisicola, a new subgenus and species of Aphidiinae from Spain (Hymenoptera: Braconidae). 50: 025-027.

799. Askew, R. R. 1994. Further observations on Chalcididae (Hymenoptera) from Spain, with some nomenclatural changes and the description of a new species. 50: 029-034.

800. Carballo, J. L.; Sánchez-Moyano, J. E. \& GarcíaGómez, J. C. 1994. Esponjas del estrecho de Gibraltar. I. Esponjas córneas. 50: 035-056.

801. Naranjo, S. A. \& García-Gómez, J. C. 1994. Ascidias litorales del estrecho de Gibraltar: nuevas aportaciones faunísticas. 50: 057-069.

802. Tinaut, A.; Ruano, F.; Hidalgo, J. \& Ballesta, M. 1994. Mirmecocenosis del sistema de dunas del paraje natural Punta Entinas - El Sabinar (Almería) (Hymenoptera; Formicidae). Aspectos taxonómicos, funcionales y biogeográficos. 50: 071-084.

803. López-González, P. J. \& García-Gómez, J. C. 1994. Tres actinarios nuevos para la fauna ibérica (Anthozoa, Actiniaria). 50: 085-093.

804. Cárdenas, A. M.; Molina, M. D. \& Heras, A. M. de 
las. 1994. Morfología larvaria de Hadrocarabus lusitanicus Fabricius, 1801 (Col. Carabidae). 50: 095-099.

805. Simón, J. C.; Luciáñez, M. J.; Ruiz, M. \& Martín, J. 1994. Tres nuevas especies de Isotomodes Axelson (Linnaniemi) 1907 (Insecta, Collembola) de la Península Ibérica. 50: 101-108.

806. Monserrat, V. J. 1994. Nuevos datos sobre los coniopterígidos de las regiones Paleártica y Afrotropical (Neuroptera: Coniopterygidae). 50: 109-127.

807. Álvarez, Juan A. 1994. Briozoos de la campaña Fauna I (sur de la Península Ibérica). Parte II: Cheilostomida Ascophorina y Cyclostomida. 50: 129-145.

808. Güemes, P. J.; Ambrosio, L. de; Puente, L. E. \& Dorda, J. 1994. Los peces recogidos en la campaña oceanográfica "Fauna I" (sur de la Península Ibérica). 50: 147-159.

809. De la Riva, Ignacio. 1994. Description of a new species of Telmatobius from Bolivia (Amphibia: Anura: Leptodactylidae). 50: 161-164.

810. Haitlinger, Ryszard. 1994. A larval mite (Acari, Erythraeidae) parasitizing the buprestid beetle (Insecta, Coleoptera) in Spain. 50: 165-166.

811. Azzouz, M. \& Sánchez-Ortega, A. 1994. Primera captura de Leuctra franzi paenibaetica SánchezOrtega y Ropero-Montero, 1993 (Insecta, Plecoptera: Leuctridae) en el norte de Africa. 50: 167.

812. Carles-Tolrá, M. \& Tschorsnig, H. P. 1994. Nuevos datos sobre taquínidos de la Península Ibérica (Diptera, Tachinidae). 50: 168-169.

813. Giribet de Sebastián, G. \& Carranza Gil-Dolz del
Castellar, S. 1994. Dos nuevas localidades para un nemertino de agua dulce del género Prostoma Dugès, 1828 (Hoplonemertini: Tetrastemmatidae) en la Península Ibérica. 50: 170-172.

814. Villar, J. L. \& Barranco, P. 1994. Pseudoyersinia paui (Bolívar, 1898). Un nuevo mántido para la fauna de Andalucía (Mantodea, Amelinae). 50: 173.

815. Velázquez de Castro, A. J. \& Outerelo, R. 1994. Primera cita de Styphloderes exsculptus (Boheman, 1843) para la Península Ibérica (Coleoptera, Curculionidae: Molytinae). 50: 174-175.

816. Alonso Zarazaga, M. A. \& López-Colón, J. I. 1994. Designación de neotipo y sustitución de un nombre específico homónimo en el género Geotrupes Latreille (Coleoptera, Geotrupidae). 50: 177.

817. Alonso Zarazaga, M. A. \& Yélamos, T. 1994. Modificaciones al catálogo mundial de Histeridae de S. Mazur (Coleoptera). 50: 178-179.

818. Alonso Zarazaga, M. A. \& Vázquez, X. A. 1994. Addenda y corrigenda a la nomenclatura de diversos táxones de Oedemeridae y Pyrochroidae (Coleoptera) en el volumen 5 de Fauna Ibérica. 50: 180 .

819. Edmonds, S. J. 1994. Reseña bibliográfica de: J.L. Saiz Salinas,. Sipuncula. 50: 181.

820. Fernández Gayubo, Severiano. 1994. Reseña bibliográfica de: E. Mingo, Hymenoptera, Chrysididae. 50: 181-182.

821. Petitpierre, Eduard. 1994. Reseña bibliográfica de: X. Vazquez, Coleoptera. Oedemeridae, Pyrochroidae, Pythidae, Mycteridae. 50: 182-183. 


\section{ÍNDICE DE AUTORES}

Se ha intentado averiguar, dentro de lo posible, el nombre completo y dos apellidos de los autores españoles de Graellsia. Para ello se ha utilizado la recopilación de Martín Albaladejo (1994) y la base de datos del Proyecto "Fauna Ibérica", aunque no se ha conseguido encontrar referencias en todos los casos.

Consejo Editorial: 696, 774

Instituto Español de Entomología: 212

Anónimo: 5, 9, 13, 17, 20, 31, 44, 49, 65, 69, 135, 137, $144,167,188,208,244,245,246,247,253,254$, $255,256,285,286,287,288,294,295,296,297$, $312,313,327,328,329,330,331,350,351,352$, $353,354,355,356,368,369,379,380,381,451$, $452,520,599,678$

Abdullah, Mohammad: 506

Acón Remacha, Margarita: 488, 516, 576, 589

Acosta Salmerón, Francisco Javier: 625, 640, 641, $658,692,715,719$

Agarwal, G.P.: 677

Agenjo Cecilia, Casilda: 154, 182

Agenjo Cecilia, Ramón: 2, 30, 55, 76, 80, 86, 90, 95, $98,101,104,111,130,132,136,143,149,150,152$, $155,157,158,164,168,169,170,181,187,189$, $190,191,197,198,209,210,211,213,224,228$, $234,235,236,273,298,299,314,315,316,332$, $333,334,357,370,371,382,383,384,385,386$, $387,388,395,398,401,404,405,406,407,408$, $409,424,425,426,427,428,429,430,431,453$, $454,467,468,478,479,480,481,482,491,492$, $507,508,521,522,523,524,545,546,547,559$, $560,561,580,597,655$

Agüera, L.: 757

Aguirre Segura, Antonio: 716, 758, 779, 780

Albalá Pérez, Francisco: 588

Alfaro Arregui, Pedro: 153

Almodovar, A.: 782

Alonso Ramos, María Luz: 614

Alonso Zarazaga, Miguel Ángel: 600, 733, 772, 789, $816,817,818$

Altonaga Sustecha, Kepa: 729

Álvarez Cobelas, Miguel: 640, 715

Álvarez Sánchez, Julio: 10, 26

Álvarez Fernández, Juan Antonio: 807

Alvim, Henrique de Sousa: 375

Ambrosio Blázquez, Luis de: 808

Andrés, María Presentación: 642

Anduiza, Rafael: 126

Araujo Armero, Rafael: 786

Arbea Polite, Javier I.: 728, 735, 740, 749

Arcos Dueñas, Marina: 716

Argüello, J.A.: 738, 739

Argumosa Valdés, José Angel de: 541

Arillo Aranda, Antonio: 760, 765

Armengol Bachero, Juan: 537

Arozarena Doblado, Rafael: 317, 364, 531

Arribas Amo, Óscar: 766

Arroyo Varela, Manuel: 348

Asensio, R.: 744

Askew, R.R.: 799

Aubert, J.: 282
Azevedo Silva, Francisco: 347

Azzouz, Malika: 811

Bach Piella, Carmen: 527,575, 681

Badolato, Rafael: 557

Baena Ruiz, Manuel: 682

Báguena Corella, Luis: 163, 179, 202, 214, 237, 248, 257, 258, 259, 266, 267, 290, 338

Baixeras Almela, Joaquín: 793

Balcells Rocamora, Enrique: 307

Baltanás Gentil, José Ángel: 707

Ballesta Franco, Miguel: 802

Barace, J.: 620

Baraud, Jacques: 389

Baroni-Urbani, C.: 634

Barranco Vega, Pablo: 814

Baz Ramos, Arturo: 712

Bellés Ros, Xavier: 528, 748

Benítez Morera, Antonio: 15, 41, 59, 73, 106, 205

Bernao, A.: 771

Berzosa Durán, Jacinto: 609, 615, 638

Bhatnagar, B.S.: 677

Bikuña, B. G. de: 744

Blanco Marco, E.: 447, 465

Bland, K.P.: 562

Blas Esteban, Marina: 791

Bonet Betoret, Carlos: 647

Bonnet, Pierre: 444, 463, 486, 503, 538

Botosaneanu, Lazare: 796

Breuning, Stephan: 290

Briones, M.J. I.: véase Iglesias Briones, María Jesús

Bullón Ramírez, Agustín: 134

Bullón Sopelana, María Manuela: 483

Burgos, Roberto: 610, 611, 624

Caamaño, Beatriz: 596

Cabello García, Tomás: 711

Cabral, M.T.E. Cardoso: 376

Cadahía Cicuéndez, Domingo: 225

Callejo Serrano, Carlos: 548

Camacho Pérez, Ana Isabel: 639, 687, 718

Caminero Bago, Mercedes: 630

Cañizo Gómez, José del: 6, 54, 60, 85, 226

Capilla Caballero, Juan: 292

Carballo, J.L.: 800

Cárdenas Talaverón, Ana María: 804

Carles-Tolrá Hjorth-Andersen, Miguel: 756, 784, 812

Carmona, María Manuela: 346, 375

Carranza Gil-Dolz del Castellar, S.: 813

Casas Jiménez, José Jesús: 703, 717

Castillejo Murillo, José: 775, 776

Castro Guillermín, Luis de: 79, 83, 94

Ceballos Fernández de Córdoba, Gonzalo: 1, 18, 39 , $50,57,66,70,99,105,129,165,175,206,263,272$, 274, 302, 310, 335, 336, 372

Ceballos Jiménez, Pedro: 249, 303

Clemente Espinosa, $\mathbf{M}^{\mathbf{a}}$ Eulalia: 672, 727, 737 
Cobo Gradín, Fernando: 785

Cobos Sánchez, Antonio: 115, 219, 238, 276, 339, 340, 411, 434, 697

Codina Padilla, Francisco: 221, 260, 277, 278, 279, 304, 305

Compte Sart, Arturo: 118, 250, 264, 306, 318, 396, 469, 495, 510, 574, 598, 630

Conesa Gallego, Encarnación: 702

De la Riva De la Viña, Ignacio: 787, 809

Díaz Cosín, Darío J.: 781

Dicenta Ballester, Antonio: 307

Diniz, Manuel de Assunção: 289, 359

Docavo Alberti, Ignacio: 52, 193, 227, 233, 243, 252 $265,271,284,293,311,326,337,349,367,378,683$

Domínguez Romero, Martí: 793

Dorado Bernal, Eloy: 466

Dorda Dorda, Jesús: 808

Duret, J.P.: 777

Ebner, R.: 242

Edmonds, S.J.: 819

Español Coll, Francisco: 3, 27, 36, 67, 75, 82, 87, 100, $103,110,114,119,120,139,145,146,147,148$ $159,160,172,174,185,199,217,230,239,240$, $261,268,280,291,308,322,323,324,341,360$, $373,390,412,413,435,484,496,568$

Fandos París, Paulino: 726

Feliu Quadreny, Sebastián: 35

Fernandes, J. de Almeida: 344, 443

Fernández Gayubo, Severiano: 608, 621，651，652, $684,713,820$

Fernández Haeger, Juan: 628, 644, 645, 693

Fernández Larios, P.: 719

Fernández López, José María: 72, 78, 97, 107, 109, $116,128,140,162,177$

Fernández Porter, Carlos: 38

Ferrández Peñalver, Miguel Ángel: 688

Ferreira, Raúl do Nascimento: 361, 436

Ferrer Andreu, Juan de: 723

Ferreras Romero, Manuel: 698

Flores Casas, Hilario: 61, 91, 133, 269, 283

Fuente Freyre, José Antonio de la: 501

Gadea Buisán, Enrique: 542

Galante Patiño, Eduardo: 432, 661

Galante Patiño, Javier: 432

Galante Patiño, José Antonio: 432

Gállego Berenguer, Jaime: 534

Gamarra Hidalgo, Purificación: 602

Ganzo Mediavilla, Jesús: 433

García Barros Saura, Enrique: 689, 759

García de Jalón Lastra, Diego: 606

García de Viedma Hitos, Manuel Rafael: 275, 321, 343, 606

García García, María Dolores: 672, 727, 737

García Gómez, José Carlos: 800, 801, 803

García Raso, José Enrique: 619, 710

García-Valdecasas Huelin, Antonio: 639, 687, 707

Gayubo, S.F.: véase Fernández Gayubo

Giachino, P.M.: 724

Gil Collado, Juan: 632, 664

Gil Criado, Ángel: 604

Gil Martín, Jesús: 760
Gil, M.J.: 721

Giribet de Sebastián, G.: 813

Gomá, Jaime: 719

Gómez Bustillo, Miguel Ricardo: 410, 455

Gómez de Aizpúrua, Carlos: 403

Gómez Ruano, Ramiro: 301

Gómez-Menor Guerrero, Juan Miguel: 215, 216

Gómez-Menor Ortega, Juan: 131, 151, 176, 232, 251, 555

González Alda, A.: 755

González Bernáldez, Fernando: 449

González del Tánago, Marta: 606

González González, Marcos Antonio: 796

González López, Francisco: 358

González Rebollar, José Luis: 581

Güemes Chozas, Pedro José: 808

Guerrero, F.: 721

Guillén Llera, José Luis: 627

Gurrea Sanz, María Pilar: 751

Haitlinger, Ryszard: 810

Haro Vera, Andrés de: 457, 543

Harz, Kurt: 366

Havelka, Peter: 585

Heath, J.: 229

Heras, A.M. de las: 804

Hernández De Miguel, José María: 746, 764

Hernando Díaz, Blanca Ma' 607

Herrera Mesa, Luis: 592, 614, 620

Hidalgo Naverrete, José: 802

Ibarra Montis, Mariana de: 23

Iglesias Briones, María Jesús: 781

Iglesias Fuente, Domingo José: 773

Isart Sabí, Juan: 399, 400, 420, 446, 489, 514, 519, 577

Iturrondobeitia Bilbao, Juan Carlos: 593, 792

Izquierdo Moya, María Isabel: 583, 635, 667

Janvier, Hippolyte: 439, 471, 499, 552

Jiménez Millán, Fernando: 397

Jiménez Peydró, Ricardo: 683, 685

Jiménez-Guirado, Domingo: 731

Jordana Butticaz, Rafael: 728, 735, 749

Jordano Barbudo, D.: 628, 644

Kardás, S.J.: 531

Königsmann, Eberhard: 458

Lagar Mascaró, Angel: 48, 77, 117, 122, 161, 173, 184, $186,220,281,325,342,362,391,414,456,648,699$

Lajonquière, Yves de: 300

Leclercq, Marcel: 309, 459, 500, 554, 564, 586

Lizana Hevia, Miguel: 721

Lobo, Jorge Juan Miguel: véase Miguel Lobo, Jorge Juan

López Colón, José Ignacio: 816

López González, Pablo José: 803

López Sánchez, Sebastián: 690

Lucas, J.A.W.: 394

Luciáñez Sánchez, María José: 734, 740, 743, 763, 778,805

Luque Castillo, $\mathbf{M}^{\mathrm{a}}$ C.: 703

Llopis Mínguez, Baltasar: 319

Llorente Del Moral, Vicenta: 706

Macia, A.: 754

Mañez Rodríguez, Manuel: 700 
Marco, A.: 721

Margalef López, Ramón: 11, 24, 32, 42, 46, 544

Mariluis, J.C.: 732

Martín Cano, José: 646, 656

Martín Mateo, María Paz: 397, 473, 502, 515, 534, $556,573,588,633,650,666,705$

Martín Piera, Fermín: 662, 679, 773

Martín Zorrilla, J. V.: 625, 640, 641, 658, 692, 719

Martín, J.: 805

Martínez Cueto-Felgueroso, Eugenia María: 770

Martínez Ortega, Ezequiel: 702

Masaferro, J.: 752

Mateos Frías, Eduardo: 783

Mateu Sanpere, Joaquín: 62, 96, 171

Mayol, J.: 536

Meco, R.: 771

Meliá Masiá, Antonio: 670

Michelena Saval, José Manuel: 670

Miguel Lobo, Jorge Juan: 762, 773

Mingo Pérez, Elvira: 365, 392, 440, 441, 513, 572, 652 , 713

Molina, M.D.: 804

Monserrat Montoya, Victor José: 533, 566, 567, 590, $591,618,686,736,747,806$

Montada Brunet, Juan: 88, 108

Montero Cosano, Manuel: 84, 123

Montes del Olmo, Carlos: 529

Mora, Rosa de: 605, 622

Morales Agacino, Eugenio: 178, 377

Morales Bastos, María A.: 625

Morales Morales, E.: 203

Morano Hernández, Eduardo: 688

Morillo Fernández, Cosme: 423, 475, 504, 518, 553

Morillo Ortiz, Rafael: 698

Moyano López, Francisco Javier: 716

Munilla León, Tomás: 594

Muñiz Daza, Mariano: 447, 465, 540, 578, 579, 595, $596,604,605,611,622,624,626,642$

Muñoz, B.: 757, 761, 782

Muzón, J.: 732

Nájera Angulo, Luis: 4, 14, 21, 124

Naranjo Lozano, Santiago A.: 801

Nieto Nafría, Juan Manuel: 460, 485, 555, 745

Nieves Aldrey, José Luis: 616, 636, 653, 668, 669, 769 , 774

Nogueira, C.D. Serrão: 376

Núñez Pérez, Etelvina: 798

Ocete Rubio, María Elvira: 623, 657, 690

Oñate Díaz, Macarena: 692

Orejón, J.M.: 34

Ortea Rato, Jesús Ángel: 730, 770

Ortiz de Vega, Eugenio: 493

Ortiz Sánchez, Francisco Javier: 758, 780

Ortuño Hernández, Vicente M.: 764, 766

Outerelo Domínguez, Raimundo: 563, 815

Paggi, A.C.: 752

Palanca Soler, Antonio: 525

Palau Camps, José María: 7, 47, 63, 92, 121, 125

Palestrini, Claudia: 691

Pardo Alcaide, Anselmo: 28, 40, 43, 53, 138, 183, 200 , 201, 218, 363, 374, 415, 437, 470, 511
Parejo Piñón, Cristina: 757, 761, 782

Pascual Torres, Felipe: 716, 779

Pérez Mellado, Valentín: 721

Pérez-Iñigo Mora, Carlos: 569, 617, 637, 654

Pérez-Iñigo Quintana, Carlos: 419, 445, 477, 708, $720,741,795$

Peris Torres, Salvador Vicente: 204, 223, 231, 262, 714

Petitpierre Vall, Eduard: 821

Pinedo Gurría, Mª Concepción: 612, 673, 706

Plata Negrache, Paulino: 438

Plaza Infante, Esperanza: 497, 512, 551, 570, 571, $582,603,649,680$

Pleguezuelos Gómez, Juan Manuel: 794

Pollo Zorita, Ana María: 674, 675

Pollo, C.: 721

Poncio, G.: 755

Pozo Martínez, Jesús: 613

Prat Fornell, Narcís: 532, 565, 587, 665

Presa Asensio, Juan José: 672, 727, 737

Prieto Sierra, Carlos Enrique: 768

Puente Martínez, Ana Isabel: 768

Puente, Luis Enrique: 808

Pujade Villar, Juli: 669, 753

Pujol Fiol, Manuel: 8, 12, 16, 19

Ragge, D.R.: 345

Rahola Fabra, Pompeu: 663

Rambla Castell, María: 487, 517, 539

Ramil Millarengo, Jacobo: 785

Ramos Clavero, Pedro: 464

Ramos Sánchez, María Angeles: 774

Redondo Veintemillas, Victor Manuel: 549

Refoyo, Pablo: 782

Reglat, Jean-Francois: 194

Rey Arnáiz, José María: 421, 422, 558, 578

Rey del Castillo, Carmen: 667, 704

Reyes López, Joaquín Luis: 645, 695

Ribbe, Carl: 113

Ribes Rius, Jordi: 418, 472

Robles Chillida, Elías Manuel: 447, 465, 505, 579

Rodrigues Capítulo, A.: 752

Rodríguez López, María Teresa: 775, 776

Rodríguez Rodríguez, José Alberto: 627

Rodríguez Vigal, Carlos: 726

Rodríguez, G.: 730

Rodríguez, J.: 707

Rodríguez-Merino, E.: 721

Rolán Mosquera, Emilio: 750

Ronderos, R.A.: 754

Ropero-Montero, José Manuel: 788

Ros Aragonés, Joandomènec: 790

Ruaix, Angelita: 195

Ruano Díaz, Francisca: 767, 802

Ruano, Ramiro G.: véase Gómez Ruano, Ramiro

Ruiz Ortega, M.: 743

Ruiz, M.: 805

Rupérez Cuellar, Adolfo: 166, 270, 403

Sahuquillo Herráiz, César: 632, 664

Sala de Castellarnau, Ignacio: $33,45,71,81,127$

Salgado Costas, José María: 724

Salom Bonet, Francisca: 417 
San Martín Peral, Guillermo: 742

Sánchez Acedo, Caridad: 588

Sánchez-Covisa Villa, Angel: 627

Sánchez-Moyano, J.E.: 800

Sánchez-Ortega, Antonino: 788, 811

Sanchiz Gil de Avalle, Francisco de Borja: 787

Santoro, F.H.: 343

Santos Gómez, Antonio de los: 700

Sanz Benito, María José: 751

Sanz de Bremond, Consuelo: 765

Sanz Gil de Vergara, Casilda: 462

Saz, Eugenio: 58

Schnack, J.A.: 732, 754

Selga Serra, Dolores: 461, 490, 535

Serrano Marino, José: 601, 631, 700

Serrano Talavera, José Manuel: 658, 715, 719

Serrão Nogueira, Carlos David: 347, 476

Simón Benito, José Carlos: 474, 535, 576, 734, 743 $763,778,805$

Smith, C.C.: 229

Smith, Kenneth M.: 192

Soler Andrés, Agustín Ginés: 529

Soriano Hernándo, Oscar: 785

Spinelli, G.R.: 732, 777

Stary, P.: 670

Suárez Egea, Francisco Javier: 275, 416, 442, 722

Subías Esteban, Luis Santos: 593, 760

Tallón, Inmaculada: 681

Templado Castaño, Joaquín: 222, 402, 423, 448, 450, 466, 475, 493, 509, 526, 550, 563, 643, 659, 676, 694

Tinaut Ranera, José Alberto: 725, 767, 802

Tizado Morales, Emilio Jorge: 745, 798

Tormos Ferrando, José: 683, 685
Torres González, Félix: 713

Torres Villa, Luis Miguel: 771

Tschorsnig, H.P.: 812

Urones Jambrina, Carmen: 709

Uvarov, B.P.: 207

Valdecasas, A.G.: véase García-Valdecasas Huelin, Antonio

Van der Godt, V.S.: 394

Varea de Luque, Antonio: 29, 37, 51, 56, 64, 68, 74, 89, 93, 102, 112, 156, 196

Vargas Piqueras, Pedro: 711

Vázquez Martínez, María Ángeles: 682

Vázquez Albalate, Xavier A.: 818

Velázquez de Castro, Antonio Jesús: 815

Vera De la Presentación, Ricardo: 494

Vergés Serra, Francisco: 320, 393, 584, 671

Vidal López, Manuel: 22

Viejo Montesinos, José Luis: 629, 660, 676, 694

Vigal, C.R.: Véase Rodríguez Vigal, Carlos

Vilchez Quero, Antonio: 703, 717

Villar Martínez, José Luis: 814

Vives Durán, Juan: 530

Vives Noguera, Eduardo: 530

Vujic, A.: 797

Winkler, J.R.: 241

Yélamos Gómez, Tomás: 701, 723, 817

Yus Ramos, Rafael: 498, 511

Zarco Segalerva, Eduardo: 141, 142, 180

Zariquiey Álvarez, Ricardo: 25

Zorrilla, J.M.: véase Martín Zorrilla, Juan Vicente.

Zunino, Mario: 691 


\section{NUEVOS TAXONES Y OTROS NOMBRES ZOOLÓGICOS}

Se recopilan los nombres de nuevos taxones, regidos por el Código Internacional de Nomenclatura Zoológica, así como de otras denominaciones zoológicas no sujetas a dicho código. El término "aberración" se refiere casi siempre a morfotipos o a fenotipos discretos, y no tiene connotaciones poblacionales. Por el contrario, especialmente en la literatura antigua, las denominaciones de "forma" y "variedad" son ambiguas. Se ha adoptado el criterio de considerar estas denominaciones como meras variantes infrasubespecíficas, a menos que pudiera deducirse que correspondían a entidades poblacionalmente homogéneas y diferenciables de otras semejantes. En general, y aplicando también el criterio anterior, el término "raza" suele equivaler a subespecie, e incluso se encuentran indistintamente ambas denominaciones en un mismo trabajo (ej. 273). Algunas denominaciones taxómicas nuevas, pero no todas, incluidas en el "Catálogo Ordenador de Lepidópteros de España” de R. Agenjo, pudieran ser nomina nuda según el código de nomenclatura vigente.

Abreviaturas: n.ab.: nueva aberración. n.f.: nueva forma. n.gen.: nuevo género. n.sp.: nueva especie. n.ssp.: nueva subespecie. n.subtrb.: nueva subtribu. n.subgen.: nuevo subgénero. n.trb.: nueva tribu. n.var.: nueva variedad.

\section{NUEVAS FORMAS, VARIEDADES Y ABERRACIONES}

Agrotis ripae velascoi n.f.: 492

Anisoplia baetica deca n.ab: 259

Anisoplia baetica dolens n.ab.: 259

Anisoplia baetica duodeca n.ab.: 259

Anisoplia baetica nona n.ab.: 259

Anisoplia baetica octa n.ab.: 259

Anisoplia baetica prima n.ab.: 259

Anisoplia baetica quarta n.ab.: 259

Anisoplia baetica quatuordeca n.ab: 259

Anisoplia baetica quinquedeca n.ab.: 259

Anisoplia baetica quinta n.ab.: 259

Anisoplia baetica secunda n.ab.: 259

Anisoplia baetica septima n.ab.: 259

Anisoplia baetica sexta n.ab.: 259

Anisoplia baetica tertia n.ab.: 259

Anisoplia baetica tredeca n.ab.: 259

Anisoplia baetica undeca n.ab.: 259

Ascalaphus longicornis montana n.var.: 533

Bulaea lichatschovi pallida maculata n.ab.: 497

Bulaea lichatschovi pallida ornata n.ab.: 497

Bulaea lichatschovi quinquedecimpunctata pseudosuturella n.var.: 497

Coptocephala bistrimaculata bisbimaculata n.ab.: 258

Coptocephala bistrimaculata incorrecta n.ab.: 258

Coptocephala bistrimaculata majoricensis n.ab.: 258

Coptocephala bistrimaculata nudista n.ab.: 258

Coptocephala bistrimaculata peninsularis n.ab.: 258

Coptocephala bistrinotata biloba n.ab.: 258

Coptocephala bistrinotata divisa n.ab.: 258

Coptocephala bistrinotata malacaensis n.ab.: 258

Coptocephala brevicornis extensa n.ab.: 258

Coptocephala fossulata barcinonensis n.ab.: 258

Coptocephala fossulata catalaunica n.ab.: 258

Coscinia cribaria centralis nigrocastanea n.f.: 523

Coscinia cribaria centralis transversa n.f.: 523

Cryptocephalus sexmaculatus ab. simplex n.ab.: 277

Episema grueneri barcoi n.f.: 545

Graellsia isabelae extensa n.f.: 182

Graellsia isabelae rufa n.f.: 182

Graellsia isabelae rufina n.f.: 182

Laccophilus hyalinus bimaculatus n.ab.: 117

Luperus (Calomicrus) setulosus var. subglabrata n.var.: 304

Monotropus stoudingeri octus n.var.: 259

Necrophorus (Necrophorus) vestigator divisa n.ab.: 338

Papilio machaon doloresae n.f.: 468
Plebejus coridon albicans cartujana n.f.: 209

Plebejus (Lysandra) coridon guipuzcoana n.var: 386

Plebejus (Polyommatus) amandus vilarrubiai parva n.f.: 454

Stenoria apicalis catalonica n.ab: 218

Stenoria apicalis kaszabiana n.ab.: 218

Stenoria apicalis luteifrons n.ab.: 218

Stenoria apicalis paucinigra n.ab.: 218

Stizus tridens caesia n.var.: 250

Thestor ballus v. masoi n.f.: 19

Zonites praeusta ramirezi n.ab: 200

Zonitis praeusta gaditana n.ab.: 200

Zonitis praeusta nigriceps n.ab.: 200

\section{NueVAS SUBESPECIES}

Abax pyrenaicus jeannei n.ssp.: 766

Abrostola asclepiades pardoi n.ssp.: 545

Alphasida clementei ziziphi n.ssp.: 697

Alphasida (Glabrasida) gracilis mariae n.ssp.: 697

Alphasida (Glabrasida) gracilis occulta n.ssp.: 697

Alphasida (Glabrasida) parallela almagrerensis n.ssp.: 697

Anthrocera (Lictoria) achillae pardoi n.ssp.: 181

Anthrocera (Silvicola) scabiosae picos n.ssp.: 181

Aphelacarus acarinus sahariensis n.ssp.: 760

Arethusana arethusa albovenata n.ssp.: 454

Asida (Granulasida) zoraida elegans n.ssp.: 697

Asida (Planasida) pygmaea filabrica n.ssp.: 697

Asida (Planasida) pygmaea suarezi n.ssp.: 697

Asida ruficornis kocheri n.ssp.: 339

Brenthis hecate lainezi n.ssp.: 454

Coenonympha dorus cantabrica n.ssp.: 181

Coscinia cribaria centralis n.ssp.: 523

Coscinia cribaria galega n.ssp.: 523

Coscinia cribaria marcosae n. ssp.: 523

Dasylabris iberica valverdei n.ssp.: 442

Dinodes (Iberodinodes) altimirai n.ssp.: 325

Ephippiger ephippiger mischtschenkoi n. ssp.: 366

Ephippiger ephippiger morales-agacinoi n.ssp.: 366

Ephippiger ephippiger vicheti n.ssp.: 366

Eudia pavonia valcarceli n.ssp.: 426

Gynandrophthalma moroderi bacarescensis n.ssp.: 276

Hipparchia alcyone ibarrae n.ssp.: 273

Hipparchia alcyone graellsi n.ssp.: 273

Hipparchia alcyone ochrea n.ssp.: 273

Hipparchia alcyone pardoi n.ssp.: 273

Iolana iolas vareai n.ssp.: 430 
Leuctra franzi paenibaetica n.ssp.: 788

Melitaea desfontainii quevedoi n.ssp.: 430

Olene fascelina iberica n.ssp.: 213

Parnassius apollo pardoi $\mathrm{n} . \mathrm{ssp} .: 454$

Pironia cecilia miegi n.ssp.: 454

Platyderus lusitanicus dertosensis n.ssp.: 325

Plebejus (Agriodaetus) damon acuta n.ssp.: 454

Plebejus (Agriodaetus) damon iris n.ssp.: 454

Plebejus (Agriodaetus) ripartii mozuelica n.ssp.: 454

Plebejus (Lysandra) coridon (albicans) anamariae n.ssp.: 408

Plebejus (Lysandra) coridon (albicans) burgalesa n.sub.: 209

Plebejus (Lysandra) coridon (albicans) esteparina n.sub.: 209

Plebejus (Lysandra) coridon (hispana) gonzalezi n.ssp.: 408

Plebejus (Lysandra) coridon (hispana) semperi n.ssp.: 408

Plebejus (Lysandra) coridon menendez-pelayoi n.ssp.: 209

Plebejus (Lysandra) nivescens estebita n.ssp.: 454

Plebejus (Lysandra) nivescens pascuali n.ssp.: 454

Plebejus (Polyommatus) amandus vilarrubiai n.ssp.: 454

Procris schmidti ariasae n.ssp.: 492

Trichodes crabroniformis hidalgo n.ssp.: 241

Trochantodon tibiellus trichomelas n.ssp.: 374

Xeris spectrum cobosi n.ssp.: 275

Zerynthia rumina armasi n.ssp.: 454

Zerynthia rumina josephignatius n.ssp.: 454

\section{NueVAS ESPECIES}

Ammonicera burnayi n.sp.: 750

Ammonicera multistriata n.sp.: 750

Ammonicera nolai $\mathrm{n} . \mathrm{sp} .:$ 750

Ammonicera oteroi n.sp.: 750

Ammonicera robusta n.sp.: 750

Ammonicera verdensis n.sp.: 750

Amphimallon catalaunicus n.sp.: 202

Aphidius (Tremblayia) artemisicola n.sp.: 798

Atropacarus papillatus n.sp.: 760

Aulogymnus (Olynx) balani n.sp.: 753

Carminodoris spinobranchialis n.sp.: 770

Catalanura florae n.sp.: 749

Ceratapion (Echinostroma) wanati n.sp.: 789

Ceratozetes guadarramicus n.sp.: 741

Chamaepsila martineki n.sp.: 784

Chorebidea dolsi n.sp.: 337

Clinohelea muzoni n.sp.: 777

Coniopteryx (Coniopteryx) greenpeace n.sp.: 806

Coniopteryx (Xeroconiopteryx) pacifista n.sp.: 806

Coniopteryx (Xeroconiopteryx) triantennata n.sp.: 806

Coniopteryx (Xeroconiopteryx) wowifuna n.sp.: 806

Coptocephala arcasi n.sp.: 258

Coptocephala nana n.sp.: 258

Cosmochthonius (Cosmochthonius) maroccanus n.sp.: 760

Damaeus (Damaeus) ortizi n.sp.: 795

Dasylabris canariensis n.sp.: 442

Deutonura deharvengi n.sp.: 749

Doto unguis n.sp.: 730

Eophila cyanea n.sp.: 781

Epichnopterix siederi n.sp.: 382

Epidamaeus subiasi n.sp.: 795

Goniomma compressisquama n.sp.: 802

Gynandrophthalma (Gynandrophthalma) moroderi n.sp.: 411
Gynandrophthalma (Gynandrophthalma) viridipennis n.sp.: 411

Gyrocampa asini n.sp.: 337

Gynandrophthalma (Otiocephala) otini n.sp.: 411

Haematopota enriquei n.sp.: 459

Hanumanus grandicornis n.sp.: 748

Hanumanus Sumateraensis n.sp.: 748

Haplochthonius crassisetosus n.sp.: 760

Haptoderus españoli n.sp.: 530

Helioctamenus fernandezi $\mathrm{n}$.sp.: 340

Heliopathes angustipennis n.sp.: 230

Heliopathes morandi n.sp.: 412

Heliophilus escalerai n.sp.: 3

Hemerobius edui n.sp.: 747

Henicopus (Parahenicopus) españoli n.sp.: 374

Henicopus (Parahenicopus) lagari n.sp.: 374

Heringita heringi n.sp.: 187

Hoplia freyi n.sp.: 389

Hydraena lucasi n.sp.: 648

Hypogastrura deharvengi $\mathrm{n} . \mathrm{sp}: 735$

Hypogastrura jarae n.sp.: 735

Hypogastrura vandalica n.sp.: 735

Isotomodes sotoensis n.sp.: 805

Isotomodes alavensis n.sp.: 805

Isotomodes martae n.sp.: 805

Kedirinus papuanus n.sp.: 748

Leptus mirenae n.sp.: 810

Luperus (Calomicrus) kocheri n.sp.: 279

Luperus (Calomicrus) pardoi n.sp.: 279

Malachius aznaizi n.sp.: 363

Medioppia templadoi n.sp.: 720

Mantura burlinii n.sp.: 277

Micrositus (Eumicrositus) emmanueli n.sp.: 230

Narraga isabel n.sp. 170

Neotrichoppia pseudoconfinis n.sp.: 593

Notidocharis calabrezi n.sp.: 724

Notidocharis meregallii n.sp.: 724

Odontella (Superodontella) carmenae n.sp.: 740

Ormyrus salmantinus n.sp.: 653

Oxybelus dusmeti n.sp.: 365

Papillochthonius astatus n.sp.: 760

Paratullbergia caroli n.sp.: 743

Pediasia bulloni n.sp.: 491

Phaedon (Neophaedon) pseudopyritosus n.sp.: 305

Phylan (Phylan) ribesi n.sp.: 412

Pionosyllis templadoi n.sp.: 742

Platyptilia bigoti n.sp.: 382

Proisotoma juaniae n.sp.: 763

Proutia vareai n.sp.: 382

Pseudosinella españoli n.sp.: 535

Pseudosinella subilliciens n.sp.: 783

Pseudosinella templadoi n.sp.: 535

Psilochalcis frontalis n.sp.: 799

Rhizotrogus carmonensis n.sp.: 259

Rhizotrogus cobosi n.sp.: 202

Rusekella gamae n.sp.: 749

Saphonecrus barbotini n. sp.: 669

Saphonecrus cephaloincisus n.sp.: 668

Scaphaphorura corpesiensis n.sp.: 734

Sellnickochthonius dolosus n.sp.: 760

Spelaeobythus urgellesi n.sp.: 360

Stenopsis pardoi n.sp.: 373 
Stenosis figuerasi n.sp.: 230

Sundaptinus batak n.sp.: 748

Sundaptinus planophthalmus n.sp.: 748

Sundaptinus tamil n.sp.: 748

Sundaptinus wallacei n.sp.: 748

Synagapetus basagureni n.sp.: 796

Tarphius besucheti sp.n.: 280

Telmatobius edaphonastes n.sp.: 809

Tetragonoschema monrosi n.sp.: 115

Tetragonoschema tucumana n.sp.: 115

Trichodromius españoli n.sp.: 530

Vanderlindia hispanica n.sp.: 731

\section{Nuevos SUBGÉNEROS}

Acanephodus (Clementiellus) n. subgen.: 733

Alocentron (Nearctalox) n.subgen.: 733

Aphidius (Tremblayia) n.subgen.: 798

Aspidapion (Koestlinia) n.subgen.: 733

Eilema (Colinia) n.subgen. 580

Eutrichapion (Leconteapion) n.subgen.: 733

Eutrichapion (Phalacrolobus) n.subgen.: 733

Eutrichapion (Psilocalymma) n.subgen.: 733

Hemitrichapion (Dimesomyops) n.subgen.: 733

Hemitrichapion (Lotapion) n.subgen.: 733

Hemitrichapion (Tinocyba) n.subgen.: 733

Holotrichapion (Nesiapion) n.subgen.: 733

Holotrichapion (Schoenius) n.subgen.: 733

Holotrichapion (Apiops) n.subgen.: 733

Lepidapion (Hidryocneme) n.subgen.: 733

Maheoptinus (Kalimantanus) n.subgen.: 748

Melanapion (Rhodapion) n.subgen.: 733

Neapion (Neotropion) n.subgen.: 733

Oreopsyche (Bourgognia) n.subgen. 382

Perapion (Rhaphidoplectron) n.subgen.: 733

Phrissotrichum (Schilskyapion) n.subgen.: 733

Stenopterapion (Cobosiotherium) n.subgen.: 733

\section{NuEVOS GÉNEROS}

Acanephodus n.gen.: 733

Acentrotypus n.gen.: 733

Aizobius n.gen.: 733

Almeria n.gen.: 130

Costaconvexa n.gen.: 130

Fremuthiella n.gen.: 733

Gomezmenoria n.gen.: 382

Hanumanus n.gen.: 748

Heringita n.gen.: 187

Kedirinus n.gen.: 748

Kissingeria n.gen.: 733

Neapion n.gen.: 733

Neotrichoppia n.gen.: 593
Oryxolaemus n.gen.: 733

Osellaeus n.gen.: 733

Papillochthonius n.gen.: 760

Protopirapion n.gen.: 733

Pseudotergumia n.gen.: 104

Ruperezia n.gen.: 405

Spelaeobythus n.gen:. 360

Sundaptinus n.gen.: 748

Wagnerium n.gen.: 733

NUEVAS TRIBUS, SUBTRIBUS Y SUPERTRIBUS

Aspidapiini n.trb.: 733

Aspidapiitae n.supertrb.: 733

Catapiina n.subtrb.: 733

Ceratapiini n.trb.: 733

Exapiini n.trb.: 733

Ixapiini n.trb: 733

Kalcapiini n.trb.: 733

Malvapiini n.trb.: 733

Metapiini n.trb.: 733

Oxystomatina n.subtrb.: 733

Oxystomatini n.trb.: 733

Rhadinocybini n.trb.: 772

Synapiina n.subtrb.: 733

Trichapiina n.subtrb.: 733

NUEVOS NOMBRES POR HOMONIMIA

Brachyunguis gomezmenori nov. nom. [en 485]. Sustituye a Brachyunguis zygophilli Gómez-Menor, 1950, asignado con anterioridad a Brachyunguis zygophilli Newsky, 1928.

Proisotoma najtae nom. nov. [en 461]. Sustituye a Proisotoma inaequalis Selga, 1962, asignado con anterioridad a Proisotoma inaequalis Schäffer, 1898.

En el artículo 360, F. Español menciona 12 taxones a los que asigna notación de nueva especie o subespecie. Estos son: Anillochlamys aurouxi, Anillochlamys urgellesi, Geotrechus (Geotrechidius) seijasi, Geotrechus (Geotrechidius) ubachi, Ildobates neboti, Microtyphlus aurouxi, Spelaeobythus españoli, Spelaeobythus urgellesi, Speonomus aurouxi, Speonomus urgellesi, Speophilus kiesenwetteri andresi, Speophilus subilsi y Speophilus jacasi. Debe entenderse por el contexto, sin embargo, que no hay intención nomenclatural alguna, habiendo sido descritas formalmente en otros lugares por él mismo u otros autores. Concerniente a prioridad nomenclatural, sólo una de ellas (Anillochlamys aurouxi) supone un caso dudoso, al mencionarse la localidad tipo e incluirse una mínima diagnosis diferencial. 


\section{ÍNDICE DE GÉNEROS}

Se ha tratado de incluir todas las denominaciones genéricas aparecidas en la revista, incluso aquellas que figuran únicamente en las listas de sinónimos. Por ello aparecen en ocasiones términos con mínima diferencia de letras y lapsus calami históricos. Algunos errores de esta índole detectados, y atribuibles directamente a la imprenta de Graellsia, no se han indexado siempre. No se incluyen los nombres de géneros que aparecen en los títulos de los trabajos citados sólo en bibliografías, sean éstas de artículos o de necrológicas. Tampoco se incluyen los nombres de subgéneros ni de grupos de especies que aparezcan únicamente como tales, como tampoco los nombres vulgares sin mención del nombre científico latino, o las especies cuyo género aparezca únicamente como abreviatura. Dado el ámbito geográfico tratado en Graellsia, fundamentalmente hispano, los 6.868 nombres genéricos recopilados probablemente suponen una parte considerable de la fauna ibérica, ya que la cantidad de artículos sobre faunas exóticas es muy pequeño.

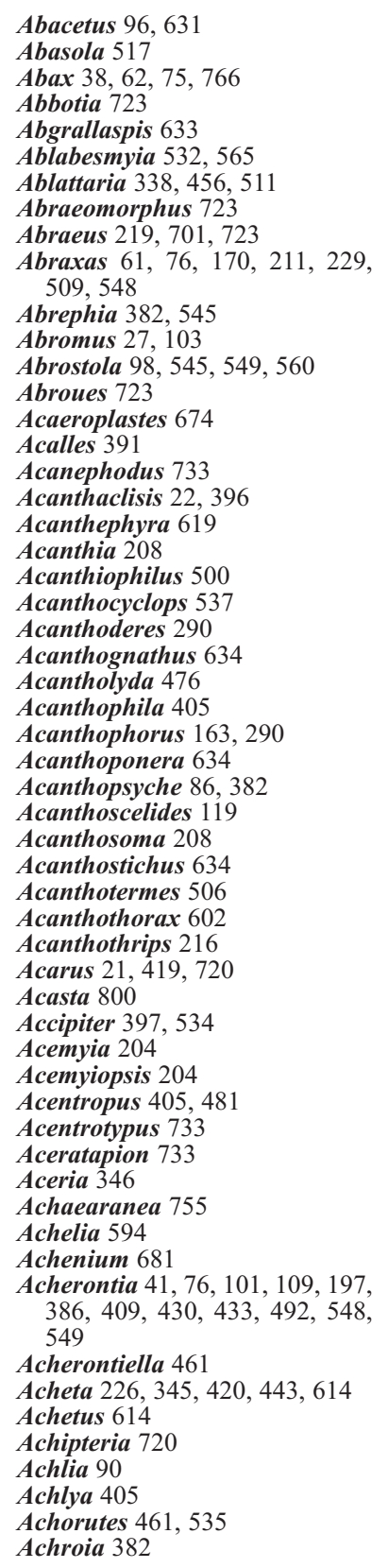

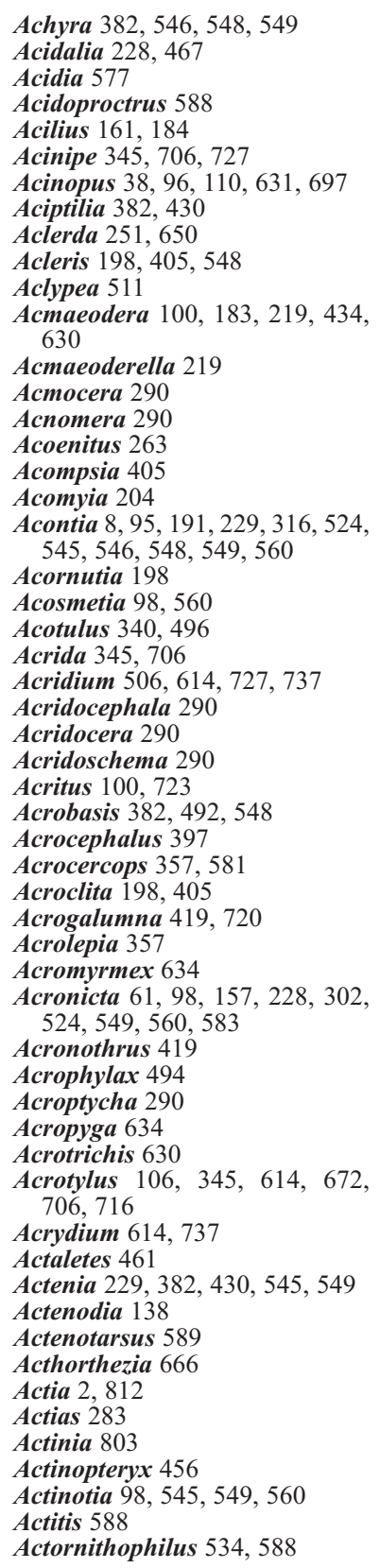

Aculepeira 688
Aculus 346
Acupalpus $38,96,110,391,601$,
$\quad 631,700$
Acylophorus 436
Acyrthosiphon 555,745
Acyrtosiphon 485
Adactylodes $155,170,548,549$
Adalbertia 170,468
Adalia $219,232,456,571,582$,
$\quad 649,680$
Adania 382
Adela $86,357,545,562$
Adelomyrmex 634
Adelops 724
Adelura 193,577
Adephaga 220
Aderpas 290
Adesmia 3,100
Adicella 494
Adiscocaspis 633
Adistemia 87,119
Adleria 668
Adonia $110,140,571,582,680$
Adopaea 61,89
Adopea 19
Adoretus $100,163,506$
Adoristes 720
Adoxophyes 198,405
Adscita $80,90,111,190,194$,
3er 545
Aechmia 370
Aechmites 92
Aedeomyia 754
Aedes $4,11,97,162,543,611$,
$\quad 626,754$
Aedia 98,560
Aegeria $76,86,225,549$
Aegialia 139
Aegle $98,468,549,560,561$
Aegypius 573
Aeletes 723
Aelia $13,125,208$
Aelopus 506
Aenasioidea 251,364
Aepus 110
Aesalus 214
Aeschna 647
Aeshna 396
Aethecerus 336
Aetheocerus 461
Aethes $198,316,405,492,548$
Aethia 98,560
Aethiessa 214
Aethriamantha 264
Affirmaspis 251
Afrotibicina 733
Afrotis 398
Agabiformius 674

Agabus 77, 122, 161, 184, 220, $264,281,391,529,698$

Agalenatea 688

Agapanthia 391, 681, 746 Agapates 358

Agapeta 198, 405, 562

Agapetes 104, 134, 150, 154, 190. 229, 236

Agapetus 494, 796

Agathidium 630

Agculocera 204

Agdistis 210, 314, 382, 406, 430, 468, 483, 492, 545, 559

Agelastica 723

Agelena 26, 444, 538

Ageniaspis 375

Aglais 16, 229, 386, 410, 430, 526, 676, 694

Aglaope 13, 90, 405, 509, 548, 549

Aglenus 103

Aglia 101, 153, 189, 371, 386, 426, 431, 545 Aglossa 179, 228, 382, 430, 492,

Aglycyderes 110, 600

Agnatus 600

Agniolamia 290

Agonia 193

Agonopterix 370

Agonopteryx 316, 386, 406

Agonum 38, 62, 96, 631

Agostenus 96

Agranolamia 290

Agraphopus 501

Agraylea 494

Agrenia 461

Agriades 19, 209

Agrilus 630

Agriope 382, 430

Agriopis 98, 467

Agriotes 391, 420, 446, 681

Agriotis 163

Agriphila 228, 382, 409, 468,

$545,548,549$

Agripon 302

Agrius 492, 545

Agrochola 427, 492, 524, 545, 546,560

Agrocola 548

Agrodiaetus 209, 712

Agromyza 193, 337, 446

Agroperina 98

Agrotera 382

Agrotis 61, 95, 113, 155, 189,

191, 209, 228, 298, 302, 316,

386, 468, 492, 524, 545, 546,

$549,560,711$

Agrypnia 494

Agrypon 302 


\begin{tabular}{|c|c|}
\hline Ahasverus 119 & Amblyopone 634,725 \\
\hline Ahermodontus 28, 259, 495 & Amblyptilia 382 \\
\hline Ailopus 345 & Amblystomus 96 \\
\hline Aiolopus 614, 672, 706, 716 & Amblyteles 127, 129, 263, 274, \\
\hline Airaphilus 100 & $336,372,667$ \\
\hline Aizobius 733 & Amblythrips 615 \\
\hline Akis 47, 92, 100, 119, 341, 412, 810 & Amegilla 289,758 \\
\hline Alabonia 370 & Ameles 345, 706 \\
\hline Alacentron 733 & Amephana 95, 549, 560 \\
\hline Alastor $129,393,584,683$ & Amerobelba 419 \\
\hline Alastorinerus 584 & Amerus 419 \\
\hline Alauda 113 & Ametria 193 \\
\hline Albarracina 98, 213, 409, 560 & Amicta 86 \\
\hline Alca 588 & Amictoides 382, 545 \\
\hline Alcis 546 & Amictoides 316 \\
\hline Alecanopsis 251 & Amimyrmex 634 \\
\hline Alecto 807 & Ammatomus 608 \\
\hline Alectoris 588 & Ammbiota 158 \\
\hline Aleimma 198, 405 & Ammidium 110 \\
\hline Aleochara $110,140,630$ & Ammobates 289, 320, 617 \\
\hline Alepidaspis 365 & Ammobiota 545, 560, 580 \\
\hline Alestrus 110 & Ammobius 100, 120, 240, 341413 \\
\hline Aleucanitis 560 & Amnobius 341 \\
\hline Aleurocanthus 232 & Ammoconia 98, 560 \\
\hline Aleurodamaeus 419, 720 & Ammoecius 259, 495 \\
\hline Aleurolobus 232 & Ammonicera 750 \\
\hline Aleuropteryx 591, 618, 686, 806 & Ammonicerina 750 \\
\hline Aleurotrachelus 232 & Ammophila 250, 531, 543, 621, \\
\hline Aleurotulus 232 & 684,685 \\
\hline Alexeter 372 & Ammoplanellus 652 \\
\hline Aleyrodes 232 & Ammoplanus 81, 652 \\
\hline Algatea 290 & Ammopolia 228, 560 \\
\hline Alispa 382 & Ammothella 594 \\
\hline Allendesalazaria 28,138 & Amnemochthonius 720 \\
\hline Allionix 79 & Amoebe 170 \\
\hline Allochapmania 357 & Amorpha 61 \\
\hline Alloclita 370 & Amorphocephala 600 \\
\hline Allodamaeus 720 & Ampedius 630 \\
\hline Allodynerus 393, 683 & Ampedus 630 \\
\hline Allogalumna 720 & Amphibolus 602 \\
\hline Allogamus 494 & Amphicoma 438 \\
\hline Allogyropus 502 & Amphidesmus 290 \\
\hline Allolobophora 781 & Amphigerontia 589 \\
\hline Allomerus 634 & Amphimallon 202, 214, 220, 527, \\
\hline Allonyx 183 & 662,679 \\
\hline $\begin{array}{l}\text { Allophyes 98, 191, 406, 427, 431, } \\
\quad 524,560\end{array}$ & $\begin{array}{l}\text { Amphinemura } 707 \\
\text { Amphipoea } 427\end{array}$ \\
\hline Allophyton 290 & Amphipyra 98, 427, 524, 549 \\
\hline Allotarsus 219, 374, 697 & Amphisa 545 \\
\hline Allothrombium 445 & Amphithrix 382 \\
\hline Allotrichia 494 & Amphorophora 555 \\
\hline Allozetes 419,720 & Amphotis 512, 570 \\
\hline Alluaudomyia 632, 664 & Amphrophora 485, 499 \\
\hline Almeria 130, 170 & Amphymallon 248 \\
\hline Alocentron 733 & Amydgaloptera 170 \\
\hline Alomya 129 & Amyras 193 \\
\hline Alona 537 & Anabolia 494 \\
\hline Alosimus 138 & Anacaena 529, 648, 699 \\
\hline Alphasida 341, 373, 697 & Anacampsis 405 \\
\hline Alpheus 619,710 & Anachipteria 720 \\
\hline Alphintophagus 47 & Anaciaeschna 396 \\
\hline Alphitobius 47, 120 & Anacridium 204, 345, 672, 706, 716 \\
\hline Alphitophagus 75 & Anadetia 357 \\
\hline Alphitopola 290 & Anagyrus 364 \\
\hline Alsophila 61,170 & Anaitis 61, 154, 170, 211, 228, \\
\hline Alucita 19, 370 & $406,492,548$ \\
\hline Alydus 501 & Anamaspis 633 \\
\hline Alypia 382 & Anaphe 506 \\
\hline Alysia 193 & Anaphothrips 609 \\
\hline Alyson 127, 129 & Anaplectoides 95 \\
\hline Alysson 608 & Anarpia 382 \\
\hline Alytes 744 & Anarsia 405 \\
\hline Amara $38,62,92$ & Anarta 95, 508, 560 \\
\hline Amaroucium 801 & Anas $113,397,588$ \\
\hline Amathes 61, 95, 52 & Anaspis 219 \\
\hline Amaurobius 503, 53 & Anataelia 547 \\
\hline
\end{tabular}

Anaticola 397, 588

Anatis 571, 649, 680

Anatoecus 397, 588

Anatopynia 313

Anatragus 163, 290

Anauxesida 290

Anauxesis 290

Anax 264, 396, 506, 647

Anchinia 370

Anchinoe 770

Anchomenus 631

Anchonemus 96

Anchoscelis 98, 316, 549

Anchotrechus 110

Anchus 96, 631

Anchylis 149

Ancistrocerus 317, 393, 584, 683

Ancylis 198, 405, 562

Ancylocera 204

Ancylodes 382

Ancylolomia 382, 386, 430, 545, 548, 549

Ancylonotus 163, 290, 506

Ancylopus 119, 217, 456, 496

Ancylosis 382

Ancyrosoma 125, 208

Andrena 58, 127, 138, 289, 318 $458,499,617,637,654,758$, 780

Andricus 60, 616, 653, 668, 753, 769

Anechura 242, 592

Anelasmocephalus 517

Anelosimus 486

Anemia 100, 240, 291

Anepia 95

Anerastia 382

Aneuclis 635

Aneurus 418

Aneuxanthis 198, 405

Anexamita 290

Angelia 419, 720

Angerona 170

Angitia 336, 375

Anglais 192

Angosoma 506

Anguila 744

Anguilla 808

Anguitia 263

Angustalius 382, 549

Anilastus 129

Anillochlamys 360, 568

Anisobas 372

Anisochrysa 566, 590, 591, 618

Anisodactylus 38, 62, 96, 220, 631

Anisolabis 344, 345

Anisoplia 186, 214, 259

Anisops 682

Anisopus 477

Anisorhynchus 681

Anisosticta 456, 571, 680

Anisotoma 630

Anmobius 47

Annectocyma 807

Anobium 110, 183, 324, 435 , 456,630

Anochetus 18, 634, 725

Anodonta 786

Anoecia 485, 555

Anogcodes 306, 818

Anomala 88, 139, 163, 186, 214 506

Anomalon 302, 667

Anomalopterygella 494

Anomma 163, 457

Anommatus 63, 103
Anomostherium 666

Anoncodes 306, 818

Anopheles 46, 72, 97, 140, 162, $378,422,754$

Anophia 98, 113, 549

Anoplocephala 419

Anoplocerus 501

Anoplolepis 634

Anoplostetha 290

Anoplotrupes 691

Anoribatella 720

Anoripoda 419

Anospilus 127

Anotiobiella 747

Anoxia 186, 214, 391, 420, 446 662

Anoxus 129

Anoxybelus 365

Anser 588

Antaxius 345

Antepipona 584, 683

Anthaxia 100, 183, 434, 630

Antheraea 283, 333

Antherea 136, 283, 506

Anthia 100

Anthias 808

Anthicus 100, 110, 140, 219, 340,

456, 762

Anthidiellum 289, 637

Anthidium 58, 138, 200, 218, 268, 289, 318, 320, 439, 637, $713,758,780$

Anthidiun 249

Anthocaris 56, 93, 409, 410, 549

Anthocharis 68, 102, 428, 526, 548, 659, 660, 694, 712

Anthocomus 456

Anthocopa 289, 637

Anthocoris 215, 232

Anthomanes 818

Anthometra 170, 228, 386

Anthomyia 179, 365, 514

Anthonomus 44, 611, 626, 751

Anthophila 370, 561

Anthophora 28, 71, 138, 183,

$218,268,289,320,531,617$

Anthores 163

Anthracia 524, 560

Anthracus 96

Anthrax 268, 621

Anthrenus 69, 179, 551, 681

Anthribus 88, 570

Anthrocephalus 799

Anthrocera 76, 80, 90, 154, 157,

181, 189, 191, 316, 508

Anthypna 661

Antichthonidris 634

Antiercta 382

Antigastra 228, 382

Antipa 258, 260, 266, 277

Antisphodrus 360

Antitype 61, 98, 189, 190, 228, 549, 560

Antocharis 12, 89, 113

Antonina 251, 666

Antonogadus 808

Antophila 228

Antrocharidius 360

Antrusa 193, 337

Anua 98, 549

Anuraphis 485, 499, 555, 779

Anurida 461, 535, 613, 749

Anuridella 461

Anurophorus 461

Anybostetha 290

Aonidia 251, 633, 650 
Aonidiella 251, 498, 633

Apaidia 95, 549, 560, 580

Apalus 138, 218

Apamea 427, 524, 545, 546, 560

Apanteles 73, 367, 656, 711

Apantesis 158, 228, 236, 545, 560,580

Apatania 494

Apate 163, 239, 484

Apatele 228, 316, 386, 409, 492 508, 524, 548

Apatema 382

Apatetris 405

Apatura 76, 104, 136, 181, 191

$315,316,371,409,430,453$

482, 526

Apeira 371, 467

Apentates 291

Apericlista 347

Aphaenogaster 39, 634, 715,

719, 723, 725, 767, 802

Aphaenops 568

Aphanarthrum 110, 140

Aphaniosoma 756

Aphanistes 302

Aphanthopus 409

Aphantopus 104, 315

Aphanus 125, 208, 621

Aphelacarus 419, 720, 760

Aphelia 198, 405, 562

Aphelinus 232

Aphelocheirus 682

Aphelopus 336

Apheniastus 290

Aphenogaster 54, 650

Aphidecta 571, 680

Aphidius 84, 232, 798

Aphidura 485

Aphiochaeta 128

Aphis 44, 84, 208, 215, 232, 420

446, 485, 499, 519, 555, 571,

$666,670,745,798$

Aphleps 602

Aphodius 28, 81, 92, 96, 100 139, 186, 219, 237, 477, 495, $600,681,762$

Aphomia 316

Aphonia 382

Aphonura 461

Aphoromma 46

Aphrophora 59

Aphthona 277

Aphycus 364, 518

Apicula 733

Apiochaeta 477

Apiogaster 290

Apiolum 733

Apiomorphus 733

Apion 92, 96, 100, 140, 321, 391. $446,697,733,75$

Apionus 733

Apiotherium 733

Apis 33, 183, 226, 289, 318, 365 ,

$506,531,543,608,617,621$,

$637,654,683,758,780$

Apium 733

Apius 733

Aplasta 61, 170, 548

Aplemonus 733, 772

Aplidium 801

Aploneura 485, 555

Aplota 370

Aplysilla 800

Aplysina 800

Aplysinopsis 800

Apocheima 170, 467
Apochemia 61

Apochina 467

Apoda 86, 157, 191, 405, 468, 508

Apodia 405

Apomecyna 290

Apomempsis 290

Apopestes 61, 98, 113, 302, 409, 430, 548, 549, 560

Aporia 8, 12, 89, 104, 194, 315,

$316,358,468,526,545,548$

$549,659,660,694,712$

Aporodes 228, 316, 382, 549

Aporophyla 61, 98, 409, 524,

$545,548,549,560$

Aporrectodea 781

Apotapion 733, 772

Apotomis 198, 405

Apotomus 96, 700

Appelia 232, 555

Apriona 506

Aproaerema 405

Apterococcus 666

Apterona 86, 168, 382, 562

Apteronympha 600

Apteropeda 304

Apterostigma 634

Apterygida 344

Aptesis 347

Aptinothrips 609

Apus 397

Aquanirmus 588

Aquila 113, 397, 515, 573, 588

Arachnocephalus 345

Aradus 183, 208, 418

Aragonia 405

Araneus 444, 486, 503, 585, 688

Araniella 688

Araschnia 315, 316, 386, 409 430, 526

Araucomyrmex 634

Arboricola 588

Arce 198

Archaeomicromus 736

Archanara 98, 427, 546, 560

Archianthidiellum 637

Archianthidium 289, 637

Archidistoma 801

Archiephestia 382

Archips 198, 380, 405, 545, 549

Archipteria 720

Archisotoma 46

Arctia 61, 68, 76, 95, 156, 158,

$213,371,548,549,560,580$

Arctinia 95, 158, 302

Arctocorisa 682

Arctornis 98, 213, 406, 560

Arcynopterix 282

Arcynopteryx 70

Arcyoptera 614

Arcyptera 366, 614, 706

Ardeola 113

Arenaria 534, 588

Arenocoris 501

Arenostola 98, 430

Arethusana 229, 315, 454, 545, $549,694,712$

Argas 445

Argiope 444, 486, 688

Argogorytes 471, 608

Argutor 631, 700

Argynnis 16, 19, 37, 61, 68, 89,

$102,104,154,158,229,236$

$315,316,358,386,387,409$

$410,430,468,492,526,545$.

$548,549,676,694,712$

Argyresthia 357, 562
Argyria 228

Argyrodes 444, 755

Argyroneta 26, 538

Argyronome 19

Argyropelecus 808

Astiphromma 336

Argyroploce 198, 229, 405, 562 Astragalus 508

Argyrotaenia 198, $405 \quad$ Astrobunus 517

Argyrotoxa $229 \quad$ Atachia 357

Argytes 511

Arholus 723

Arhopalus 746

Arhyssus 501

Ariamnes 444

Arianta 768

Aricia 19, 209, 229, 548, 549, $629,660,712$

Arion 757, 775, 776

Aristaeomorpha 619

Aristelix 193

Aristeomorpha 619

Aristeus 619

Aristotelia 386, 405

Arma 208

Armadillidium 462, 674, 675

Armadillo 462, 674

Armallidium 675

Armatosterna 290

Arnoglossus 808

Arocatus 208

Aroga 405

Aromia 185, 391

Arpaetus 608

Arragonia 187

Arrenurus 639, 687

Arrhopalites 461, 535

Arrhytmus 290

Arsilonche 61

Artabas 96

Arthrodamaeus 419, 720

Arthrodeis 100, 110, 140, 29

Arthrodinus 110, 140, 291

Arthrolips 630

Arthropoma 807

Artogeia 712

Arytaina 499

Asaphidion 38, 96, 631

Asarta 382

Asartodes 382

Ascalaphus 22, 68, 533

Ascalenia 370

Ascasis 467

Ascidia 801

Ascidiella 801

Asclera 306

Ashfordia 768

Asida 92, 113, 120, 339, 341,

$$
\text { 373, } 697
$$

Asilus 365

Asio 397, 534

Asiobates 648

Asiphum 555

Asphalia 405

Aspidapion 733, 751

Aspididris 634

Atarsos 151

Atelecyclus 710

Ateliotum 332

Atelocera 163

Atemeles 71

Atethmia 61, 98, 546, 549, 560

Ateuches 506

Ateuchus 226

Athanas 619

Athene 397, 515, 700

Atherix 506, 606

Atheroides 555

Atheta 100, 103, 436, 630

Athetis 55, 98, 189, 298, 409, 427, 560

Atheuchus 349

Atholister 723

Atholus 723

Athophora 127

Athous 88

Athripsodes 494

Athroolopha 170, 549

Atimia 746

Atinea 332

Atizies 506

Atolmis 95, 560, 580

Atomaria 63, 456, 630

Atractocerus 180

Atractus 501

Atrax 538

Atrichopogon 632

Atrina 619, 710

Atrometus 302

Atroolopha 194

Atropacarus 720, 760

Atropos 589

Atta 39, 457, 506, 634, 645

Attactagenus 751

Attacus 2, 76, 126

Attagenus 69, 179, 477, 510

Attalus 81, 140, 363

Attelabus 219, 733

Atylotus 459, 554, 586

Atypha 560

Atypus 538

Aubeonymus 681

Auchmis 98, 560

Augiades 37

Augosoma 163

Aulacaspis 633

Aulacidea 506, 653

Aulacopus 290

Aulacorthum 485, 555

Aulactinia 803

Aulax 506

Aulocera 104, 134, 358

Aspidiotus 163, 251, 364, 582, Aulogymnus 753

$$
633
$$

Aspidontus 544

Aspilapteryx 357

Aspilates 61

Aspitates 170, 228, 316, 492, 548, 549

Aspongopus 506

Astata 250, 651, 684, 685

Astenus 107

Asteriolecanium 163

Asterodiaspis 251, 650

Aulonium 484

Austromenopon 588

Austropotamobius 537 


\begin{tabular}{|c|c|c|c|}
\hline Aylax 636, 653, 668 & Bena 95, 157, 189, 228, 508, 548, & Bomolocha 98 & Brephia 382 \\
\hline Aythya 588 & 560 & Boophilus 445 & Brephos 170 \\
\hline Azanaethra 444 & Benthosema 808 & Boops 808 & Brevantennia 382 \\
\hline Azotus 251 & Beraea 494 & Borbo 315, 409, 492 & Brevicoryne 208, 485, 555 \\
\hline Azteca 541,634 & Berginus 63 & Boreaphilus 110 & Brevipalpus 346 \\
\hline Bacanius 723 & Berlesaspis 633 & Borgmeierita 634 & Brevisociaria 198 \\
\hline Baccha 232 & Bermejonia 100 & Borkhausenia 70, 370, 468, 562 & Brihaspa 506 \\
\hline Bacillus 345 & Berningerus 290 & Borocera 506 & Brinckochrysa 618 \\
\hline Bactra $198,316,405,420,562$ & Berniniella 720 & Bos 397 & Brintesia 315, 316, 386, 409, \\
\hline Badebecia 198, 562 & Berosus 77, 456, 529, 648 & Bosmina 537 & $492,545,694,712$ \\
\hline Badister 38, 96, 325, 631, 700 & Bertiella 419 & Bostrychoplites 239, 484 & Brinthesia 549 \\
\hline Baebius 602 & Bertkauia 589 & Bostrychus 88, 321, 456, 484 & Briophyla 61 \\
\hline Baeckmanniolus 456, 723 & Berytus 208 & Bothrideres 484 & Briopsis 524 \\
\hline Baetis 606, 744 & Besseria 812 & Bothriomyrmex 725 & Brithys 95,316 \\
\hline Bainzongia 485 & Betulaphis 485, 555 & Bothriophorus 600 & Broconidris 634 \\
\hline Baizongia 555 & Bezzia 632, 664 & Bothrostethus 208, 501 & Brodiella 807 \\
\hline Balearica 588 & Biareolina 320 & Bothus 808 & Brownidris 634 \\
\hline Ballistura 763 & Biastes 289, 320, 617 & Bothynoderes 391, 412, 420, 489 & Bruchidius 119 \\
\hline Balssia 27 & Bibio 81, 511 & Bothynoscelis 290 & Bruchus 44, 100, 110, 119 \\
\hline Banchopsis 704 & Bichroma 170, 430, 548 & Botocera 180 & Bruelia 534, 588 \\
\hline Banchus 336, 704 & Bidessus 161, 698 & Botochius 648 & Brumus 110, 680 \\
\hline Bangalaia 290 & Bilimneus 648 & Botrylloides 801 & Bryaxis 456 \\
\hline Banghaasia 370 & Bilobella 461, 576 & Botryllus 801 & Brychius 600 \\
\hline Bankesia 86, 382 & Biorhiza 60, 616, 753 & Boudicca 501 & Bryobia 346,445 \\
\hline Banksinoma 720 & Biosteres 514 & Bourletiella 461, 613 & Bryoleuca 524 \\
\hline Bapta 61,170 & Bipassalozetes 720 & Boyeria 647 & Bryonycta 560 \\
\hline Baptodoris 770 & Bisigilla 458 & Brachinus 631, 700 & Bryophaga 370, 406 \\
\hline Baraeus 290 & Biston 61, 170, 401 & Brachionycha 98,560 & Bryophila $61,98,154,191,228$, \\
\hline Barathra 560 & Bithynella 744 & Brachiostegia 506 & $298,427,524,546,548,549$ \\
\hline Barathraea 221, 278 & Bius 120 & Brachistomella 613 & Bryotropha 405, 562 \\
\hline Barbara 198, 405 & Bixadus 163, 290 & Brachmia 405 & Brysocrypta 215,555 \\
\hline Barbus 647 & Blabinotus 107, 110 & Brachodes 370, 468, 545, 549 & Brythys 560 \\
\hline Bardistus 506 & Blanus 113 & Brachyacma 405 & Buanea 163 \\
\hline Baris 92, 96, 391, 681 & Blaps 47, 92, 100, 110, 120, 341, & Brachycarenus 501 & Bubas 139, 186, 220 \\
\hline Barnola 589 & 412,506 & Brachycaudus $485,555,571$, & Виво 113, 397, 534 \\
\hline Barycnemis 635 & Blaptocampus 274, 302 & 670,779 & Bubopsis 22 \\
\hline Barylypa 302, 583 & Blastesthia 405 & Brachycentrus 494 & Bubulcellodes 187 \\
\hline Barytarbes 274 & Blasthopagus 183 & Brachycerus 53, 92, 681 & Bucculatrix 168 \\
\hline Basiceros 634 & Blastoblas & Brachychthonius $419,720,760$ & Bucephala 588 \\
\hline Bassus 274 & Blastodacna 370 & Brachycolus 232, 555 & Buckleria 382 \\
\hline Bathycoelia 163 & Blastophagus 141, 321, 512 & Brachycrotaphus 345 & Buffonellaria 807 \\
\hline Bathyscia 528, 724 & Blastotere 357 & Brachyderes 140, 681, 751 & Bufo 647 \\
\hline Bathysciola 27, 199, 360 & Blastothrix 251 & Brachygeophilus 620 & Buglossidium 808 \\
\hline Bathysolen 208, 501 & Blatella 311 & Brachygluta 110,456 & Bulaea 497, 649, 680 \\
\hline Batia 562 & Blatta $33,311,345,506$ & Brachyleptura 630, 746 & Bulimus 768 \\
\hline Batocera 290 & Blechrus 110 & Brachylomia 560 & Bunaea 506 \\
\hline Batodes 198, 405 & Bledius $63,100,110,412,470$ & Brachymeria 376, 799 & Bunodactis 803 \\
\hline Batomena 290 & Blennius 808 & Brachymyrmex 634 & Bunodes 803 \\
\hline Batozonus 127 & Blepharidatta 6 & Brachynema 125 & Bunodosoma 803 \\
\hline Batrachedra 562 & Blepharidopsis 365 & Brachynus $15,38,96,100,110$ & Bupalus $170,194,228,302,430$, \\
\hline Batrachorhina 290 & Blepharipus 608 & Brachyolene 290 & 549 \\
\hline Batrisodes 456 & Blepharita 427, 492, 545, 560 & Brachypelta 125, 208 & Buprestis 88, 119, 140, 219, 434 \\
\hline Baudonia 775,776 & Blepisanis 290 & Brachypogon 632, 664 & Burriola 242 \\
\hline Bazaria 382 & Blitopertha 214 & Brachyptera 282, 707 & Bursoplophora 720, 760 \\
\hline Bebelothrips 638 & Blitophaga 338, 511 & Brachypterolus 512 & Buskea 807 \\
\hline Beckerella 461 & Boarmia 61, 170, 189, 228, 545, & Brachypterus 512 & Buteo $397,515,534,588$ \\
\hline Bedelia 332 & $546,548,54$ & Bradyrrhoa 382 & Byrsocrypta 485 \\
\hline Belba 720 & Boernella 461 & chendyla 620 & Bythinus 110 \\
\hline Belisarius 82 & Boerner & gus 608 & Cabalia 100, 138 \\
\hline Bellicositermes & Boethus & la 20 & Cabera 170, 228 \\
\hline Belomicrus 365, 608 & Boetica 242 & mella 461 & Caccobius 237, 477 \\
\hline Belonogaster 33 & Boisduve & Brachythemis 574 & Cacoecia 270 \\
\hline Belonopelta 63 & Bolbelast & Brachytrypes 506 & Cacoecimorpha 198, 405, 468, \\
\hline Belopus 110,4 & Bolitochara 1 & Brachyunguis 485,555 & $545,546,549$ \\
\hline Belorchester 7 & Bolitophagus 37 & Brachyuro 619 & Cacophya 370 \\
\hline Belorchestes 419, 720 & Boloria 104, 195, 315, 430, 526 & Bracon 193, 375, 446 & Cacospongia 800 \\
\hline Belostoma 506 & Bolorra 189 & Bradybaena 768 & Caddo 517 \\
\hline Bembecia 86 & Bombix 525, 538 & Bradycellus 38, 96 & Cadra 382 \\
\hline Bembecinus 608 & Bombus 33, 127, 175, 289, 512, & Bradyrrhoa 545 & Caecilius 589 \\
\hline Bembex 127, 129, 222 & 780 & Bradytus 96, 631 & Caedius 240 \\
\hline Bembidion 38, 92, 96, & Bombux 543 & Braquinus 71 & Caenacis 616, 636 \\
\hline 700 & Bombyces 429 & & Caenia 262 \\
\hline Bembidium 470 & Bombyx 85, 101, 133, 158, 192, & Brenthis 29, 61, 74, 89, 104, 11 & Caenis 506, 744 \\
\hline Bembis 250 & $213,236,283,298,333,371$, & $195,315,358,386,409,43$ & Cafius 110 \\
\hline Bembix 608, 621, & $406,426,444,506,546,548$, & $454,492,526,548,676,694,712$ & Cajalecanium 650 \\
\hline Bemisia 232 & 603 & Brentis 37, 68 & Calamia $98,136,560$ \\
\hline
\end{tabular}


Calamoceras 494

Calamotropha 382

Calandra 100, 110, 312

Calandria 71

Calappa 710

Calathidius 110

Calathus 38, 62, 92, 96, 107, 110, $140,631,700$

Calephorus 345

Calepitrimerus 346

Caleremaeus 708, 720

Calidris 588

Caliptus 512

Callaphis 485

Callestege 545

Callichroma 290

Calliclystis 170

Callicnemis 139, 186, 214, 600

Callicrania 614, 706

Callidiellum 746

Callidium 183, 746

Callierges 95

Calliergis 560

Callimation 290

Callimorpha 95, 158, 302, 371 $548,549,560$

Calliocoris 602

Callionymus 808

Calliphora 179, 463, 477, 500, 505

Calliptamus 138, 226, 345, 614, 672, 706, 716

Callirhytis 668, 769

Callispa 163

Callistege 560

Callisto 357

Callistodema 602

Callophris 89

Callophrys 19, 61, 93, 315, 316, 409, 430, 548, 549, 660, 712

Callopistria 560

Calloptenus 614

Callosobruchus 443

Callymorpha 68

Calocalpe 154

Calocasia 213, 524

Calocoris 125

Calophasia 61, 95, 98, 113, 548, 560

Calopsectra 587

Caloptenus 614

Calopterus 506

Calopteryx 396, 543, 647

Caloptilia 357

Calosoma 71, 75, 88, 100, 171 321,700

Calospilos 170, 549

Calostigia 130, 170

Calothysanis 61, 170, 548, 549

Calpe 98

Calvia 649, 680

Calymnia 98, 549

Calypticus 650

Calyptomerus 600

Calyptonotus 125, 208

Calyptorhyna 266

Calyptra 560

Camenta 163

Camilla 756

Caminara 110

Camisia 419, 708, 720, 760

Campalita 220

Campichoeta 756

Campodea 542

Camponotus 18, 39, 455, 457

$466,506,543,600,634,641$,

$645,656,692,715,719,725$,

$765,767,802$
Campoplex 129, 274, 711

Campta 570

Camptocladius 532, 785

Camptogramma 170, 228, 430, 548,549

Camptonupne 290

Camptopoeum 289

Camptopus 125, 208, 501

Campylister 723

Canariella 240

Canariola 345

Candidula 768, 782

Canis 113, 397

Cantharis 306, 456, 681

Cantharocnemis 290

Canthocamptus 537

Capella 397

Capitophorus 485, 555

Capnia 282, 707

Capnopsis 707

Cappeia 382

Capra 113, 314, 726

Caprimulgus 534

Capros 808

Capsus 232

Carabodes 419, 708, 720

Carabus 15, 38, 62, 68, 71, 75

$88,92,107,261,325,342$, $359,407,700,804$

Caradrina 55, 61, 98, 157, 189, 190, 191, 228, 229, 298, 300, $395,427,492,545,546,549$, 560

Carambis 602

Carapus 808

Carassius 264

Carcharodus 37, 61, 101, 315,

316, 386, 409, 410, 492, 546,

$548,549,660,712$

Carcina 370,545

Carcinops 219, 456, 723

Cardepia 524, 545, 560

Cardiocondyla 634, 802

Cardiola 630

Cardiomera 325

Cardiophorus 96, 100, 391, 630

Cardiostichus 119

Carduiceps 588

Carebara 506, 634

Carebarella 634

Carinomyrmosa 722

Carinostigmus 652

Carminodoris 770

Carphophilus 512

Carpocapsa 44, 302

Carpocoris 125, 208

Carpoglyphus 445

Carpophilus 119, 120, 551

Carposina 198, 405

Cartallum 391

Carterocephalus 315

Carterus 38, 631, 700

Cartodere 87, 630

Carulaspis 633

Caryocolum 405

Caryophyllia 770

Casilda 170, 549

Casinaria 336, 689

Cassida 13, 15, 140, 219, 304,

$$
420,446,456
$$

Catabola 332

Cataclysme 61, 170, 229

Cataclysta 382, 481

Catagapetus 494

Cataglyphis 457, 600, 715, 719 $725,767,802$
Catalanura 749

Catamachilis 575

Cataphronetis 47, 100, 110, 120, 291, 341

Catapion 733, 751

Cataremna 382

Cataretes 512, 551

Catarhoe 170

Catascia 234

Catasphalma 382

Catataxia 290

Catephia 61, 98, 560

Catharosia 812

Cathormiocerus 681, 697

Catocala 61, 76, 98, 113, 229

$236,316,333,358,406,430$

453, 468, 492, 508, 509, 524,

$545,546,548,549,560$

Catoglyptus 336

Catomulus 100

Catomus 47, 100, 120, 341

Catopidius 360, 528

Catops 360, 456

Catoptria 149, 228, 382, 548

Caupolicanas 499

Cavariella 485, 555, 571

Cavia 397,502

Cavoptinus 748

Cebrio 600, 697

Cecidostiba 616

Cecilioides 757, 782

Cecropis 113

Cedestis 370

Celaeno 720

Celama 95, 229, 316, 546, 549, 560

Celastrina 19, 104, 228, 315

316, 410, 430, 455, 468, 492, $548,549,660$

Celerio 9, 20, 41, 56, 61, 73, 76,

$101,102,316,409,430,468$, 548, 549, 559

Celipha 405

Cellepora 807

Celleporina 807

Celonites 296

Celyphoides 198, 405, 562

Cemiostoma 163

Cemonus 572

Cenopalpus 346

Centorrhynchus 621

Centris 637

Centrocoris 208, 501

Centrolene 787

Centromyrmex 634

Centrophlebomyia 179

Centroribates 419

Centroscelicoris 602

Centroscelis 602

Cepaea 768, 782

Cephalochalcidia 799

Cephalochilus 584

Cephalonomia 163

Cephalotes 634

Cephalothrips 638

Cephenium 63

Cepheus 720

Cephimallota 332

Cephis 170

Cepola 808

Cerachipteria 720

Ceracia 204

Ceraleptus 208, 501

Cerambyx 88, 290, 321, 414, 506, $569,630,746$

Ceramius 129

Cerapachys 634

Cerapterocerus 364

Cerapteryx 95, 560
Cerascopus 602

Cerasphorus 290

Cerastis 95, 427, 548, 560

Cerataphis 485

Ceratapion 733, 751, 789

Ceratia 204

Ceratina 33, 129, 289, 552, 617, 758,780

Ceratitis 116, 163, 227, 233, 372, 505, 540, 578, 579, 595, 596, $604,605,610,611,622,624$, 626, 642

Ceratobezzia 777

Ceratogastra 704

Ceratophyllus 50

Ceratophysella 461, 735

Ceratophyus 237

Ceratopogon 632, 664

Ceratoppia 720

Ceratoscopelus 808

Ceratothrips 615

Ceratozetes 419, 720, 741

Cerceris 81, 127, 250, 489, 531, $608,621,685,713,722$

Cercidia 688

Cercus 551

Cercyon 456, 699, 762

Ceriagrion 396, 647

Ceriodaphnia 537

Cerioides 216, 309, 450

Cernuella 757, 768, 782

Cerocala 98, 298, 560

Cerocoma 58, 138, 201

Ceroctis 100, 138

Cerodontha 337

Ceromastix 602

Cerophytum 600

Ceroplastes 163, 208, 251, 650, 722

Ceroplesis 100, 163, 290

Cerostoma 357

Cerula 643

Cerura 61, 90, 316, 406, 466,

$468,545,549,563,643$

Cerylon 484

Cetonia 139, 186, 214, 220, 321, 630, 681

Ceuthorhynchus 140

Ceuthorrhynchus 100

Ceuthosphodrus 62, 82, 103

Ceutorhynchus 751

Chabuata 95

Chaenius 38

Chaenon 193, 337

Chaenusa 193

Chaetabraeus 723

Chaetarthria 648, 699

Chaetechelyne 620

Chaetocnema 219, 260, 304, 420

Chaetococcus 666 
Chamaepsila 784

Chamaesphecia 74, 86

Chamaesyrphus 394

Chamobates 720

Chaoboris 506

Chaoborus 11

Charadrius 397, 534, 588

Charadrus 588

Charanyca 560

Charaxes $45,91,104,236,315$,

$316,385,409,482,526,545,548$

Charcharodus 19, 229

Chariclea 61, 95, 157

Chariesthes 163, 290

Charimachilis 575

Charops 336

Chasmatopterus 214, 697

Chasmodes 372

Chazara 229, 315, 409, 430, 468, $549,629,694,712$

Cheiloporina 807

Cheilosia 231, 309, 394

Cheimophila 370

Chelidura 344

Cheliomyrmex 634

Chelis 89, 95, 545, 560, 580

Chelon 744

Chelonitis 680

Chelonus 375, 711

Chelostoma 289, 637

Chelystruma 634

Chemerina 61, 170

Chermes 251, 650, 666

Chersotis 95, 189, 228, 468, 492, $524,545,546,560$

Chesias 61, 170, 545, 548

Cheumatopsyche 494

Chevrolatia 391

Chiasmia 170, 228

Chilapion 733, 772

Chilo 45, 143, 299, 382, 406, 430,

Chilocorus 15, 232, 518, 582, 680

Chilodes 560

Chiloneurus 25

Chilosphex 684

Chilostoma 768

Chilotoma 258, 266

Chilotomina 260, 266, 276, 411

Chimarra 494

Chinaspis 633

Chionaspis 251, 633

Chionodes 405

Chiracanthium 538, 709

Chirocampa 229

Chironitis 28, 237

Chironomus 24, 32, 42, 46, 532, $565,587,606,752$

Chirothrips 609

Chitona 818

Chlaeniellus 391, 631

Chlaenius 38, 96, 100, 631, 700

Chloantha 98, 56

Chlorapion 733

Chloridea 61, 95, 102, 508, 524, $545,548,549$

Chlorissa 61, 170, 228, 298, 409, 549

Chloroperla 282, 707

Chlorophorus 630, 681

Chloroprocta 732

Chloropulvinaria 650

Chlorostola 258, 266, 276

Chlorotocus 619

Chnaurococcus 666

Choleva 360, 528
Chondrostega 61, 101, 189, 190 406, 430, 492

Chondrostoma 744

Chondrula 757

Chorebidea 193, 337

Chorebus 193

Chorinaeus 263

Chorioptes 445

Chorischizus 129

Chorizopora 807

Chorosoma 501

Chorthippus 345, 612, 614, 706, 716, 727

Chortophila 330, 365, 514

Chrisomela 78

Chroantha 208

Chromacilla 290

Chromalizus 290

Chromaphis 485

Chromoderus 391

Chrysapion 733, 772

Chrysaspidia 430

Chrysiridia 76

Chrysis 129, 175, 440, 458, 513 , $572,713,820$

Chrysobothris 88, 225

Chrysocambrus 382, 430

Chrysocarabus 62, 119, 261

Chrysochloa 697

Chrysochraon 614

Chrisoclista 370

Chrysocrambus 316, 548

Chrysodeixis 427, 560, 71

Chrysogaster 231, 394

Chrysolina 219, 307, 456, 681, 697

Chrysomela 9, 92, 107, 110,113, 260, 304, 307

Chrysomella 113

Chrysomphalus 364, 633

Chrysomya 477, 714, 732

Chrysomyia 506

Chrysopa 22, 215, 232, 335, 364

$375,396,566,590,591,618$. 686, 747

Chrysoperla 618, 686

Chrysophanus 19, 56, 61, 89,

$$
113,196
$$

Chrysops 163, 554, 586

Chrysoptera 98

Chrysoteuchia 382, 549

Chrysotoxum 309, 394, 500

Chrysozona 554, 586

Chydorus 537

Chyromya 756

Cicada 113, 271, 284, 506

Cicadula 216

Cicindela 15, 62, 92, 96, 100, 113 ,

$118,220,261,506,600,700$ Cicindella 395, 412

Ciclyrius 712

Ciconia 397

Cidaria 61, 134, 170, 189

Cilicornella 332

Ciliella 768

Cilindrojulus 420

Cilindronotus 92, 107

Cilix 61, 90, 405

Cimex 37, 59, 66, 501

Cimipsa 100

Cinara 485, 571

Cinaropsis 485

Cincinata 290

Cinclus 397

Cinglis 170, 549

Cintameva 22

Ciona 801
Circaetus 397, 588

Circapion 733, 772

Circus 397, 515, 534

Cirrhia 228, 492, 524, 545, 546, 560

Cis 630

Cissister 723

Cistapion 733

Cisticoccus 650

Cisticola 113

Cistogaster 812

Citharus 808

Cladocerotis 95, 548, 549, 560

Cladognathus 506

Cladotanytarsus 532, 565, 587 $665,703,717$

Clambus 600

Clania 506

Clanoneurum 420

Clasperia 382

Clavelina 801

Clavigesta 149, 198, 405

Clematostigma 589

Cleoceris 524, 560

Cleonymia 560

Cleonus 96, 391, 489, 53

Cleophana 61, 95, 155, 191, 316 , $492,545,548,549,560$

Clepsis 198, 388, 405, 545, 549

Cleptes 347

Cleta 61, 170

Clinia 290

Clinohelea 777

Clisodon 289

Clitobius 100, 291

Clitostethus 680

Clivina 38, 62, 96, 631

Cloeon 264

Cloniophorus 290

Clossiana 104, 195, 315, 409

$430,492,526,549,676,694$

Clostera 492, 563, 643

Clubiona 709

Clunio 46, 665

Clysia 9

Clythopsis 204

Clythra 258, 260, 411

Clytie 61, 98, 228, 549, 560

Clytra 260, 266, 278

Clytus 15, 183

Cnemapion 733

Cnemeplatia 240, 34

Cnemodon 394

Cnemolia 290

Cnephasia 168, 198, 405, 545, 546, 562

Cnephasiella 198

Cnethocampa 41

Coccidiphaga 98, 136, 191, 371, 560

Coccidiphila 370

Coccidula 680

Coccinela 78

Coccinella 92, 110, 140, 232, $321,456,497,512,571,582$ $603,649,680,681$

Coccinula 582, 649, 680

Coccophagoides 251

Coccophagus 518

Coccotrypes 551

Coccus 251, 364, 633, 650, 666

Cochlicella 757, 768, 782

Cochlicopa 782

Cochlidion 386

Cochliomya 578, 611, 626

Cochliomyia 714, 732

Cochlioteca 382

Cochlyomia 622

Cochranella 787

Cochylidia 198, 405

Cocytius 480

Codiomyrmex 634

Codioxenus 634

Codophila 125, 208

Coelaenomenodera 163

Coelambus 100, 122, 161, 184

Coelinius 193, 337

Coelioxys 289, 637

Coelocephalapion 733

Coeloconus 336

Coelogaster 249

Coelopisthia 689

Coelopterus 680

Coelorhynchus 808

Coelostoma 100, 456, 648

Coelotanypus 752

Coenagrion 264, 396, 647

Coenobia 98, 560

Coenocalpe 170

Coenonympha $16,37,61,89$

104, 113, 134, 181, 190, 229,

$315,386,409,410,430,468$,

492, 545, 548, 549, 629, 660, 694, 712

Coenostegia 506

Coenotephria 468

Coenothele 486, 538

Colaspidema 277, 456, 681

Coleophora 168, 357, 387, 492 562

Colias 12, 20, 37, 56, 61, 89, 93, $104,113,157,194,229,236$ $314,315,316,358,386,406$ $409,410,430,492,509,526$, $545,548,549,550,629,659$, $660,694,712$

Colinus 588

Collartida 602

Colletes 138, 218, 288, 289, 458, 499, 654, 758, 780

Colletosia 807

Collicularia 405

Colobatus 501

Colobochyla 98, 524, 560

Colobopsis 506

Coloburella 461

Colocasia 98, 560

Colopha 485

Coloradia 506

Coloradoa 485

Colotes 363, 456

Colotis 61, 157, 428, 430, 468 $526,659,693$

Colotois 170, 228, 549

Colpocephalum 397, 534, 588

Colpoderus 290

Colpognathus 336 
Compsomyia 179

Compsoptera 61, 170, 298, 548, 549

Compsothrips 638

Comsomyia 179

Comstockiaspis 251

Conapion 733

Conapium 73

Conger 808

Coniatus 100

Coniocleonus 92, 110, 391, 681

Coniocompsa 806

Coniopteryx 22, 567, 591, 618, 686,806

Conisania 560

Conistra 61, 98, 136, 158, 211, $228,427,524,560$

Conizonia 569

Conocephalus 345, 614, 706

Conomyrma 634

Conops 500

Conorhynchus 110, 489

Conorrhynchus 420

Conosomus 103

Consivius 501

Contigaspis 633

Conwentzia 22, 591, 618, 686, 806

Copelatus 391

Copiphana 95, 560

Copium 125

Copris 139, 237, 681

Coptocephala 258, 260, 266, 276

Coptochilus 723

Coptops 163, 290

Coptosoma 208

Coptostethus 110

Coptosthetus 140

Coracias 397

Coranus 125, 176, 208, 602

Corbicula 786

Corcyra 382

Cordilomera 180

Cordulegaster 647

Cordulia 264

Cordylomera 290

Corempis 602

Corethra 11, 46

Corethrogaster 290

Coreus 208, 501

Coridius 506

Coriomeris 208, 501

Coris 808

Coriscus 501

Corisella 506

Corixa 131, 682

Corizus 208, 501, 556

Cornicacoecia 198

Cornifrons 382

Cornuaspis 633

Coroebus 630

Coronella 794

Corticaria 63, 630

Corus 290

Corvus 397, 489, 534

Corymbia 630, 746

Coryna 100, 138, 501

Corynoneura 32, 532, 565, 785

Coscinia 61, 95, 154, 157, 158

$229,409,492,523,545,548$,

$549,560,580$

Cosmia 61, 98, 113, 316, 427, $546,549,560$

Cosmina 262

Cosmobunus 517

Cosmochthonius 419, 445, 720, 760
Cosmolestes 602

Cosmopolites 312

Cosmopteryx 370

Cosmotriche 314

Cossiphus 15

Cossus 41, 45, 61, 76, 86, 371, 405

$506,545,548,549,551,570$

Cossyphodes 110

Cossyphus 15, 47, 174

Costaconvexa 130,170

Costiella 602

Cosymbia 170, 211, 298, 316, 548, 549

Cotiheresiarches 316

Crabo 129

Crabro 250, 365, 608, 651, 722

Crambus 194, 209, 228, 229 382,430

Crangon 619

Craniophora 98, 524, 560

Craspedorrhynchos 588

Craspedorrhynchus 534

Craspedum 501

Creightonidris 634

Creightoniella 637

Cremastus 129

Crematogaster 18, 39, 457, 506

$634,645,715,719,725,802$

Creoleon 22, 396, 590, 591, 618, 686

Creophilus 179

Crepidodera 260, 304, 456

Cribrilaria 807

Cribrilina 807

Cricotopus 32, 46, 532, 565, 703 , 785

Crino 98

Crinopteryx 332

Criocephalus 88, 391, 414

Crioceris 260, 456

Criococcus 666

Criorrhina 231

Criphia 546

Crisia 807

Crocallis 61, 170, 228, 467

Crocidosema 198, 298, 405

Crocisa 320

Crocistethus 208

Crocota 170, 229

Crocothemis 205, 264, 396, 647

Crocrothemis 205

Crocuta 365

Croesia 198, 405

Crombrugghia 382

Crosisa 289

Crossocerus 608, 651

Crossotofrea 290

Crossotus 290

Crowsoniella 600

Crunoecia 494

Crymodes 98, 560

Cryphia 492, 524, 545, 560

Cryptacarus 419

Cryptarcha 512

Cryptaspidiotus 633

Crypticus 47, 88, 92, 107, 110, $120,140,159,341,412$

Cryptoblabes 235, 382

Cryptocephalus 219, 260, 277,

$304,456,469,681$

Cryptocerus 767

Cryptochilus 81

Cryptochironomus 587

Cryptogalumna 419

Cryptolaemus 78, 119, 235, 680

Cryptolechia 370
Cryptomorpha 119

Cryptomyzus 555, 745

Cryptophagus 82, 630

Cryptopleurum 699, 762

Cryptopone 634

Cryptops 620

Cryptopygus 535

Cryptorrhynchus 372

Cryptosaccus 768

Cryptoscenea 806

Cryptotendipes 785

Cryptothrips 638

Cryptus 263, 319, 336, 372

Crysis 127

Crysomphalus 582

Ctenicella 801

Ctenichneumon 667

Ctenobelba 720

Ctenocephalides 50

Ctenochares 319

Ctenocnemis 602

Ctenodecticus 345

Ctenopelma 274, 336

Ctenoplusia 560

Ctenopus 81, 138

Ctenosta 110

Ctenus 538

Cuclotogaster 588

Cucujus 308

Cuculia 302, 509

$\begin{array}{ll}\text { Cuculia 302, } & \\ \text { Cucullia 41, 61, 95, 190, 228, } & \begin{array}{l}\text { Cytilosoma } 723 \\ \text { Cytodictes } 445\end{array}\end{array}$

316, 372, 492, 524, 545, 546, Daceton 634

$548,549,560$

Cucullococcus 666

Culex 11, 97, 162, 378, 627, 754

Culicimimus 602

Culicoides 14, 632, 664

Cultroribula 720

Cummingsiella 588

Cunaxa 445

Cunctochrysa 618,686

Cuneopalpus 589

Cupes 600

Cupido 104, 228, 315, 409, 455, $508,545,549,660,712$

Cupressaspis 633

Curculio 399, 681, 733

Curtoneura 179

Curtonotum 756

Curtonotus 631

Cyanapion 733, 751

Cynaeda 545

Cyaniris 19, 209, 258, 276, 549, 712

Cybister 122, 161, 184, 264, 506

Cybocephalus 140, 512

Cybopiestes 114

Cybosia 95, 560, 580

Cychramus 512, 570

Cychrus 62, 75, 88, 199

Cyclocosmia 538

Cycloderes 110, 391, 681, 697

Cyclonotum 77, 648

Cyclopelta 506

Cyclophora 467, 545

Cyclops 11, 537

Cyclosa 688

Cycnia 95, 430, 560, 580

Cydia 562

Cylindromyia 812

Cylindromyrmex 634

Cylindronotus 47, 88, 110, 120

Cylindropsis 7, 27

Cylindroptinus 748

Cylindrothorax 100, 138

Cylister 373, 723

Cylistosoma 723

Cyllenia 347

Cyllodes 512, 570

Cymatia 682

Cymatophora 90

Cymbalophora 158

Cymbalorissa 382 601, 631

Cymotricha 405

Cymus 125, 208

Cynaeda 382

Cynegetis 680 668, 769

Cynomyia 179

Cyphalonolus 444

Cyphomyrmex 634

Cyphon 456

Cypridopsis 537

Cypsela 179

Cyrnus 494

Cyrtoptyx 616

Cystodytes 801

Cyta 445

Dacne 88, 19

Dacnusa 193, 337

Dactylopius 666

Dactynotus 555

Damaeolus 720

Damaeus 720, 795

Dameobelba 720

Dameosama 488

Dameosoma 720

Danacea 219

Danais 56 628, 646

Daphnia 537

Dapsa 119, 217

Darwinella 800

Dascillus 600

Daseochaeta 98

Dasia 485

Dasycampa 524

Dasycella 370

Dasycepa 562

Dasycera 386, 545

Dasycerus 600

Cymbaeremaeus 708, 720

Cymindis 38, 62, 140, 171, 359,

Cynips 60, 439, 616, 636, 653,

Cynthia 492, 628, 676

Cyphoderus 461, 613

Cyrtonus 238, 681, 697

Dactylocladius 532, 565

Dactylosternum 110, 456

Dacus 116, 421, 423, 447, 465, 475, $505,558,579,610,611,622$

Danaus 236, 314, 385, 543, 544,

Daphnis 76, 101, 468

Daptus 38, 322, 391, 412

Daraba 298, 299, 382

Dasychira 98, 136, 213, 372, 560 


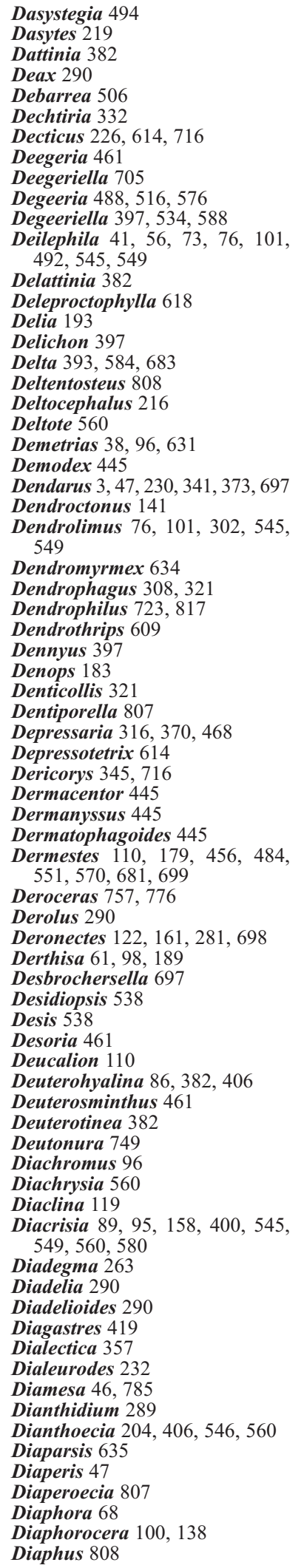

Diapterobates 708

Diarsia 95, 524, 560

Diarthrothrips 163

Diasemia 382

Diasemiopsis 382, 549

Diaspidiotus 251, 633

Diaspis 251, 498, 582, 633

Diastata 756

Diastictis 170

Diastictus 92, 139, 600

Diastixus 120

Diastrophus 653

Diazona 801

Dicarnosis 364

Dicatozetes 419

Diceratura 198, 229, 405

Dicerca 88,108

Dicestra 545, 546, 548, 560

Dichillus 47, 120, 341, 373

Dichirotrichus 38, 96, 110

Dichocarabus 75

Dichomeris 228, 405

Dichonia 98, 228, 560

Dichorista 546

Dichostathes 290

Dichrodontus 107

Dichromerus 501

Dichrorampha 405, 562

Dicologlossa 808

Dicranocephalus 208, 501

Dicranolasma 517

Dicranomerus 501

Dicranopalpus 517

Dicranota 606

Dicranura 41, 61, 73, 127, 402, 448, 466, 493, 509

Dicrodontus 110

Dicrorampha 198

Dicrotendipes 587, 752

Dictyna 538

Dictyogenus 707

Dictyopterus 600

Dicycla 98

Dicyla 545, 560

Dicyrtoma 461

Dicyrtomina 46

Didemnum 801

Didinus 180

Dienoplus 608, 621

Dilar 22

Diloba 302, 400, 524, 545, 549, 560

Dilta 575

Dimerocerus 723

Dinetus 651

Dinocampus 571

Dinocras 282, 707

Dinoderus 484

Dinodes 325

Dinoponera 634

Diodontus $129,250,499,652$ 684,713

Diogenes 710

Diorchestes 720

Dioryctria 224, 314, 382, 406, 492

Diospilus 512

Dioxys 127, 289, 637

Diphaglossa 499

Diphtera 560

Diphthera 213, 524

Diphyllaphis 460, 485

Diphyllus 199

Diplacodes 264, 574

Diplapion 733

Diplazon 319, 667

Diplectrona 494
Diplocerus 502

Diplocoelus 199

Diplocopholus 444

Diplocyrtus 114

Diplodontus 687

Diplodus 808

Diplolepis 60, 636, 668

Diplorhoptrum 634, 715, 719, 725,802

Diplosolen 807

Diplosoma 801

Diplura 61, 101, 190

Diplurella 545

Diprion 558

Dipsosphecia 61, 86, 549

Dipterygia 98

Dira 229

Diretmus 808

Discestra 228, 300, 316, 468, 492

Discoceps 290

Discodiaspis 633

Discodoris 770

Discoelius 296, 393

Discolops 290

Discothyrea 634

Dismorphia 450

Disonicha 119

Disorhina 720

Disporella 807

Dissoctena 86, 382

Disstroma 211

Distagmos 357

Distaplia 801

Distoleon 686

Distomus 801

Ditela 800

Ditomus 38, 96, 631

Ditrymacus 346

Diuraphis 485, 555

Diurnea 370

Divales 681

Divona 382

Dixippus 318

Dixus 631

Dizygomiza 337

Dizygomyza 193

Dociostaurus 138, 183, 345, 478 , 614, 706, 716

Docophorus 534, 588

Dolamara 240

Dolerocypris 537

Dolicharthria 382, 548, 549

Dolichoderus 634, 656, 725, 765

Dolichopus 365

Dolichovespula 296

Dolichus 261

Doliopygus 180

Dolomedes 486, 503, 538

Dolycoris 208

Dometorina 419,708

Donacia 257, 41

Donus 681

Doralina 555

Doralis 485, 571

Dorcadion 110, 361, 391, 681, Ebaeus 363, 437, 470 697, 764

Dorcatoma 324

Dorcus 88, 186, 214, 630

Doriphora 78

Dorisidris 634

Dorthezia 666

Dorycranosus 720

Dorylus 725

Dorymyrmex 634

Doryphora 9

Dorypteryx 589

Drasterius 110, 391

Dryocosmus 558
Dorysthenus 506

Dorytomus 391

Doto 730

Douglariella 666

Douglasia 357, 666

Douglasiella 666

Drapetes 600

Drepana 61, 74, 89, 90, 315, 405, $429,545,549$

Drepanosiphum 232, 485

Drepanothrips 609

Dreyfusia 571

Drilus 456, 600

Dromius 38, 96

Dromoela 113

Drosophila 163, 226, 318, 505, $543,610,611,626$

Drusus 494

Dryas 76

Drylus 71

Drymonia 90, 563, 643

Dryobota 98, 524, 560

Dryobotodes 61, 98, 524, 545, 560

Dryocoetes 512

Dryophilus 140

Dryops 77, 107, 456, 529, 600

Drypta 96, 631

Dryseriocrania 80

Dryudella 621, 651, 684

Dubreuilli 729

Dufourea 289, 637, 654

Dugesia 813

Dundubia 506

Duplachionaspis 633

Duponchelia 382, 545

Duvalius 360, 568

Dynaspidiotus 633

Dynastes 139

Dypterygia 560

Dyrphia 290

Dysaphis 485, 555, 745, 779

Dysauxes 90, 429, 560

Dyscestra 386

Dyschirius 38, 96, 100, 110, 631, 700

Dyscia 61, 158, 170, 228, 468, 545

Dyselachista 357

Dyseriocrania 357

Dysgonia 548, 560

Dysidea 800

Dysmasia 332

Dysnicoccus 666

Dyspessa 61, 86, 191, 405, 430, 492, 548, 549

Dysspastus 382

Dystebenna 370

Dytiscus 15, 122, 184, 264, 506, $529,648,698$

Eana 198, 405

Earias 95, 228, 298, 299, 468, 508, 545, 548, 549, 560

Earophila 170

Ebulea 382

Ecacanthothrips 216

Ecclisopteryx 494

Eccopisa 382

Eccoptomera 756

Ecdyonurus 744

Echinodera 140

Echinogammarus 744

Echinosomidia 110

Echthromorpha 656

Eciton 457, 634 
Ecliptopera 467

Ecnomorphus 551

Ecnomus 494

Ecphroroma 100

Ecpyrrhorrhoe 382

Ectatomma 634

Ecteinascidia 801

Ectemnius 608, 621, 651, 685

Ectilis 193

Ectobius 345

Ectomocoris 602

Ectomyelois 235, 316

Ectophasia 812

Ectopsocus 589

Ectriochodia 602

Ectromopsis 697

Ectypa 228, 229

Edaphopaussus 600

Egadroma 38, 96, 631

Egea 549

Egira 560

Eidocolastus 55

Eidophasia 357

Eilema 95, 191, 228, 229, 298 , $316,545,548,549,560,580$

Eirenis 794

Eitella 382

Elachista 229, 357

Elampus 572

Elaphe 794

Elaphocera 28, 139, 214

Elaphria 55, 560

Elaphrus 62, 160

Elasmostethus 208

Elasmus 375

Elastrus 110

Electrophaes 170

Eledona 47, 373

Eledonoprius 373

Elegia 382

Elenophorus 47, 341, 412

Elfia 812

Eligmodonta 643

Elipsocus 589

Elithiodes 290

Elkneria 213

Ellampus 129, 440, 572

Ellipsodes 110, 159

Elliptosoma 110

Ellopia 170, 549

Elmis 506, 744

Elona 768

Elymas 602

Ematheudes 382

Ematurga 170, 229, 468, 549

Emblethis 125

Emesodema 602

Emmelia 229, 560

Emphanes 96, 63

Empicorella 602

Empicoris 602

Emprepes 382, 549

Emys 113

Ena 782

Enargia 98, 386, 430, 546, 560

Enargopelte 518

Enarmonia 198, 405

Encomista 30, 549

Enconista 30, 61, 170, 409, 492 , 545,548

Encopognathus 651

Encyrtos 375

Encyrtus 251, 364

Endochironomus 785

Endomia 219, 456
Endomychus 217

Endophloeus 88

Endothenia 198, 405

Endothricha 228

Endotricha 229, 382, 430, 545, 548,549

Endromis 377, 382, 433

Endrosis 70, 370

Endurus 336

Enicmus 63, 456, 630

Eniochthonius 419, 720

Ennomos 61, 170, 467, 545, 549

Enolmis 370

Enoplium 484

Enoplops 50

Entalophoroecia 807

Entephria 170, 467, 468

Entomobrya 461, 474, 488, 516,

$$
535,576,613
$$

Entomognathus 608, 65

Eobania 768

Eobrachychthonius 419, 720

Eoglaresis 267

Eopelobates 787

Eoperla 707

Eophila 781

Eotachys 96, 631, 700

Eotetranychus 346, 445

Epacromia 614

Epagoge 405, 562

Epagone 198

Epeira 26, 444

Epeolus 289, 617

Epermenia 357

Ephebomyrmex 634

Ephedraspis 633

Ephedrus 670

Ephemerella 744

Ephesia 61, 98, 157, 197, 549

Ephestia 6, 164, 287, 301, 318, $382,548,611,711$

Ephialtes 274, 336, 372

Ephialtites 765

Ephippiger 366, 614

Ephippigera 614

Ephippigerida 345, 614, 706

Ephutomma 722

Ephydria 506

Ephysteris 405

Epiblema 149, 198, 405, 562

Epicallia 158, 191, 431, 468, 545, 560,580

Epicauta 138

Epichnopterix 86, 382

Epicnaptera 61, 101, 155

Epicorsia 382,

Epidamaeus 795

Epidauria 382

Epidiaspis 633

Epidola 405

Epierus 723

Epilachna 44, 92, 603, 680

Epilecta 95, 560

Epilohmannia 419, 720, 760

Epimecia 98, 560

Epimipta 193

Epimyrma 457, 725

Epinephele 16, 61, 113, 229, 236

Epinotia 149, 198, 405, 562

Epipsocus 589

Epipyrops 86, 405

Epirrhoe 170, 545

Epischnia 382, 409

Episema 98, 213, 406, 492, 524

$545,546,548,549,560$

Episernus 324
Epistrophe 231

Epitheca 264

Epithectis 40

Epithrix 304

Epixenus 767

Epizeuxis 98, 560

Epoicocladius 532, 565

Epomis 96

Eporibatula 419, 708, 720

Epuraea 512, 570

Epurea 217

Equus 397

Erannis 45, 61, 170

Erastria 61, 98, 191, 409, 427, 549

Erebia 29, 37, 68, 74, 80, 89, 104 ,

$113,189,194,195,196,229$

$315,316,409,430,492,508$,

$525,547,548,549,597,694,712$

Eremaeus 708,720

Eremobelba 720

Eremobia 98, 228, 468, 524, 549 560

Eremochlaena 427, 524, 560

Eremocoris 125

Eremodrina 524

Eremopola 61, 98, 524, 546, 560

Eremovescia 602

Eresus 444, 463

Eretes 100, 161, 506

Eretmocera 370

Eretmotus 723

Ergates 88, 321, 746

Erigorgus 274, 302

Erimyrmosa 722

Eriococcus 251, 666

Eriocottis 382

Eriocrania 80

Eriogaster 101, 316, 430

Eriopeltis 650

Eriophyes 346, 445

Eriopis 571

Eriopogodes 524

Eriopus 98

Eriosoma 232, 485, 555

Eristalinus 394

Eristalis 231, 264, 309, 394, 477, 500

Eristalodes 394

Ernobius 324

Ernodes 494

Erodius 47, 92, 100, 341

Erolia 588

Erotesis 494

Erthesina 506

Erymnis 712

Erynnis 19, 37, 101, 229, 315, $410,548,549$

Erythrapion 733

Erythromma 264, 396

Escharella 807

Escharina 807

Escharoides 807

Esolus 648, 744

Esperia 562

Esteparia 98 546,560

Esthiopte

Esthipterum 70

Ethmia 155, 191, 314, 370, 492,

$$
548,549,562
$$

Ethusa 710

Etiella 298, 548

Etmopterus 808

Etriptes 193

Eualus 619

Euatholus 723

Eublema 518

Eublemma 98, 524, 549, 560

Euborellia 592

Euboriella 345

Eubrachium 723, 817

Eubria 600

Eucallipterus 485

Eucalohister 723

Eucalymnatus 251, 650

Eucarazzia 485

Eucarta 427, 546, 560

Eucera 289, 320, 617, 654, 758, 780

Euceraphis 485

Euchalcia 560

Euchalcis 799

Eucharia 95, 560, 580

Euchloe 12, 61, 89, 93, 104, 113,

$135,157,190,315,316,358$,

$409,410,430,455,468,492$,

$526,545,548,549,629,659$, 660, 694

Euchloris 170, 316, 409, 492, 549

Euchorthippus 345, 614, 621

Euchorthipus 706

Euchroeus 127, 513

Euchromius 382, 386, 491

Eucinetus 600

Euclidia 560

Euclidimera 228

Euclismia 589

Eucnaemidophorus 382

Eucomys 364

Euconnus 39

Euconocephalus 506

Eucornuaspis 633

Eucosma 198, 229, 405, 562

Eucosmoides 149, 198

Eucosternum 506

Eucrostes 170, 228

Eucryptocerus 634

Eucyclops 537

Eucypris 537

Eudemis 198, 405

Eudia 45, 101, 371, 426, 545

Eudiplister 723

Eudistoma 801

Eudonia 137

Eudoria 155

Eudostomus 174

Eudoxyle 506

Eudryoctenes 290

Eudynerus 317

Eueremaeus 720

Eugnomus 129

Eugnorisma 95, 524, 549, 560

Eugnoristus 506

Eugnosta 405

Eugonia 16

Eugryllodes 345, 614, 706

Eukiefferiella 32, 532, 565, 785 


\begin{tabular}{|c|c|c|}
\hline Eumenis 229,671 & Euthales 492, 524 & Forelius 634 \\
\hline Eumerus 394, 500 & Euthera 812 & Forficesila 592 \\
\hline Eumichtis 61, 98, 492, 549, 560 & Euthyas 687 & Forficula $345,592,706$ \\
\hline Eumicrodynerus 584 & Euthystira 614 & Formica 18, 33, 129, 455, 457, \\
\hline Eumicromus 736 & Euthystra 614 & $466,506,543,553,645,656$, \\
\hline Eumigus 727 & Eutrichapion 733, 751 & $692,723,725,765,767,802$ \\
\hline Eunidia 290 & Eutrichomerus 103 & Formicaleo 22, 590 \\
\hline Euodynerus 317, 393, 584, 683, & Eutrichophilus 397 & Formicina 512 \\
\hline & Eutriptus 110, 723 & Formicoides 444 \\
\hline Euomphalia 768 & Euxanthis 229 & Formicomus 456 \\
\hline Euparypha 757 & Euxoa 61, 95, 189, 228, 229, 298, & Fosseremus 720 \\
\hline Eupelmus 636 & $316,427,468,506,524,560$ & Fournieria 587 \\
\hline Eupelogonus 817 & Euzonitis 138, 200 & Foveifera 149 \\
\hline Eupelops 419, 708, 720 & Euzophera 382, 549 & Frankliniella 615,771 \\
\hline Euphilippia 650 & Evallaspis 633 & Frauenfeldiella 232 \\
\hline Euphorus 163 & Evania 33, 129 & Frea 163,290 \\
\hline Euphya 228 & Everes 104, 229, 315, 410, 455, & Freadelpha 290 \\
\hline Euphydryas 104, 190, 194, 315, & 508,549 & Fremuthiella 733 \\
\hline $\begin{array}{l}358,386,508,526,548,549, \\
676,694\end{array}$ & $\begin{array}{l}\text { Evergestis } 155,229,382,492, \\
\quad 545,548,549\end{array}$ & $\begin{array}{l}\text { Friesea } 461,488,535,576,613 \\
\text { Friganea } 481\end{array}$ \\
\hline Euphyia 170, 229 & Evias 112 & Fringilla 588 \\
\hline Euphyllura 499 & Exaereta $90,190,549,643$ & Frondipora 807 \\
\hline Eupithecia 61, 155, 170, 191, & Exaesiopus 723 & Fulica 515,588 \\
\hline $211,228,298,371,386,468$, & Exanthidium 637 & Fulvoclysia 198 \\
\hline $492,545,548,549$ & Exapion 733,751 & Fumea 86, 157, 382, 545, 549 \\
\hline Eupizeuxis 524 & Exaraeta 61 & Funchalia 619 \\
\hline Euplagia 101, 229, 316, 386, & Exetastes 129, 274, 336, 704 & Fundaspis 251 \\
\hline $545,549,560$ & Eximia 290 & Furcaspis 251 \\
\hline Euplexia 98, 560 & Exocentrus 290 & Furchadiaspis 633 \\
\hline Eupoecilia 198, 405, 562 & Exochilum 302 & Gabrius 436 \\
\hline Euponera 725 & Exochomus 15, 110, 219, 518, & Gabunia 163 \\
\hline Euporus 290 & $571,582,603,680$ & Gadiculus 808 \\
\hline Euprepia $61,95,158,228,548$, & Exochus 263, 274, 319 & Galeria 548 \\
\hline $549,560,580$ & Exolytus 274, 336 & Galeruca 110, 219, 260, 456 \\
\hline Euproctis 45, 61, 98, 213, 347, & Exophila 98, 560 & Galerucella 277, 321 \\
\hline $384,406,509,548,677,560$ & Exorista 347 & Galgula $98,524,546,560$ \\
\hline Euprotapion 733 & Exosoma 681 & Galileja 786 \\
\hline Eupsila 560 & Exoteleia 405 & Galleria 191, 287, 371, 382, 549 \\
\hline Eupsilia 98 & Exothecus 577 & Gallinula 113,588 \\
\hline Euraspidiotus 251 & Eylais 687 & Gallus 397, 588 \\
\hline Eurhodope 382, 545 & Eyprepocnemis 345, 672, 706, & Galumna 419, 445, 488, 720 \\
\hline Eurhopalothrix 634 & 716 & Gambrus 336, 372 \\
\hline Eurodryas 712 & Fabrella 589 & Gambusia 264, 647 \\
\hline Europhilus 96 & Fabriciana 104, 194, 315, 316, & Gammarus 24, 744 \\
\hline Europs 110 & $358,548,549,676,694,712$ & Gardena 602 \\
\hline Eurranthis 113 & Facciolella 808 & Garrulus 397 \\
\hline Eurrhypara 382 & Fairmairia 650 & Gasteracantha 444 \\
\hline Euryasida 47 & Falagria $100,436,630$ & Gasterophilus 477 \\
\hline Eurycarabus 342 & Falco 397, 515, 534 & Gasteruption 263, 372 \\
\hline Eurydactilus 163 & Fallapion 733 & Gastrallus 435 \\
\hline Eurydema 125,208 & Falseuncaria 562 & Gastroidea 304, 701, 723 \\
\hline Eurygaster 125, 208 & Fannia $179,365,477,621$ & Gastropacha 101, 545, 549 \\
\hline Eurygnathus 110 & Feiderzetes 720 & Gastrophysa 456 \\
\hline Eurylabus 127, 336, 372 & Fermocelina 332 & Gasulliella 761 \\
\hline Eurylister 723 & Fenestrulina 807 & Gegenes 492, 548 \\
\hline Eurynassa 506 & Feronia 359 & Gehypochthonius 720 \\
\hline Eurynebria 38, 96 & Ferussacia 782 & Geina 382 \\
\hline Eurysops 290 & Fidonia 170 & Gelechia 46, 405 \\
\hline Eurytoma 636, 653 & Figites 577 & Gelis 175,319 \\
\hline Eusacornis 125 & Figularia 807 & Genadas 619 \\
\hline Eusarcoris 208 & Filalia 138 & Genarches 129, 336 \\
\hline Euschistus 506 & Filatima 405 & Genetta 397 \\
\hline Euserica 214 & Filicrisia 807 & Geniocerus 636 \\
\hline Euspilapteryx 357 & Filifera 800 & Gennadas 27 \\
\hline Euspilotus 701, 723 & Filippia 251, 650 & Gennaecus 397 \\
\hline Euspongia 800 & Filislata 463 & Geocoris 208 \\
\hline Euspongus 608 & Filistata 444, 503, 538, 689 & Geoica 485, 555 \\
\hline Eustenancistrocerus 584, 683 & Fiorinia 633 & Geomantis 706 \\
\hline Eustrophus 600 & Fita 589 & Geometra 298, 467 \\
\hline Eustrotia 98, 427 & Folsomia 461, 474, 488, 613 & Geomyza 500 \\
\hline Eusyllis 742 & Folsomides 461, 474, 805 & Geophilus 620 \\
\hline Eusymphagus 769 & Fonscolombia 666 & Georthocladius 785 \\
\hline Euteles 370 & Forbesaspis 251 & Georyssus 600 \\
\hline Eutelia 98, 316, 409, 548, 560 & Forcicula 344 & Geotomus 125, 208 \\
\hline Euterpia 95 & Forcipomyia 585, 632 & Geotrechus 360 \\
\hline Eutetranychus 346 & Forda 485,555 & Geotrogus 679 \\
\hline
\end{tabular}

Geotrupes 186, 237, 391, 511, $691,762,816$

Gerloubia 708, 720

Gerris 131, 208, 607, 682

Gibbaranea 688

Gibberifera 149

Gibbium 119

Gigantiops 634

Gilletteella 571

Gimnomerus 584

Glabrasida 47

Glabrobracon 656

Gladiovalva 405

Glaenocorisa 682

Glamyromyrmex 634

Glaphirus 438

Glaphyra 98, 524

Glareola 588

Glaresis 600

Glaucopsyche $19,104,136,190$ $195,228,229,315,316,386$ 409, 430, 455, 492, 545, 548, 549 Glenea 290

Glenosema 129

Gliphomerus 636

Gliptomorpha 193

Gliricola 397, 502

Glischrochilus 512

Glossina 17, 97, 163

Glossinella 262

Glossosoma 494

Glossotrophia 61, 170, 191, 468, 545,548

Gluphisia 90, 643

Glycyphagus 445

Glyphina 232

Glyphipterix 562

Glyphipteryx 370

Glyptotendipes 532, 565, 587

Gnamptogenys 634

Gnathaenia 290

Gnathocerus 47, 100, 119

Gnathoncus 723

Gnatophyllum 619

Gnopharmia 170

Gnophos 61, 170, 228, 229, 430,

$$
\text { 548, } 549
$$

Gnophota 110

Gnorimoschema 405

Gnorimus 88, 108, 119, 214

Gobius 647, 808

Goeldichironomus 752

Goera 494

Gohieria 445

Golgia 217

Goliathus 139, 506

Gomezmenoraspis 633

Gomezmenoria 382

Gomphocerus 614, 727, 737

Gomphocrates 405

Gomphus 647

Gonaspidiotus 251, 633

Goneplax 710

Gonepteris 410

Gonepteryx 12, 45, 61, 93, 102, $104,189,190,229,315,316$,

$386,409,430,468,526,545$

$546,548,549,659,660,694,712$

Gonimbrasina 506

Goniocotes 397, 588

Gonioctena 681

Goniodes 397, 473, 588

Goniomma 719, 725, 802

Gonioxybelus 365

Gonocephalum 47,92,96,110, $120,172,291,341,373,412$ 
Gonocerus 125, 208, 501

Gonolochus 635

Gonopteryx 358

Gonospileia 98

Gordius 46, 486

Gortyna 427, 524, 560

Gorytes 129, 222, 250, 471, 608

Gossyparia 251, 666

Graciella 163, 290

Gracilaria 163

Gracilia 92, 140

Gracilipalpus 61, 98, 560

Graellsia 2, 68, 80, 101, 134, 154

$182,191,194,252,316,334$

$371,386,406,407,409,424$

$430,453,455,468,508,509,522$ Graellsinus 374

Grammesia 560

Grammodes 61, 98, 157, 316, 409, 549, 560

Grammostethus 723

Grammotaulius 494

Grandia 193

Granopupa 757, 782

Graphocaecilius 589

Graphoderus 281

Grapholitha 405

Graphopsocus 589

Graphopterus 100

Graphosoma 125, 208

Graptodytes 122, 184, 529, 698

Graptolitha 98, 546, 549

Graptostethus 125

Gravenhorstia 302

Gravitarmata 149

Griphapex 290

Griselda 198, 405

Grocetia 588

Grocus 22

Grus 397, 588

Gryllodes 345

Gryllomorpha 345

Gryllotalpa $15,250,265,345$, $506,614,716$

Gryllus 345, 443, 506, 553, 614, $621,673,706,716,727,737$

Grynobius 43

Guadalgenus 707

Guerinia 666

Gueriniella 666

Guignotus 122, 161, 264, 698

Guilarovus 720

Guineodiadelia 290

Gunarus 47

Gymnancyla 382

Gymnaspis 633

Gymnetron 100, 751

Gymnochiromyia 756

Gymnocnemia 22

Gymnococcus 666

Gymnodamaeus 419, 720

Gymnomerus 393

Gymnomyrmex 634

Gymnopleurus 139, 186, 237, 691

Gymnoscelis 61, 170, 228, 298 ,

$316,430,548$

Gymnosoma 812

Gynamica 506

Gynandophthalma 260

Gynandromorphus 63

Gynandrophthalma 219, 258,

266, 276, 411, 697

Gypaetus 397, 515, 573

Gyps 515, 573

Gypsonoma 198, 229, 405

Gypsonomoides 149, 198, 405
Gyranusa 364

Gyrinus 15, 77, 122, 161, 529, 698

Gyrocampa 193, 337

Gyrophaena 436

Gyropus 397, 502

Habraeus 723

Habrocryptus 274

Habrocytus 636

Habrolepis 364

Habrolepsis 215

Habrophlebia 744

Habropoda 289

Habrosyne 90, 405

Hada 95, 298, 560

Hadena 95, 228, 298, 302, 406, 409, 427, 492, 508, 524, 545, 546,560

Hadjina 98, 386, 560

Hadrobregmus 435

Hadrocarabus 62, 261, 631, 804

Hadrodactylus 129

Hadromanus 302

Haemabarus 502

Haemagogus 4, 754

Haemantopus 588

Haemassia 98

Haematobia 262, 365

Haematobosca 262

Haematopota 365, 417, 459, 554, 586

Haemerosia 560

Haemonia 257

Haemorrhagia 8

Haenydra 648, 699

Haetaerius 817

Halacritus 456, 723

Halammobia 96, 120, 413

Halarachne 445

Halesus 494

Haliaetus 573, 588

Haliastur 573

Haliastus 588

Halictus 58, 289, 531, 617, 621, $654,713,722,758,780$

Haliplus 122, 161, 529, 600, 698

Halizia 680

Halobatrachus 808

Halocladius 532, 565

Halocynthia 801

Halomachilis 542

Haloxantha 110

Haltica 260, 304

Halticoptera 577

Halyzia 232, 582, 603, 649

Hamamelistes 485

Hamartema 382

Hamearis 104

Hammomyia 365

Hanumanus 748

Hapheniastus 290

Haplidia 214, 248, 662

Haplochthonius 445, 720, 760

Haplocnemus 219

Haplodiplosis 519

Haplophilus 620

Haplopoma 807

Haploprocta 208, 501

Haplothrips 216, 638

Haplozetes 419

Haptoderus 62, 173, 530

Haptoncus 551

Haritala 228, 316, 382

Harmodia 61, 95, 560

Harmonia 219, 582, 649, 680

Harnischia 587

Harpactor 176
Harpadispar 382

Harpagochares 602

Harpagophana 95, 524, 560

Harpalus 38, 62, 92, 96, 140,

220, 412, 601, 631, 700

Harpapion 733

Harpella 370

Harpobittacus 543

Harpya 90, 190, 549

Harpyia 545, 563, 643

Hastula 198, 405, 545

Hayesomyia 785

Hayhurstia 485, 555

Hebecnema 365

Hebrus 682

Hecphora 290

Hedobia 324, 435

Hedya 198, 405, 549, 562

Hedychridium 458, 713

Hedychrum 129, 440, 458, 572, 713

Hegeter 110, 140, 291

Heinemannia 370

Heleniella 532

Heleomyza 756

Heleonomus 588

Helianthemapion 733

Helicella 757, 768

Helichus 600

Helicigona 768

Helicoconis 591, 618, 806

Helicodonta 761, 768

Heliconius 543

Helicopsis 768

Helicopsyche 494

Heliococcus 251, 633, 666

Heliococonis 686

Heliocopris 506, 762

Heliocorisa 682

Helioctamenus 340, 496

Heliodines 370

Heliopathes 120, 230, 341, 412

Heliophilus 3, 96

Heliophobus 95, 560

Heliotaurus 681

Heliothea 170, 492, 545

Heliothela 382

Heliothis 44, 95, 211, 228, 229

$315,371,384,386,468,545$, $558,560,711$

Helix 729, 757, 761, 768, 782

Hellula 382, 409, 549

Helochares 77, 456, 529, 648

Helodrilus 781

Helopeltis 163

Helophilis 309

Helophilus 529

Helophorus 100, 456

Helops 96

Helorus 335

Hemaris 101, 316, 431

Hemerobius 22, 589, 591, 618, 686, 747

Hemerophila 155, 170, 468, 492, 545

Hemianax 396

Hemiberlesia 163, 251, 633

Hemileius 419, 720

Hemilucilia 732

Hemimelaena 282, 707

Hemineura 589

Hemipodius 113

Hemipterochilus 584

Hemisemidalis 686, 806

Hemistola 170

Hemiteles 215, 336, 577

Hemithaea 170

Hemitrichapion 733

Henicocerus 648, 699

Henicopus 374

Henosepilachna 582,603

Henrotius 360

Heodes 712

Hepialus 80, 158, 357, 386, 548

Hepiopelmus 336

Heptastruma 634

Heptaulacus 600, 773

Herculia 382, 430, 549

Hercynodes 382, 492, 549

Heriades 289, 320, 637, 758, 780

Hericia 445

Heringia 394

Heringita 187, 370, 387, 406

Hermaeophaga 304

Hermannia 419

Hermanniella 419

Hermetia 477, 564

Herminia 98, 524, 549, 560

Herpestes 113

Herpetocypris 537

Herpisticus 110

Herrichia 370

Herse 76, 101, 236, 386, 430, 480, 549

Hesperia 19, 37, 61, 101, 134,

189, 209, 229, 315, 410, 506, 548, 549, 712

Hesperocorixa 682

Hesperophanes 71, 120, 183, 746

Hesthesis 450

Hetaerius 723, 817

Heteracris 345, 672, 706, 716

Heterhelus 512

Heterobostrychus 239, 484

Heterocerus 100, 600

Heterocola 635

Heteroconis 806

Heterocypris 537

Heterodera 420

Heterogaster 125, 208

Heterognathus 723

Heterographis 382

Heterogynis 86, 228, 332, 492

Heterogynnis 37

Heterolepinotus 589

Heteromma 98

eteromurus 461, 516, 535, 576,

613

Heteronympha 759

Heterophysa 427, 560

Heteropoda 463, 503, 538

Heteroponera 634

Heteropsilus 163

Heteropterina 365

Heteropterus 101, 191, 315, 409, 410

Heterostemma 110

Heterostigma 801 


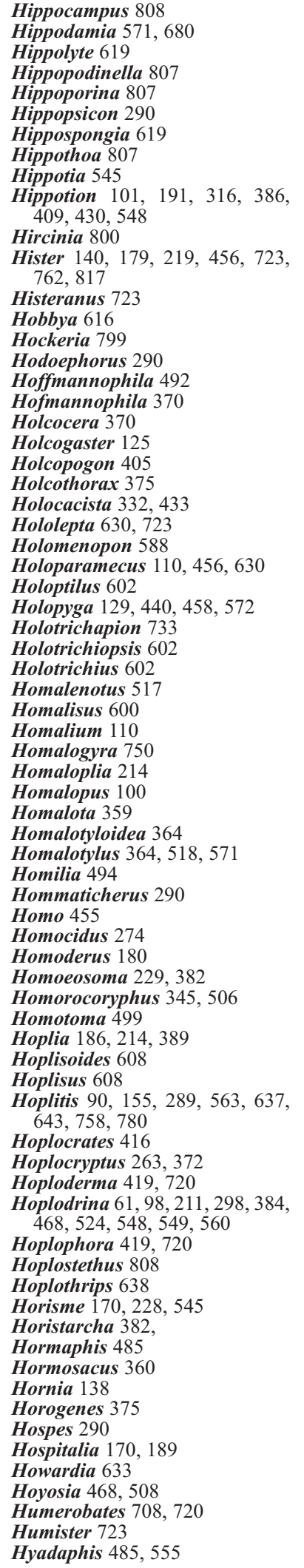

Hyagnis 290

Hyalina 406, 729, 761

Hyalinia 729

Hyalinobatrachium 787

Hyalopterus 232, 485, 555, 571

Hybalus 600

Hyblea 506

Hyboclonius 290

Hyboma 524

Hybomitra 459, 554, 586

Hybosorus 600

Hybunca 290

Hydaticus 161

Hydrachna 687

Hydraecia 189, 211, 400, 409

$427,446,524,545,560$

Hydraena 648, 699

Hydrelia 467

Hydriomena 170, 467

Hydrobilus 699

Hydrobius 77, 456

Hydrochus 600, 648

Hydrodroma 687

Hydroecia 98, 189, 549

Hydrometra 208, 682

Hydrophilus 15, 456, 529, 603, 648, 699

Hydroporus 77, 122, 161, 184 281, 391, 529, 698

Hydropsyche 494, 606, 744

Hydroptila 494

Hydroscapha 600

Hydrotaea 179, 459

Hydrous 77, 264, 506, 529, 648

Hydrovatus 122, 161

Hydrozetes 419, 720

Hydryphantes 687

Hygophum 808

Hygrobates 687

Hygrobia 122, 600, 698

Hygrocarabus 96, 261

Hygromia 768

Hygrotus 161

Hylaeus 289, 320, 439, 499, 608 , $722,758,780$

Hylastes 373, 456

Hylecoetus 180, 600

Hyles 492

Hyllisia 290

Hylobitelus 751

Hylobius 321

Hyloicus 76, 101, 302, 386, 545

Hylomyrma 634

Hylophila 189

Hylotrupes 71, 92, 269, 746

Hylurgus 373, 456, 512, 723

Hymemya 365

Hymenalia 456

Hymenia 299, 382

Hymenochelus 214

Hymenodes 529, 648

Hymenoplia 186, 219

Hymeroplia 214

Hypatim 290

Hypebaeus 437

Hypena 61, 98, 228, 409, 524,

$545,548,549,560$

Hypenodes 98

Hypera 681

Hyperaea 812

Hyperaspis 232, 582, 603, 680

Hypercallia 370

Hyperchalcidia 799

Hyperlais 382

Hyperomyzus 485, 555, 745

Hyphantria 310
Hyphilare 549

Hyphilarea 95

Hyphoraia

Hyphydrus 122, 161, 698

Hypnoidus 391

Hypoborus 120, 456

Hypocacculus 701, 723, 817

Hypocaccus 456, 723

Hypocassida 681

Hypochalcia 382

Hypochthoniella 419, 720

Hypochthonius 419, 720

Hypoclinea 634

Hypocoprus 600

Hypodamaeus 720

Hypoderma 506$$
535,735
$$

Hypogeobium 67, 75

Hypogryphia 382

Hypogymna 98, 213

Hypolais 113

Hyponephele 16, 315, 316, 386 , $549,629,660,694,712$

Hyponomeuta 45, 229, 375

Hypophloeus 47, 373

Hypoponera 634, 715, 725

Hypopta 61, 86

Hypopus 445, 531

Hyposoter 711

Hypotia 382, 430

Hyppa 560

Hypponephele 545

Hyppotion 56

Hypsenor 817

Hypsicera 263

Hypsopygia 228, 382, 430, 549

Hypsosinga 688

Hypsotropa 382

Hyptiotes 463

Hysterophora 405

Hysteropterum 471

Hysterosia 198, 405

Ibalia 372

Iberococcus 666

Iberodorcadion 697, 746, 764

Iberoppia 720

Iberus 768

Iceranthidium 637

Iceria 78

Icerya 119, 163, 498, 666

Ichenumon 336

Ichneumon 129, 274, 302, 372, 400, 446, 499, 636, 667

Ichneumonitocris 290

Ictoplectis 376

Idaea 545

Idiomicromus 736

Idiopterus 555

Ifnidius 363

Ildibaha 444

Ildobates 360, 568

Ilia 710

Iliocypris 537

Ilybius 122

Ilyocoris 682

Inachis 407, 430, 526, 676, 694

Inachus 710

Incurvaria 357

Infurcitinea 332

Insculptoppia 720

Insulasida 47

Insulaspis 633

Inuus 113

Invreia 799
Iodis 170

Iolana 386, 430, 508, 712

Ionthodes 290

Iphiclides 104, 315，316，358,

$386,406,430,492,526,545$,

$548,549,629,712$

Iphigena 757

Ipidia 512, 570

Ipimorpha 98, 560

Ips 141, 551, 570, 723

Ircinia 800

Iridomyrmex 457, 634, 765

Iris 345,706

Ischioceras 722

Ischiodon 500

Ischnomera 306

Ischnonyctes 602
Ischnopterapion 733

Ischnoscia 332

Ischnura 264, 396, 647

Ischnus 263, 274

Ischyropsalis 487, 517

Isidora 264

Isocerus 47, 290

Isocolus 653, 668

Isodromus 364

Isogenus 282

Isognomostoma 768

Isolomalus 723

Isoneurothrips 615

Isoperla 282, 707

Isophrictis 405

Isoscelipterum 22

Isotoma 461, 474, 488,535,576, 613,805

Isotomiella 461, 488, 535, 613

Isotomina 461, 488, 613

Isotomodes 461, 535, 613, 778, 805

Isotomurus 461, 535, 576

Isotomus 119

Isotrias 198, 405, 562

Issoria 16, 68, 104, 134, 136,

$194,236,315,358,468,526$

$548,549,660,676,694,712$

Isturgia 170

Isurgus 129, 512, 635

Italochrysa 590, 591, 618, 686

Itame 170, 228, 430, 492, 545

Ithomia 450

Ithytrichia 494

Ittrion 723

Ixapion 733

Ixias 733

Ixodes 445

Jadera 501

Jalla 208

Jaminia 757, 782

Jodia 560

Johannsenomyia 632

Jordanoglenea 290

Jordanoleiopus 290

Josandreva 22, 395, 686

Judolia 88

Julodis 100, 219, 810

Kakatoe 397

Kakothrips 615

Kalcapion 733

Kalkapion 733

Kaltembachiella 485

Kaltenbachiella 555

Kanetisa 229

Katamenes 393, 671, 683

Kedirinus 748

Kermes 251, 650

Kermococcus 251, 650 


\begin{tabular}{|c|c|c|c|}
\hline Kessleria 215, 370 & Langelandia 103 & Lepidorhombus 808 & Leucoptera 332, 387 \\
\hline Kiefferulus 587 & Lanius 397 & Lepidosaphes $215,216,251,504$, & Leucorrhinia 264 \\
\hline Kissingeria 733 & Laodamia 382 & 633 & Leucosomos 92 \\
\hline Kissister 63, 723, 817 & Laothoe 101, 492, 545, 549 & Lepidostoma 494 & Leucospis 249 \\
\hline Klimeschiopsis 405 & Laotris 193, 337 & Lepidotrigla 808 & Leuctra $282,606,707,788,811$ \\
\hline Klugia 19 & Laparocerus $107,110,140$ & Lepinotus 589 & Leydigia 537 \\
\hline Knemidocoptes 445 & Laphygma $61,98,298,443,549$, & Lepisma 543 & Liacarus 720 \\
\hline Kobeltia 775 & 560 & Lepralia 807 & Libelloides 591, 618, 686 \\
\hline Korscheltellus 158, 357, 406, 492 & Lapismachilis 575 & Leptacinus 100 & Libellula 264 \\
\hline Krenopsectra 587 & Lapithes 589 & Leptaleu 456 & Libethra 506 \\
\hline Krenosmittia 785 & Lapton 704 & Leptanilla 457, 634 & Libythea $19,76,104,157,315$, \\
\hline Kribioxenus 587 & Larcasia 494 & Leptanilloides 634 & $386,409,548$ \\
\hline Krizousacorixa 506 & Larentia 113, 170, 298, 467 & Leptapion 733 & Lichenophagus 107, 110, 140 \\
\hline Krombeinella 722 & Laria 213,226 & Leptestheria 536 & Lichenophanes 108, 239, 484 \\
\hline Kudakrumia 722 & Larinioides 688 & Leptidea 12, 104, 209, 229, 315, & Lichenopora 807 \\
\hline Kyra 382 & Larinus $110,219,391,506$ & $358,386,410,430,455,526$ & Lichenovora 332 \\
\hline Labia 344 & Larra $222,250,651,684,685$, & $548,549,659,694$ & Lichtensia 650 \\
\hline Labidostomis 238, 258, 260, 266, & 713 & Leptidia 37,545 & Licinopsis 110 \\
\hline $276,304,681$ & Larsia 785 & Leptinotarsa $9,44,71,78,88$ & Licinus $38,62,92,96,325,631$, \\
\hline Labidura 344, 412, 592, 706 & Larus 397, 588 & $119,121,299,409,456$ & 700 \\
\hline Labidus 634 & Lasia 603 & Leptinus 600 & Licneremaeus 720 \\
\hline Laboulbenia 666 & Lasiocampa $41,61,89,101,127$, & Leptoceraea 501 & Licnobelba 720 \\
\hline Labroides 544 & $229,298,302,314,372,410$ & Leptocerca 477 & Licnodamaeus 419, 720 \\
\hline Labrorychus 302 & $546,548,549$ & Leptocerea 501 & Licnoliodes 419 \\
\hline Lacanobia 468 & Lasiocephala 494 & Leptocerus 494 & Lidar 22 \\
\hline Laccobius 529, 648, 699 & Lasiocoris 125 & Leptochilus 317, 393, 584, 683 & Liebstadia 708, 720 \\
\hline Laccophilus 77, 117, 122, 161, & Lasioderma 44, 324, 435 & Leptochironomus 587 & Lieurus 705 \\
\hline 698 & Lasioglossum 289, 654, 722, 758, & Leptocircu & Ligdia 170 \\
\hline Lacerta $113,721,738,739$ & 780 & Leptoclonius 290 & Ligephila 492 \\
\hline Lachanaea 278 & Lasiommata 546, 629, 660, 694, & Leptoconops 632 & Lilioceris 456 \\
\hline Lachesilla 589 & 712 & Leptocoris & Limacocarenus 501 \\
\hline Lachnaea 221, 238, 276, 277, & Lasiopezus 290 & Leptocorisa 506 & Limantria 41, 56, 61, 80 \\
\hline & Lasiophanes 634 & Leptocycl & $\operatorname{Limax} 757,775,776$ \\
\hline Lachnea 258, 260, 266 & Lasius $18,33,120,129,217,455$, & Leptodema 602 & Limenitis 16, 37, 104, 195, 229, \\
\hline Lachnomyrmex 634 & $457,466,543,570,656,715$ & Leptogenys 634 & $315,316,358,386,409,410$, \\
\hline Lachnosterna 506 & $723,725,765,802$ & Leptohylemyia 519 & $430,453,526,548,549,660$, \\
\hline Lachnus 232, 485, 555, 692 & Lasperyia 524, 560 & Leptomastidea 364 & 694,712 \\
\hline Lacinius 517 & Laspeyresia 198, 224, 228, 405, & Leptomastix 364 & Limnaeum 322 \\
\hline Lacmobothrion 397 & $519,545,548,549,551,562$ & Leptomer & Limnebius 648, 699 \\
\hline Lacon 630 & Laspeyria 98 & Leptomyrmex 506 & Limnephilus 494 \\
\hline Laelia $98,213,560$ & Lathridius 87 & Leptopalpus 138 & Limneria 129, 336 \\
\hline Laemobothrion $515,573,588$ & Lathriopyga 461, 576 & Leptophyes & Limnerium 375 \\
\hline Laemophloeus 110, 120, 308, & Lathrobium 436 & Leptopternis 345 & Limnesia 687 \\
\hline 456,630 & Lathrops 308 & Leptopus & Limnichus 600 \\
\hline Laemostenus 38, 119 & Lathyrophthalmus 394, 621 & Leptorches & Limnius 744 \\
\hline Laemosthenes 96, 140 & Latilamellobates 720 & Leptosia 1 & Limnochares 687 \\
\hline Laemothrion 397 & Latisternum 290 & Leptothorax $129,457,634,715$, & Limnochironomus 587 \\
\hline $\begin{array}{l}\text { Laeosopis } 19,104,315,430,492 \\
\quad 548\end{array}$ & $\begin{array}{l}\text { Latrodectus } 26,487,503,538, \\
755\end{array}$ & $\begin{array}{l}719,725,767,802 \\
\text { Leptotyphlus } 7\end{array}$ & $\begin{array}{l}\text { Limnophora } 606 \\
\text { Limnophyes } 532,785\end{array}$ \\
\hline Laesopis 61, 228 & Lauria 782 & Leptura 88, 107, 391, 407, 569 & Limnoporus 682 \\
\hline Laetilia 382 & Lauxania 365 & Leptus 810 & Limnoxenus 456 \\
\hline Lagarotus 336 & Leachia 666 & Leptynia 345 & Limnozetes 419, 720 \\
\hline Lagopoecus 473 & Lebertia 687 & Lepus 113, 206 & Limosa 588 \\
\hline Lagopus 473,588 & Lebia 38, 110, 391 & Lepyrus 391 & Limothrips 609 \\
\hline Lagorina 138 & Lecanium 163, 251, 364, 650, & Leskia 812 & Lindenius $608,651,722$ \\
\hline Lagria 323, 456, 600 & 666 & Lesopsocus & Lindingaspis 6 \\
\hline Lagrida 290 & Lecanodiaspis 251, 650 & Less & Lindorus 219, 498, 680 \\
\hline Lamellocossus 300, 405 & Lecanopsis 650 & 486,503 & Lineocrypticu \\
\hline Lamia $290,506,746$ & Lecithocera 405 & Lest & Linepithema \\
\hline Laminaria 807 & Lehmannia 757 & Le: & Linnaemyia 812 \\
\hline Laminioptes 445 & Leichenum 47, 120, 341, 413 & Lestiphor & Liocarcinus 710 \\
\hline Lamoria 382 & Leiobunum 487, 517 & Lethierrya 602 & Liochthonius 419, 720, 760 \\
\hline Lampetia 394 & Leiomyrmosa 722 & Lethocerus 506 & Liocnemis 96 \\
\hline Lampides $19,61,89,104,134$, & Leionotoxenos 318 & Leucania 95, 299, 427, 524, 548, & Liocola 214 \\
\hline $228,229,315,409,410,430$ & Leionotus 318 & 549 & Liodes 419, 708, 720 \\
\hline $468,509,548,549,629,656$ & Leiopelta 634 & Leucaspis 633 & Liogaster 394 \\
\hline 660 & Leioptilus 382 & Leucochlaena 61, 98, 316, 548, & Liogryllus 506 \\
\hline Lampra 630 & Leiopus 88, 290 & 549,560 & Liometopum 634, 765 \\
\hline Lamprias 62 & Leistus 38, 325, 631 & Leucochloe 12 & Liomutilla 442, 531 \\
\hline Lamprocrypticus & Lema 71 & Leucochroa 757 & Lionychus 38, 96, 359 \\
\hline Lamprodema 125 & Lemonia $61,101,548$ & Leucodonta 643 & Liopterus 391 \\
\hline Lampromyia 177, 500 & Lepidapion 733, 751 & Leucoma 98, 213, 549, 560 & Liopus 290 \\
\hline Lampronia 357 & Lepidiota 506 & Leuconea 12 & Liorhyssus $125,208,501$ \\
\hline Lampropteryx 170 & Lepidocyrtinus 4 & Leucopholis 506 & Liosomaphis 555 \\
\hline Lamprote & Lepidocyrtus 461, 474, & Leucopis 232 & Liotheum 502, 515, 573 \\
\hline Lampyris 226, 681 & Lepidopus 808 & Leucopsis 127 & Lipaphis 485 \\
\hline
\end{tabular}


Lipara 193

Liparis 49, 213, 302

Liparthrum 110

Lipaspis 140

Lipeurus 397, 534, 588, 705

Liposcelis 589

Lipura 461

Liriomiza 337

Liriomyza 193

Liris 621, 651, 684, 685

Liryomiza 13

Lissoclinum 801

Lissonota 336

Listrodromus 263

Lita 46, 405

Litargops 110

Litargus 456

Lithacodia 98, 560

Lithax 494

Lithina 61, 170, 228, 545, 549

Lithobius 620

Lithocolletis 168, 357, 581

Lithophane 61, 98, 427, 546, 560

Lithophilus 110, 680

Lithosia 61, 95, 189, 298, 545, $546,549,560,580$

Lithostege 61, 155, 170

Lithotanytarsus 703

Lithurgus 289, 637

Litocharis 140

Litomeces 290

Litopus 290

Litororus 230

Litosomoides 445

Lixodessa 405

Lixus 219, 391, 399, 420, 446, 681,75

Lobesia 198, 405, 548

Lobonyx 456

Lobophora 170, 467

Loboptera 706

Loborhynchapion 733

Lochmaea 304

Locusta 207, 284, 345, 443, 506,

$611,614,626,706,716,727,737$

Locustana 506

Lodiana 382, 386

Loensia 589

Logicornius 13

Lohmannia 419, 720

Lomaspilis 170, 211

Lomechusa 71

Lomographa 61, 170, 548, 549

Lonchaea 179, 365

Longicornutia 405

Longitarsus 140, 219, 238, 260, 277, 304

Longiunguis 571

Lophius 808

Lophocateres 119

Lophopteryx 90

Lophoterges 95, 560

Lophura 588

Loricaster 600

Lorrya 346

Loweia 19

Loxocnemis 501

Loxostege 382, 420, 468, 545, 549

Loxotropa 163

Lozotaenia 198, 405, 468

Lozotaeniodes 198, 405, 468, 545

Lucanus 88, 214

Lucasius 674

Lucifer 619

Lucilia 78, 179, 222, 365, 477

Lucoppia 720
Ludius 88

Luffia 86, 382

Lunaceps 588

Lundella 634

Luperina 95, 400, 524, 560

Luperus 219, 277, 279, 304, 528

Lusitanococcus 666

Luteva 602

Lutevopsis 602

Luzonoptinus 748

Lybithea 316

Lycaeides 209, 712

Lycaena 19, 37, 61, 89, 93, 102

$104,113,134,135,137,153$,

$158,189,194,195,228,229$

$315,316,358,409,410,430$

$455,468,492,548,549,629$, 660,712

Lycia 61, 170, 401, 467

Lycoperdina 217

Lycophotia 95, 546, 560

Lycosa 26, 113, 444, 503, 538, 755

Lyctocoris 125

Lyctus 180, 321, 343, 551, 630

Lyda 476

Lydus 1380.

Lygaesoma 125, 208

Lygaeus 501

Lygephila 560

Lygistopterus 600

Lygris 170, 467

Lygus 420, 558

Lymantria 45, 61, 73, 76, 98, 134

$157,213,302,321,333,347$

$376,406,508,509,549,560$

Lymexylon 180, 600

Lymnaea 264, 744

Lymnophyes 565

Lyncometra 549

Lynx 113

Lyonetia 332, 548

Lype 494

Lyperosia 262

Lyphia 47, 120, 484

Lyreus 103

Lyriomiza 13

Lyristes 151, 404

Lyrurus 473

Lysandra 156, 209, 229, 430 $549,629,660$

Lysiphlebus 232, 670, 798

Lysmata 619

Lyssandra 712

Lythria 61, 170, 371, 492, 548, 549

Lytta 15, 28, 138, 288

Lyttonyx 100, 138

Macaria 170, 228, 548, 549

Maccacus 445

Maccevethus 208, 501

Macdunnoughia 560

Machilinus 575

Machilis 575, 603

Machuella 720

Macrocera 289

Macrocheles 445

Macrochia 290

Macrocoma 100

Macrocyclops 537

Macrodiplax 264

Macrodontia 506

Macroglosa 41

Macroglossa 56

Macroglossum 8, 45, 61, 101, 229

$315,430,480,492,545,548,549$

Macrogyropus 502

Macrolenes 260

Macrolister 456, 723

$591,618,686$

Macroneura 636

Macronychia 365

Macropelopia 24

Macropeza 632, 664

Macropipus 619

Macropis 289

Macroplax 125, 208

Macropodia 710

Macroptylum 632, 664

Macroramphosus 808

Macrorhynchapion 733

Macrosandalus 602

Macroscytus 125, 208

Macrosila 506

Macrosiphon 232

Megalomerium 208

571, 745

Macrosiphum 485, 499, 555

Macrotermes 506

Macrothorax 261, 804

Macrothylacia 101, 549

Macrotoma 163, 290, 461, 506

Macrotrichopherus 363

Mateuina 100

Maurolicus 808

Maxillaria 382

Mayetia 27

Mecolenus 733

Mecosaspis 290

Mecyna 382, 545, 549

Medioppia 720

Medon 100

Meesia 332

Megabunus 517

Megacephala 322, 600

Megachile 138, 183,200, 268, 289, 637, 758, 780

Megaliche 439

Megalomus 22, 590, 686

Megalomyrmex 634

Megalona 560

Megalothorax 461, 535

Meganephria 61, 95, 560

Megaphisa 299

Megaselia 128, 347

Maculinea 104, 156, 157, 191, Megasis 382

195, 315, 316, 386, 409, 433, Megasoma 139, 506 455

Madopa 560

Magasternum 762

Magdalis 751

Maheoptinus 748

Megasternum 456

Megathrips 638

Megatracheloides 733, 772

Megatrupes 691

Malachius

512, 697

Malacogaster 110, 600

Malaconothrus 419, 720

Malacosoma 45, 56, 61, 101 197, 302, 333, 371, 508, 509 $545,548,549,583$

Maladema 128

Maladera 214

Mallada 686

Mallodon 290, 506

Malthinus 140

Malthodes 219

Malvapion 733

Mamestra 191, 316, 492, 524, $545,548,560$

Mangora 688

Mania 98, 549

Megistopus 22, 686

Megistopus
Megopis 88

Megoura 485, 555

Meladema 48, 77, 122, 161, 264, 698

Melambia 100

Melanagromyza 337

Melanaphis 485, 555

Melanapion 733

Melanargia $12,16,61,68,102$ $113,196,236,315,316,386$, 409, 410, 430, 468, 492, 545,

$548,549,629,660,694,712$

Melanargyia 37

Melanaster 506

Melandrya 88

Melanimon 172, 240

Melanitta 588

Maniola 104, 236, 315, 386, 409 $410,430,492,545,548,549$, $629,660,694,712,759$

Mannia 61

Mansonia 754

Mantis 35, 318, 345, 543, 706

Mantisoma 602

Mantispa 486, 618, 686

Mantura 277

Manuelia 552

Marasmarcha 229, 382

Marcenendius 589

Marchaliella 251

Margarinotus 701, 723

Margarodes 666

Margaronia 548

Marpissa 486

Marthamea 282, 707

Martyrhilda 370

Marumba 101, 191, 430, 548

Masonia 86, 382

Masoreus 38, 100, 110, 220

Masticocerus 602

Melanochrus 110, 291

Melanocoma 110

Melanocoryphus 125

Melanogromyza 193

Melanogryllus 614

Melanophthalma 630

Melanoplus 203, 207

Melanopolia 290

Melanopsis 647

Melanostoma 231, 309, 394, 500

Melanotus 88, 321, 630, 681

Melanthia 170, 386

Melasina 86, 382

Melasis 88, 321

Melasmana 110

Melasoma 260, 456

Meleageria 209

Meleagris 397

Melecta 129, 289, 320, 617 


\begin{tabular}{|c|c|c|c|}
\hline Melitaea 16, 37, 61, 64, 89, 102, & Metabletus 38, 110, 140 & Micropus 502 & Monostira 208 \\
\hline $104,113,153,195,228,229$, & Metachorudina 461 & Microsetia 405 & Monotoma 308, 456, 630 \\
\hline $315,316,358,409,410,430$, & Metachrostis 560 & Micrositus 3, 47, 92, 230, 341, & Monotropus 214, 248, 259, 662 \\
\hline $468,492,507,509,526,545$, & Metacoelus 372 & $373,412,697$ & Monoxenus 290 \\
\hline $546,548,549,558,660,676$, & Metacrambus 382, 386 & Microsphaecia 86 & Moralesia 100 \\
\hline 694,712 & Metallina 62, 96, 631 & Microstega 382 & Mordella 456, 630, 681 \\
\hline Melithreptus 500 & Metalype 494 & Microtendipes 42, 532, 565, 587, & Morellia 459 \\
\hline Melitta 289 & Metamhyrra 649 & 703,785 & Morica 412 \\
\hline Melittobia 365 & Matanarsia 405 & Microterys 364 & Morimus $88,630,764$ \\
\hline Melitturga 289 & Metaphycus 518 & Microthrix 382 & Moritziella 720 \\
\hline Meliturga 320 & Metapion 733 & Microtinea 332 & Mormo 492, 560 \\
\hline Mellicta 104, 150, 195, 315, 358, & Metaplatybunus 517 & Microtritia 720 & Mormonia 98, 191, 545 \\
\hline 549,712 & Metaporus 161, 698 & Microtyphlus 199, 360 & Morophaga 332 \\
\hline Mellinus $129,608,621$ & Metapterus 602 & Microus 119 & Morter 22, 396 \\
\hline Meloe 15, 28, 58, 100, 138, 140, & Metasia 228, 229, 316, 382, 548 & Microvelia 682 & Morysmula 722 \\
\hline 456,681 & Metatetranychus 346 & Microxeromagna 768, 782 & Moureia 524, 546, 560 \\
\hline Meloidogyne 420 & Metatrichapion 733 & Microzetorchestes 720 & Mulcticola 534 \\
\hline Melolontha 15, 31, 69, 214, 248, & Metaxmeste 382 & Microzoum 240 & Mullus 808 \\
\hline $321,420,438,446,506,603,662$ & Meteorus 689,711 & Micrurapteryx 357 & Multioppia 720 \\
\hline Melophorus 506 & Metholcus 324 & Mideopsis 687 & Multoribates 720 \\
\hline Melyrosoma 110 & Metophthalmus 630 & Migneauxia 456 & Murcia 720 \\
\hline Membraniporella 807 & Metopia 365 & Milax 757 & Murosternum 290 \\
\hline Memythrus 225 & Metopoceras $61,95,155,316$, & Milesia 231, 394 & Musaria 569 \\
\hline Menacanthus $397,534,588$ & $409,492,524,545,546,549$ & Milliereia 370 & Musca $54,179,222,262,318$, \\
\hline Menaccarus 208 & 560 & Milthochrista 560,580 & $365,459,477,500,514,577$ \\
\hline Mendesia 357 & Metoponorthus 674, 675 & Miltochrista 95, 429 & $610,611,626,683$ \\
\hline Mendizabalia 697 & Metopoplax 208 & Miltogramma 365 & Muscina $179,222,477$ \\
\hline Mendizabalina 697 & Metriochroa 357 & Milvus $397,515,534,588$ & Mutilla 416, 442, 489, 608, 722 \\
\hline Mengoana 768 & Metriocnemus 32, 46, 532, 565 & Mimas 76, 101, 407 & Mycalesis 558 \\
\hline Menopon 397, 534, 588 & Metylophorus 589 & Mimesa 129, 250, 392, 652, 684 & Mycetaea 119, 217 \\
\hline Meporus 290 & Metzneria 562 & Mimofrea 290 & Mycetarotes 634 \\
\hline Mercetaspis 633 & Mezium 110 & Mimumesa 652 & Mycetina 217 \\
\hline Mergellus 588 & Miana $98,427,430,560$ & Minoa $170,545,548$ & Mycetoma 600 \\
\hline Mergus 588 & Miarus 751 & Minois 104, 315, 386 & Mycetophagus 88, 630 \\
\hline Meridiophila 382 & Micaria 444 & Mintho 812 & Mycetophylax 634 \\
\hline Meristis 98 & Micilus 600 & Minucia $61,98,316,545,549,560$ & Mycetosoritis 634 \\
\hline Merites 193 & Micipsa 100 & Minunthozetes 720 & Mycobates 708,720 \\
\hline Merluccius 808 & Micra 427 & Miopristis 260, 266 & Mycocepurus 634 \\
\hline Mermis 46, 486, 571 & Micragona 506 & Mirificarma 405, 545 & Mycterothrips 615 \\
\hline Merocoris 501 & Micrambe 63, 456 & Mirrha 680 & Mycterus 600 \\
\hline Meromenopon 588 & Micranurida 461, 535 & Miscophus 129, 250, 651, 684 & Myctophum 808 \\
\hline Merophysis 630 & Micrapta 723 & Miselia 95, 155, 549 & Myelois 155, 235, 316, 382, 492, \\
\hline Meropoecus 534, 588 & Micrasema 494 & Misetus 263 & 549 \\
\hline Merops $113,397,534,588,701$, & Micrasemodes 494 & Misolampus 47, 114 & Myelophilus 141 \\
\hline 723 & Micraspis 456 & neng 444 & opa 309, 394, 500 \\
\hline Meropsiella 534 & Micrathena 444 & Misumenops 538 & Myinodes 170 \\
\hline Mesachorutes 461 & Micrelytra 208, 501 & Mithymna 546 & Myiobia 204 \\
\hline Mesapamea 560 & Micreremus 708, 720 & Mitostoma 517 & Mylabris 58, 100, 138, 219, 456 \\
\hline Mesaphorura 488, 535, 734, 743 & Microchironomus 587 & Mityloccocus 346 & Myodiadelia 290 \\
\hline Mesembrinella 732 & Microchirus 808 & Mixochthonius 720 & Myolepta 309 \\
\hline Mesites 110 & Microcosmus 619, 801 & Mizaphis 190 & Myopsocus 589 \\
\hline Mesoacidalia 194, 316, 358, 549, & Microctonus 489 & Mnemonica 80 & Myospila 365 \\
\hline $676,694,712$ & Microcymatura 290 & Mogoplistes 706 & Myothorax 551 \\
\hline Mesoacidaria 315 & Microdynerus 393, 584, 683 & Molgula 801 & Myothyria 204 \\
\hline Mesocarabus 75 & Microgaster 41, 656 & Molopidius 82 & Myriapora 807 \\
\hline Mesocerus 501 & Microgastrura 461 & Molops 82 & Myrmacyba 733, 772 \\
\hline Mesochelidura 344 & Microhodotermes 506 & Moma 524,560 & Myrmarachne 444 \\
\hline Mesochorus 274, 347 & Microhoria 697 & Mompha 370,562 & Myrmecaelurus 22, 396, 618, 686 \\
\hline Mesocoelopus 435 & Microlestes 38, 92, 96, 219, 631, & Monacha $757,768,782$ & Myrmecia 457, 506 \\
\hline Mesocrambus 382, 548 & 700 & Monacis 634 & Myrmecina 634,72 \\
\hline Mesogona 95, 429, 560 & Microlomalus 723 & Monanthia 208 & Myrmecocystus 457,506 \\
\hline Mesolestes 631 & Microloxia 61, 170, 228, 548, & Monanus 119 & Myrmecozela 332 \\
\hline Mesoleuca 170 & 549 & Moniezia 419 & Myrmelachista 634 \\
\hline Mesoligia 560 & Micromalthus 600 & Monima 95, 549, 560 & Myrmeleon 22, 177, 293, 396, \\
\hline Mesophylax 494 & Micromalus 723 & Monochamus 163, 290, 746 & 618,686 \\
\hline Mesopsocus 589 & Micromus 686, 736 & Monochirus 808 & Myrmeleotettix 727, 737 \\
\hline Mesosa 290 & Micronecta 682 & Monochroa 405 & Myrmetes 723 \\
\hline Mesostena 100 & Microplax 208 & Monogyropus 502 & Myrmica $33,455,457,570,767$ \\
\hline Mesostenus 319, 336, 372 & Microporella 807 & Monohammus 290 & 802 \\
\hline Mesotrichapion 733 & Microppia 720 & Monohelea 632, 664 & Myrmicocrypta 634 \\
\hline Mesotype 170 & Micropsectra 532, 587 & Monolepta 260, 456 & Myrmidius 501 \\
\hline Mesovelia 682 & Micropsocus 589 & Monomorium 600, 634, 725 & Myrmilla 458 \\
\hline Messor 18, 33, 39, 120, 373 & Micropteryx 357,562 & 767,802 & Myrmosa $127,365,458,722$ \\
\hline $457,600,625,6$ & Micropterna 494 & Monopelopia 532 & Myrmosina 722 \\
\hline $658,692,695$ & Micropteryx 80 & Monophlebus 666 & Myrmosula 722 \\
\hline $765,767,802$ & Microptinus 748 & Monopis 332, 371 & Myrmus 501 \\
\hline
\end{tabular}




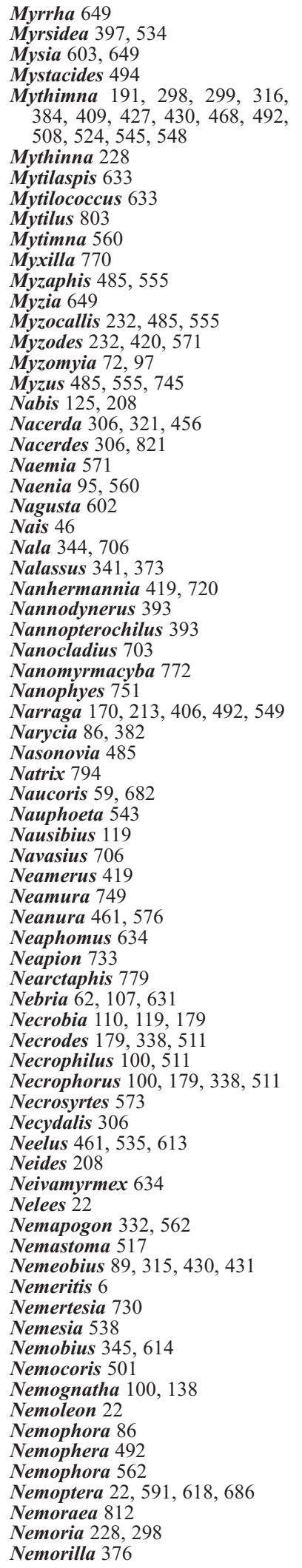

Nemoscolus 538

Nemotois 86, 357

Nemotragus 290

Nemoura 282, 707

Nemurella 707

Nenethes 302, 667

Nenus 736

Neoaplectana 489

Neoascia 394

Neobisium 82

Neobrachychthonius 720

Neochalcis 799

Neochrysopus 163

Neoclosterus 290

Neocnemis 110

Neocyba 733, 772

Neofaculta 405

Neoforelius 634

Neofriseria 405

Neogastrura 461

Neoharpyia 643

Neohipparchia 229, 315, 386 409, 545, 549

Neoleria 756

Neoliochthonius 760

Neoliodes 720

Neolocoptiris 602

Neomysia 582, 649, 680

Neomyzaphis 232

Neonitocris 290

Neootruma 634

Neophron 573

Neopsocus 589

Neopurcelia 517

Neopycnodonte 730

Neoribates 419

Neosapaphis 485

Neoscona 688

Neosphaleroptera 198, 405

Neotipus 656

Neotrichoppia 593

Neottiglosa 208

Neoxystoma 733

Neozavrelia 532

Nepa 208, 506, 682

Nepenthes 46

Nepenthophilus 480

Nepha 140, 631

Nephasitus 22

Nephila 463, 486, 503, 538

Nephodes 114

Nephodinus 341

Nephopteryx 298, 316, 382, 549

Nephotettix 558

Nephus 603, 680

Nepticula 332

Nerium 208

Nesacinopus 110

Nesiominus 588

Vesoporogaster 620

Nesotes 291, 341

Netelia 667

Netocia 681

Netta 588

Nettodarus 808

Neumania 687

Neuria 468

Neurigona 347

Neurocentropus 494

Neuroleon 22, 396, 590, 618, 686

Neuropterus 653

Neuroterus 60, 668, 753

Neurothaumasia 332

Newsteadia 666

Nezara 125, 208

Nicobium 435, 484
Nicodrilus 781

Nicrophorus 338

Niditinea 332, 386

Nidularia 251, 666

Niesthrea 501

Nilaparvata 558

Nilotaspis 633

Nimboa 686, 806

Nimus 705

Nipaecoccus 251, 666

Niphocepheus 720

Nipponorthezia 666

Nirmus 515, 534, 588, 705

Nisoniades 37

Nitela 651

Nitidula 512, 551, 570

Nitocris 163, 290

Noctua 298, 384, 386, 397, 420, $427,508,524,545,546,548,560$

Nodaria 98, 545, 560

Nola 95, 560

Nomada 129, 289, 365, 617

Nomadacris 443, 506

Nomamyrmex 634

Nomia 289, 654

Nomioides 289, 758, 780

Nomophila 155, 228, 229, 298, $382,548,549$

Nonagria 98, 560

Nonyma 290

Nops 444

Nordmannia 19, 660

Norelona 768

Normalis 298

Noserocera 290

Notaphus 96, 631

Notapsis 488

Notaspis 720

Noterus 122, 161, 600, 698

Nothidris 634

Nothochrysa 22

Nothomyrmosa 722

Nothophysis 290

Nothris 405

Nothrus 419, 720

Notidobia 494

Notidocharis 724

Notiophilus 62, 96

Notocelia 405

Notochilus 208

Notocyrtus 733

Notodecta 506

Notodonta 61, 90, 466, 563, 643

Notodromas 537

Notoedres 445

Notoglossa 365

Notonecta 59, 131, 208, 264, 682

Nototrachys 336

Notoxus 219, 456

Notozus 572

Novius 78, 140, 498, 680

Novotinea 332

Nuctenea 688

Nuculaspis 633

Nudaria 95, 560, 580

Nudaurelia 506

Numenius 588

Numida 588

Nupserha 290

Nusalala 736

Nustera 630

Nychiodes 170, 545

Nyctegretis 382

Nycteola 98, 524, 549

Nycteridocoptes 445

Nyctia 365
Nyctopais 290

Nymphalis 76, 104, 190, 195, 236 , $315,316,386,409,410,431$, 492, 526, 548, 549, 660, 676, 694, 712

Nymphopsocus 589

Nymphula 45, 382, 481

Nysius 125, 208

Nysson 129, 608

Nytha 12,16

Oar 170, 300

Oberea 185, 290, 391, 569

Obereopsis 290

Obraztsovia 388

Ochetomyrmex 634

Ochetopus 602

Ochetostethus 208

Ochina 324

Ochlodes 101, 315, 410, 549

Ochodaeus 600

Ochromolopis 357

Ochropleura 95, 189, 190, 298,

$316,524,545,548,549,560$

Ochrosis 260, 277

Ochrostigma 90, 492, 643

Ochsenheimeria 332

Ochterus 208, 682

Ochthebius 456, 529, 648, 699

Ocladius 697

Ocnera 100

Ocneria 98, 213, 560

Ocnerodes 706

Ocnerostoma 370

Ocnogymna 333, 426, 431, 545

Ocnogyna 41, 68, 80, 95, 136, 274, 302, 492, 509, 543, 545, $548,549,560,580$

Octopus 790

Octostruma 634

Ocularia 290

Ocydromus 62, 96, 325, 391, 631

Ocypus 681

Ocys 38, 631

Odezia 170, 548, 549

Odinophora 129

Odonestis 101, 429, 545, 549

Odontaeus 237

Odontella 461, 740

Odontocepheus 720

Odontocerum 494

Odontodynerus 393

Odontognophos 170

Odontomachus 634

Odontonyx 62, 96, 140

Odontopera 468

Odontoscelis 125, 208

Odontosia 90, 545, 549, 643

Odontotarsus 125

Odontotermes 506

Odontothrips 615

Odontura 716

Odynerus 33, 81, 183, 296, 317, $393,450,499,513,584,683,713$

Oeax 290

Oebia 298

Oecanthus 49, 345, 543, 614

Oeceoptoma 179, 511

Oecetis 494

Oecia 405

Oecophora 468, 562

Oecophylla 457, 506

Oecothea 756

Oedaleus 345, 716

Oedalus 706

Oedemera 306, 456, 818

Oedemerina 306 
Oedibria 61

Oedibrya 98,546

Oedionychus 68

Oedipoda 226, 345, 506, 614, 672, 706, 716, 727, 737

Oedipora 706

Oegoconia 382

Oenas 138, 219

Oenopia 649, 680

Oestophora 761, 768

Oestophorella 768

Ogmoderus 484

Ogulnius 444

Ogygia 524

Oiceoptoma 338

Oidaematophorus 228, 382

Oinophila 332

Olene 213, 560

Olethreutes 198, 229, 405, 562

Oligia 427, 545, 546, 560

Oligochroa 382

Oligomerus 484

Oligomyrmex 634

Oligonichus 346

Oligoplectrum 494

Oligotrophus 806

Olios 538

Olisthopus 92

Olistophus 38

Olnyx 753

Olocrates 230

Omacantha 506

Omalogyra 750

Omalosecosa 807

Omalus 129, 458, 572, 713

Omaseus 391

Omia 95, 560

Ommatocepheus 708, 720

Omocestus 345, 614, 706, 716, 727,737

Omoperyphus 631

Omophlus 219

Omophron 38, 631

Omorgus 600

Omosita 512, 570

Omphalapion 733

Omphalophana 61, 95, 560

Omphaloscelis 61, 98, 190, 545 $548,549,560$

Oncocephalus 125, 208, 602

Oncocnemis 546, 560

Oncomera 818

Oncopodura 461

Onebala 405

Onesia 179, 365

Onipchora 61

Oniscus 674

Oniticellus 28, 186

Onitis 237, 506

Onthophagus 28, 186, 220, 237,

$477,663,816$

Onthophilus 723, 817

Onuxicera 204

Onychapion 733, 772

Onychiurus 461, 535, 576, 613

Onychocera 204

Onychogomphus 647

Onycholips 110

Onychora 170, 545

Oochrotus 120, 341, 373

Oodes 220

Oophorus 110, 391

Opaca 416

Opatropis 110, 140, 291

Opatrum 96, 120, 172, 280, 341 697
Operophtera 61, 170

Operophthera 45

Ophion 127, 190, 274, 302, 336, 667

Ophionea 38, 322

Ophiusa 61, 98, 191, 430, 548, 549,560

Ophonus 38, 62, 75, 92, 96, 631. 700

Ophyra 179, 365

Opilio 487

Opilo 183, 477

Opinus 602

Opisthograptis 170, 229

Opito 88

Opius 577

Oplistopus 602

Oplomerus 393

Opomala 614

Oporinia 170, 211, 549

Opostega 332

Oppia 419, 488, 593, 720

Oppiella 720

Opsibotys 382

Opsilia 569

Optsicoetus 602

Orbellia 756

Orchesella 461

Orenaia 382

Oreocarabus 631

Oreopsyche 80, 86, 89, 136, 382 , 406, 430, 562

Orestia 260

Orfilaia 600

Orgyia 98, 213, 406, 508, 545, 549,560

Oria 61, 98, 549, 560

Oribata 419, 720, 741

Oribatella 419, 720

Oribates 720

Oribatula 419, 708, 720

Oribella 720

Oribotritia 419, 720

Orictes 35

Orinocarabus 62, 261

Oriolus 397

Ormyrus 653

Ornativalva 405

Ornithobius 588

Ornithodoros 445

Ornithonissus 445

Ornithopeplechthos 515

Orophia 562

Orphne 170

Orrhodia 560

Orsodacne 238

Orthetrum 83, 264, 396, 574, 647

Orthezia 163, 666

Orthezinella 666

Orthocladius 32, 565, 665, 785

Ortholitha 154, 170, 211, 228 , $229,429,549$

Orthomus 631, 700

Orthoneura 394

Orthopelma 636

Orthoperus 630

Orthophilus 723

Orthosia 95, 427, 546, 560

Orthotaenia 198, 405, 562

Orthotomicus 456

Orthotrichia 494

Ortygis 113

Oryctes 127, 163, 214, 220, 321 ,

$361,506,600,630$

Oryssus 175, 303

Oryxolaemus 733
Oryzaephilus 100, 119, 308

Oscinella 519

Osellaeus 733

Osmia 58, 138, 200, 201, 268, 289.

$320,439,499,637,713,758,780$

Osmoderma 88, 214

Ostoma 570

Ostrinia 228, 382, 548, 549, 557. 558

Osydromus 96

Otala 757,768

Otholitha 228

Otidoecus 705

Otilipeurus 705

Otiocephala 266

Otiorhynchus 681, 697

Otiorrhynchus 92, 110, 391

Otis 705

Otodectes 445

Otus 397

Oudablis 666

Oulimnius 648

Ourapteryx 170, 545

Ovaticoccus 666

Ovatomyzus 745

Ovatus 485, 555

Oxicesta 95, 524, 560

Oxipleurites 346

Oxiptilus 548

Oxus 687

Oxya 506

Oxybeloides 365

Oxybelomorpha 365

Oxybelus $127,129,250,365,531$, $608,621,651,685,713,722$

Oxybia 382

Oxycara 110

Oxycaremus 125

Oxycarenus 208

Oxycarops 110

Oxycaula 290

Oxycesta 492

Oxychilus 757,782

Oxyepoecus 634

Oxyethira 494

Oxygastra 264

Oxyhammus 290

Oxylaemus 484

Oxylamia 290

Oxylipeurus 473

Oxyomus 600

Oxyopomyrmex 725,802

Oxypoda 27

Oxypteron 198, 405

Oxyptilus 229, 382

Oxystoma 733, 75

Oxystrongylus 570

Oxytelus 630

Oxythrips 609, 615

Oxythyrea 100, 139, 186, 214, 681

Oysaphis 670

Pachetra 560

Pachisema 193, 337

Pachnephorus 456

Pachnoda 100

Pachybrachis 260

Pachybrachys 219

Pachychila 36, 47, 92, 261, 341

Pachychyrus 818

Pachycnemia 170, 549

Pachycondyla 634

Pachydema 214

Pachydissus 290

Pachygastria 386, 492, 545

Pachyleuctra 707

Pachylister 456, 723

Pachylomma 175, 263

Pachylopus 110, 723

Pachypasa 101, 316, 545, 549

Pachystola 163, 290

Pachythelia 86, 382, 468, 549

Pachytychius 391, 751

Pactolinus 723, 817

Paederus 110, 436, 470

Pagellus 808

Pagurus 710

Paidia 61, 95, 229, 689, 560, 580

Paivea 110

Pakesia 602

Palaeacarus 445

Palaemon 619

Palaeococcus 666

Palaeolecanium 650

Palarus 129, 685

Paleira 110, 214

Palemochartus 193

Palethrocoris 501

Palinurus 27

Palluperina 61, 98, 549, 560

Palmitia 382, 549

Palmodes 684, 685

Palomena 208

Palorus 47, 110, 308

Palpares 22, 618

Palpita 228, 298, 382, 545, 549

Palpomyia 632, 664

Paltodora 370

Paltothyreus 457

Pamera 208

Pammene 198, 405

Pamphila 316

Pamphilius 476

Panagaeus 96, 325

Panaxia 101, 429, 453

Pancalia 370

Panchrysia 560

Pandalina 619

Pandemis 198, 405

Pandesma 298, 427, 560

Pandoriana 104, 134, 315, 316, 358, 549, 660, 676, 694, 712

Panemeria 98, 492, 560

Pangonia 163, 586

Pangonius 554, 586

Panimerus 232

Paniscus 372, 667

Panolis 61, 95, 302, 400, 549, 560

Panonychus 445

Panorpes 458, 713

Panstegia 382, 545

Pantelozetes 720

Panurginus 289

Panurgus 138, 289, 458, 637, 758

Papilio 8, 12, 37, 41, 61, 76, 93,

104, 113, 190, 194, 229, 236,

$315,316,385,406,410,429$,

$430,433,453,468,526,544$,

$545,548,549,629,660,759$ 


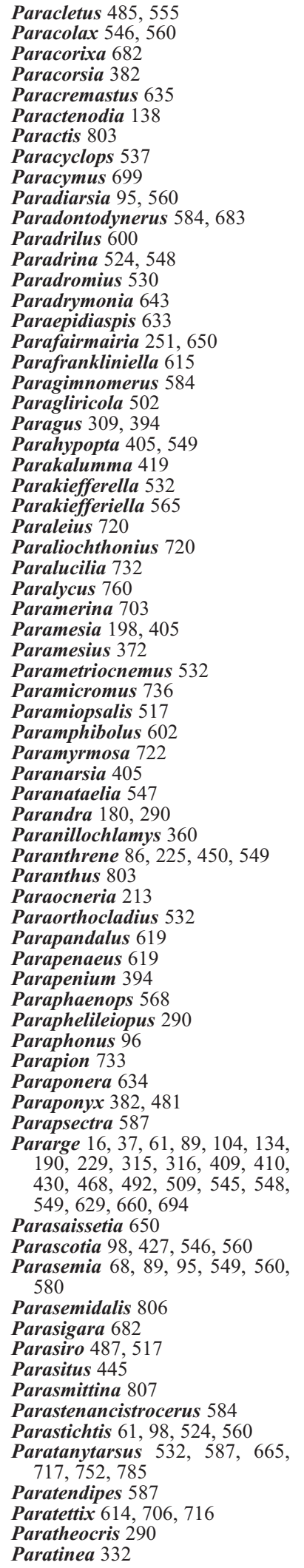

Paratrechina 634

Paratrichocladius 532, 565

Paratullbergia 743

Paratyphlus 63

Paravespula 296

Parawammerdamia 370

Parazetes 419

Pardia 405

Pareulype 170

Parexochaeneus 249

Parhypochtonius 720

Parlatorea 633

Parlatoria 163, 251, 364, 396 , 582,633

Parmena 290, 697

Parnara 101, 157, 315, 409, 549

Parnassius 41, 45, 51, 74, 76, 80, 89

$104,113,136,169,189,229,252$

$315,316,385,386,406,409,433$

$453,454,492,507,526,549$

Parnopes 127

Paroberea 290

Parodontodynerus 393

Parodynerus 317

Paroeax 290

Paroeme 290

Paromalus 723, 817

Paronychora 189

Parophonus 700

Paroplites 506

Parornix 357, 562

Parthenolecanium 650

Parurus 303

Parus 397

Paryphoclonius 290

Pasiphaea 619

Pasira 602

Pasites 289, 320, 617

Passaloecus 250, 439, 652, 684 685

Passalozetes 488, 720

Passalus 506

Patanga 506

Pavo 588

Peachia 803

Pechipogo 98, 524

Pectinophora 405, 468, 548

Pectinopygus 397

Pediasia 228, 316, 382, 430, 491 , 492, 548

Pediaspis 753

Pediculines 705

Pediculoides 6, 445, 518

Pediculus 44, 57, 502, 515, 534 573, 588

Pegomyia 330, 420, 514

Peirates 176

Pelatea 198, 405

Pelecocera 394

Pelochares 600

Pelopeos 33

Pelopoeus 129

Pelops 720

Peloptulus 720

Pelor 62

Peloribates 720

Pelosia 95, 315, 546, 560, 580

Peltis 570

Peltodytes 161, 600, 698

Peltonychia 517

Peltotrupes 69

Pempelia 228, 229, 382

Pemphigus 215, 232, 485, 555

Pemphredon 129, 232, 250, 439,

$572,652,684,685,713$
Penaeus 619

Penestoglossa 86, 382

Penetretus 631

Pentalonia 232, 485

Pentaneura 313

Pentapedilum 532, 587, 717

Pentaphyllus 47

Pentapora 807

Pentatemnus 110, 291

Pentatoma 208

Penthaleus 346

Pentodon 92, 100, 139, 186, 214, Phaneroptera 345, 614, 706 600

Pentomeura 565

Pepsis 450

Peranus 723

Perapion 733, 751, 772

Perarge 358

Percnoptilota 170

Perconia 170

Percosia 96

Percus 92

Perdix 588

Perforatella 768

Pergalumna 419, 720

Pergesa 386

Peribalus 208

Pericerya 722

Periclista 347

Periclistus 636, 668

Peridea 563, 643

Peridroma 95, 228, 316, 384 , $468,548,549,560$

Perileptus 38, 110, 631

Perilissus 129

Perilitus 193, 571

Perinephela 228, 382

Periphanes 468, 560

Periphyllus 555

Peripion 733

Periplaneta 345, 506

Peripsocus 589

Perissomyrmex 634

Peristedion 808

Perithous 372

Peritrechus 208

Perittia 357

Perizoma 170, 189, 190, 467

Perla 282, 707

Perlamantis 706

Perlamantispa 591, 618, 686

Perlodes 282, 707

Perlohmannia 419

Pernoctilota 298

Pernoptilota 548

Perophora 801

Perriniella 27, 360

Perspicuoppia 720

Petaloptila 345

Petilampa 98, 191, 560

Petrobius 542

Petrognatha 163, 290

Petrova 198, 405

Pexapion 733

Pexicopia 405

Pezomachus 175

Pezotettix 345, 706, 716

Pezothrips 615

Phaedon 238, 305, 701, 723

Phaenicia 732

Phaenocarpa 514

Phaenodiscus 251, 364

Phaenolobus 372

Phaenops 183

Phalacromyrmex 634

Phalacropterix 86, 382
Phalacrotophora 571

Phalacrus 232

Phalaena 170, 213, 298, 371, 427, 429, 467

Phalangium 487, 517

Phalera 61, 90, 302, 548, 563, 643

Phaleria 47, 96, 100, 110, 120, 291, 341, 413

Phallusia 619, 801

Phalonidia 198, 405, 562

Phaloropus 588

Phaneta 545

Phanetoprepa 405

Phantasmatophanes 602

Phaonia 489

Pharetra 524

Pharmacis 357

Pharoscymnus 680

Phaselia 170

Phasgonura 404

Phasiane 492

Phasianus 588

Phauloppia 708, 720

Phauloppiella 720

Pheidole 18, 39, 373, 600, 634, $692,715,719,725$

Pheidolle 802

Pheidologeton 39

Phelister 723

Phenacoccus 251, 649, 666

henopelops 720

Pheosia 90, 643

Pheropsophus 100

Phiarus 289, 320

Phigalia 170, 467

Philaeus 444, 463, 503

Philanthus 250, 608, 621, 685, 713

Philea 158, 560, 580

Philedone 198, 405

Philedonides 198, 405

Philematium 290

Philereme 170

Philhammus 240

Philhydrus 529

Philipomyia 586

Philippia 650

Philochthus 96

Philochtus 631

Philodesma 545

Philomachus 588

Philonthus 110, 436

Philontus 140

Philopedon 391

Philophylla 577

Philopomyia 554

Philopotamus 494

Philopterus 397, 502, 534, 588

Philorhizus 62, 220

Philosamaia 283

Philosamia 101, 157, 283, 549

Philotarsus 589

Philotes 660

Philudoria 101

Phlebiomus 736

Phlebotomus 5, 14, 17, 25, 124, 702

Phloemyzus 485

Phloeobius 163

Phloeomyzus 555

Phloeophilus 600

Phloeothrips 216

Phlogophora 192, 384, 420, 446, $545,546,548,560$

Phoenicopterus 113

Phoeocritus 723

Phoeogenes 263 
Pholcus 444, 503, 538

Pholidoptera 614

Phonapate 239

Phora 128, 179

Phormia 179

Phorocera 492

Phorodon 485, 555

Phoroncidia 444

Phosphorus 290

Phosphuga 338, 511

Photedes 191, 386, 560

Phothydraena 648

Photodes 546

Photuris 544

Phoxinus 744

Phradis 635

Phragmataecia 86, 405

Phragmatecia 86, 549

Phragmatiphila 98

Phragmatobia 61, 95, 158, 191 $429,548,560,580$

Phrealcia 357

Phremia 506

Phrissoma 290

Phrissotrichium 733

Phrissotrichum 733, 751, 772

Phrosinella 365

Phrosyne 290

Phrygaena 589

Phryganea 494

Phrygaenopsis 560

Phryganopsis 298, 580

Phryneta 163, 290

Phrynetoides 290

Phrynetopsis 290

Phtheochroa 198, 405

Phthiracarus 419, 720

Phthirius 44, 57

Phthorimaea 69, 405, 468

Phthrimaea 548

Phycis 298, 808

Phycita 224, 298, 316, 382, 406

Phycitodes 545

Phygadeuon 514

Phyla 325, 391

Phylaeus 463

Phylan 3, 47, 88, 230, 341, 373, 412,697

Phylhydrus 456

Phyllaphis 232, 485

Phyllarthrius 290

Phyllecoptruta 445

Phyllobius 110, 751

Phyllocnistis 357, 387

Phyllocoptes 346

Phyllocoptruta 346

Phyllodecta 260

Phyllodromica 345, 621, 706

Phyllognathus 140, 186, 214, 600

Phyllognatus 214

Phyllomorpha 59, 208, 501

Phyllonorycter 562

Phyllopertha 186, 214

Phyllophila 98, 228, 549, 560

Phyllophora 555

Phyllotreta 219, 260, 304

Phylloxera 232, 485

Phyllozetes 720, 760

Phylognathus 100

Phymatechus 290

Phymatodes 630

Phyrrhocorus 125

Physa 264

Physiphora 500

Physocephala 500

Physocyclus 444, 503
Physokermes 650

Physoskermes 251

Physostomum 515

Physothrips 615

Phytodecta 260, 277

Phytoecia 110, 290, 391, 569, 746

Phytometra 61, 98, 102, 228 , 549, 560

Phytomiza 337

Phytomyza 193, 314, 365, 387

Phytonomus 391, 412

Phytoptus 346

Phytoseius 445

Phytosus 110

Piarus 110

Pica 397

Picromerus 208

Picus 397

Pieris 12, 33, 37, 41, 61, 73, 89

$104,134,135,140,195,229$

$236,315,316,358,367,386$,

$409,410,430,433,455,468$

$492,509,526,545,546,548$

$549,610,629,659,660,694$.

712,759

Piesma 472

Piezodorus 125, 163

Piezotrachelus 733

Pilogalumna 419, 720

Pimelia 47, 92, 100, 106, 110, 119 140, 291, 341, 412, 506, 697

Pimpla 129, 263, 336, 372, 376, 656

Pinacosterna 290

Pinacosternodes 290

Pinna 619

Pinnaspis 163, 633

Pinnotheres 619

Pinnothetes 710

Piona 687

Pionopsis 687

Pionosomus 208

Pionosyllis 742

Piophila 179, 477

Piotes 110

Pipiza 232, 394

Pipizella 215

Piraphis 232

Pirapion 733, 751

Pirata 503

Pirates 125, 176, 602

Pirnodus 419, 720

Pisa 710

Pisanura 486

Pisidia 710

Pisidium 786

Pison 129, 651

Pissodes 183, 224, 321, 75

Pitiophagus 373

Pitymys 420, 446

Pityogenes 141, 723

Pityophagus 512

Placodoma 382

Plagiodera 304

Plagioecia 807

Plagiognatus 232

Plagiolepis 634, 656, 715, 719 725,802

Plagiomus 290

Plagionotus 88

Plagiotrochus 653, 668, 669, 753

Plagodis 170

Planasida 47

Planchonia 251, 650

Planeustomus 110

Planococcus 235, 251, 666
Planodema 290

Plarylomanus 723

Plateremaeus 419

Plateumaris 257

Plathyrrhinus 88

Platichthys 744

Platiplax 208

Platyarthrus 674

Platybunus 517

Platycephala 110

Platycerus 214, 390

Platychirus 394

Platycis 600

Platycleis 345, 612, 614, 621, Poecilochthonius 720

$$
\text { 706, } 716
$$

Platycnemis 318, 647

Platydactylus 264

Platydema 88

Platyderus 220, 325

Platyedra 405

Platygenia 506

Platylabus 129

Platylomalus 723

Platynaspis 232, 582, 680

Platynothrus 419, 720

Platynus 38, 359

Platyperigea 524

Platyplax 125

Platypleura 506

Platypsyllus 600

Platyptilia 382, 545

Platypus 180, 321, 600

Platyrrhinus 321

Platysma 96, 631, 700

Platysoma 88, 701, 723, 817

Platysomum 817

Platystethus 100

Platytarus 96, 171, 322, 412

Platytes 229, 382

Platythyrea 634

Plea 682

Polydrosus 110

$135,154,156,189,191,195, \quad$ Polydrusus 697, 75

209, 228, 229, 315, 316, 358, Polyergus 457, 723

$372,386,406,408,409,410$, Polygonapion 733

430, 454, 455, 468, 492, 507, Polygonia 16, 37, 104, 195, 229, $508,545,546,549,712$

Plebicula 430, 503, 507, 545, $546,629,660$

Plectiscus 263

Plectogaster 290

Plectrocnemia 494

Plegaderus 723, 817

Plegadilymantikos 515

Plegederus 817

Plemyria 170, 467

Plentuisa 768

Pleotrichophorus 485, 555, 745

Pleraplysilla 800

Plesionika 619, 710

Plesioprinus 723

Pleurodirus 751

Pleurophorus 92, 96, 100, 139, 186,600

Pleurota 229, 370, 545

Pleuroxus 537

Plocadenus 290

Plodia 6, 382, 548

Ploecaria 602

Ploiaria 125, 602

Ploiariodes 602

Ploiariola 602

Ploiariopsis 602

Plusia 8, 98, 169, 191, 211, 229,

$316,317,384,420,427,429$,

$506,509,548,549,560$

Plutella 229, 357, 549, 562

Pneumonyssus 445

Podagrica 260, 277, 304, 456

Podalonia 621, 684, 685

Podarcis 721

sma 614

Poecilonota 88

Poecilus 38, 62, 96, 100, 631, 700

Pogonistes 322, 412

Pogonoidius 96

Pogonomyrmex 543, 634, 658

Pogonus 38, 96, 100, 110, 322, 412

erus 290

Polietes 179

Polimixis 427, 492

Polister 506

Pollenia 365

Pollinia 251, 650

Polybius 710

Polycarpa 801

Polycelis 744

Polycentropus 494

Polychrysia 560

Polychrosis 9

Polycitor 801

$236,315,316,409,410,453$,

$526,548,549,676,694$

Polygoniac 61

Polymitoleiopus 290

Polymixis 524, 545, 548, 560

Polyochodes 382

Polyommatus 19, 209, 229, 509, 548, 549, 629, 656, 660, 712

Polypedilum 532, 587, 717, 752

Polyphaenis 98, 524, 545, 546, 560

Polyphilla 662

Polyphyla 186

Polyphylla 139, 214, 420

Polyploca 90, 263, 405

Polypogon 546, 560

Polyrrahphis 290

Polysphincta 336

Polysyncraton 801

Polytoxus 602

Pomatias 613

Pompilus 608

Ponentina 768

Ponera 457, 600, 725

Pontia 12, 61, 89, 93, 104, 135.

$190,194,229,236,315,316$

$358,410,424,430,455,526$,

$545,548,549,629,659,660$, 694, 712 
Pontophilus 619, 710

Popilia 780

Popillia 44

Porcellio 462, 674, 675

Porizon 635

Porobelba 720

Porotachys 631

Porpe 370

Porphyrinia 61, 98, 191, 298,

$316,386,427,430,468,524$.

$548,549,560$

Porphyrio 113

Portethria 548

Porthesia 347

Porthetria 333, 400, 406, 508

$511,543,545,549,558,560$

Portugala 768

Portumnus 710

Porzana 588

Postsalebria 382

Potamocypris 537

Potamonectes 122, 698

Potamophylax 494

Potamopyrgus 744

Potasida 47

Potosia 214

Powelia 37, 61, 89

Powellinia 560

Praemachilis 575

Praemachiloides 575

Praetinea 332

Pranticola 397

Praon 232

Prasocuris 304

Prays 357, 375, 464, 548

Prenantia 807

Prenolepis 634, 656

Pria 63, 512

Priaspis 193

Princidion 96

Princidium 631

Prionopelta 634

Prionoplus 506

Prionopoda 129, 274, 336

Prionoteca 100

Prionotylus 501

Prionus 88, 290, 746

Prionychus 88,32

Prionyx 684, 685

Pristomerus 129, 336

Pristonychus 82, 360, 631

Pristophorodes 382,

Proagosternus 506

Probaticus 341, 391

Probezzia 664

Probles 635

Probolomyrmex 634

Procapperia 382

Proceratium 634

Processa 619, 710

Procinetus 129, 372

Procladius 565, 587, 752, 785

Procris 61, 111, 405, 406, 409, 492, 549

Procrustes 261

Procryptocerus 634

Proctenius 681

Proctocera 290

Proctostephanus 461

Procus 98, 211, 549

Procustes 96

Prodenia 98, 143, 299, 398,560, 623

Prodiamesa 532

Prodomitia 290

Proecilictis 397
Proformica $715,719,767$

Proisotoma 461, 474, 488, 763

Promalactis 370

Promesomachilis 575

Promethes 319

Pronotestra 95, 560

Propelodytes 787

Propiromorpha 198, 405

Proporcellio 674

Propylaea 219, 582, 649, 680

Prorops 163

Proscus 263

Proserpinus 74, 101, 157

Prosopigastra 651, 684

Prosopis 289, 320, 439, 458, 654

Prosopocera 290

Prosopothrips 609

Prosphorus 290

Prostemma 208

Prosternoptinus 748

Prostoma 813

Prostomis 119, 308, 600

Protalaridris 634

Protaphis 485

Protapion 733, 75

Protargionia 633

Prothymnia 113, 560

Protolachnus 485

Protomutilla 722

Protonemura 707

Protoperyphus 110

Protophormia 179

Protopirapion 733, 751

Protopulvinaria 251, 650

Protorhoe 170

Protoribates 720

Protoschinia 545, 560

Prototrichapion 733

Proutia 382

Psacasta 208

Psacothea 506

Psalis 592

Psammaecius 608

Psammathocrita 405

Psammobius 28, 92, 96, 100

139, 186, 220

Psammochares 127

Psammodius 600

Psammotis 382

Psectrocladius 565, 665, 785

Psectrotanypus 24, 46

Psedargyrotozoa 198

Pselactus 391

Pselnophorus 382

Psen 129, 392, 439, 652

Psenulus 250, 392, 499, 652

Pseudachorudina 461, 728

Pseudachorutes 461, 535

Pseudagapetus 796

Pseudallosterna 630

Pseudanchomenus 110

Pseudanthidium 637

Pseudanurophorus 461

Pseudaphycus 364

Pseudapion 733

Pseudaplemonus 733

Pseudargyrotoza 405

Pseudatemelia 562

Pseudechinosoma 110

Pseudenargia 548

Pseudepierus 723

Pseudepipona 393, 584

Pseuderos 290

Pseudeucosma 149, 198

Pseudhammus 290

Pseudoaonidia 251
Pseudoapterogyna 679

Pseudoatta 634

Pseudobargylia 602

Pseudoblepisansis 290

Pseudocamptodes 570

Pseudocatapion 733

Pseudochariesthes 290

Pseudochazara 315

Pseudochelidura 242, 592

Pseudochermes 666

Pseudochrysis 81

Pseudocleonus 681

Pseudoclerops 183

Pseudococcus 78, 163, 251, 666

Pseudococcyx 198, 405

Pseudoconapion 733

Pseudoconis 806

Pseudocosma 405, 545

Pseudocypus 681

Pseudodistoma 801

Pseudoenargia 546, 560

Pseudogyps 573

Pseudohadena 98, 427, 492, 546,

Pseudoips 95, 508, 560

Pseudolucanus 214

Pseudomasoreus 171

Pseudomenopon 588

Pseudometapion 733

Pseudomyas 110

Pseudomyrmex 634

Pseudomyrmosa 722

Pseudonupserha 290

Pseudopanthera 170, 549

Pseudoparlatoria 633

Pseudoperapion 733

Pseudophilotes 655, 712

Pseudophlaeus 208

Pseudophloeus 501

Pseudophotopsis 722

Pseudopiezotrachelus 733

Pseudopipona 683

Pseudopirapion 733

Pseudoppia 419, 720

Pseudoprotapion 733

Pseudoreduvius 602

Pseudorhinus 391, 681

Pseudorthocladius 785

Pseudoseriscius 110, 120, 341

Pseudosinella 461, 474, 535, 613, 783

Pseudosmittia 532, 565

Pseudostenapion 733

Pseudoswammerdamia 370

Pseudotachea 768

Pseudotalpophila 110

Pseudotectoribates 720

Pseudotelphusa 405

Pseudotergumia 104, 228, 236,

$315,545,548,549,660,694$

Pseudoterpna 170, 228, 298, 549

Pseudotomoides 405

Pseudotrichapion 733

Pseudotritia 419, 720

Pseudovespula 296

Pseudoxenos 621

Pseudoyersinia 706, 814

Pseudozarba 560

Psila 193, 784

Psilachnopus 240

Psilochalcis 799

Psiloderes 363

Psilomonodes 98, 560

Psiloptera 100

Psilopteryx 494

Psilosage 336, 347

Psilota 797

Psilotrix 456

Psilunio 786

Psithyrus 289

Psoa 484

Psococerastis 589

Psocus 589

Psodocerus 506

Psodos 170, 195

Psoricoptera 405

Psorophora 754

Psoropteres 162

Psoroptes 445

Psorosa 298, 382

Psotilnus 501

Psyche 86, 382

Psychidea 86

Psychomyia 494

Psylla 232, 499, 506

Psylliodes 219, 260, 456

Psyllioides 304

Psyllipsocus 589

syllopsis 499

Psylophrys 251

Ptenotrix 461

Pterapherapteryx 467

Pterapion 733, 772

Pterocallis 232, 485

Pterocheilus 129, 317, 584

Pterochthonius 419

Pterocles 113

Pterocomma 485, 555

Pterocroce 686

Pterodela 589

Pterogon 8, 492

Pterolamus 376

Pterolasia 100

Pterolepis 345

Pterolonche 370

Pterolophia 290

Pteromalus 33, 367, 636

Pterophagus 515

Pterophorus 382, 492

Pteroptrix 251

Pteroschichus 38

Pterosoma 61

Pterostichus 62, 107, 489, 568

Pterostoma 90, 545, 563, 643

Pterothrix 228, 382, 545

Pterotopteryx 370

Pterotragus 290

Ptilinus 88, 324, 435

Ptilocolepus 494

Ptilodon 643

Ptilodontella 643

Ptilophora 90, 643

Ptinus 110, 179, 219, 456, 630, 748

Ptocheuusa 405

Ptomaphagus 360

Ptycholaemus 290

Ptycholoma 198, 405

Ptycholomoides 546 


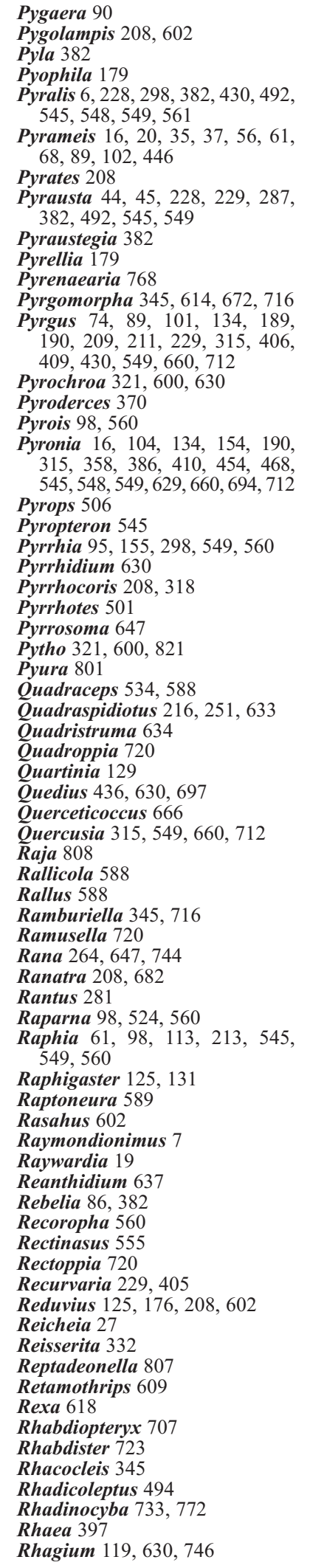

Rhagoletis 578

Rhagonycha 321

Rhamna 435

Rhantus 77, 122, 161

Rhaphidopalpa 260

Rhaphidosoma 602

Rhaphigaster 208

Rhegmatophila 90, 157, 643

Rheotanytarsus 46, 532, 703, 785

Rhina 506

Rhinapion 733

Rhinchophorus 506

Rhinocoris 176, 602

Rhinonyssus 445

Rhinophora 365

Rhinosimus 88

Rhipicephalus 445

Rhizarcha 193, 337

Rhizaspidiotus 633

Rhizedra 98, 560

Rhizobius 219, 498, 582, 680

Rhizococcus 666

Rhizoecus 251, 666

Rhizoglyphus 445

Rhizogramma 560

Rhizopertha 110, 119, 239, 630

Rhizophagus 179

Rhizopulvinaria 650

Rhizotrogus 186, 202, 214, 248

$259,506,527,662,679,681,697$

Rhizotype 61, 98, 549, 560

Rhodites 127, 636

Rhodnius 611, 626

Rhodobium 485

Rhodocleptria 61, 95, 155, 316 548,560

hodometra 61, 170, 228, 229

$298,545,548,549$

Rhodophaea 113, 382

Rhodostrophia 61, 157, 170, 228 ,

$492,545,548,549$

Rhomphaea 444

Rhopalaea 801

Rhopalandrothrips 615

Rhopalapion 733

Rhopalirus 290

Rhopalizarius 290

Rhopalizus 290

Rhopalocera 290

Rhopalosiphoninus 555

Rhopalosiphum 485, 555

Rhopalothrix 634

Rhopalum 608, 65

Rhopalus 208, 501

Rhophites 289

Rhopobota 198, 405

Rhoptria 61, 170, 545

Rhyacia 61, 95, 189, 228, 427 , 524,560

hyacionia 198, 405, 406, 549

Rhyacophila 494, 606

Rhygchium 317, 393

Rhynapion 733

Rhynchites 110

Rhynchium 584, 683

Rhynchobanchus 704

Rhynchopacha 405

Rhynchozoon 807

Rhyncolus 110

Rhyncomyia 262

Rhynocoris 208

Rhypagla 560

Rhyparia 95, 560, 580

Rhyparochromus 208

Rhysipolis 577
Rhysodes 600

Rhysotritia 419, 720, 760

Rhyssemodes 100

Rhyssemus 139, 186, 600

Rhytidocerus 140

Rhytidoderes 92, 140, 412

Rhytidoprinus 723

Rhytirhinus 681

Rhytirrhinus 92

Rhyzopertha 484

Rhyzotrogus 8

Ribasia 302

Ricinus 397, 588

Ripersia 251, 666

Rissa 588

Rivetina 706

Rivula 98, 524, 560

Rodolia 119, 219, 603, 680

Roepkea 779

Roeselia 95

Rogeria 634

Rondania 489

Ronisia 458, 713

Rophalosiphum 571

Rophites 637

Ropica 290

Rosalia 88, 108

Rothschildia 433

Rotruda 382

Rubioia 370

Rubiothrips 609

Rugaspidiotus 633

Rugilus 436

Rumicia 19

Rumina 757, 782

Runchomyia 754

Rungsia 555

Ruperezia 405

Rupicapra 29

Rusadiria 138

Rusekella 749

Rusina 546, 560

Ruspolia 706

Rynchophorus 163

Ryssa 336

Ryssemus 96

Sabethes 754

Sabularius 100

Sacium 630

Saemundssonia 588

Saga 673

Sagaritis 129, 263

Sagittarius 573

Saissetia 136, 163, 208, 251, 504, 518,650

Saitis 463

Salmo 744

Saltusaphis 485

Saperda 110, 290, 321, 569

Saphanidus 290

Saphonecrus 668, 669

Saprinus 100,110, 140, 179, 219 456, 681, 701, 723, 817

Saprobia 570

Sapromyza 365

Sapyga 129, 175

Saragossa 95, 492, 524, 560

Sarcogyps 573

Sarcophaga 179, 365, 477

Sarcoptes 21, 445

Sarcotragus 800

Sarisophora 405

Sarops 193

Sarpeda 391

Sarrothripus 61, 211, 560

Sarrothrypus 95
Sastrapada 602

Satrapada 602

Saturnia 2, 41, 61, 76, 93, 101,

$123,133,191,252,302,316$,

$333,334,371,409,424,426$,

$522,543,545,548,549$

Satyrium 712

Satyrus 12, 16, 30, 61, 104, 113,

154, 190, 196, 229, 236, 315,

316, 358, 386, 409, 492, 522,

$548,549,660,694,712$

Savignyella 807

Scaeva 309, 394, 500

Scanthius 208

Scantius 125

Scaphaphorura 734

Scapheremaeus 708

Scaphidium 88

Scaphura 450

Scapsipedus 443

Scarabaeus 92, 100, 139, 186, 226, $237,267,495,506,681,816$

Scarites 38, 96, 100, 106, 110,

291, 322, 506, 700

Scarodytes 698

Scatophaga 365, 500

Scaurus 47, 78, 92, 100, 120, 341,412

Sceliodes 299

Sceliphron 33, 127, 129, 250, 684,685

Sceloporus 721, 739

Scelosodis 100

Schaefferia 461

Scheloribates 419, 488, 708, 720

Schendyla 620

Scherotheca 781

Schiffermuelleria 468

Schistocerca 69, 203, 207, 443, 479, 506, 558

Schistophila 405

Schizaphis 485, 555

Schizapis 571

Schizobrachiella 807

Schizomavella 807

Schizoneura 555

Schizopelex 494

Schizoporella 807

Schizopyga 336

Schizotargionia 633

Schizotheca 807

Schizotus 600, 818

Schizura 466

Schoenobius 382, 468

Schoettella 461, 535

Schrankia 560

Schreckensteinia 370

Sciapteron 113, 225

Sciobia 716

Sciocoris 208

Sciodrepa 100

Scirpophaga 382

Scirtothrips 558

Sclerocona 382

Scleropatrum 172

Sclerosoma 517

Scobicia 269, 484

Scolia 127

Scoliocentra 756

Scolioplanes 620

Scoliopteryx 98, 560

Scolitantides 19

Scolobates 336

Scolopocranium 501

Scolytus 630, 723

Scoparia 229, 382 
Scopeaus 697

Scopula 61, 155, 170, 228, 229,
298, 548, 549

Scorpaena 808

$446,524,546,548$

Scotochrosta 560

Scotodipnus 27, 75

Scotogramma 61, 95, 157, 549

Scotolemon 517, 539

Scotopteryx 545

Scrizoneura 485

Scrobipalpa 386, 405, 420

Scrobipalpula 405

Scutellista 518

Scutovertex 708, 720

Scydmaenus 391

Scydmoraphes 697

Scyliorhinus 808

Scyllarus 710

Scymnus 110, 217, 219, 232, 498, $582,603,680$

Scythris 370, 492, 562

Scythropia 357

Scytodes 444, 463

Seabrina 251, 666

Seeboldia 382

Segestria 444, 538

Sehirus 125, 208

Seira 461, 488, 516, 576

Selagia 229, 382, 545

Selagion 229

Selania 405

Selatosomus 88

Selence 112

Selenia 170, 467

Selidosema 61, 170, 228, 492, 548

Sellnickochthonius 760

Selysiothemis 264, 574

Semanotus 746

Semblis 614

Semiadalia 571, 603, 680

Semidalis 591, 618, 686, 806

Semiothisa 170

Semiothissa 492

Semohemerobius 747

Sepidium 100, 341

Sergestes 619

Serica 214

Sericoderus 63, 630

Sericomyia 231

Sericomyrmex 634

Sericostoma 494

Sericothrips 609

Serinetha 501

Serranus 808

Serratoppia 720

Serromyia 632, 664

Serropalpus 88

Sertella 807

Serviformica 457

Sesamia 98, 143, 228, 299, 427 $468,508,524,549,557,560$

Sesia 74, 113, 225

Sesias 41

Setariola 600

Setina 95, 158, 549

Setodes 494

Shaeroderma 71

Shalbergella 163

Siagona 100

Sialis 46

Sibinia 100, 219

Siculobata 708

Sicyonia 619

Sidemia 98, 430
Sideridis 560

Sideris 61

Sigara 682

Sigilla 416, 458

Silisoptinus 748

Silo 494

Silpha 15, 92, 100, 107, 179, 267,

$338,456,511,551,570$

Silvanus 308

Simaethis 370, 549

Simosa 537

Simpherobius 215

Simulium 606

Simyra 98, 560

Sinaphe 409

Sinella 461

Singa 688

Singilis 697

Sinodendron 88, 214

Sinoxilon 120

Sinoxylon 100, 120, 183, 269 484, 723

Siona 170

Sipalus 506

Sipha 555, 571

Siphocorine 555

Siphonactinia 803

Siphoninus 232

Siphonoperla 707, 788

Siphonophora 485, 555

Sira 461

Sirex 183, 303, 372

Sisyphus 139, 237, 691

Sisyra 618

Sitaris 28, 58, 71, 138, 218

Sitarobrachys 28, 138

Siteroptes 445

Sitochroa 382

Sitona 92, 96, 140, 391, 399, 420 681,751

Sitophilus 119, 308

Sitotroga 301, 405

Sloperia 61, 101, 315, 386, 409 549

Smerinthus 41, 61, 76, 101, 336 , $492,509,545,549$

Smicromyrme 127, 222, 365 $416,442,458,713$

Sminthurides 461, 474, 488, 535 576

Sminthurinus 461, 535, 576, 613

Sminthurus 461, 474, 488, 535

Smithistruma 634

Smittia 46, 565

Smittina 807

Smittoidea 807

Smycronyx 140

Smynthurodes 485, 555

Smynthurus 535, 461

Sobarus 290

Sobula 223

Solea 808

Solen 800

Solenius 608

Solenobia 86, 382

Solenocera 619,710

Solenopsis 39, 455, 457, 543, 634

Solenostethium 125, 208

Solierella 129, 651, 684

Solva 223

Somatochlora 264

Soosia 761

Sophronia 405

Sophronica 290

Sophroniella 290
Sorex 113

Soronia 217, 321, 512, 570

Sospita 649, 680

Spaelotis 95, 560

Spaniotoma 532, 565

Spanioza 232

Sparganothis 9, 405, 548, 549

Sparus 808

Spatalia 90, 190, 643

Spathocera 208, 501

Spatula 588

Spectrobates 382, 406

Speia 557

Spelaeacritus 723

Spelaeobythus 360

Speleosiro 517

Speocharis 528, 724

Speogeus 724

Speonomus 27, 82, 360, 724

Speophilus 360, 362

Spex 671

Sphaenophorus 391

Sphaericus 119

Sphaeridia 576

Sphaeridium 570, 699, 762

Sphaerites 600

Sphaerius 600

Sphaerochthonius 419, 720, 760

Sphaerodema 506

Sphaeroderma 219, 260, 446

Sphaerolecanium 251, 650

Sphaerophoria 231, 309, 394, 500

Sphaerosoma 217

Sphaerozetes 720

Sphalerocoris 602

Spharganothis 198

Sphecapata 365

Sphecia 225

Sphecius 129

Sphecodes 289, 654

Sphedanolestes 176, 602

Sphemura 290

Sphenarium 203

Spheniscomyia 116

Sphenoptera 219, 681, 697

Sphenura 290

Sphex 129, 250, 572, 608, 621, Stenodes 198, 405, 492, 545

$654,684,685$

Sphincterochila 768

Sphinctomyrmex 634

Sphinctus 157

Sphingonotus $345,614,672$, 706,716

Sphintherops 427

Sphinx 101, 225, 236, 302, 429

Sphodrocoris 602

Sphodromantis 318

Sphodrus 38, 110

Spialia 101, 229, 315, 386, 409 $492,548,549,712$

Spilalonius 602

Spilarctia 545, 560, 580

Spilister 723

Spiloconis 806

Spilogaster 365

Spilomena 652

Spilonicrus 489

Spilonota 198, 405

Spilosoma 95, 158, 549, 560, 580

Spilostethus 125, 208

Spilothyrus 19, 37, 74

Spinolia 129, 513

Spiralla 220

Spiris 580

Spirula 619

Spodoptera 98, 157, 316, 384 398, 420, 443, 468, 492, 524, $545,546,548,549,560,623$, 657, 690, 711

Spondiliaspis 232

Spondyliapsis 506

Spondylis 746

Spongelia 800

Spongia 800

Spongionella 800

Spriris 560

Spudaea 61, 98, 560

Spudastica 263, 274, 336

Squamapion 733

Stactobia 494

Stagetus 435

Stagmatophora 370, 548

Standfussia 86, 382

Standfussiana 95, 560

Stangeia 382

Staphilinus 15

Staphylinus 140, 321, 681

Stathmopoda 370

Staudingeria 382

Stauroderos 614

Stauropus 90, 563, 643

Steatoda 755

Steganacarus 419, 720, 760

Stegobium 110, 119, 435, 484

Stegodyphus 486

Stegomyia 4, 97

Stegomyrmex 634

Stegophylla 232, 460, 485

Stelis 129, 289, 637

Stemmatophora 382, 545

Stempellina 46, 565

Stempellinella 565

Stenamma 634

Stenaxis 818

Steneotarsonemus 346

Stenister 723

Stenobothrus 250, 345, 614, 706, 727, 737

Stenocephalus 125, 501

Stenocerus 290

Stenocoris 506

Stenodontes 163, 290, 506

Stenodynerus 584

Stenognathellus 461

Stenohelops 341, 373

Stenolaemus 602

Stenolechia 405

Stenolemoides 602

Stenolemus 602

Stenolophus 38, 96, 110, 160, $359,391,631,700$

Stenomutilla 416, 458

Stenopachys 183

Stenophylax 494

Stenopoda 602

Stenopsocus 589

Stenopterapion 733, 75

Stenopterus 391, 630 


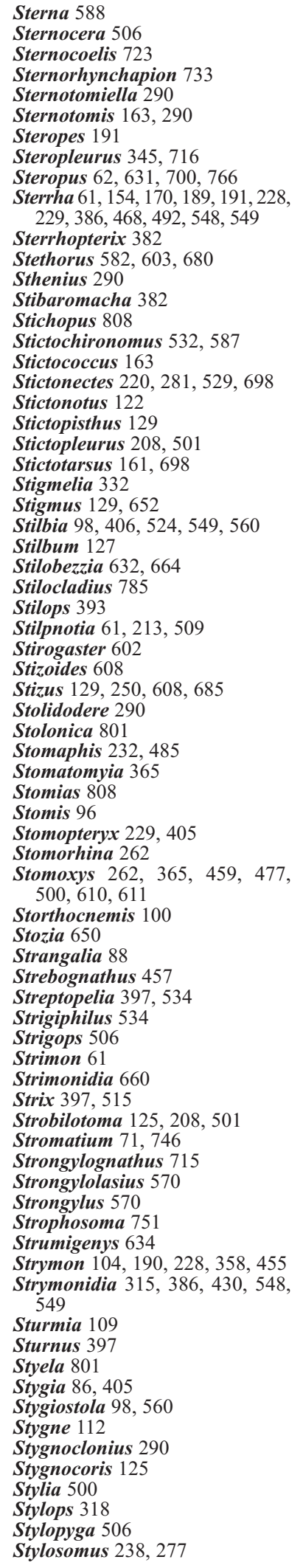

Styphloderes 815

Styphrus 723

Suarius 686

Subacronicta 524

Subcoccinella 582, 680

Subepiblema 198

Subiasella 720

Subisotoma 461, 763

Suboestophora 76

Subpentagona 332

Subtobelba 720

Suctobelba 419, 720

Sudalus 501

Suillia 756

Sula 397, 588

Sulcopolistes 296, 683

Sulcoptinus 748

Sumelis 290

Sundaptinus 748

Swammerdamia 370

Syllyptria 430

Syllythria 382, 492, 545, 548, 549

Sylvicola 477

Symbiotes 63, 217

Symmoca 229, 382, 545

Symmocoides 382,386

Symmorphus 584, 683

Symopherobius 232

Sympecma 396, 647

Sympetrum 264, 396, 647

Sympherobius 22, 590, 591, 618, 686, 736

Symphodus 808

Symphurus 808

Symphya 193

Sympotthastia 532, 785

Synaema 621

Synagapetus 796

Synalpheus 619

Synanthedon 61, 86, 316, 562

Synaphe 228, 229, 382, 430, 492, 548, 549

Synapion 733

Synaptola 290

Synchiropus 808

Synchita 456

Synchloe 29, 104, 195, 315, 316. 386,526

Synchthonius 720

Synclisis 22

Syncopacma 405

Syncricotopus 565

Syndemis 198, 405, 562

Syndiamesa 785

Synechostictus 96, 631

Syneda 61, 98

Synergus 668, 669

Syngrapha 98, 427, 560

Synharmonia 582, 603

Synoicum 801

Synopsia 61, 170, 549

Synorthocladius 565

Syntarucus 104, 228, 315, 409 $410,430,509,548,549$

Syntaurucus 656

Syntomus 62, 96, 219, 220,631

Synthymia 98, 560

Synuchus 62

Synupserha 290

Synvaleria 98

Sypnupserha 290

Syrdenus 92, 110, 322, 412

Syria 382

Syrichthus 660

Syrichtus 629, 712

Syrinus 723, 817
Syritta 309, 394, 500

Syromastes 208, 501

Syromastus 501

Syrphoctonus 319

Syrphus 231, 232, 274, 309, 394. 500

Syssphinx 406

Systenocerus 88, 390

Systropha 289, 654

Syzeuctus 336, 667

Tabanus 177, 459, 554, 586

Tachina 204, 365, 812

Tachinus 630

Tachycines 318

Tachyporus 630

Tachys 38, 96, 700

Tachysphex 129, 138, 201, 250, $458,621,651,684,685,713$

Tachyta 62

Tachytes 129, 651, 684, 685

Tachytila 336

Tachyura 96, 391, 631, 697

Tadorna 588

Taeniapion 733

Taeniocampa 560

Taeniopterix 282

Taeniopteryx 707

Taeniothrips 615

Taitaia 602

Takecallis 485, 555

Talaridris 634

Taleporia 86,382

Talla 650

Tanatophilus 511

Tanca 736

Tanymecus 391

Tanypus 24, 32, 46, 532, 565,

$\begin{array}{cl}\text { 752, } 785 & \text { Tetraphis } 485 \\ \text { Tanytarsus 42, 46, 532, 565, 587, } & \text { Tetrastichus 636, } 656\end{array}$ 717,785

Taphrotopium 733 $645,656,715,719,723,725,802$

Tarache 61, 98, 155, 316, 549

Taragama 8, 41, 73, 101, 113, 409

Targaspidiotus 251

Targionia 251, 633

Tarphius 107, 110, 140, 280

Tarsonemus 346, 445

Tarsostenus 183

Tartarogryllus 706

Tarucus 19, 104, 315, 316

Tates 193

Tathorhynchus 409, 560

Tathorrhynchus 427

Tatuidris 634

Taurotragus 290

Tavaresiella 485, 555

Tecmerium 370

Tectocepheus 419, 488, 708, 720

Tegenaria 26, 226, 503, 538

Tegeocranus 720

Tegostoma 382

Teichobia 357

Tejenaria 26

Teleiodes 405

Teleiopsis 405

Telema 444, 503, 538

Telenomus 711

Telephila 405

Teleproctophylla 22

Telesilla 560

Telmatobius 809

Telmatoscopus 610

Telopelopia 703

Telphusa 405

Thecabius 485, 555
Temnochila 107, 321, 630

Temnoscelis 290

Temnothorax 719, 72

Templetonia 461, 516, 535, 576

$318,321,477,506,603$

Tenebrioides 630

Tenebroides 119

Tenthredo 476

Tentyria 47, 92, 96, 106, 120, $341,373,412$

Tephrina 170, 229, 467, 549

Tephritis 500, 653

Tephrochlamys 756

Tephronia 170, 492, 545, 549

Teracolus 80

Terapha 208

Teratopsocus 589

eredus 484

Terekia 588

Teretriosoma 110

Teretrius 723

Termes 506, 589

Tersilochus 635

Tessellana 345

Tethea 405, 548, 549

Tetrablemma 444

Tetracanthella 461, 474, 516

Tetragonoschema 115

Tetragyropus 502

Tetralobus 506

Tetralonia 320, 395, 617

Tetramorium 33, 634, 715, 719, 725,802

Tetraneura 485, 55

Tetranychus 346, 445, 611, 626

Tetrastichus 636, 656

Tetratoma 600

Tetrix 345,614

Tettigonia 345, 614, 621, 706

Teuchocnemapion 733

Teutonia 687

Thais 8, 12, 41, 113, 302

Thalassomyia 46

Thalassophilus 568

Thalera 170, 228, 229, 549

Thalpobia 100

Thalpochares 430

Thalpophila 98, 524, 560

Thalycra 512, 570

Thamnacarus 419

Thanaos 315

Thanasimus 183

Thanasinus 321

Thanathophilus 219

Thanatophilus 681

Thanotophilus 338

Thatophilus 179

Thaumatomyrmex 634

Thaumatopoea 61

Thaumetopoea 41, 45, 56, 80, 90, $113,189,190,213,302,316$, $403,406,509,545,548,549,583$

Thea 456, 582, 603, 649, 680

Theba 757, 768, 782

Thecla 19, 37, 104, 190, 315, 455, 492, 548

Thelaxes 232, 485, 555

Theleproctophylla 591

Thelychaeta 262

Theobaldia 11, 97, 162, 627

Theocris 290 
Theorectes 92

Theotima 444

Thera 170

Therapha 501

Theraphosa 444

Thereva 365

Theria 170

Theridion 503, 538, 755

Theridium 463, 486

Therioaphis 485

Theroscopus 577

Thersilochus 635

Thestor 19, 61, 93, 102, 113

Theticus 290

Thienemanniella 565, 703

Thienemannimyia 532, 565

Thiodia 198, 405, 562

Thiodiodes 198, 405

Thiotricha 405

Thisanotia 382, 549

Tholera 61, 95, 524, 560

Thopalizarius 290

Thoralus 619

Thordisa 770

Thorectes 681, 691

Thorictodes 600

Thorictus 110, 600

Thormisidae 538

Thoryctus 100

Thoryobothrips 638

Thremma 494

Thrips 44, 615

Thrixion 204

Throscus 219, 391, 600

Thryogenes 391

Thryptera 100

Thumatha 560, 580

Thyas 687

Thyatira 90, 405

Thymalus 88

Thymapion 733

Thymelicus 19, 101, 154, 229, 315,

$316,430,549,660,712$

Thyphaeola 630

Thyphoea 119

Thyphoeus 681

Thypoeus 691

Thyreocerus 608

Thyreonotus 345, 614, 621, 706

Thyreophora 179

Thyreopus 608

Thyreus 320, 617

Thyria 560, 580

Thyridanthrax 500

Thyris 086, 90, 405, 409

Thyrisoma 720

Tibicen 506

Tilapia 544

Tillus 183

Timandra 468

Timarcha 15, 92, 110, 146, 681

Timea 770

Tinea 70, 179, 191, 298, 332

Tineina 406

Tineola 70, 179, 332, 371, 492

Tingimyrmex 634

Tinna 602

Tinocallis 485

Tinodes 494

Tiphia 127, 617

Tiphys 687

Tipula 192, 532, 565, 587

Tischeria 332

Titanio 382

Titanosiphon 798

Titthaspis 680
Tituboea 260, 456

Toltecia 757

Tomares 104, 315, 316, 407, 409, $545,548,629$

Tomocera 518

Tomocerus 46

Topeutis 370

Topobates 720

Torneuma 697

Torpedo 808

Tortricodes 198, 405, 54

Tortrix 198, 213, 263, 298, 302

$316,333,376,405,430,548$

Torymus 636

Toulgoetia 170

Toxocampa 98, 548, 549

Toxolea 193

Toxoptera 232, 485, 670

Trabutina 251, 666

Trachea 98, 549, 560

Tracheliodes 608, 651

Trachilium 225

Trachinus 808

Trachusa 289, 637

Trachylepidia 551

Trachymolgus 445

Trachymyrmex 634

Trachynotus 179

Trachyphloeus 391

Trachys 219, 456

Trachyscelis 47, 100, 110, 120, 291, 341, 413

Trachysmia 198, 405

Trachythrips 638

Tragocephala 163, 290

Tragon 290

Tranopelta 634

Trechocorys 666

Trechus 38, 62, 96, 107, 110 $140,360,568,631$

Tremex 303

Trepanes 96, 631

Trhypochthonius 419, 720

Triacanthella 461

Triaena 461

Triaenea 488

Triaenodes 494

Trialeurodes 232

Triaxomera 332

Tribalus 723

Tribelocephala 602

Triboleum 140

Tribolium 47, 100, 110, 119, 120 308, 318, 506

Tribrachys 551

Trichadenotecnum 589

Trichapion 733

Tricheremaeus 720

Trichia 768

Trichilium 225

Trichiura 61, 101, 157, 190, 228 , 545

Trichius 214

Trichocladius 565

Trichoclea 95

Trichoconapion 733

Trichodectes 397, 502

Trichoderes 506

Trichodes 100, 183, 241, 268

Trichodromius 530

Trichogramma 33, 656, 711

Tricholeiochiton 494

Tricholespis 506

Trichomma 302, 667

Trichoniscus 674

Trichophaga 70, 179, 332, 371, 549
Trichoplusia 468, 492, 546, 578, Truncatella 750

711,560

Trichopodus 110

Trichopsocus 589

Trichopterapion 733

Trichorhina 674

Trichoribates 708, 720

Trichoribatula 720

Trichoscapa 634

Trichosea 427, 546, 560

Trichotanypus 24, 565

Triclistus 263

Trictena 506

Trididemnum 801

Trigla 808

Trigliphothrix 634

Trigloporus 808

Triglyphus 797

Trigona 543

Trigonaspis 653

Trigonidium 345, 706

Trigoniomachilis 575

Trigonoceps 573

Turbinoptes 445 $545,549,560$

Trimalaconothrus 419, 720

Tringa 397, 534, 588

Trinoton 397, 588

Triodia 357, 430, 545

Triops 536

Trioxys 67

Trioza 232, 499

Triphaena 61, 95, 549

Triphleps 125

Triphosa 170

Tripiloppia 593

Triplax 88, 199

Tripoxylon 250

Trisateles 560

Trisopterus 808

Trissexodon 768

Trissocladius 32,785

Trithemis 264, 574, 647

Trithena 444

Tritia 419, 720

Tritoma 88, 199

Tritophia 643

Trixoscelis 756

Trizetes 419

Trochantodon 374

Trochilia 225

Trochilium 225

Trochoidea 768, 782

Trochoideres 217

Troctes 589

Trogaspidia 416

Troglocharinus 360, 362

Troglopedetes 461

Troglops 363

Troglorites 62, 82

Troglorrhynchus 568

Trogocharinus 27

Trogosita 78, 100

Trogoxylon 343

Trogulus 517

Trombicula 445

Troodontella 214

Tropaea 2, 283, 406, 424

Tropidacris 203

Tropidodynerus 584

Tropidonotus 264

Tropidopola 345

Tropidotilla 458

Tropinota 92, 100, 186, 214

Tropocyclops 537

Trox 179, 186, 237, 267, 600

Tryonimus 666

Trypeta 500, 577

Trypocopris 691

Trypodendron 512

Trypopitys 324

Tuberculatus 232

Tuberolachnus 485

Tubifera 477

Tubifex 606

Tubuliferola 370

Turanana 48

Turania 19

Turbicellepora 807

Turnix 113

Txypoxylon 129

Tychea 485

Tychius 100, 733, 751

Tychobythinus 539

Tychus 110

Tylopsis 345

Tymelicus 548

Typhaea 456, 630

Typhlocyba 336

Typhlomyrmex 634

Typhoeus 186, 237

Tyria 158, 492, 548

Tyrolichus 445

Tyrophagus 445

Tyrrhenoleuctra 707

Tyto 534

Tytoniella 534

Tytthaspis 582, 649

Uhleria 666

Uleiota 308

Ulidia 621

Ulmicola 501

Uloborus 486

Uloma 88

Umbonula 807

Umbrina 808

Umbronia 506

Unaspis 633

Unio 647, 786

Unionicola 687

Upupa 534

Upupicola 534

Uranoscopus 808

Uranotaenia 754

Truncatellina 757,782

Truxalis 345, 614, 672, 716

Trymosternus 171, 219, 322, 325,

Tryphon 129, 274, 336

Trypoxylon 572, 651, 684, 685, 713

Tuberculoides 232, 485

Tullbergia 461, 488, 535, 613, 743

Typhlodromus 346, 445

Tyroglyphus 21, 179, 445

Tyta 492, 524, 546, 560

Udea 228, 298, 382, 420, 430, 548

Ulotricha 228, 382, 430, 545 


\begin{tabular}{|c|c|}
\hline Urothemis 264 & Vitrea $729,757,782$ \\
\hline $\begin{array}{c}\text { Ustocidalia } 170,228,298,429 \\
430,468,492,545,548,549\end{array}$ & $\begin{array}{l}\text { Vitula } 382 \\
\text { Viverra } 113\end{array}$ \\
\hline $\begin{array}{l}\text { Utetheisa } 61,95,191,298,316 \\
\quad 409,468,548,560,580\end{array}$ & $\begin{array}{l}\text { Volucella 231, 309, } 394 \\
\text { Volvoxis } 570\end{array}$ \\
\hline Utethesia 549 & Wagnerium 733, 751 \\
\hline Uzelia 461, 474 & Walckenaera 444 \\
\hline Vachiria 176,602 & Wasmannia 634 \\
\hline Vacuna 485 & Weberia 365 \\
\hline Vadenia 405 & Wesmaelius 590, 591, 618, 686 \\
\hline Vaghia 419,720 & Wettina 687 \\
\hline Valdovecaria 382, & Willemia 461, 735 \\
\hline Valenciennellus 808 & Willowsia 461 \\
\hline Valeria 468, 560 & Witlesia 382,545 \\
\hline Valgus 186, 214, 630 & Wormaldia 494 \\
\hline Vallonia 757,782 & Wyeomyia 754 \\
\hline Vanderlindia 731 & Xanthia 560 \\
\hline Vanellus 397, 588 & Xanthochroa 306,818 \\
\hline Vanessa $16,37,41,45,61,68,76$, & Xanthochroina 306 \\
\hline $89,104,112,135,137,229,236$ & Xanthocrambus 228, 382 \\
\hline $315,316,384,386,410,430$, & Xanthodes 492, 545, 560 \\
\hline $432,433,453,509,526,545$, & Xanthoecia 98, 549, 560 \\
\hline $548,549,629,660,676,694,712$ & Xanthogramma 309, 394 \\
\hline Varus 602 & Xantholeuca 61,98 \\
\hline Vasates 346 & Xantholinus 436, 681 \\
\hline Velia $125,131,682$ & Xanthomus 120, 341, 413 \\
\hline Velinea 800 & Xanthoperla 707 \\
\hline Velleda 290 & Xanthorhoe $130,155,170$, \\
\hline Verachthonius 720 & 211, 229, 467, 492, 545, 548, \\
\hline Verlusia 208, 501 & 549 \\
\hline Vermileo 177 & Xenhyboma 767 \\
\hline Veromessor 634 & Xenillus 419, 708, 720 \\
\hline Verongia 800 & Xenochlorodes 170 \\
\hline Vertagopus 461 & Xenomyrmex 634 \\
\hline Vespa $15,33,175,183,296,318$, & Xenonychus 723 \\
\hline $\begin{array}{r}365,506,608,671,683 \\
\text { Vesnorus } 71\end{array}$ & Xenopsylla 50 \\
\hline $\begin{array}{l}\text { Vesperus } 71,681 \\
\text { Vespinitocris } 290\end{array}$ & Xenorchestes 110 \\
\hline $\begin{array}{l}\text { Vespinitocris } 290 \\
\text { Vespula } 296,506,683,765,780\end{array}$ & Xenoschesis 704 \\
\hline Vespula 296, 506, 683, 765, 780 & Xenostrongylus $110,512,570$ \\
\hline Vibertia 602 & Xenylla 461, 474, 516, 535, 576, \\
\hline Vibertiola 602 & \\
\hline Vibidia 582, 649, 680 & Xenyllodes 461 \\
\hline Victrix 524, 546 & Xeris 275 \\
\hline Vietapion 733 & Xerix 303 \\
\hline Viminia 524 & Xeroplexa 768 \\
\hline Vinciguerria 808 & Xerosecta 768,782 \\
\hline Vipera 113,794 & Xerotricha 768, 782 \\
\hline Vipio 193 & Xestia 468, 545, 560 \\
\hline Viteus 485 & Xestobium 321, 324, 435 \\
\hline
\end{tabular}

Xestophanes 668

Xestus 107,110

Xiletinus 324

Xilocopa 33

Xiphuleus 689

Xiphydria 303

Xyela 303

Xyleborus 163

Xylena 560

Xyleutes 506

Xylina 61, 98, 546

Xylina 61,98
Xylion 239

Xylion 239
Xylita 321

Xylobates 720

Xylocampa 560
Xylocopa $129,175,183,289$, $320,506,552,617,758,780$

Xylodes 748

Xylodrema 511

Xylodrepa 338, 511

Xylomania 95, 430

Xylomyia 223

Xylonites 183

Xylopedra 213

Xylopertha 120

Xyloperthella 239, 484

Xyloperthodes 239

Xylorhiza 290

Xylota 231

Xylotrechus 88, 290, 391, 630

Xylotrupes 506

Xysta 204

Xystrocera 290

Yezabura 232, 485

Yola 77, 161, 698

Yponomeuta 263, 370, 406, 545, 548, 549

Ypsolopha 562

Ypsolophus 357

Zabrus 38, 71, 92, 110, 220, 412

Zacryptocerus 634

Zamacra 467, 492

Zanclognatha 98, 560

Zargus 110

Zariquieya 82

Zavrelia 46

Zeaphagus 382

Zebeeba 524, 545, 560
Zegris 8, 12, 61, 93, 102, 104,

$113,135,315,407,430,492$, $526,549,659,694$

Zeiraphera 198, 405

Zele 127

Zelleria 370

Zelotherses 405

Zenobiella 768

Zenodochium 370

Zephyrus 19, 258

Zernyia 170

Zerynthia 41, 61, 93, 102, 104, 156, 194, 315, 358, 406, 430, $454,526,548,549,660,712$

Zeryntya 12

Zetes 720

Zethes 98, 386, 560

Zetorchestes 419, 720

Zeuneriana 614

Zeus 808

Zeuxia 489

Zeuzera 61, 76, 86, 405, 506, 548,549

Zicrona 125, 208

Zilla 688

Zinckenia 299

Zizeeria 315, 316, 644, 660

Zizera 19, 37, 104, 136, 228, 492, 508

Zographus 290

Zonabris 58

Zonites 729, 76

Zonitis 58, 138, 200, 218, 456, 681

Zonitoschema 138

Zonocerus 163

Zonosoma 467

Zoote 158

Zophodia 382

Zophosis 100, 110, 291, 412

Zoropsis 503

Zuphium 96

Zygachipteria 720

Zygaena 8, 37, 61, 68, 113, 189 197, 229, 302, 366, 386, 405, 430, 431, 492, 508, 544, 545, 548,549

Zygonix 264

Zygonyx 574

Zygoribatula 419, 708, 720 


\section{ÍNDICE DE FAMILIAS}

Se relacionan todas las familias mencionadas expresamente en los artículos, tal como lo fueron y con independencia de su consideración actual en cuanto a validez taxonómica. Desafortunadamente, en bastantes trabajos no se indican las familias a las que pertenecen los taxa tratados, y aunque los elaboradores del índice han paliado en contados casos la situación mediante su propia experiencia y conocimientos, no se ha intentado en absoluto completar esta información. El lector interesado en el nivel familiar debiera complementar esta relación acudiendo al exhaustivo índice de géneros que se ha compilado.

\begin{tabular}{|c|c|}
\hline $\begin{array}{l}\text { Acanthiidae } 208 \\
\text { Acaridae } 445\end{array}$ & $\begin{array}{l}\text { Anthophoridae } 28,288,320 \text {, } \\
531,617\end{array}$ \\
\hline Accipitridae 515, 534, 573, 588 & Anthribidae 88, 321, 600 \\
\hline Acentropidae 405, 481 & Anthroceridae 80, 90, 154 \\
\hline Achilidae 151 & Anthrocoridae 215 \\
\hline Achipteriidae 720 & Aphelacaridae 720 \\
\hline Aclerdidae 650 & Aphelinidae 232, 351, 518 \\
\hline $\begin{array}{l}\text { Acrididae } 69,138,163,203,204, \\
226,250,345,350,366,369, \\
443,543,558,611,612,614,\end{array}$ & $\begin{array}{l}\text { Aphididae } 151,190,215,232,396, \\
420,455,460,485,499,555, \\
571,582,649,692,745,779\end{array}$ \\
\hline Actiniidae 803 & $\begin{array}{l}\text { Aphidulade } 84,193,519,5 / 1, \\
\quad 670,711,798\end{array}$ \\
\hline Actinostolidae 803 & Aphodiidae 600, 681, 762 \\
\hline Adelgidae 151, 232, 571 & Apidae $33,58,71,81,129,138$, \\
\hline Adelidae 80, 86, 357, 492 & $165,175,200,201,218,226$, \\
\hline Adelphacaridae 760 & $249,268,289,318,320,395$, \\
\hline Adeonidae 807 & $439,457,458,499,531,543$, \\
\hline Aderidae 163, 600 & $552,617,621,722,758,765,780$ \\
\hline Aegeridae 61, 74 & Apionidae 391, 600, 733, 751, \\
\hline$\underset{549}{\text { Aegeriidae } 80,86,225,314,370,}$ & $\begin{array}{c}772,789 \\
\text { Aplysillidae } 800\end{array}$ \\
\hline Aegialiidae 600 & Aplysinidae 800 \\
\hline Aepophilidae 131 & Aradidae 131, 208, 418 \\
\hline Aeshnidae 396 & Araneidae $82,444,486,503$, \\
\hline Agelenidae 503, 538, 755 & $585,688,755$ \\
\hline Aglycyderidae 110,600 & Arctiidae 41, 61, 68, 74, 76, 80, \\
\hline Agriolimacidae 757 & $89,95,133,154$, \\
\hline$\underset{500}{\text { Agromyzidae } 193,197,337,387,}$ & $\begin{array}{l}158,189,191,213,228,229, \\
236,298,302,371,372,400,\end{array}$ \\
\hline $\begin{array}{c}\text { Agrotidae 61, 80, 89, 95, 98, 143, } \\
154,155,157,169,211,213,\end{array}$ & $\begin{array}{l}429,468,492,509,523,543, \\
545,546,549,580,689\end{array}$ \\
\hline $\begin{array}{l}\text { 492, } 546 \\
\text { Aleyrodidae 151, } 232\end{array}$ & $\begin{array}{l}\text { Arenocoridae } 501 \\
\text { Argynnidae } 89\end{array}$ \\
\hline Algéridos 41 & Argiopidae 486 \\
\hline $\begin{array}{l}\text { Alleculidae } 110,219,321,323, \\
\quad 456,600,630,681\end{array}$ & $\begin{array}{l}\text { Argyresthiidae } 357 \\
\text { Arionidae } 757,775,776\end{array}$ \\
\hline Alpheidae 619 & Armadillidae 462, 674 \\
\hline Alucitidae $370,371,429$ & Armadillididae 462, 674, 675 \\
\hline Alydidae 208, 501, 621 & Arrenuridae 639 \\
\hline Amaurobidae 755 & Ascalaphididae 22, 533, 591, \\
\hline $\begin{array}{l}\text { Amaurobiidae 503, } 538 \\
\text { Ameridae } 419\end{array}$ & 618,686 \\
\hline $\begin{array}{l}\text { Ameridae } 419 \\
\text { Ammotheidae } 594\end{array}$ & Ascidiidae 801 \\
\hline $\begin{array}{l}\text { Ammotheidae } 594 \\
\text { Amorphoscelidida }\end{array}$ & Asilidae 13, 365, 500, 621 \\
\hline $\begin{array}{l}\text { Amorphoscelidida } \\
\text { Amphientomidae }\end{array}$ & Astegistidae 720 \\
\hline $\begin{array}{l}\text { Amphientomidae } \\
\text { Amphisbaenidae }\end{array}$ & Asterolecaniidae 151, 251, 650 \\
\hline $\begin{array}{l}\text { Amphisbaenidae } \\
\text { Anatidae } 588\end{array}$ & Atropidae 589 \\
\hline $\begin{array}{l}\text { Anatidae } 588 \\
\text { Andrenidae } 637\end{array}$ & $0,101,406,426,549$ \\
\hline $\begin{array}{l}\text { enidae } 637 \\
\text { ridae } 418\end{array}$ & Attelabidae 312, 600 \\
\hline $\begin{array}{l}\text { Aneuridae } 418 \\
\text { Anguillidae } 808\end{array}$ & Autognetidae 419, 720 \\
\hline $\begin{array}{l}\text { Anguillidae } 808 \\
\text { Anisopodidae } 72,477\end{array}$ & Axiidae $61,80,90,386$ \\
\hline $\begin{array}{l}\text { Anisopodidae } 72,477 \\
\text { Anisotomidae } 600\end{array}$ & Banksinomidae 720 \\
\hline $\begin{array}{l}\text { Anisotomidae } 600 \\
\text { Annectocymidae } 8\end{array}$ & Bathynellidae 718 \\
\hline $\begin{array}{l}\text { Annectocymidae } 807 \\
\text { Anobiidae } 107,110\end{array}$ & Batrachoididae 808 \\
\hline $\begin{array}{l}\text { Anobiidae } 107,110,1 \\
\quad 180,183,321,324,4\end{array}$ & Belbidae 419, 720 \\
\hline $\begin{array}{l}180,183,321,324,435,456 \\
484,522,600,630,791,821\end{array}$ & Belbodamaeidae 720 \\
\hline Anoeciidae 555 & Beraeidae 494 \\
\hline Anoplocephalidae 419, 445 & Berosidae 648 \\
\hline Anthicidae 96, 100, 110, 138, & Berothidae 22 \\
\hline $\begin{array}{l}140,163,219,456,600,697 \\
762\end{array}$ & $\begin{array}{l}\text { Berytidae 131, } 208 \\
\text { Bethylidae 129, 175, } 457\end{array}$ \\
\hline Anthocoridae 125, 131, 215, 232 & Bibionidae 500 \\
\hline Anthomyidae 179, 489 & Biphyllidae 600 \\
\hline Anthomyiidae 365, 514, 577, 621 & Blastoblasidae 80,370 \\
\hline Anthomyzidae & Blatellidae 706 \\
\hline
\end{tabular}

\section{Blattidae 345, 621 \\ Blenniidae 544, 808 \\ Blepharoceidae 9}

Bómbidos 33

Bombycidae 80, 85, 101, 133, Catantopidae 614, 672, 706, 716 $192,371,406,525,548$

Bombyliidae 347, 500, 621

Borboridae 500

Bostrichidae 71, 88, 100, 108, $110,119,120,163,180,183$, $239,269,321,373,456,484$, $600,630,723$

Bothidae 808

Bovidae 29, 314, 762

Brachodidae 545

Brachycentridae 494, 606

Brachychthoniidae 419, 720, 760

Braconidae 13, 73, 84, 163,175, 193, 232, 286, 314, 337, 367, $375,489,514,571,577,621$, $656,689,765,798$

Brentidae 110, 600, 603, 733

Bruchidae 44, 110, 119,600

Bufonidae 647

Buliminidae 757, 782

Buprestidae 15, 88, 100, 108, $115,119,140,141,180,183$ $219,303,321,434,456,600$ $630,681,697,738,810$

Byrrhidae 600, 738

Bythoscopidae 151

Byturidae 600

Caeciliidae 589

Calamoceratidae 494

Caleremaeidae 720

Caligonellidae 346

Callaphididae 232

Callidulidae 213

Callimorphidae 80

Callionymidae 808

Calliphoridae 78, 179, 262, 365, $477,500,505,506,578,611$, $621,622,626,714,732$

Callistidae 325

Calopterygidae 396, 543

Camillidae 756

Camisiidae 419, 708, 720, 760

Campaea 170

Campichoetidae 756

Canidae 113, 762

Cantharidae 15, 71, 138, 140, $219,321,456,600,68$

Capniidae 282, 606, 707

Caprimulgidae 534

Caproidae 808

Capsidae 125, 131, 163, 232, 420

Carabidae 15, 38, 62, 67, 68, 71 $75,82,88,92,96,100,103$, $107,110,119,120,140,160$, $163,171,173,220,261,321$, $322,325,342,359,391,407$ 412, 466, 470, 489, 530, 568, $600,601,603,630,631,697$ $700,738,762,766,804$

Carabodidae 419, 708, 720

\author{
Caradrinidae 229 \\ Carapidae 808 \\ Carposinidae 80, 198, 254, 405 \\ Castniidae 406 \\ Catopidae 82, 87, 325, 360, 362,
} 456, 511, 528, 568, 724

Cebrionidae 110, 600, 697

Cecidomyiidae 197, 806

Celleporidae 807

Cepheidae 720

Cepolidae 808

Cerambycidae $15,71,88,92$, $100,108,110,119,140,141$, $163,180,183,185,269,290$,

$321,361,391,407,414,450$,

$506,569,600,630,681,697$, $738,746,764$

Ceratopogonidae 9, 459, 585, $632,664,718,777$

Ceratozetidae 419, 720

Cercopidae 39, 151, 621

Cerophytidae 600

Cerylonidae 600

Cetoniidae 163, 600, 681

Chactidae 82

Chaetarthriidae 648, 699

Chaitophoridae 232

Chalcididae 33, 73, 249, 310, 367, 376, 577, 636, 656, 799

Chamaeleontidae 113

Chamobatidae 720

Charadriidae 534, 588

Charilaidae 350

Chaunoproctidae 419

Cheyletidae 346

Chironomidae 11, 24, 32, 34, 42, $46,313,500,532,565,587$, $606,621,665,703,717,718$, $744,752,785$

Chloephoridae 508

Chloroclystis 170

Chloroperlidae 707

Chloropidae 193, 337, 459, 500

Chondrinidae 757, 782

Chorizoporidae 807

Chrysididae 129, 137, 440, 458, $513,572,621,713,820$

Chrysomelidae 9, 15, 71, 78, 88, $92,100,107,110,119,121$, $140,146,147,148,150,163$, 217, 219, 221, 238, 257, 258, $260,266,276,277,278,279$, $304,305,307,312,321,364$, $411,420,456,469,600,649$, 681, 697, 701, 723, 738

Chrysopidae 22, 215, 232, 335, 375, $396,566,590,591,618,686,747$ Chyromyidae 756

Cicadellidae 151, 420, 558, 621

Cicadidae 59, 113, 151, 165, 506, 543

Cicindelidae 15, 71, 75, 92, 110 $113,118,412,600$
Centrolenidae 787 
Cimicidae 66, 131

Cionidae 801

Cisidae 600, 630

Citharidae 808

Cixiidae 151

Clambidae 600

Cleidochasmatidae 807

Cleptidae 347

Cleridae 79, 179, 183, 241, 268, $321,484,600$

Cloropidae 519

Clubionidae 709

Coccidae 13, 136, 151, 163, 215,

$216,232,251,346,364,396$

$498,504,506,518,582,649$ 650,722

Coccinellidae 15, 44, 78, 92, 110

$119,140,165,217,219,232$,

$321,364,456,497,498,518$

$571,582,600,603,649,680$ 681, 738

Cochlicopidae 782

Cochlidionidae 80

Cochylidae 405, 548, 562

Coenagrionidae 396

Coleophoridae 80, 314, 357, 406 , 492, 562

Colletidae 499, 654

Colubridae 113, 264

Columbidae 534

Colydiidae 63, 103, 280, 321, $340,456,484,496,600$ 630

Colymbidae 588

Congridae 808

Coniopterygidae 22, 396, 567, 591, 618, 686, 806

Cononotidae 600

Conopidae 365, 500

Coptosomidae 208

Corduliidae 264

Coreidae 125, 131, 208, 501, 556

Corinetidae 100

Coriscidae 501

Corixidae 131, 506, 682

Corizidae 501

Corvidae 489, 534, 762

Corylophidae 600, 603, 630

Corynetidae 110, 119, 183 484

Cosmetidae 487

Cosmochthoniidae 419, 720, 760

Cosmopterigidae 562

Cosmopterygidae 80, 370

Cossidae 41, 45, 61, 76, 80, 86,

$210,300,371,405,492,525$,

$545,548,549,570$

Crambidae $80,228,229,382$

386, 468, 491, 548, 549

Crangonidae 619

Cribrilinidae 807

Crinopterygidae 80, 332

Crisiidae 807

Cryptophagidae 63, 80, 82, 96, $321,370,456,600,630$

Cryptopidae 620

Crysididae 407

Ctenobelbidae 720

Ctenuchidae 388

Cucujidae 110, 119, $456,600,603,630$

, $9,11,17,34,72$

$97,162,177,330,378,459$

$543,611,626,627,754$

Cupedidae 600
Curculionidae 7, 44, 53, 71, 92, $96,100,103,107,110,119,140$ $141,163,180,183,219,224$, $312,321,325,372,391,399$ $412,420,477,489,531,568$, $600,611,621,626,630,681$, $697,738,751,815,821$

Curtonotidae 756

Cydnidae 125, 131, 208, 621

Cymatophoridae $80,90,263,405$

Cymbaeremaeidae 708, 720

Cynipidae 33, 60, 206, 302, 372,

$439,506,558,577,616,636$,

653, 668, 669, 753, 769

Cynoglossidae 808

Cypselidae 179

Dactylopiidae 151

Damaeidae 720, 795

Damaeolidae 720

Danaidae 56, 385, 543, 544, 646

Dascillidae 600

Dasyceridae 600

Dasytidae 219, 374, 456, 697

Delphacidae 151

Demodicidae 445

Depressariidae 406

Derbidae 151

Dermestidae 69, 87, 100, 110, 179, $321,456,477,510,600,681$

Dexiidae 204

Diapriidae 372

Diaspididae 151, 251, 633

Diastatidae 756

Diastoporidae 807

Dicranocephalidae 501

Dictynidae 755

Didemnidae 801

Dilaridae 22

Dilobidae 524

Diprionidae 558

Dipsocoridae 131

Discoglossidae 744

Discolomidae 603

Disodidae 131

Dolichopodidae 347, 621

Dorilidae 18

Dotoidae 730

Douglasidae 357

Douglasiidae 80

Drassidae 538

Drepanidae 61, 74, 80, 89, 90, $405,409,545,549$

Drepanosiphidae 555

Drilidae 71, 456, 600

Drosophilidae 163, 226, 505, 543, 610, 780

Dryinidae 175

Dryopidae 77, 321, 456, 506, 529,600

Dryptidae 360

Dyctiopharidae 15

Dynastidae 139, 506, 600

Dysderidae 538

Dysideidae 800

Dytiscidae 15, 48, 77, 117, 122

$128,161,184,220,264,281$ $391,506,529,600,698$

Ecnomidae 494

Ectobiidae 706

Elachistidae 80, 229, 357, 559

Elasmidae 375

Elasmodemidae 602

Elateridae 88, 96, 107, 110, 140 $321,391,420,506,600,630$ 681,738

Elipsocidae 589
Elmidae 600, 648

Emesidae 13

Empididae 500, 543

Empodidae 346

Emydidae 113

Enchytraeidae 630, 762

Encyrtidae 18, 215, 251, 364, $375,518,571$

Endomychidae 63, 119, 217, 456, 496, 600, 603

Endromidae 377

Endromididae 382

Enicocephalidae 602

Eniochthoniidae 419, 720

Entomobryidae 461, 474, 488, $516,535,576,613,783$

Epermeniidae 80, 357

Ephialtitidae 765

Ephydridae 262, 621

Epilohmanniidae 419, 720, 760

Epione 170

Epipsocidae 589

Epipyropidae 80, 86, 405

Eremaeidae 720

Eresidae 463

Erestidae 486

Erigonidae 503, 538

Eriococcidae 251

Eriocraniidae 357

Eriophyidae 346, 445

Eriosomatidae 151, 232, 485

Erotylidae 88, 199, 217, 600

Erycinidae 19, 80

Erythraeidae 810

Escharellidae 807

Ethmiidae 80, 155, 314, 370, $492,548,549,562$

Eucinetidae 600

Eucleidae 506

Eucnemidae 88, 321, 600

Eucosmidae 80, 229

Eulohmanniidae 720

Eulophidae 379, 518, 636, 753

Eumastacidae 350

Eumenidae 81, 129, 249, 268, $296,317,318,393,450,513$, $584,671,683,713$

Eupelmidae 518, 636

Euphthiracaridae 419, 720, 760

Eupistidae 80, 357

Euplagiidae 80, 101, 549

Eurytomidae 518, 636, 653

Evaniidae 33, 129, 175, 263, 372

Exochellidae 807

Falconidae 515, 534

Fanniidae 621

Felidae 113

Ferussaciidae 757, 782

Filistatidae 463, 503, 538, 689

Flatidae 151

Forficulidae 344, 345, 706

Formicidae 18, 33, 39, 54, 129 ,

$163,165,175,271,372,373$ $455,457,466,489,543,553$, $570,600,625,630,634,640$, $641,645,650,658,692,695$ $715,723,725,738,765,767,802$

Fringillidae 588

Frondiporidae 807

Gadidae 808

Galleriidae 80, 382, 548, 549

Galumnidae 419, 720

Gamasidae 179, 613

Gammaridae 744

Gasterophilidae 179, 477

Gehypochthoniidae 720

Gekkonidae 264

Gelechiidae 46, 80, 187, 224, 228, 229, 386, 405, 420, 468, $545,548,562$

Geometridae 9, 30, 41, 45, 61, 80,

$113,130,134,154,155,170$,

194, 211, 228, 229, 234, 298,

371, 386, 401, 406, 409, 429,

430, 467, 468, 492, 509, 545,

$546,548,549,630$

Geophilidae 620

Georyssidae 110, 600

Geotrupidae 4, 5, 186, 391, 600, $681,691,762,816$

Gerridae 131, 208, 607, 682

Glaphyridae 600, 661

Glossosomatidae 494, 796

Glyphipterigidae 370, 468, 549,

$$
562
$$

Glyphipterygidae 80

Gnathophyllidae 619

Goeridae 494

Gonodontis 170

Gonyleptidae 487, 517

Gracillariidae 80, 562, 581

Gruidae 588

Gryllidae 226, 243, 265, 345,

420, 443, 506, 543, 614, 621, 706,716

Gryllotalpidae 265, 345, 614

Gymnodamaeidae 720

Gyrinidae 15, 71, 77, 122, 161, 529, 600, 698

Gyropidae 397, 502

Halictidae 621, 637, 654, 713

Haliplidae 122, 161, 529, 600, 698

Haloclavidae 803

Haplochthoniidae 720, 760

Haplozetidae 419, 720

Hebridae 131, 682

Heleomyzidae 179, 756

Helicidae 757, 768, 782

Helicopsychidae 494

Heliodinidae 80, 370

Heliozelidae 80, 332

Helodidae 456, 600

Heloridae 335

Hemerobiidae 22, 232, 335, 396 , 590, 591, 618, 686, 736, 747

Hemileiidae 720

Hepialidae 80, 158, 357, 386, $406,492,506,545,548$

Hermanniellidae 419

Hermanniidae 419

Hesperidae 80, 101

Hesperiidae 19, 37, 61, 74, 89 , 134, 153, 154, 157, 229, 315, 316, 386, 406, 409, 410, 492, $546,548,549,629,660,712$

Heteroceridae 110, 120, 470, 600 
Hydrachnidae 11, 46, 445

Hydraenidae 600, 648, 699

Hydraphantidae 687

Hydriomenidae 229

Hydrobiidae 648, 699

Hydrochidae 600, 648

Hydrometidae 131

Hydrometridae 208, 682

Hydrophilidae 15, 77, 100, 110, $180,456,529,600,603,648,762$

Hydropsychidae 494, 606

Hydroptilidae 494

HydroscAphididae 600

Hydrozetidae 720

Hygrobiidae 122, 600, 698

Hygromiidae 768,782

Hyocephalidae 50

Hypochthoniidae 419

Hypocopridae 600

Hypogastruridae 735

Hyponomeutidae 80, 229

Hypoxistis 170

Hypsidae 229

Ibaliidae 372

Ichneumonidae 6, 13, 73, 129 , 157, 175, 190, 193, 215, 256, $263,272,274,286,302,318$, $319,336,347,372,375,376$, $400,455,457,499,514,577$, $583,621,635,636,656,667$ $689,704,711,722,765$ Incurvariidae 80, 332, 562

Ischyropsalididae 517

Isometopidae 131

Isotomidae 461, 474, 516, 535, $576,613,763,778,805$

Issidae 151

Iwaruna 405

Ixodidae 419, 445

Jassidae 151

Joppeicidae 602

Kermidae 151

Kermococcidae 650

Labiduridae 344, 345, 412, 592, 706

Labiidae 344

Labridae 808

Lacciferidae 151

Lacertidae 721, 738, 739

Lachesillidae 589

Lachnidae 232, 555

Laemobothriidae 397, 515, 573, 588

Laemophloeidae 308

Lagriidae 110, 323, 456, 600

Lampyridae 110, 226, 543, 544, 600,681

Languriidae 600

Laridae 588

Larvaevoridae 179

Lasiocampidae 41, 45, 61, 74, 76,

$80,89,101,133,155,157$

190, 197, 228, 229, 298, 314,

$316,371,372,386,406,409$,

$410,429,430,492,508,509$,

$545,546,548,549,583,630$

Lathiceridae 350

Lathridiidae 63, 87, 110, 119, $321,456,600,630$

Lauxaniidae 365

Lebiidae 171, 219, 325

Lecanidae 251, 650

Lemoniidae 61, 80, 101, 190, 548

Lentulidae 350

Lepidostomatidae 494

Lepismatidae 543
Leporidae 113, 206

Leptestheriidae 536 Leptidae 177

Leptinidae 511, 600

Leptoceridae 46, 494

Leptocoridae 131, 208

Leptodactylidae 809

Lestidae 396

Leuctridae 282, 606, 707, 811

Liacaridae 708, 720

Libellulidae 83, 264, 396, 574, 647

Libytheidae 76, 80, 104, 315, 386, 548

Lichenoporidae 807

Licinidae 325

Licneremaeidae 720

Licnobelbidae 720

Licnodamaeidae 720

Limacidae 757

Limacodidae 80, 86, 386, 405, 468,508

Limnebiidae 456, 648, 699

Limnephilidae 494, 606

Limnichidae 600

Limnozetidae 720

Linmnohalacaridae 718

Linyphiidae 538, 755

Liodidae 511, 630, 708, 720

Liotheidae 502

Liphistiidae 444

Liposcelidae 589

Lithobiidae 620

Lithocolletidae 80, 357, 387, 406, 562

Locustidae 166, 178, 404, 506, 603

Lohmanniidae 419

Lonchaeidae 179, 365

Lophiidae 808

Lucanidae 4, 5, 88, 110, 180, $186,214,390,600,630$ Lumbricidae 630, 762, 781

Lycaenidae 19, 37, 39, 56, 61, 74,

$76,80,89,93,102,104,113$

$134,135,153,154,156,158$ $209,228,229,258,315,316$, $358,372,386,406,408,409$, $410,430,433,454,455,468$, $481,492,507,508,509,545$, $546,548,549,629,644,655$ $656,660,712$

Lycidae 450, 600

Lycosidae 26, 463, 486, 503, 538, 755

Lyctidae 180, 183, 321, 343, 630 Lydidae 303

Lygaeidae 125, 131, 208, 558, 621

Lymantriidae 41, 61, 73, 76, 80,

$98,134,213,314,384,400$ $406,508,509,524,548,549$, $558,630,677$

Lymexylidae 180, 321, 600

Lyonetiidae 80, 332, 548

Macrodiplactidae 264

Macrorhamphosidae 808

Macrouridae 808

Malachiidae 140, 183, 363, 415, 437, 456, 697

Malaconothridae 419, 720 Mantidae 13, 35, 345, 543, 544, 706 Mantispidae 591, 618, 686

Margarodidae 151, 666

Masaridae 129, 249, 296

Megachilidae 637, 713

Megacraspedus 405

Melandryidae 110, 321, 600

Melittidae 617, 637
Meloidae 15, 28, 58, 71, 100, 110

$138,140,183,200,201,218$,

$219,321,456,600,681,772$

Melolonthidae 139, 506, 600, 681

Melyridae 183, 321, 415, 600, 681

Membracidae 39, 107, 151

Menoponidae 397, 534, 588

Merlucciidae 808

Mermithidae 571

Merophysiidae 600

Meropidae 534, 588, 701, 723

Mesembrinellidae 732

Mesophleps 405

Mesopsocidae 589

Mesoveliidae 131, 682

Metrioppidae 720

Metzneria 405

Micreremidae 720

Micromalthidae 321, 600

Microphysidae 131

Microporellidae 807

Micropterigidae 80, 562

Micropterygidae 357

Milacidae 757

Miridae 131, 163

Molgulidae 801

Momphidae 370, 548, 562

Monophlaebidae 15

Monotomidae 308

Mordellidae 100, 219, 456, 600, 630,681

Mullidae 808

Muridae 445, 502

Muscidae 17, 34, 97, 109, 179 $222,262,330,365,420,459$, $477,500,606,611,621,626$

Mutillidae 81, 222, 316, 416, $442,458,489,531,713,722$

Mycetophagidae $63,88,119$ $456,600,630$

Mycobatidae 708, 720

Mycteridae 600, 821

Myctophidae 808

Mydidae 365

Myobiidae 445

Myopsocidae 589

Myriaporidae 807

Myrmeleontidae 22, 293, 396 $590,591,618,686$

Myrmosidae 365, 722

Nabidae 125, 131, 208, 602

Nanhermanniidae 419, 720

Nassidae 81

Naucoridae 131, 682

Neanuridae 735,749

Nebriidae 325

Nemastomatidae 517

Nemonychidae 600

Nemopteridae 22, 591, 618, 686

Nemouridae 282, 606, 707

Neorhacodidae 193

Nepidae 131, 208, 682

Nepticulidae 80, 332

Nettastomatidae 808

Niphocepheidae 720

Nitidulidae 63, 100, 119, 140, 163 $217,321,373,512,551,570,600$

Noctuidae 61, 76, 80, 89, 113, 143 , 157, 190, 191, 197, 204, 228, $236,285,298,299,300,358$, $371,384,386,395,398,400$, $406,409,420,427,429,446$ $467,468,492,508,509,518$ $519,524,525,543,545,546$, $548,549,553,557,561,583$ $621,623,630,657,690,711$

Nolidae 80, 95, 229, 549

Noteridae 161, 600

Nothridae 419, 720

Notodontidae 41, 61, 68, 73, 80, $90,155,157,190,213,402$, 406, 448, 466, 468, 492, 493, $509,545,548,549,563,643$

Notonectidae 131, 208, 682

Nycteolidae 524

Nymphalidae 16, 37, 41, 45, 61, $64,68,74,76,80,89,91,102$, $104,134,135,137,153,154$, $195,228,229,236,315,316$, $358,371,384,385,386,406$, $409,410,430,432,433,450$, $453,454,468,482,492,507$, $508,509,525,526,543,545$, $546,548,549,558,628,629$, $660,676,694,712,759$

Ochodaeidae 600

Ochsenheimeriidae 80, 332

Ochteridae 131, 208, 682

Ochthebiidae 648, 699

Ochyroceratidae 444

Odontellidae 740

Odontoceridae 494

Oecanthidae 614

Oecophoridae 69, 70, 80, 229 $370,386,468,492,545,562$

Oedemeridae 306, 321, 456, 600 , 818,821

Oestridae 179

Omalogyridae 750

Oncopodidae 487, 517

Oniscidae 674

Onychiuridae 461, 474, 488, 535 , $576,613,734$

Opilionidae 487

Oplophoridae 619

Opomyzidae 500

Opostegidae 80, 224, 332

Oppiidae 419, 593, 720

Orgyidae 213

Oribatellidae 419, 720

Oribatidae 419, 718

Oribatulidae 708, 720

Ormyridae 653

Orneodidae 80, 210, 370

Orsodacnidae 600

Ortalidae 477

Ortheziidae 151, 666

Orthoperidae 63

Oryssidae 303

Ostomidae 119, 140, 321

Otididae 705

Otitidae 500

Oxyopidae 538

Oxyuridae 489

Pachylommatidae 175, 193, 263

Pachynomidae 602

Paguridae 710

Palaemonidae 619 
Pasiphaeidae 619

Passalidae 180, 214, 506

Passalozetidae 720

Pectidae 600

Pediculochelidae 760

Pelegonidae 131

Pelobatidae 787

Pelodytidae 787

Pelopidae 720

Pemphigidae 215, 555

Penaeidae 619

Pentatomidae 59, 125, 131, 163 208, 506, 621

Peripsocidae 589

Peristediidae 808

Perlidae 46, 282, 707

Perlodidae 282, 707

Perophoridae 801

Phalacridae 100, 232, 600

Phalaenidae 213, 371

Phalangiidae 487, 517

Phalangodidae 517, 539

Phaloniidae 80, 198, 229, 562

Phasgonuridae 404

Phasianidae 588

Phasmatidae 345

Phenopelopidae 419, 708

Phidoloporidae 807

Philopotamidae 494

Philopteridae 397, 473, 502, 534 588, 705

Philotarsidae 589

Phlaeothripidae 638

Phlebotomidae 459

Phloeomyzidae 555

Phloeophilidae 600

Phloeothripidae 216

Phoenicococcidae 251

Pholcidae 486, 503, 538

Phoridae 128, 179, 347, 477, 57

Phrygaenidae 264

Phryganeidae 481, 494

Phthiracaridae 419, 720

Phycitidae 80, 155, 164, 228, 229, $235,298,382,545,548,549$

Phylloxeridae 151, 232, 485

Phymatidae 131, 602

Phytomyzidae 337

Phytoseiidae 346

Pieridae 37, 41, 61, 68, 73, 80, $89,93,102,104,113,134,135$ $140,157,229,236,314,315$, $358,367,386,406,409,410$ $428,430,433,450,455,468$ $492,509,526,541,545,548$ $549,550,629,659,660,693$ 694, 712

Piesmatidae 131, 472

Pimplidae 765

Pionidae 687

Piophilidae 179, 477

Pipunculidae 500

Pirnodidae 720

Pisauridae 486, 538

Plataspidae 131

Plateremaeidae 419

Platydorididae 770

Platypodidae 163, 180, 312, 321, 600

Pleidae 682

Ploairidae 131

Plusiidae 229

Plutellidae 80, 229, 357, 548, 549

Pneumoridae 350

Podapolipidae 445

Podicipitridae 588
Poduridae 461, 474, 488, 516, $535,576,613$

Polycentropodidae 494

Polycitoridae 801

Polyclinidae 801

Polyctenidae 131

Polydesmidae 82

Pompilidae 441, 450, 486, 531

Porcellionidae 462, 674, 675

Prionidae 600

Processidae 619

Proconiidae 151

Proctotrupidae 335, 372, 489

Prostomidae 600

Proterhinidae 600

Protoplophoridae 720, 760

Protoribatidae 720

Pselaphidae 110, 120, 630

Pselaphididae 103, 360, 456, 539,600

Psephenidae 600

Pseudococcidae 151, 666

Psilidae 193, 649, 784

Psocidae 589

Psoidae 484

Psychidae 56, 73, 80, 86, 157, 336 382, 406, 468, 545, 549, 562

Psychodidae 5, 11, 14, 17, 25, 97 477, 500, 702

Psychomyiidae 494, 744

Psyllidae 151, 232, 499

Psyllipsocidae 589

Pterochthoniidae 419

Pterodelidae 589

Pterolonchidae 80, 370,- 406

Pteromalidae 376, 518, 616, 636, 689

Pterophoridae 74, 80, 209, 210 $228,229,371,382,406,409$, 430, 468, 483, 492, 545, 548

Pterostichidae 62, 67, 82, 119 , $173,325,360,766$

Ptiliidae 88, 456, 600, 630

Ptinidae 87, 88, 110, 119, 175, 219 $360,456,600,630,748,821$

Punctidae 757

Pupillidae 782

Pussidae 600

Pyemotidae 445, 489

Pyralidae 6, 37, 45, 80, 164, 179 $228,229,287,301,371,382$ $386,406,409,420,429,430$, 468, 491, 492, 545, 546, 548, 549, 557, 558, 711

Pyraustidae 80, 155, 228, 229 $298,299,382,409,468,481$, $546,548,549$

Pyrgomorphidae 350, 614, 672 716

Pyrochroidae 321, 600, 630, 818 , 821

Pyrrhocoridae 125, 131, 208, 501

Pythidae 321, 600, 821

Pyuridae 801

Rajidae 808

Rallidae 588

Ranidae 264, 647, 744

Reduviidae 125, 131, 176, 208 $602,611,621,626$

Rhagionidae 500

Rhipiphoridae 321, 600

Rhizophagidae 321, 600

Rhopalidae 501, 621

Rhyacophilidae 494, 606

Rhynchitidae 733

Rhysodidae 103, 600
Ricinidae 397, 588

Riodinidae 80, 89, 104, 315

Rutelidae 600

Saldidae 131

Salpingidae 321, 600

Salticidae 26, 444, 463, 538, 755

Sapygidae 129, 175

Sarcophagidae 179, 365, 477, $500,506,621$

Sarrothripidae 524, 546

Saturnidae 182, 189, 283, 334, 406

Saturniidae 41, 61, 76, 80, 93, $101,123,133,153,163,191$, $371,386,409,468,508,509$, $543,545,548$

Satyridae $12,30,37,61,68,74$ $80,89,104,113,134,154,196$ $228,229,236,273,315,358$ $386,406,409,410,430,492$, $509,545,546,547,548,549$, $558,660,694,712$

Savignyellidae 807

Scaphidiidae 110, 600

Scarabaeidae 4, 5, 28, 31, 69, 75, $88,92,96,100,108,110,119$ $139,163,179,180,186,202$, $214,219,220,226,237,248$, 259, 267, 321, 361, 389, 391, $420,438,477,495,527,559$, $600,603,630,662,663,679$, $681,691,697,738,762,773$, 780

Scariidae 151

Scaritidae 106, 163

Scatophagidae 365, 500

Scelionidae 621, 711

Scheloribatidae 419, 708, 720

Schendylidae 620

Schizoporellidae 807

Schreckensteiniidae 80, 370

Sciaenidae 808

Sciaridae 500

Sclerosomatidae 517

Scoliidae 531

Scolopacidae 588

Scolytidae 13, 110, 140, 141, 163, $180,183,303,312,321,373$, $456,512,600,630,723$

Scophthalmidae 808

Scorpaenidae 808

Scraptiidae 600

Scutelleridae 125, 131, 208, 621

Scutoverticidae 708, 720

Scydmaenidae 63, 103, 391, 600, 697

Scyliorhinidae 808

Scythrididae 80, 187, 370, 492 , 559,562

Scytodidae 463

Selidosemidae 229

Sepsidae 477, 500

Sergestidae 619

Sericostomatidae 494, 606

Serphidae 335

Serranidae 808

Sesiidae 41, 74, 76, 113, 210, $450,545,562$

Silphidae 15, 92, 179, 219, 338 , $456,511,600,681$

Silvanidae 308, 321, 600

Simpherobidae 215

Simuliidae 9, 459, 500, 606, 744

Siricidae 175, 268, 275, 303, 372 , 457

Sironidae 487

Sisyridae 396, 618

Sminthuridae 461, 474, 488, 535 . 613

Smittinidae 807

Soleidae 808

Soricidae 113

Sparidae 808

Sphaeridiidae 648, 699

Sphaeriidae (Bivalvia) 786

Sphaeriidae (Coleoptera) 600

Sphaeritidae 600

Sphaeroceridae 477, 500

Sphaerochthoniidae 419, 720, 760

Sphecidae 33, 81, 129, 138, 175,

201, 250, 268, 289, 318, 365,

$392,471,486,489,499,531$,

$543,572,608,621,651,652$, $684,685,713,722$

Sphincterochilidae 768

Sphindidae 600

Sphingidae 13, 20, 41, 56, 61, 73, 74, 76, 80, 101, 102, 109, 126, $135,190,191,197,210,229$, 236, 316, 386, 409, 468, 480, $492,509,545,548,549$

Spinozetidae 720

Spongiidae 800

Squalidae 808

Squamiferidae 674

Staphylinidae 7, 15, 63, 71, 96, 100, $103,107,110,113,120,140$, $163,179,237,321,360,412$, 436, 470, 600, 630, 681, 697

Steganacaridae 720, 760

Stenocephalidae 501

Stenopsocidae 589

Sternoptychidae 808

Sterrhidae 229

Stichopodidae 808

Stigmaeidae 346

Stigmellidae 80

Stomiidae 808

Stratiomyidae 223, 477, 564

Strigidae 515, 534, 700

Styelidae 801

Subulinidae 757, 782

Suctobelbidae 720

Syllidae 742

Symmocidae 382, 386, 406, 545

Syngnathidae 808

Syntomidae 80, 90

Syrphidae 13, 215, 216, 231, 232, $309,394,450,477,500,621$, 652,797

Syssphingidae 68, 80, 101, 134, $182,316,334,406,468$

Tabanidae $9,46,417,459,554,586$

Tachinidae 73, 109, 179, 204, 347. $365,376,489,500,621,812$

Taeniopterygidae 282, 707

Tarsonemidae 346, 518

Tectocepheidae 708, 720

Telemidae 503, 538 


\author{
Tetragnatidae 755 \\ Tetranychidae 346, 419 \\ Tetraonidae 473 \\ Tetraphaleridae 600 \\ Tetrastemmatidae 813 \\ Tetratomidae 600 \\ Tetrigidae 345, 614, 706, 716 \\ Tettigometridae 151 \\ Tettigoniidae 345, 366, 404, 543 , \\ $603,612,614,621,673,706$ \\ Thaumetopoeidae 41, 61, 80, 90 \\ 403, 406, 509, 545, 548, 549, 583 \\ Thelaxidae 232, 555 \\ Therevidae 365, 500, 621 \\ Theridiidae 463, 486, 503, 538, 755 \\ Thomisidae 538, 621 \\ Thorectidae 800 \\ Thorictidae 110, 600 \\ Thripidae 44, 609, 615, 771 \\ Throscidae 219, 391, 600 \\ Thyatiridae 405, 548, 549 \\ Thyreophoridae 179 \\ Thyrididae 80, 86, 90, 405, 409 \\ Tineidae $70,73,80,179,209$ \\ $332,371,386,407,429,492$, \\ 549,562
}

Tingidae 131, 208

Tingitidae 125

Tiphiidae 457, 722

Tipulidae 519, 606

Tischeridae 80, 332

Torpedinidae 808

Tortricidae 80, 146, 147, 148, 149 ,

$150,198,213,224,228,229$,

$254,270,298,376,380,388$

$405,406,420,429,468,492$,

$519,545,546,548,549,562,630$

Torymidae 636, 653

Trachinidae 808

Trachytidae 808

Travuniidae 517, 539

Trechidae 325, 360

Trhypochthoniidae 419, 720

Triaconichidae 517

Trichiuridae 808

Trichodectidae 397, 502

Trichogrammatidae 711

Trichoniscidae 674

Trichopsocidae 589

Triglidae 808

Tripetidae 421, 423

Trixoscelididae 756
Trogidae 4, 5, 186, 267, 600

Trogiidae 589

Trogossitidae 321, 600, 630

Trogulidae 517

Trombiculidae 445

Trypetidae 116, 233, 372, 387 $447,465,475,477,500,540$

$578,579,595,596,604,610$, $611,622,624,642,653$

Tydeidae 346

Tylencholaimidae 731

Typhlocibidae 151

Tyroglyphidae 179, 531

Tytonidae 534

Uenoidae 494

Ulidiidae 500, 621

Uloboridae 486, 755

Ulopidae 151

Umbonulidae 807

Unionicolidae 687

Upupidae 534

Uranoscopidae 808

Urocteidae 538

Valloniidae 757, 782

Veliidae 125, 131, 682

Verongidae 800
Vertiginidae 757, 782

Vespidae 33, 81, 129, 175, 268 296, 318, 457, 621, 683, 765

Viperidae 113

Viverridae 113

Westermanniidae 80, 95, 228, 298, 299, 508, 524, 549

Xanthonychidae 768

Xenillidae 720

Xiphydriidae 303

Xylophagidae 223

Xyloryctidae 80

Yponomeutidae 370, 375, 406, $464,545,548,549,562$

Zeidae 808

Zetomotrichidae 720

Zetorchestidae 419, 720

Zonitidae 729, 757, 782

Zoropsidae 503

Zygaenidae 37, 45, 61, 68, 74, 80, 191, 229, 386, 405, 406, 409. 492, 508, 509, 525, 544, 545, 548,549 


\section{ÍNDICE GEOGRÁFICO HISPANO-LUSO}

Este índice únicamente contiene información faunística, aunque sea mínima, y no se incluyen las menciones de lugares realizadas por otros motivos en la revista. Se relacionan por separado las provincias españolas, los distritos portugueses, las islas y archipiélagos (únicamente en los casos en que no se mencionan islas concretas) de España y Portugal, Ceuta, Melilla, Gibraltar y Andorra. No se han indexado los pocos casos en que las áreas de distribución se indican únicamente mediante líneas en mapas generales. No se incluyen las referencias sin especificar a España.

Álava 136, 154, 155, 191, 236, $257,268,298,309,389,461$, $485,514,523,555,569,614$ $633,649,650,662,666,682$, $723,727,729,737,768,788$ Albacete 4, 9, 36, 114, 129, 159, $176,213,236,248,249,258$, $267,276,316,336,343,358$, $365,372,395,399,408,433$, $495,507,523,527,551,555$, $561,569,570,571,572,633$, $649,650,652,654,662,668$, $679,682,723,727,737,771$, 789, 795

Alborán 413, 430, 803, 807, 808

Algarve 114, 172, 174, 240, 259,

$345,346,347,399,461,552$, $574,586,662,673,674,682$, 709, 723, 729, 737, 760, 761, $773,775,776,803$

Alhucemas 485

Alicante 4, 22, 25, 36, 121, 129, 143, 157, 172, 176, 191, 204, 213, 218, 234, 235, 248, 249, 250, 259, 262, 263, 267, 299, $302,309,316,319,322,336$, $341,345,365,372,386,392$, 395, 396, 398, 399, 400, 408, $409,412,427,430,440,446$, 454, 461, 468, 469, 472, 485, $492,495,497,507,514,518$, $522,523,527,530,545,551$, $555,564,568,569,570,571$, $572,589,617,633,637,644$, 649, 650, 652, 654, 662, 666, $670,672,674,679,682,686$, 706, 723, 725, 729, 737, 768, $779,801,802,815$

Almería 20, 113, 119, 129, 130, 143, 154, 157, 159, 176, 191, 197, 213, 217, 221, 235, 236, 238, 240, 248, 249, 258, 260, 273, 276, 298, 299, 300, 309, $316,322,336,345,365,372$, $373,374,386,389,392,398$, $399,409,411,412,413,415$, 419, 428, 430, 461, 468, 469, $485,492,495,496,508,514$, $523,527,533,540,546,555$, $557,569,572,574,584,590$ $617,633,635,637,638,649$, $650,654,662,666,668,674$, $679,682,686,693,697,706$, $722,723,725,727,737,758$, $761,768,771,779,780,801$, $802,806,808,814,815$

Alto Alentejo 174, 248, 347, 365, 376, 389, 436, 461, 551, 574, $662,682,723,729,775,776$

Andorra 173, 184, 274, 281, 319, 336, 396, 418, 527, 570, 679, $709,723,766,784,796$

Asturias 9, 62, 78, 80, 129, 153, $154,155,157,158,159,175$, 181, 189, 191, 196, 213, 236,
248, 262, 263, 267, 268, 273, $282,298,308,316,319,323$ $336,365,372,386,392,397$, $399,409,419,427,430,431$, $453,461,467,468,469,473$ $485,492,495,507,514,515$, $523,527,553,554,555,565$, $568,570,572,587,614,633$ $635,637,649,650,652,654$ $662,668,679,682,688,704$ $709,718,724,727,729,737$, 738, 739, 741, 768, 770, 788, 806

Ávila 2, 9, 20, 37, 91, 114, 129, $136,154,156,157,159,176$ $182,191,194,196,208,213$, $236,248,249,257,258,259$ $262,263,267,268,273,274$, $298,302,303,316,319,323$ $334,336,341,365,372,386$, $390,392,397,400,401,402$, $406,415,418,419,430,440$, $454,460,461,468,485,492$ $495,507,508,513,514,523$, $527,534,545,555,565,569$ $570,571,572,575,576,586$ $587,608,609,614,615,617$ $621,633,635,637,649,652$, $654,662,666,668,675,679$, $682,694,699,706,708,709$ $720,721,722,723,726,727$ $728,737,740,743,751,762$ $763,778,779,796$

Azores 110, 236, 280, 291, 391, $524,532,614,619,657,674$ $729,741,750,756,775,801$ 803

Badajoz 4, 174, 213, 232, 236, $248,260,267,268,386,396$ $397,398,399,475,485,527$ $551,555,569,570,584,633$ $637,649,650,665,666,673$ $681,682,699,722,723,737$, 761,806

Baixo Alentejo 174, 347, 365 , 376, 392, 399, 461, 481, 551, $574,655,673,674,675,682$ $700,729,761,775,776$

Baleares 3, 7, 36, 53, 117, 159, 172 , $174,183,214,218,230,250$ $258,264,267,268,298,306$, $307,340,341,365,373,374$ $398,402,412,413,414,435$, $437,440,485,496,522,524$ $529,551,570,571,592,614$ $617,619,637,649,654,674$ $680,682,699,706,723,725$ $741,768,771,779,801,808$

Barcelona 3, 4, 7, 9, 11, 14, 24, 25, $27,32,37,42,48,58,79,83,88$, $91,96,108,117,119,120,122$ $129,154,155,156,157,158$ $159,161,164,172,174,175$, $176,184,185,186,191,199$ 200, 202, 213, 217, 218, 220,
$235,236,248,250,257,258$, $261,263,264,267,273,274$ $281,282,283,298,299,302$ $303,307,308,309,316,319$, $320,323,325,333,336,341$, $360,362,363,365,372,373$, $374,385,386,389,390,391$, $392,393,398,399,400,401$, $402,406,409,413,414,415$, $418,426,429,430,435,437$, $439,440,446,453,454,456$ $461,467,468,482,484,485$, $491,492,495,496,499,507$. $513,514,523,527,532,534$, $537,545,546,551,555,563$, $568,569,570,571,572,577$, $589,617,619,633,636,637$ $638,648,649,650,652,654$, $662,666,668,669,671,674$, $679,682,699,701,704,706$, $709,722,723,727,729,737$ $753,756,766,768,769,783$, $796,801,812,813$

Beira Alta 114, 159, 224, 248, $426,436,461,495,571$, $584,662,679,709,722$, $723,727,729,737,740$ $775,776,796$

Beira Baixa 461, 476, 662, 727 729, 775, 776

Beira Litoral 172, 174, 248, 344 $361,365,375,392,436,461$, $476,495,516,527,551,570$, $574,576,649,662,674,679$, $682,709,723,727,729,737$, $749,775,776,785$

Burgos 62, 111, 129, 135, 137, $154,155,157,189,190,191$, 196, 209, 213, 225, 228, 231, $236,248,249,257,259,267$ $273,282,298,303,316,319$, $336,345,372,386,388,399$, $402,406,408,409,418,419$, $426,427,430,431,433,453$, $454,461,467,468,485,492$ $495,507,508,514,523,527$ $528,545,546,553,561,565$, $570,571,573,587,614,633$ $636,638,649,652,654,662$ $666,679,682,704,718,723$ 724, 727, 729, 737, 741, 768, 786, 788, 813

Cabrera 3, 139, 159, 413, 495

Cáceres 4, 9, 14, 36, 37, 114 $129,176,191,213,230,236$, $248,249,251,260,262,263$, $268,273,276,316,336,365$, $372,386,389,396,397,409$, $461,468,485,492,507,511$, $524,527,534,548,565,569$, $570,571,572,575,584,586$, $587,590,608,617,618,621$ $633,637,649,650,652,654$, $662,666,679,682,684,705$ 709, 723, 729, 737, 769, 806
Cádiz 4, 18, 36, 64, 106, 129, $143,154,155,157,164,172$, $174,176,184,190,191,199$, 200, 205, 206, 208, 213, 214, $219,235,236,240,248,249$, 251, 257, 258, 260, 263, 267, 277, 298, 299, 304, 308, 316, $319,323,336,343,345,365$, 374, 386, 389, 392, 396, 398, 399, 402, 409, 411, 413, 415, 426, 427, 428, 430, 437, 438, $439,440,446,454,461,467$, $468,474,475,484,485,492$, 493, 495, 523, 524, 527, 545, $546,551,555,561,563,565$, 569, 570, 571, 572, 574, 577, 584, 587, 592, 617, 619, 621, 633, 637, 638, 644, 648, 649, $650,652,654,666,674,679$, 682, 684, 693, 699, 706, 722, 723, 725, 737, 753, 761, 768, $769,779,788,800,801,802$, $803,806,807,808$

Canarias 78, 87, 97, 119, 138, 139, 140, 143, 159, 164, 172, 174, 204, 218, 220, 223, 236, $240,251,280,291,298,307$, $317,345,364,396,398,413$, 434, 456, 483, 511, 512, 522, $524,532,565,568,577,587$, $592,600,614,617,619,644$, 648, 649, 657, 674, 675, 686, 701, 708, 712, 714, 723, 725, 733, 737, 750, 756, 757, 771, $779,803,806,807$

Cantabria $4,9,62,76,89,136$, 154, 157, 158, 181, 184, 189, 191, 196, 199, 208, 209, 213, $230,231,236,248,251,257$, $262,263,267,273,282,298$, 303, 304, 308, 316, 319, 323, $336,337,343,365,372,386$, 394, 397, 399, 400, 401, 402, 406, 409, 410, 418, 419, 426, $427,430,433,453,454,461$, 467, 468, 480, 485, 492, 493, 495, 507, 508, 514, 523, 527, $528,532,545,546,553,554$, $565,570,571,587,589,590$, $614,617,619,633,638,649$, $650,652,654,662,666,679$, 682, 709, 718, 723, 724, 727, $729,737,741,743,756,768$, $769,788,796,806$

Castellón 9, 22, 159, 172, 218, 236, 248, 249, 257, 267, 298, $309,316,319,341,360,373$, $386,389,396,398,399,415$, $418,426,461,484,485,495$, $522,523,527,539,545,565$, $568,569,570,587,617,619$, $633,649,650,652,654,662$, $670,674,679,682,706,709$, 723, 727, 729, 737, 765, 768, $779,801,814$ 
Ceuta 48, 77, 174, 230, 277, 363, Girona 3, 9, 14, 25, 27, 29, 58, $438,619,801$

Chafarinas 230, 619

Cíes 461

Ciudad Real 4, 9, 36 53, 114, $129,159,174,176,184,189$ $199,218,221,230,236,240$ $248,249,251,257,267,268$, $276,277,298,302,316,323$ $336,365,372,386,394,396$ $399,400,411,412,437,440$ $461,468,469,474,485,495$ $513,514,523,527,545,551$, $555,565,569,570,571,572$, $587,589,609,615,617,627$ $633,635,636,637,638,649$ $650,652,654,662,666,668$, $673,674,679,681,682,684$ 699, 701, 704, 709, 723, 737, 769,771

Columbretes 159, 341, 412, 419, 461, 522, 706, 709, 801, 807

Córdoba 4, 9, 14, 36, 113, 114, $129,143,154,162,172,174$. $176,213,214,230,236,248$ $251,260,267,268,280,282$ $298,302,304,319,324,336$ $343,365,372,386,389,396$, $398,399,419,440,464,484$, $485,508,514,523,527,551$ $557,565,569,570,571,575$, $587,588,614,617,628,633$, $637,638,644,645,648,649$ $650,652,654,662,674,679$ $681,682,693,695,698,699$ $704,722,723,737,761,779$, 804, 806

Coruña $62,129,154,213,232$ $236,248,257,258,263,268$ $281,282,298,303,319,390$, $392,397,426,430,461,468$ $485,493,507,508,523,527$, $534,553,555,563,565,570$, $575,587,615,617,633,649$ $650,652,654,662,666,674$ $679,682,723,727,729,737$ $761,769,785,788,789,801$

Cuenca 9, 12, 16, 19, 36, 37, 74 $80,93,129,154,155,156$, $176,189,190,196,209,213$ $225,232,236,238,248,249$, $257,267,273,282,298,309$, $316,323,325,336,365,372$ $373,386,389,396,397,399$, $400,402,406,409,426,430$, $433,461,468,484,485,492$, $493,495,507,508,513,515$, $523,524,527,545,546,553$ $555,561,565,569,571,586$, $587,614,617,631,633,637$, $648,649,650,652,653,654$. $660,662,665,666,673,674$ $679,681,682,709,712,722$, $723,727,737,768,771$

Douro Litoral 248, 257, 344, 365 , $392,436,453,461,679,682$ $709,722,723,727,729,775$

Estremadura 174, 232, 240, 268 $344,346,365,375,392,399$, 402, 426, 436, 461, 476, 570, $574,679,682,688,709,722$ $723,729,737,775,776,801$, 806

Formentera 36, 139, 396, 723

Fuerteventura 110, 291，298, $317,442,472,531,667$
$78,82,129,154,155,156,157$ $173,176,184,185,191,199$ $200,208,213,217,228,236$ $240,248,250,258,260,261$ $263,273,274,277,281,298$ $299,302,303,308,309,316$ $319,336,341,360,363,365$ $371,372,374,389,392,393$ $399,400,402,409,413,415$, $418,426,430,437,439,440$ $453,454,458,461,468,481$, $482,485,492,495,499,507$ $523,527,532,545,546,555$ $565,568,570,571,572,577$ $617,619,633,637,638,648$ $649,650,652,654,662,665$ $666,668,671,673,674,679$ $682,699,701,709,723,727$ $783,786,796,801,802,812$

Gibraltar 36, 113, 174, 189, 248 , $298,345,485,492,523,527$ $723,725,729,800,801,802$

Gomera 110, 162, 280, 291, 298 , $434,531,575,747,806$

Gran Canaria 4, 97, 110, 120, 162 , $240,291,317,342,483,485$, $522,531,555,667,747,806$

Granada 4, 36, 37, 78, 80, 111 , $113,114,129,135,154,155$, $156,157,159,171,176,189$ $190,191,196,199,206,208$ $209,213,216,218,221,235$ $236,238,240,242,248,249$ $250,251,258,259,260,262$ $267,268,273,276,277,281$ $282,298,299,302,307,309$ $314,316,319,320,323,336$ $344,345,365,372,374,386$ $389,392,394,396,397,398$ $399,402,406,408,409,415$, $419,426,427,428,430,433$ $437,438,440,446,453,454$ $484,485,492,495,497,498$, $507,508,514,518,523,527$ $532,534,545,546,555,557$ $561,565,566,569,570,571$, $572,575,576,577,584,587$ $589,609,615,617,619,633$ $637,638,644,648,649,650$ $652,654,655,662,665,666$ $668,674,679,681,682,697$ 699, 701, 709, 717, 723, 725 $727,729,737,745,769,779$ $786,788,802,806,807,808$

Guadalajara 4, 9, 61, 129, 154 $176,191,213,214,232,236$ $248,251,262,267,268,273$, $282,298,316,323,365,372$ $373,397,399,400,402,409$, $419,440,461,468,485,492$, 493, 495, 507, 513, 514, 523, $527,545,550,551,555,561$, $565,571,572,575,587,590$ $617,631,633,637,649,650$ $652,654,656,660,662,665$ $666,668,674,676,679,682$ $689,712,723,727,729,734$ $737,759,763,771,778,785$ $788,789,806$

Guipúzcoa 9, 62, 129, 154, 191, $196,213,236,248,262,273$ 283, 298, 309, 319, 323, 336. $585,587,589,594,609,615$ $729,737,753,756,766,768$ $461,464,467,468,472,474$
$365,386,402,403,409,426$, $431,461,485,492,507,527$ $555,570,571,575,589,614$ $617,619,649,652,654,666$ $668,679,682,727,729,737$ $743,744,768,788,805$

Hierro 110, 136, 140, 291, 298 $434,531,667,747,806$

Huelva 4, 20, 53, 113, 114, 129 , $155,176,213,238,248,251$ $268,298,299,303,336,372$ $386,397,398,399,402,409$ $417,440,442,461,476,485$ $492,523,527,532,545,565$ $569,574,584,587,590,619$ $633,637,644,649,650,652$ $662,666,674,679,682,700$ $705,706,723,737,756,761$ $773,785,800,802,803,808$

Huesca 4, 25, 32, 74, 129, 141 $154,155,156,157,176,181$ $191,199,213,221,229,236$ $248,249,250,257,260,261$, $262,267,273,274,276,277$ $282,298,302,303,308,309$, $316,319,323,336,360,365$, $372,373,386,389,390,392$, $394,396,399,400,402,406$ $409,418,426,427,430,431$, $432,435,459,461,467,468$ $485,492,493,495,507,514$ $516,523,525,527,532,545$ $546,553,561,562,565,569$, $570,571,587,589,592,614$ $617,628,631,633,635,637$ $638,648,649,650,652,654$ $662,671,679,682,699,704$ $709,723,727,728,729,735$ $737,740,741,749,760,766$ $768,779,789,806$

Ibiza $27,36,63,139,248,250$ $298,317,365,392,396,413$ $414,527,529,539,577,619$ $652,671,674,729$

Jaén 4, 14, 80, 113, 114, 129, 154, $155,157,159,213,219,221$ $230,236,238,240,248,261$, $263,267,273,281,282,298$ $304,308,309,316,319,323$ $345,358,365,372,389,390$ $396,399,401,402,412,419$ $427,430,434,461,464,484$ $485,491,492,495,507,508$, $523,524,527,532,545,555$ $565,569,570,571,575,581$ $584,587,590,615,617,633$ $637,638,648,649,652,655$ $662,665,666,679,681,682$ $699,703,706,715,717,723$ $725,726,727,729,735,737$ $741,747,749,779,802,806$

Lanzarote $110 ， 291 ， 298 ， 317$, 322,531

León 9, 62, 78, 80, 136, 154, 157 $159,189,196,199,213,214$ $236,238,240,257,263,268$ $273,282,298,303,308,316$ $319,323,336,365,372,373$ $389,390,400,409,426,430$ $453,461,485,492,493,495$, $507,514,516,523,527,569$ $570,571,614,617,633,637$ $638,649,650,654,662,666$ $679,682,699,723,724,727$ $729,737,738,739,745,756$ $768,779,781,796,798,802$

Lleida 4, 9, 27, 29, 74, 89, 129 , $154,155,156,157,173,176$, $185,195,199,213,217,218$, $236,240,248,249,258,261$, $263,273,274,281,282,298$, $303,308,309,316,319,360$, $363,366,372,386,390,394$, 396, 399, 400, 401, 402, 406, $409,418,427,430,433,435$, 453, 454, 459, 461, 468, 482, $484,485,492,493,507,523$ $527,530,532,534,545,546$, $553,555,561,562,564,565$, $568,569,570,571,575,577$, 589, 633, 637, 649, 654, 662, 671, 674, 679, 682, 699, 701, 703, 704, 723, 727, 729, 735, 737, 751, 753, 756, 765, 766, $768,785,796$

Lugo 129, 154, 213, 236, 248 , $260,263,267,274,298,336$, $372,390,397,400,453,461$, $473,485,495,507,514,523$, $527,553,569,631,633,649$, $650,652,654,662,679,682$, 723, 724, 727, 729, 737, 768, $785,788,796$

Madeira 87, 110, 159, 162, 220 , 236, 280, 291, 298, 307, 337, $398,399,456,461,474,484$ $488,532,535,555,565,568$, 576, 614, 657, 667, 674, 675, 714, 723, 729, 747, 750, 756, $803,808,815$

Madrid 2, 4, 5, 8, 9, 12, 14, 16, 19 $36,61,68,74,76,78,89,93$, $102,111,112,114,124,129$, $134,135,136,137,141,154$, $155,157,158,159,174,175$, $176,184,187,189,190,191$, 194, 196, 197, 199, 201, 204, 208, 209, 213, 216, 218, 225, $232,236,238,240,248,249$, $250,251,257,258,262,263$, $267,268,273,274,276,277$, $282,298,299,302,303,309$, $314,316,318,319,323,334$, $335,336,337,341,343,345$, $363,365,372,384,385,386$, $389,390,392,394,396,397$, $398,399,400,401,402,403$, $406,409,411,414,416,418$, $419,423,424,427,430,431$, $437,440,446,448,453,454$, $461,467,468,471,474,475$, $476,485,488,492,493,495$, $499,507,508,511,513,514$ $515,518,523,524,527,534$ $545,546,551,553,555,557$, 561, 563, 565, 566, 567, 569, $570,571,572,575,576,577$, $581,582,583,584,587,588$, $589,590,601,609,615,617$, 
191, 196, 202, 206, 213, 219 , $221,236,238,240,248,249$ $258,260,276,277,298,299$ $303,308,316,319,320,336$ $340,345,365,372,389,396$ $397,398,399,402,435,439$, $446,461,467,468,471,485$. $492,495,496,499,507,508$ $515,518,523,527,545,546$ $551,552,555,561,565,569$, $570,571,572,584,587,609$ $615,619,633,637,638,644$ $646,648,649,650,652,662$ $665,666,679,681,682,706$ $710,722,723,725,727,729$ $730,737,761,768,779,788$ $801,802,806,807,808$

Mallorca 3, 7, 25, 35, 36, 47, 63, $67,83,92,103,114,118,120$ $121,125,136,139,154,174$ $184,208,250,251,260,264$ $267,268,277,298,306,308$ $336,360,365,392,396,397$ $399,402,413,414,419,439$, $440,469,484,495,499,513$ $527,536,551,555,570,572$ $574,589,590,609,617,619$ $633,637,638,649,650,652$ $654,666,671,674,679,704$ $723,729,753,768,779$

Medas 341, 522

Melilla 28, 138, 159, 218, 221 $230,240,260,277,279,304$ $336,340,341,345,363,374$ $397,399,469,495,555,619$ 633,704

Menorca 4, 114, 174, 248, 263 $302,306,396,399,413,414$ $495,527,536,570,633,649$, $650,654,662,666,671,674$ $679,699,709,723,729,815$

Minho 280, 346, 365, 426, 436 $453,461,493,495,649,662$ $679,682,709,723,727,729$ $737,775,776,785,788,796$

Murcia 3, 4, 9, 22, 36, 113, 114 $129,143,154,155,157,159$ $172,174,176,187,191,193$ $201,213,222,232,235,236$ $240,248,249,257,259,261$ 262, 267, 273, 298, 308, 316, $319,322,336,341,345,365$, $372,386,387,389,392,395$ $396,398,399,400,406,409$, $412,426,427,428,430,440$ $461,468,485,492,495,497$ $507,513,523,527,545,551$, $555,561,565,569,570,571$ $575,587,589,590,633,637$ $649,650,652,654,662,666$ $674,679,681,682,686,693$ $697,706,723,737,768,771$ $779,801,802$

Navarra 3, 9, 53, 62, 69, 129 137, 154, 176, 196, 213, 236 $248,262,267,268,273,274$ $282,308,309,319,336,360$ $372,377,386,399,402,409$ $418,426,427,430,437,446$ $453,461,485,492,495,507$, $508,513,514,523,527,545$ $553,565,575,586,589,590$ $592,614,620,633,648,649$ $650,654,662,679,682,722$ $723,727,729,737,740,741$ $744,768,786,788$
Orense 4, 9, 37, 154, 155, 156 $176,191,196,201,213,236$ $248,249,250,257,258,263$, $273,274,277,298,299,303$ $336,372,392,397,400,430$ $453,461,485,495,507,508$ $523,527,553,565,587,633$ $636,649,650,652,654,662$, $679,682,709,723,737,768$ 785,788

Palencia 9, 80, 129, 154, 159 $160,172,181,189,208,213$ $217,236,240,273,281,319$ $337,365,394,485,492,493$, $507,514,523,527,565,570$, $587,617,637,649,652,662$ $679,682,699,737,760,768$, 796

Palma 4, 110, 128, 140, 162, 291 $298,303,434,531,667,747$ 806

Pontevedra 4, 30, 154, 155, 196 208, 213, 236, 248, 257, 258, $267,273,298,303,319,336$ $365,372,396,397,419,427$ $440,461,485,507,508,523$ $527,546,555,563,570,571$ $575,589,633,636,637,649$ $650,652,654,662,666,679$ $682,709,723,727,729,737$ $786,788,800,802$

Portugal (sin especificar) 9, 12 $53,62,114,119,137,155,159$. $172,181,184,191,200,201$ $213,214,224,230,236,237$ $248,257,267,276,280,298$, $306,308,316,322,323,338$ $344,345,346,385,389,390$, $391,396,399,411,413,415$, $418,437,440,448,461,467$, $489,494,496,507,508,512$ $513,514,527,533,534,555$ $563,571,575,581,584,614$, $617,619,630,636,644,649$ $656,662,669,673,674,680$, $681,682,686,688,689,693$ 699, 704, 705, 706, 722, 723 $727,733,737,756,768,775$ $786,796,806,815$

Ribatejo $347,365,402,461,679$ $723,729,775,776$

Rioja $129,137,154,176,191$ 196, 199, 208, 213, 236, 248 , $249,257,263,267,268,281$, $282,298,302,303,308,316$ $319,336,365,371,372,386$ $400,418,430,433,461,492$ $495,507,514,523,527,545$ $551,553,555,569,570,571$ $572,575,589,614,615,637$, $638,649,652,654,662,679$ $682,703,723,727,729,737$ $768,788,795$

Salamanca $4,9,30,78,91,129$ $154,155,159,176,189,196$ $199,208,213,236,249,260$ $268,273,298,323,343,345$, $365,372,393,396,397,399$, $402,419,430,461,468,485$, $492,493,495,507,514,523$ $527,545,561,569,570,584$ $608,616,621,636,637,638$ $649,651,652,653,661,662$, $671,674,679,682,684,704$ $709,713,722,723,727,737$ $753,769,779$
Segovia 4, 9, 12, 19, 20, 68, 74 $89,102,111,112,129,135$ $141,154,155,158,176,184$ $196,204,209,213,221,225$, $228,236,248,249,257,259$, $262,263,267,273,274,277$ $282,298,302,303,316,319$ $323,336,337,343,345,365$, $372,374,384,386,390,392$, $397,406,407,418,419,424$ $430,437,440,453,461,467$ $468,485,492,493,495,507$ $523,527,533,535,545,546$, $551,555,561,569,570,571$, $575,576,586,589,590,609$ $614,615,617,633,635,636$ $637,638,639,649,650,652$, $654,662,666,673,679,682$, $687,688,694,704,709,720$ $723,727,729,735,737,768$

Sevilla $4,14,36,97,113,129$ $143,154,157,162,172,174$, $205,213,214,218,235,236$, $248,249,250,259,267,268$ $298,302,303,316,336,365$, $386,389,392,396,397,398$, $399,400,409,427,430,440$, $446,462,467,468,475,485$ $492,495,499,507,514,515$ $518,523,527,545,552,555$ $561,565,569,570,571,572$, $575,577,584,587,614,617$, $623,633,637,649,650,652$. $654,655,662,665,666,668$, $674,679,681,682,690,698$ $700,709,723,725,729,737$, 779,806

Soria $154,159,184,191,196$, $199,213,236,248,257,259$ $261,268,273,308,316,373$, 386, 394, 399, 409, 418, 431, 433, 461, 468, 492, 495, 507, $513,514,523,527,553,569$ $571,586,590,637,649,650$ $652,662,679,682,727,729$, $737,768,788$

Tarragona $3,4,9,22,25,27,32$, $36,80,87,129,154,155,156$ $172,185,202,213,217,218$ $228,235,236,248,257,258$, 261, 264, 267, 298, 302, 303, $308,309,316,319,322,323$ $325,341,360,362,363,365$ $373,386,392,398,418,430$, $461,468,484,485,492,495$ $507,523,527,532,538,545$ $551,568,570,571,572,587$ $589,609,615,617,619,633$, $635,636,637,638,644,648$ $649,650,652,654,662,673$ $674,679,682,701,706,709$ $722,723,725,727,729,737$, $753,756,768,801$

Tenerife 4, 72, 78, 97, 107, 109, $110,116,120,128,136,140$, $162,177,218,223,262,291$, $298,307,314,317,340,342$ $364,406,427,434,483,485$, $496,500,522,531,555,564$. $575,667,747,736,806$

Teruel $37,127,129,135,154$ $155,156,157,159,176,189$ $191,196,209,213,236,238$, $240,248,249,257,259,260$, $263,264,267,273,276,277$ 281, 282, 298, 303, 304, 309.

$316,319,325,336,337,343$, $365,372,373,386,388,389$, 392, 394, 399, 401, 402, 408, $409,418,426,430,433,439$, $440,453,454,459,461,468$, $475,484,485,492,495,507$. $508,513,514,523,527,545$, $546,551,561,569,570,571$, 589, 590, 617, 631, 637, 644, $648,649,650,652,654,662$, $668,671,674,679,682,704$, 712, 722, 723, 727, 729, 737, $765,802,806,814$

Toledo 9, 20, 36, 129, 157, 159, 174, 208, 213, 232, 248, 251, 257, 258, 261, 268, 282, 298, 302, 316, 319, 323, 336, 365, $372,386,396,397,399,409$, 430, 442, 446, 461, 468, 475, $485,492,493,495,507,508$, 513, 514, 524, 527, 534, 545, $553,555,565,569,570,571$, 587, 589, 591, 601, 617, 629, 633, 636, 637, 638, 649, 650, $652,654,662,666,673,674$, $675,679,682,694,704,722$, 731, 737, 757, 769, 771, 779, 782

Tras-os-Montes $199,218,280$ $363,365,375,453,461,495$, 570, 649, 673, 679, 722, 723, 727, 729, 737, 768, 775, 776, 788

Valencia $3,4,9,22,28,33,36$, $38,78,127,129,136,154$, 155, 172, 176, 184, 191, 193, 199, 200, 204, 208, 213, 214, $221,235,236,238,240,248$, $249,250,251,257,258,259$, 260, 261, 262, 263, 267, 276, 277, 298, 299, 302, 308, 309, $319,322,336,337,341,343$, $345,360,365,367,372,373$, $389,392,396,398,399,400$, 401, 402, 409, 411, 426, 430, 446, 461, 468, 469, 471, 484, 485, 492, 495, 507, 508, 513, $514,518,523,527,546,551$, $555,557,561,564,565,569$, 570, 571, 572, 587, 590, 609, $617,619,631,633,637,638$, $647,649,650,652,654,662$, $665,666,668,670,671,673$ $674,679,682,683,685,688$, 699, 706, 723, 727, 729, 737, $768,771,802$

Valladolid $9,24,32,36,129$, 154, 155, 159, 209, 213, 236, 248, 257, 268, 298, 319, 336, $400,409,419,430,433,440$, 468, 492, 514, 523, 527, 545, $555,561,569,570,617,637$ 
Zamora 4, 9, 80, 154, 189, 199, Zaragoza 32, 61, 127, 129, 154, $213,260,262,273,298,365, \quad 155,157,158,159,171,174$ $400,461,467,485,492,493, \quad 176,196,213,218,236,238$, $507,514,523,545,555,565, \quad 248,249,259,262,263,267$, $570,571,587,621,638,649, \quad 268,273,274,276,298,303$ $745,768,779,788,796$
$390,392,396,399,400,402$ $419,426,427,431,439,440$ $461,468,485,492,495,507$ $513,514,523,527,545,546$ $549,553,555,561,562,569$ $570,571,572,575,577,588$ $589,590,592,614,617,633$,
$635,637,638,649,650,652$ $654,662,668,679,682,688$ $701,704,722,723,727,729$ $735,737,741,768,788,799$, 806 


\section{ÍNDICE DE PAÍSES E ISLAS}

Este índice contiene únicamente información de índole faunística, aunque sea mínima, y no se incluyen las menciones geográficas realizadas por otros motivos en la revista. Se relacionan por separado los países del mundo que hayan sido citados como tales, así como las islas de tamaño grande y los archipiélagos (éstos únicamente en los casos en que no se mencionan islas concretas). Salvo algunas excepciones, las citas de lugares sin indicación original del país al que pertenecen, así como las unidades geográficas compartidas entre varios países (ej. Cáucaso, Alpes), o los países comprendidos en indicaciones tipo "desde ... hasta ..." (ej. "desde Francia hasta Siberia"), no se incluyen. Tampoco se han indexado los casos en que las áreas de distribución se indican únicamente mediante líneas en mapas generales. Por Gran Bretaña e Irlanda se entiende las respectivas islas, por lo que no se utiliza la denominación política de Reino Unido (que incluye Irlanda del Norte). Los casos indexados como Checoslovaquia, Palestina, URSS y Yugoslavia, se refieren a indicaciones que no permiten averiguar fácilmente su ubicación en las naciones actuales (ej. por homonimia), en otros casos se ha tratado de indexar en su contexto político actual.

Afganistán 186, 264, 298, 398, $399,406,493,565,573,574$ $577,608,654,686,714,733$ 756,806

Albania 159, 238, 307, 366, 398 $461,495,683,722,729,756$

Alemania 9, 24, 42, 69, 78, 119, $141,164,197,213,258,262$, $274,298,306,324,336,337$ $343,346,347,365,366,390$ $399,406,417,419,427,435$ $437,453,461,467,474,482$ $499,514,532,533,535,546$ $551,565,569,571,572,573$ $576,577,583,584,587,588$ $630,639,648,656,671,689$ $699,722,723,727,743,745$ $747,751,753,756,765,768$ $769,785,797,816$

Andamán 398

Angola 239, 398, 619

Arabia Saudí 207, 264, 298, 299 $398,467,468,573,588,619$ 733, 747, 806

Argelia 7, 36, 80, 114, 120, 138, $139,155,164,174,179,184$ $201,213,230,235,236,258$ 260, 264, 268, 274, 276, 277 $278,279,280,281,298,304$ $306,307,322,323,324,336$, $346,365,372,385,391,392$ $396,399,414,416,418,419$, $428,430,456,467,468,469$ 484, 492, 495, 496, 497, 498 $513,529,530,551,569,573$ $577,584,588,601,608,617$ $619,637,648,652,654,656$ $667,674,689,699,701,704$ $708,722,723,725,729,733$ $736,737,756,801,802,806$

Argentina $115,119,235,343$ $461,488,502,535,564,608$ $708,714,732,747,752,754$ $755,756,777,787,805,809$

Armenia 135, 213, 298, 306, 426 Australia 39, 78, 119, 174, 213 232, 298, 299, 306, 398, 427 $450,457,488,498,499,538$ $551,558,564,572,573,587$ $588,644,652,656,674,675$, $714,723,732,747,748,751$ 757,813

Austria 164, 204, 213, 235, 236 , $269,273,298,306,324,343$ $366,372,399,406,419,427$ $430,433,456,461,467,484$ $488,532,546,551,565,567$ $572,577,587,617,633,649$,
$671,673,689,722,723,725$ $727,729,745,753,756,779$ $784,785,797,806$

Bahrein 398

Bélgica 9, 24, 42, 121, 213, 283 , $324,365,366,399,419,435$, $437,461,532,546,565,577$ $584,587,589,619,630,662$,
$703,722,745,753,776,785$, 797

\section{Belize 777}

Benín 467

Bismark 461

Bolivia 541, 714, 777, 787, 809

Borneo 416, 551, 748

Bosnia-Herzegovina 306, 390

Brasil 137, 197, 416, 480, 502 551, 619, 714, 723, 732, 736, 747, 777, 807

Brunei 748

Bulgaria 200, 347, 366, 375, 392 $419,437,488,496,516,532$,
$573,575,673,720,722,729$, $733,753,756,797,806$

\section{Buru 772}

Bután 398

Cabo Verde 110, 291, 412, 467 $522,573,614,619,750,803$

Camboya 398

\section{Camerún 747}

Canadá $9,78,306,336,346$ $375,419,477,499,514,532$, $538,546,558,565,571,573$ $576,585,587,619,722,733$, $747,756,765$

Carolinas 398, 564

Ceram 748

Cerdeña 7, 36, 117, 119, 120, 154 $159,172,174,298,306,307$ $324,363,365,373,391,419$ $426,437,456,469,551,667$, $701,722,723,725,753,815$

Chad 419, 587, 723

Checa, R. 24, 298, 366, 461, 532, 784, 797

Checoslovaquia $251,324,365$, $375,419,433,437,461,474$ $565,577,656,670,722,737$ $745,747,753,756$

Chile 13, 87, 499, 589, 747

China $80,153,213,236,283$ $298,306,336,392,398,406$ $440,453,488,493,516,551$, $552,572,654,656,714,722$, $733,736,747,748,756$

\section{Chios 385}

Chipre 220, 262, 264, 298, 365, $392,440,577,651,704,723,729$
Christmas 398

Cocos 398

Colombia 541, 747, 777

Comores 467, 733, 747

Congo 163,587

Córcega 7, 36, 117, 120, 154 $159,172,213,250,258,276$, $298,306,307,322,363,365$, $391,399,426,440,456,461$, $469,529,534,551,656,670$, $674,722,723,725,736,737$ $751,768,815$

Corea 213, 392, 493, 514, 572, $608,733,737,797$

Corfú 307, 391, 426

Costa de Marfil 239, 467, 564

Costa Rica 461, 488, 714, 747 . 759, 777, 787

Creta 36, 264, 298, 614, 619, $651,689,722,729$

Croacia 120, 213, 241, 250, 263 264, 274, 298, 306, 307, 398, 399, 401, 459, 516, 561, 584, $671,674,723,797$

Cuba 232, 634, 670, 733, 742, 777

Curaçao 578, 622

Dinamarca 24, 213, 262, 324 $365,417,419,427,461,565$ $576,577,587,589,619,639$, $649,723,734,737,745,747$ 768,797

Dominicana 232

Ecuador 714, 777, 809

EE.UU. de América 9, 71, 78 87, 137, 141, 207, 213, 232, $235,240,264,269,275,281$, $306,307,343,346,375,416$, $419,435,461,477,488,498$ $499,514,518,532,538,544$, $546,551,558,564,565,571$ 573, 576, 578, 587, 588, 589, $608,619,622,649,662,699$ 714, 722, 733, 736, 747, 751, $756,759,765,771$

Egipto 36, 80, 159, 164, 213, 240, 264, 278, 281, 298, 304, $307,365,396,399,469,474$, $488,497,514,551,565,574$ $587,588,589,609,623,654$ $656,657,673,674,701,722$, $723,733,756,806$

EI Salvador 777

Eslovaquia 366, 461, 784, 797

Eslovenia 324, 576, 747

Estonia 473, 514, 722

Etiopia 236, 298, 398, 467, 556, $573,582,679,736,747$
Feroes 619

Fidji 298, 398, 551, 656

Filipinas 126, 298, 398, 416, 564, $619,736,748$

Finlandia 213, 224, 306, 307, 316, $337,392,419,426,427,440$, $461,473,474,488,532,565$, 577, 587, 589, 674, 722, 737, $753,756,785,786,797,802$

Francia 2, 9, 20, 24, 78, 87, 117, $119,120,121,138,143,157$ $158,164,171,173,179,182$, 184, 186, 189, 191, 196, 204, 209, 213, 218, 224, 228, 232, $234,235,236,238,250,251$, 258, 261, 268, 269, 273, 276, 277, 283, 298, 299, 306, 307 , $308,316,322,323,324,325$, $341,346,360,363,365,366$, $372,374,375,377,385,386$, $389,390,391,392,396,398$, $399,403,406,409,412,413$, 416, 417, 418, 419, 427, 430, $435,437,439,440,453,454$, $456,457,459,461,467,468$, $473,474,481,482,484,489$ 492, 495, 497, 498, 499, 507, 511, 515, 516, 524, 527, 529, 532, 533, 534, 535, 538, 539, $545,546,551,552,559,563$, $564,565,568,570,571,572$, 575, 576, 577, 581, 584, 587, 588, 589, 592, 600, 608, 617, $619,630,637,638,639,648$, $649,652,656,662,663,667$. 669, 670, 673, 674, 679, 682, 683, 689, 699, 701, 703, 704, 708, 709, 720, 722, 723, 725, 727, 728, 729, 733, 735, 737, $740,745,747,751,753,756$, 766, 768, 769, 771, 775, 776, 779, 784, 785, 789, 796, 801, $802,803,807$

Gabón 163, 239

Galápagos 588

Gambia 564

Georgia 723

Gilbert 398, 564

Gran Bretaña 9, 20, 24, 42, 137, 141, 156, 164, 193, 196, 213, $220,231,232,235,240,298$, $306,307,315,324,325,337$, $346,355,365,375,391,399$, $400,410,417,419,424,427$, 430, 448, 461, 467, 473, 499, 507, 514, 519, 532, 534, 546, $551,555,558,565,577,583$, $584,587,588,589,592,614$, 
$619,643,648,649,654,656$, $662,699,703,704,722,723$ $727,733,743,745,747,753$ $756,757,765,768,769,785$ $797,803,807$

Grecia 120, 124, 154, 164, 224 $236,241,264,298,306,307$ $324,375,385,392,396,398$, $399,418,419,435,440,456$ $464,472,489,497,518,575$, $577,584,637,639,647,654$ $673,683,686,689,705,722$ $723,729,756,760,769,797$ 806

Groenlandia 419, 461, 532, 538, 619

Guatemala 714, 747

Guinea, R. 163

Guinea-Bissau 172

Guinea Ecuatorial 4, 119, 151, $163,180,239,262,290,343$ $416,484,575,806$

Guyana Francesa 777

Haití 777

Hawai 298, 419, 551, 564, 656, 747

Honduras 777

Hungría 119, 189, 208, 262, 298 , $302,306,324,343,365,366$, $399,419,427,440,461,474$ $484,496,546,565,572,577$ $587,601,639,673,689,722$ $723,727,729,733,747,753$ 756,797

India $232,236,240,264,298$ 299, 396, 398, 422, 477, 513, $538,546,551,555,564,571$, $572,573,574,582,587,588$, $619,649,656,677,688,714$ $720,722,723,733,736,747$ $748,760,797,806$

Indonesia 398

Irak 164, 264, 298，398，426, $493,514,563,574,587,689$

Irán 159, 200,201，218，236, $262,264,298,398,399,406$ 426, 497, 569, 574, 582, 686, $722,733,756,806$

Irlanda $137,307,337,419,426$, $461,558,565,575,577,614$ $619,643,648,674,699,704$ $722,727,785$

Islandia 419, 488, 546, 565, 588, 619,674

Israel 240, 298, 385, 398, 399 $419,514,515,565,573,587$, $588,619,656,657,683,689$ $704,722,723,745,747,753$ $756,806,813$

Italia $7,24,36,117,119,120$ $127,159,171,186,189,191$ $193,204,213,217,220,224$ $232,235,236,238,241,251$ $258,262,264,269,280,283$ 298, 306, 307, 316, 323, 324, $343,346,363,365,366,372$, $373,375,385,391,392,396$, $398,399,416,418,419,427$ $437,448,461,469,484,488$ 489, 495, 496, 497, 498, 516, $532,533,534,538,546,551$ $555,559,565,575,576,582$, $587,588,589,600,608,617$ $619,630,637,639,643,647$, $648,649,656,662,667,671$ $673,674,679,683,689,691$,
$699,704,708,709,720,722$ $723,725,727,733,737,745$ $747,751,753,756,768,769$ $779,785,786,794,797,801$ $802,803,806,815$

Jamaica 235, 551, 777

Japón 213, 217, 236, 283, 298, 299 $398,409,419,493,496,499$ $514,532,551,558,564,565$, $582,608,619,649,656,708$ $722,723,733,736,745,747$ $748,756,772,779,797,813$

Java $217,298,398,532,551$ $656,722,748$

Jordania $385,398,574,756$

Kazajistán 577, 722, 733

Kenia 299, 573, 656, 736, 747, 806

Kirguizistán 723

Kuriles 419, 797

Laos 398

Letonia 298, 365, 419, 473, 514 587

Líbano 213, 236, 298, 398, 406 $474,488,574,806$

Libia 36, 80, 159, 164, 264, 298, $300,307,398,469,472,559$, 574,729

Lituania 419, 514

Lofoten 619

Macedonia 614

Madagascar 76, 171, 174, 298 299, 336, 398, 467, 538, 551, $564,574,592,647,733,736$ 748

Malasia 398, 551, 564, 688, 747 748

Maldivas 398

Malta 264, 306, 307, 399, 534 $564,747,81$

Marianas 398, 564

Marquesas 398

Marruecos 3, 4, 28, 36, 48, 53, $80,110,114,119,120,138$, $139,140,146,155,156,157$ $159,160,174,191,200,201$, $213,218,221,230,240,259$, $260,261,268,275,276,277$ $278,279,280,281,291,298$ $299,304,305,306,307,323$, $324,325,339,341,342,343$, $345,346,347,363,365,373$, $374,391,392,396,397,399$, $406,411,413,414,416,419$ $427,435,437,438,440,442$ $456,459,461,467,469,474$ $483,484,488,489,492,495$ $496,497,498,499,513,514$ $515,522,551,552,555,561$ $563,569,572,574,575,577$, $589,608,614,619,630,648$ $667,674,686,689,699,700$ $704,712,722,723,725,727$ $729,733,737,745,760,761$ 768, 785, 789, 797, 800, 801, $802,806,811$

Marshall 398, 564

Mascareñas 748

Mauricio 280, 299, 723, 736, 747

Mauritania 300, 574, 619, 723

México 87, 203, 502, 538, 551, $588,634,691,714,722,723$ $732,747,777,787$

Midway 398, 419

Molucas 748

Mónaco 461
Mongolia 159, 213, 298, 396, $419,437,608,614,654,662$ $722,727,733,756,806$

Mozambique 299, 398, 589, 619 679

Myanmar (Birmania) 298, 398 , 722,765

Namibia 574, 806

Nepal 236, 398, 733, 747

Nicaragua 299, 777

Nicobar 398

Nigeria 484, 534, 686, 714, 806

Norfolk 398

Noruega 224, 532, 565, 577, 619, $674,737,747,753$

Nueva Caledonia 398, 538, 564 , 770,772

Nueva Guinea 398, 506, 564 $733,736,747,748,772$

Nueva Zelanda $306,324,419$ $474,488,498,506,535,558$ $564,619,649,674,675,708$ $751,756,757,772,813$

Nueva Zembla 461

Nuevas Hébridas 398, 748

Okinawa 748

Omán 806

Paises Bajos 9, 42, 78, 196, 211 $324,343,365,366,419,430$, $488,516,532,546,551,587$ $589,619,639,662,722,723$ $745,769,771,776,785,786$ 797

Pakistán 264, 298, 398, 574, 720 733, 747, 806

Palestina 80, 213, 391, 399, 440, 722

Panamá 240, 588, 777

Paraguay 714,777

Perú 714, 732, 747, 757, 777, 809

Phoenix 398

Polonia 213, 306, 366, 419, 461, $474,532,565,571,573,588$ 708, 722, 723, 729, 737, 745, 797

Puerto Rico 235, 714

Reunión 723, 733, 747

Rodas 440, 493, 574, 686, 722

Ruanda 747

Rumania 216, 256, 343，366 417, 419, 427, 437, 497, 565, $567,572,587,639,673,722$ $727,745,747,797,806$

Rusia 135, 159, 172, 201, 204 $213,224,236,240,250,262$ $298,306,308,316,325,343$ $365,366,371,375,391,392$ $396,399,400,401,411,419$ $427,437,440,456,468,473$ $493,495,497,513,523,546$, $565,569,570,571,572,573$ 577, 582, 587, 588, 608, 614, $617,637,639,649,654,662$, $671,673,674,699,722,723$ $727,733,753,797,806$

Ryu Kyu 398, 736, 747,748

Sahara Occidental 100,138 $163,240,291,340,345,416$, $442,496,522,686,760$

Sajalin 213, 722

Salomón 398, 551, 564

Samoa 398, 564

Santa Helena 298

Santo Tomás 163

Sarawak 298

Senegal 163, 172, 240, 435, 467, $477,484,497,619$ Senegambia 619

Serbia-Montenegro 461, 722, 797

Seychelles 551, 723, 748

Sicilia $36,111,119,120,139$ $159,171,172,174,213,260$, 264, 268, 276, 278, 280, 298, $306,307,314,363,365,373$, $391,398,399,416,426,427$ $437,456,461,467,469,533$, 534, 536, 551, 555, 570, 577, $614,649,663,668,708,722$, $723,725,745,779,806,815$

Sierra Leona 163, 239, 435

Singapur 398,748

Siria 80, 159, 201, 213, 220, 236, $241,258,278,298,299,306$, $391,396,398,399,406,427$, $435,440,456,551,569,572$, $574,577,589,619,654,662$, $689,722,723,725,733,757$

Sociedad 398, 564

Somalia 172, 207, 299

Sonda 748

Spitzbergen (= Svalbard) 419, 538

Sri Lanka $164,217,298,398$, $427,467,477,480,551,564$, 619, 656, 679, 722, 723, 733, $736,747,748,806$

Sudáfrica 191，298，299，306, 398, 467, 477, 489, 499, 573, 574, 587, 619, 656, 714, 723, 731, 733, 736, 747, 757, 800, 806

Sudán 172, 207, 239, 299, 398, $551,573,588,747,806$

Suecia 213, 224, 236, 274, 298, $302,306,337,365,372,375$, 401, 417, 419, 427, 461, 495, $523,532,535,565,571,575$. $577,583,587,589,619,639$, 649, 673, 722, 723, 727, 737, $743,745,753,759,786$

Suiza 9, 204, 234, 251, 298, 324 $337,346,365,392,419,427$, $435,440,461,471,474,507$, $516,527,533,535,546,565$, $589,656,667,671,689,704$ $722,725,729,733,743,745$. $747,753,779,797$

Sulawesi (Célebes) 398, 551, 748

Sumatra 398, 497, 656, 736, 748, 772

Surinam 813

Tailandia 398, 748

Taiwan (Formosa) 213, 275, 298, 398, 532, 551, 565, 588, $649,736,747,748,797$

Tanzania $163,731,736,747,806$ 
$674,701,704,722,723,725$ $756,801,802,806$

Turkmenistán 264, 722, 799, 806

$729,733,745,747,756,769$ 785,806

Ucrania 366, 384, 392, 437, 461 $514,693,806$

Turquía 164, 213, 236, 298, 305, Uganda 163, 299, 551, 587, 588, $306,308,375,398,399,406, \quad 736,747,806$

460, 497, 555, 561, 565, 567, URSS 399, 551, 649, 656, 714 569, 574, 608, 619, 648, 667, $674,689,704,705,722,727$,
$723,747,756$

Uruguay $723,747,777$
Uzbekistán 407, 722, 723, 733

Venezuela 87, 541, 619, 747, 777

Vietnam 398, 748

Wallis 398

Yemen 207, 298, 686, 733, 779,$$
806
$$

Yugoslavia 143,164,184, 209 $224,235,236,298,306,310$, $324,366,385,399,406,427$
$461,474,516,567,587,588$ $630,673,686,722,723,727$, $29,753,756$

Zaire (R.P. Congo) 163, 239,

$298,551,564,733,736$

Zanzíbar 619

Zimbabue 299, 534, 588, 747, 806 
